\title{
D-Area Coal Pile Runoff Basin Groundwater Treatability Study Preliminary Report
}

by

F. A. Washburn

Westinghouse Savannah River Company

Savannah River Site

Aiken, South Carolina 29808

M. E. Denham

DEC 2,1909

M. A. Phifer

OST1

F. C. Sappington

DOE Contract No. DE-AC09-89SR18035

This paper was prepared in connection with work done under the above contract number with the U. S.

Department of Energy. By acceptance of this paper, the publisher and/or recipient acknowledges the U.S. Government's right to retain a nonexclusive, royalty-free license in and to any copyright covering this paper, along with the right to reproduce and to authorize others to reproduce all or part of the copyrighted paper. 


\title{
D-AREA COAL PILE RUNOFF BASIN GROUNDWATER
}

\author{
TREATABILITY STUDY PRELIMINARY REPORT (U)
}

\section{SEPTEMBER 1998}

\section{UNCLASSIFIED}

Does Not Contain Unclassified Nuclear Information (UCNI)

$\mathrm{ADC}$ and Reviewing Official

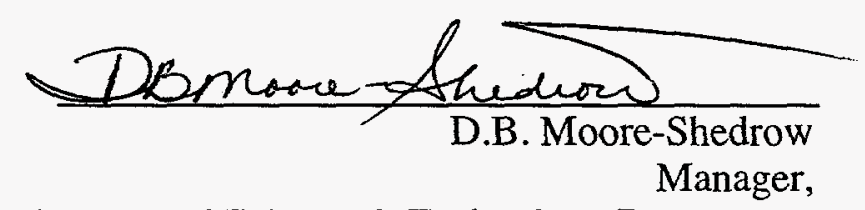

Environmental Science \& Technology Department

Westinghouse Savannah River Company

Savannah River Site

Aiken, SC 29801

Prepared for the US Department of Energy under Contract DE-AC09-89SR18035 


\section{DISCLAIMER}

This report was prepared as an account of work sponsored by an agency of the United States Government. Neither the United States Government nor any agency thereof, nor any of their employees, makes any warranty, express or implied, or assumes any legal liability or responsibility for the accuracy, completeness, or usefulness of any information, apparatus, product, or process disclosed, or represents that its use would not infringe privately owned rights. Reference herein to any specific commercial product, process, or service by trade name, trademark, manufacturer, or otherwise does not necessarily constitute or imply its endorsement, recommendation, or favoring by the United States Government or any agency thereof. The views and opinions of authors expressed herein do not necessarily state or reflect those of the United States Government or any agency thereof.

This report has been reproduced directly from the best available copy.

Available to DOE and DOE contractors from the Office of Scientific and Technical Information, P.O. Box 62, Oak Ridge, TN 37831; prices available from (615) 576-8401.

Available to the public from the National Technical Information Service, U.S. Department of Commerce, 5285 Port Royal Road, Springfield, VA 22161. 


\section{DISCLAIMER}

Portions of this document may be illegible in electronic image products. Images are produced from the best available original document. 


\section{D-AREA COAL PILE RUNOFF BASIN GROUNDWATER TREATABILITY STUDY WORK PLAN (U)}

Revision 0 Prepared By:

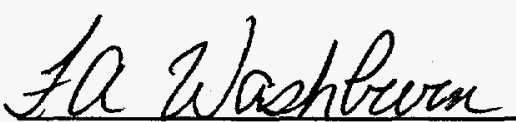

F.A. Washburn

Environmental Sciences and Technology Department Savannah River Technology Center

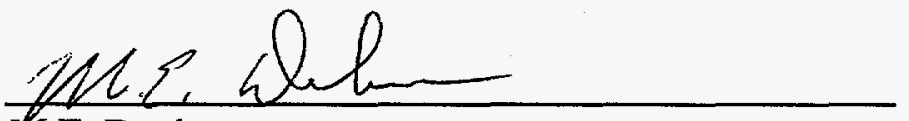

M.E. Denham

Environmental Sciences and Technology Department Savannah River Technology Center

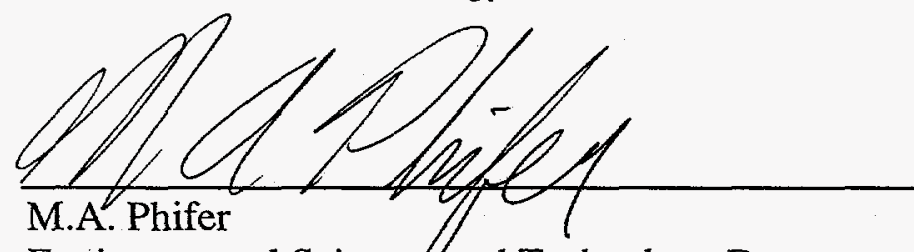
Environmental Sciences and Technology Department Savannah River Technology Center

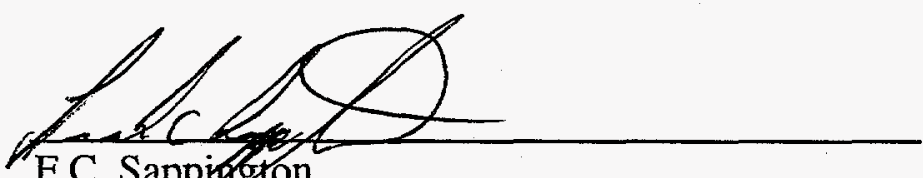

F.C. Sappirifton

Environmental Sciences and Technology Department Savannah River Technology Center

Reviewed and Approved By:

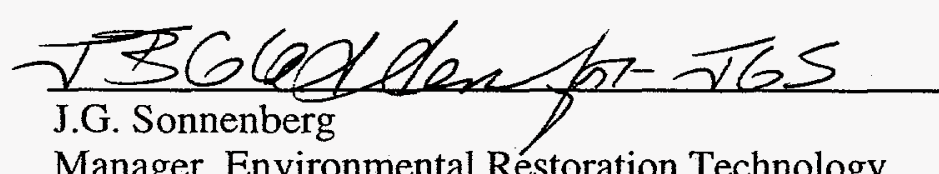

Manager, Environmental Restoration Technology Environmental Sciences and Technology Department Savannah River Technology Center 


\section{TABLE OF CONTENTS}

\section{LIST OF ACRONYMS}

\section{INTRODUCTION}

1.1. Site Description and History

1.2. Waste Characteristics

1.3. Project Description/DOE Needs

1.4. Groundwater Characterization

\section{TREATABILITY STUDY OBJECTIVES}

2.1. General

2.2. Functional Performance Requirements

2.2.1. Ex-Situ Treatment Media Evaluation

2.2.2. D-Area GeoSiphon Treatment System Conceptual Design

3.2. Technology/Process Description

2.1.3. Ex Situ Treatment Media Evaluation

3.2.2. Existing Barrier Wall System

3.2.3. GeoSiphon Treatment System Conceptual Design

4.1. Test Campaigns

4.1.2 Field Run \#2 (4/98)

4.1.3 Field Run \#3 (7/98-8/98)

4.1.4 Lab Test \#1 (5/98)

4.1.5 Lab Test \#2 (6/98)

4.1.6 Lab Test \#3 (7/98)

4.1.7 Lab Test \#4 (9/98) 


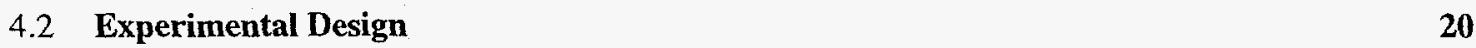

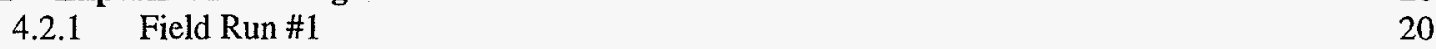

\begin{tabular}{lr}
4.2 .2 & Field Run \#2 \\
\hline & 21
\end{tabular}

$\begin{array}{ll}4.2 .3 \quad \text { Field Run \#3 } & 24\end{array}$

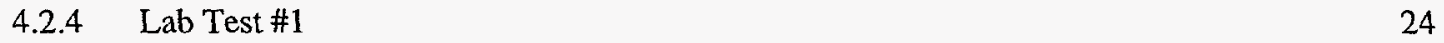

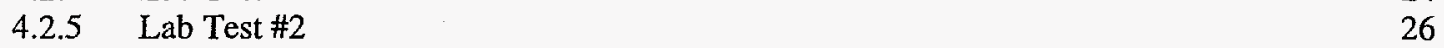

4.2.6 Lab Test \#3 . 27

$\begin{array}{lll}4.2 .7 & \text { Lab Test \#4 } & 28\end{array}$

4.3 Equipment, Materials \& Methods $\quad 29$

$\begin{array}{ll}4.3 .1 \quad \text { Field Run \#1 } & 29\end{array}$

\begin{tabular}{ll}
4.3 .2 & Field Run \#2 \\
\hline
\end{tabular}

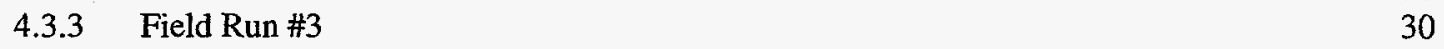

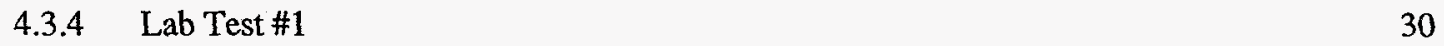

$\begin{array}{ll}\text { 4.3.5 Lab Test \#2 } & 31\end{array}$

$\begin{array}{lr}\text { 4.3.6 Lab Test \#3 } & 31\end{array}$

$\begin{array}{ll}\text { 4.3.7 Lab Rün \#4 } & 32\end{array}$

4.4 Sampling and Analysis $\quad 32$

$\begin{array}{llr}4.5 & 39\end{array}$

$\begin{array}{llr}4.6 & \text { Deviations } & 39\end{array}$

5. RESULTS AND DISCUSSION 39

$\begin{array}{lll}5.1 & \text { Field Run \#1 }\end{array}$

$\begin{array}{lll}\text { 5.1.1 Initial Design Concept Prior to Media Studies } & 39\end{array}$

5.1.2 Field Observations Field Run 1 41

5.1.3 Data Observations Field Run 1 43

$\begin{array}{llr}\mathbf{5 . 2} & \text { Field Run \#2 } & 59\end{array}$

5.2.1 Revised Design Concept following Field Run 1

$\begin{array}{lll}5.2 .2 & \text { Field Observations Field Run } 2 & 60\end{array}$

5.2.3 Data Observations Field Run 2 61

5.3 Field Run \#3

5.3.1 Conceptual Design $\quad 66$

5.3.2 Field Observations Field Run $3 \quad 67$

$\begin{array}{lll}\text { 5.3.3 Data Observations Field Run } 3 & 68\end{array}$

$\begin{array}{llr}5.4 & \text { Laboratory Test \#1 } & \mathbf{7 3}\end{array}$

$\begin{array}{lll}\text { 5.4.1 Laboratory Observations } & 73\end{array}$

$\begin{array}{lll}\text { 5.4.2 Data Observations Lab Test \#1 } & 74\end{array}$

$\begin{array}{llr}5.5 & \text { Laboratory Test \#2 } & \mathbf{8 1}\end{array}$

$\begin{array}{lll}\text { 5.5.1 Laboratory Observations } & 81\end{array}$

5.5.2 Data Observations Lab Test \#2 81

$\begin{array}{llr}5.6 & \text { Laboratory Test \#3 } & \mathbf{8 6}\end{array}$

5.6.1 Laboratory Observations $\quad 86$

5.6.2 Data Observations Lab Test \#3 87

$\begin{array}{lll}5.7 & \text { Laboratory Test \#4 } & 90\end{array}$ 
5.7.1 Laboratory Observations

90

5.7.2 Data Observations Lab Test \#4

5.8 System Chemistry

6. CONCLUSIONS, RECOMMENDATIONS, AND GEOSIPHON TREATMENT SYSTEM CONCEPTION DESIGN

7. KEY CONTACTS AND PARTICIPANTS

8. REFERENCES

9. APPENDICES 


\section{LIST OF ACRONYMS}

$\begin{array}{ll}\text { CERCLA } & \text { Comprehensive Environmental Response Compensation and Liability Act } \\ \text { CPRB } & \text { Coal Pile Runoff Basin } \\ \text { DOE } & \text { Department of Energy } \\ \text { Eh } & \text { Redox potential referenced to the standard hydrogen electrode } \\ \text { EM } & \text { Environmental Management } \\ \text { ERD } & \text { Environmental Restoration Department } \\ \text { FFA } & \text { Federal Facilities Agreement } \\ \text { FY } & \text { Fiscal Year } \\ \text { gPm } & \text { Gallons per minute } \\ \text { HDPE } & \text { High Density Polyethylene } \\ \text { HQ } & \text { Headquarters } \\ \text { MG } & \text { Million gallons } \\ \text { MGD } & \text { Million gallons per day } \\ \text { NPDES } & \text { National Pollutant Discharge Elimination System } \\ \text { OST } & \text { Office of Science and Technology } \\ \text { pH } & \left.\left.\text { pH (A measurement of groundwater acidity: -log[H }{ }^{+}\right]\right) \\ \text {RCRA } & \text { Resource Conservation and Recovery Act } \\ \text { S } & \text { Sulfur } \\ \text { SRS } & \text { Savannah River Site } \\ \text { TS } & \text { Treatability Study } \\ \text { TSR } & \text { Treatability Study Report } \\ \text { WSRC } & \text { Westinghouse Savannah River Company } \\ \end{array}$




\section{EXECUTIVE SUMMARY}

This document presents the Treatability Study Preliminary Report for the D-Area Coal Pile Runoff Basin (CPRB) Groundwater at the Savannah River Site. The groundwater at this facility is similar to acid mine drainage with low $\mathrm{pH}$ and elevated concentrations of metals. The groundwater is contaminated as a result of contaminants migrating from the bottom of the D-Area Coal Pile Runoff Basin to the groundwater. The D-Area CPRB is a permitted wastewater treatment facility with a National Pollutant Discharge Elimination System permitted discharge for the treatment of runoff from the D-Area coal pile. The basin was designed as a sedimentation/seepage basin for the removal of suspended solids and to minimize the direct discharge of coal pile runoff to Beaver Dam Creek.

The D-Area Coal Pile Runoff Basin groundwater is contaminated and requires response per the CERCLA process in accordance with the Federal Facility Agreement for the Savannah River Site. Another project at the D-Area CPRB was implemented during fiscal years 1994 through 1996 involving the design, installation, and testing of a barrier wall system within the most contaminated portion of the D-Area CPRB groundwater plume. The objective of this project is to demonstrate the utilization of the existing barrier wall system as part of a treatment system for the metals contaminated groundwater at the DArea Coal Pile Runoff Basin, and to evaluate various permeable treatment media or combinations of media for the treatment of this groundwater. The primary objective of the treatment system is to remove metals from the groundwater.

The utilization of the existing barrier wall system to produce a treatment system for the metals contaminated groundwater at the D-Area Coal Pile Runoff Basin will be accomplished in two phases: (1) Ex situ treatment media evaluation and (2) D-Area GeoSiphon Treatment System conceptual design.

The ex situ treatment media evaluation is a treatability study conducted at the D-Area Coal Pile Runoff Basin utilizing the actual contaminated groundwater. Treatment media evaluated included: limestone, rock phosphate (apatite), iron, peat, and zeolites and various phosphate compounds. The tests were conducted at the D-Area Coal Pile Runoff Basin using the existing barrier wall system (DIW-1, Interceptor Well) and surrounding monitoring wells to extract the contaminated groundwater for testing. The field treatment media was contained in in-line canisters, and influent and effluent samples were taken for chemical analysis to determine the effectiveness of each treatment media.

Seven campaigns were completed for this test:

- Field Run 1 - Contact Media Test

- Field Run 2 - Non Contact Media Test

- Field Run 3 - Reactive/Precipitative Contact Media Test

- Lab Test 1 - Non-Contact Soluble Phosphate Media

- Lab Test 2 - Non-Contact Soluble Phosphate Media (con't)

- Lab Test 3 - Particle Size and Contact Time Study

- Lab Test 4 - Aeration Study 
Based on initial conditions, a minor $\mathrm{pH}$ adjustment was sufficient to precipitate the chromium phases in our groundwater. Nickel, on the other hand, was more difficult to precipitate by $\mathrm{pH}$ adjustment alone. Even at high dissolved phosphate concentrations $(9500 \mathrm{mg} / \mathrm{L})$, nickel phosphates will only precipitate after a considerable increase in $\mathrm{pH}$. For this reason, it is assumed that nickel must co-precipitate with other metals. The presence of high concentrations of iron in the groundwater provides a potential mechanism for nickel removal by co-precipitation with iron phases.

As determined from Field Run \#1, removal of the contaminants of concern by precipitation in the first bulk precipitation canister and by cation exchange or further increases in $\mathrm{pH}$ in the second polishing canister. From the preliminary results of Field Run \#1, it appears as long as the bulk precipitation process is successful, the polishing process works rather well. Significant emphasis was placed on these campaigns to refine the bulk precipitation process. It should be noted that the overall treatment process should consider all potential combinations to provide the best possible treatment.

While results are preliminary, the following general overall conclusions for the entire operating regime are deduced:

- Both aluminum and chromium can be effectively treated with limestone and phosphate

- Iron can also be treated with limestone and phosphate.

- While iron is significantly removed, treatments are not as effective as those for aluminum and chromium.

- Treatments that enhance iron removal also enhance nickel removal

- Nickel is probably co-precipitated with iron.

The following should be considered for further evaluation:

- All data should be looked at in greater detail by statistical analysis, mass balance, etc.

- Comparison of data and treatment effectiveness should be performed

- In particular, ammonium phosphate should be evaluated more closely due to its performance in Run \#4.

These recommendations are being considered in FY99.

Based on the preliminary data results and field observations, a conceptual design which includes GeoSiphon cells is under evaluation. GeoSiphon Cells are passive groundwater treatment systems which utilize siphon flow between two points of natural head difference to induce greater than natural contaminated groundwater flow through a treatment train without the requirement for external energy input. The treatment train utilized in GeoSiphon Cells can include permeable treatment materials, aeration, chemical addition, etc. 
The conceptual GeoSiphon Cell configuration includes extraction of the contaminated water from the mound created by the barrier wall system by siphon to a downgradient treatment system which may consist of an oxidizing step such as aeration followed by a permeable treatment media or treatment media combination. The siphon flow will be induced by the natural hydraulic head difference between the mound upgradient of the wall and the adjacent D-Area discharge stream, which is a lower hydraulic head. Further development of this concept is anticipated in FY99.

Advantages of this conceptual design include:

- Utilization of the existing barrier wall system as part of a treatment system for the D-Area CPRB metals contaminated groundwater. The existing barrier wall system represents a significant past Office of Science and Technology (OST) investment.

- Utilize natural hydraulic driving forces to induce flow for remediation.

- Treatment within the most heavily contaminated portion of the D-Area CPRB plume.

- Removable treatment options so that heavy metals can be collected and recycled.

- Potentially accelerate clean up, can be applied to a broader range of site conditions, and can increase treatment media longevity and effectiveness.

- Minimize media armoring and/or cementation, and formation pluggage.

- Directly meeting the needs of the EM-40 program (Environmental Restoration).

All work will be conducted in conjunction with the SRS Environmental Restoration Department to assure that the work will meet the end users needs and conform to regulatory expectations. 


\section{INTRODUCTION}

\subsection{Site Description and History}

This document presents the Treatability Study Preliminary Report for the D-Area Coal Pile Runoff Basin (CPRB) Groundwater at the Savannah River Site (SRS). The D-Area Coal Pile Runoff Basin is a permitted wastewater treatment facility with a National Pollutant Discharge Elimination System (NPDES) permitted discharge (D-001B) for the treatment of runoff from the D-Area coal pile. The basin was designed as a sedimentation/seepage basin for the removal of suspended solids and to minimize the direct discharge of coal pile runoff to Beaver Dam Creek. The CPRB is also listed in the Federal Facility Agreement (FFA) for the Savannah River Site as a RCRA/CERCLA unit. Figure 1 shows the location of the basin.

The basin was built in 1978. It has an average bottom elevation of 121.0 feet, a top of berm elevation of 125.5 feet, an operating water level elevation of between 121.0 and 124.5 feet, valved discharge piping capable of draining the basin to 121.0 feet, and a concrete emergency spillway at an elevation of 124.84 feet. It has a surface area, operating volume, and maximum volume of 12.5 acres, 10 to 13 million gallons (MG), and 14.5 MG, respectively. The runoff from the D-Area coal pile contained in the basin is drained at an estimated 75 to 100 gallons per minute (gpm), through discharge piping to a natural discharge ditch that drains to Beaver Dam Creek, whenever the basin water elevation reaches approximately 124.5 feet. The discharge ditch has a long-term flow of 55.8 million gallons per day (MGD).

\subsection{Waste Characteristics}

The 484-D PowerHouse burns coal to produce electricity and steam for use at SRS. In general a 90 day supply of moderate to low sulfur coal $(1-2 \% \mathrm{~S})$ is stored in an open, 8.9 acre pile adjacent to the 484-D PowerHouse. The coal is not rotated which may result in long-term exposure of some coal to the environment. Long term chemical and biological oxidation of the sulfur compounds produces sulfuric acid. Rain water along with the sulfuric acid leach other impurities from the coal. This produces an acidic runoff with substantial metals contamination which is collected and discharged via gravity drainage to the CPRB. This runoff predominately seeps into the water table aquifer through the bottom of the CPRB. The D-Area Coal Pile Runoff Basin groundwater is contaminated with low pH, and elevated concentrations of iron, aluminum, sulfate, chromium, and nickel with lesser amounts of trichloroethylene, cadmium, and lead. 


\section{D-Area Coal Pile Runoff Basin Location Map}

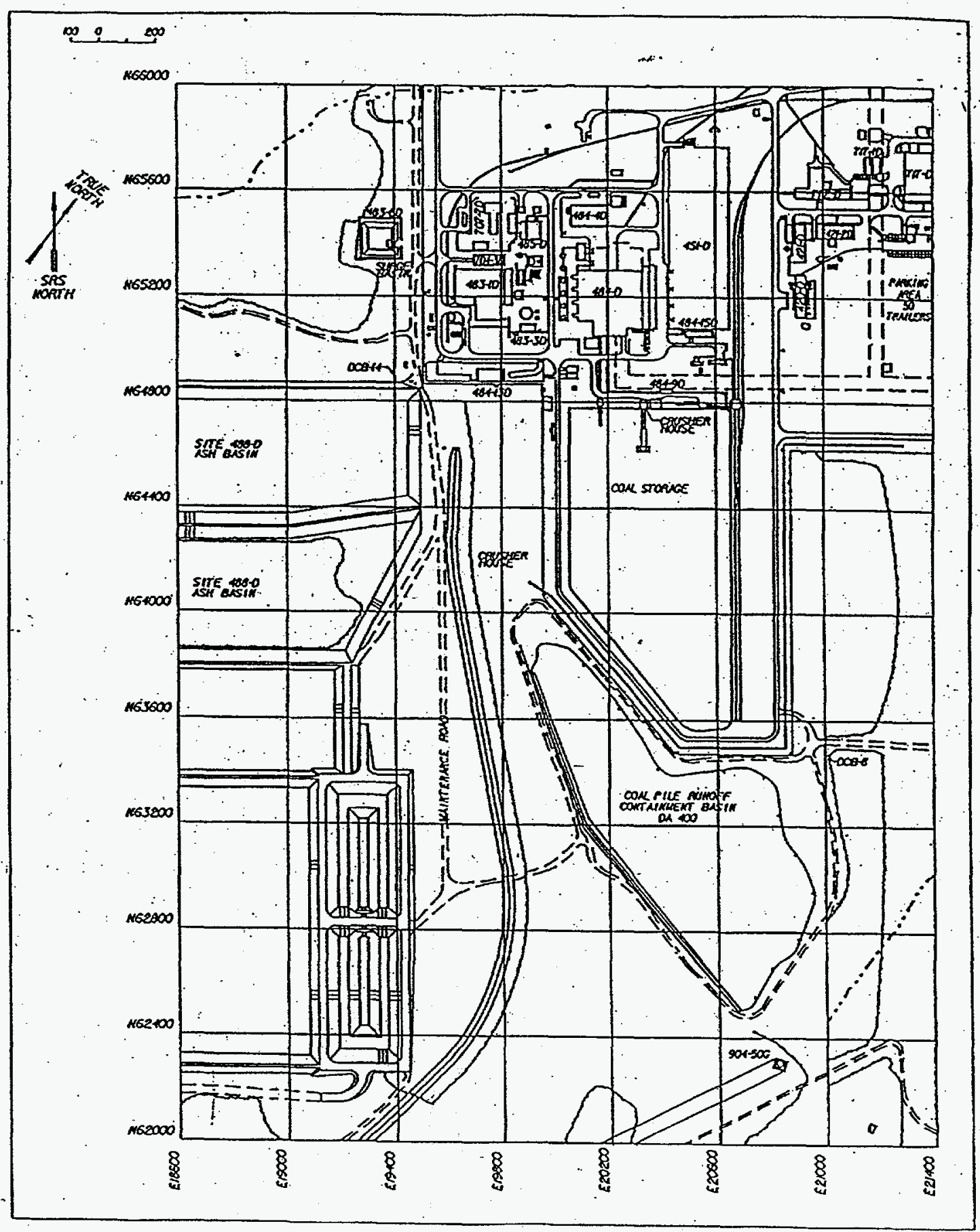

Figure 1 - Location of the D-Area Coal Pile Runoff Basin 


\subsection{Project Description/DOE Needs}

The D-Area Coal Pile Runoff Basin groundwater is contaminated and requires response per the CERCLA process in accordance with the Federal Facility Agreement for the Savannah River Site. Another project at the CPRB was implemented during fiscal years 1994 through 1996 involving the design, installation, and testing of a barrier wall system within the most contaminated portion of the D-Area CPRB groundwater plume. The objective of this project is to demonstrate the utilization of the existing barrier wall system as part of a treatment system for the metals contaminated groundwater at the CPRB, and to evaluate various permeable treatment media or combinations of media for the treatment of this groundwater. The primary objective of the treatment system is to remove metals from the groundwater.

The utilization of the existing barrier wall system to produce a treatment system for the metals contaminated groundwater at the Coal Pile Runoff Basin will be accomplished in two phases:

- Ex situ treatment media evaluation.

- D-Area GeoSiphon Treatment System conceptual design.

Further development of the GeoSiphon Treatment system is anticipated in FY99.

Four area programs have registered their need for a passive groundwater treatment system for metals and/or radionuclides:

- Innovative Technologies to Replace Pump and Treat Technologies for Groundwater Remediation (SR-3018)

- Availability of Innovative Treatment for Radionuclide and Metal Contamination in Groundwater (AL-97-09-02-SC)

- Remediation of Strontium-90 Contaminated Groundwater (CH0001)

- Reactive Barrier Treatment Systems (HY13)

\subsection{Groundwater Characterization}

Table 1 provides information on the influent, groundwater, and effluent water quality associated with the CPRB. The groundwater data is based upon maximum well results in the vicinity of the existing barrier wall system (wells DCB-1A, -10 , $-18 \mathrm{~A},-18 \mathrm{~B},-18 \mathrm{C},-19 \mathrm{~B},-19 \mathrm{C},-21 \mathrm{~B},-21 \mathrm{C},-22 \mathrm{~A},-22 \mathrm{~B}$, and $-22 \mathrm{C})$. This groundwater is very acidic ( $\mathrm{pH}$ is approximately 2.2 ) and is contaminated primarily with sulfate, iron, aluminum, chromium, and nickel as a result of impurities leached from the coal pile. 


\section{TREATABILITY STUDY OBJECTIVES}

\subsection{General}

The acceptance criteria associated with this technology includes the following:

- A suitable permeable treatment media or combination of media identified for the treatment of the D-Area CPRB metals contaminated groundwater.

- The removal of metals from the contaminated groundwater.

Table 1. D-Area Coal Pile Runoff Basin Water Quality

\begin{tabular}{|c|c|c|c|c|}
\hline Parameter & Influent & Groundwater $^{2}$ & Effluent $^{3}$ & $\begin{array}{l}\text { NPDES Outfall } \\
\text { D-001B Limits } \\
3\end{array}$ \\
\hline Aluminum, ppb & 398,000 & 301,000 & 40,200 & - \\
\hline Arsenic, ppb & 135 & LD & NA & - \\
\hline Barium, ppb & LD & 35.5 & NA & - \\
\hline Beryllium, ppb & NA & 177 & NA & - \\
\hline Cadmium, ppb & 74 & 11.8 & NA & - \\
\hline Chromium, ppb & 329 & 182 & NA & - \\
\hline Cobalt, ppb & NA & 1970 & NA & - \\
\hline Copper, ppb & 2,230 & 418 & NA & - \\
\hline Iron, $\mathrm{ppb}$ & $1.45 \times 10^{6}$ & $2.16 \times 10^{5}$ & 174,000 & - \\
\hline Lead, ppb & 2.5 & LD & NA & - \\
\hline Magnesium, ppb & 308,000 & 132,000 & 17,800 & - \\
\hline Manganese, ppb & 59,000 & 104,000 & 2,380 & - \\
\hline Mercury, ppb & $0.15(0.185)^{4}$ & $2.12^{5}$ & NA & - \\
\hline Nickel, ppb & 7,900 & 1,660 & NA & - \\
\hline Nitrate/Nitrite as $N, p p b$ & NA & 537 & NA & - \\
\hline $\mathrm{pH}$ & 2.26 & 2.2 & $6.6^{6}$ & $6-9^{6}$ \\
\hline Selenium, ppb & 6.3 & LD & NA & - \\
\hline Sulfate, ppb & $8.46 \times 10^{6}$ & $8.43 \times 10^{6}$ & $1.0 \times 10^{6}$ & - \\
\hline TCE, ppb & NA & 80.4 & NA & - \\
\hline Total Organic Carbon, ppb & NA & 4920 & NA & - \\
\hline Total Suspended Solids, ppb & 54,000 & NA & 1,700 & 50,000 \\
\hline Vanadium, ppb & NA & 59.1 & NA & - \\
\hline Zinc, ppb & 14,100 & 5,080 & 1,040 & - \\
\hline Gross Alpha ${ }^{7}, \mathrm{pCi} / \mathrm{L}$ & NA & 176 & NA & - \\
\hline Nonvolatile Beta ${ }^{7}, \mathrm{pCi} / \mathrm{L}$ & NA & 66.7 & NA & - \\
\hline Radium ${ }^{7}, \mathrm{pCi} / \mathrm{L}$ & NA & 2.91 & NA & - \\
\hline Tritium, pCi/mI & NA & 9.21 & NA & - \\
\hline \multicolumn{5}{|c|}{$\begin{array}{l}\text { Notes for Table 1: } \\
-N A=\text { NNot Analyzod } \\
\text { LD = less than yetection limit } \\
\text { ISRL-ESS-B9-745 (intemal October, } 1985 \text { compositc sample ressults). }\end{array}$} \\
\hline \multicolumn{5}{|c|}{ 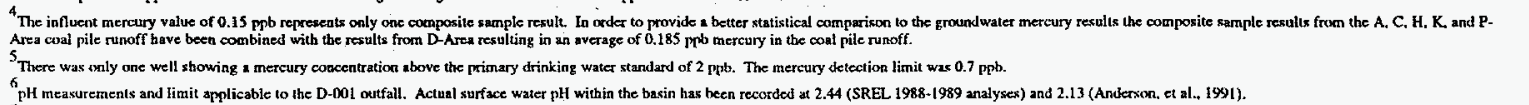 } \\
\hline
\end{tabular}




\subsection{Functional Performance Requirements}

Functional performance requirements for each section of the test are listed below. Further development of the GeoSiphon Treatment system is anticipated in FY99.

\subsubsection{Ex-Situ Treatment Media Evaluation}

- Design of the treatment train shall allow for easy exchange of canisters and configurations.

- The ex situ treatment systems shall be designed to withstand pressure loss associated with the treatment media uptake capacity.

- The ex situ treatment system shall be designed for ambient conditions.

- The ex situ system shall have the capacity to operate from 0 to 10 gallons per minute.

- The maximum operating pressure of the system shall be 15 psig.

- Materials of construction shall be compatible with the treatment media and the groundwater characteristics.

\subsubsection{D-Area GeoSiphon Treatment System Conceptual Design}

- The GeoSiphon treatment system shall be designed for ambient conditions.

- The GeoSiphon treatment system shall have the capacity to operate at 10 gallons per minute or less.

- Materials of construction shall be compatible with the treatment media and the groundwater characteristics.

- The design of the treatment system shall allow for easy exchange/replacement of media.

- The design shall utilize natural hydraulic driving forces to induce flow for remediation.

\section{D-AREA EX SITU TREATMENT MEDIA EVALUATION}

\subsection{Test Objectives/Rationale}

The D-Area Coal Pile Runoff Basin (CPRB) groundwater is contaminated with low $\mathrm{pH}$, iron, aluminum, sulfate, chromium, and nickel with lesser amounts of trichloroethylene, cadmium, and lead. The CPRB requires remediation per the CERCLA process in accordance with the Federal Facility Agreement (FFA) for the Savannah River Site. An investment at the CPRB was made during fiscal years 1994 through 1996 in the design, installation, and testing of a barrier wall system within the most contaminated portion of the D-Area CPRB plume. The objective of this testing was to evaluate various permeable treatment media or combinations of media for the treatment of this groundwater. The results of this effort will be used to design, construct and test a treatment system for the metals contaminated groundwater at the CPRB in conjunction with the existing barrier wall system. 


\subsection{Technology/Process Description}

\subsubsection{Ex Situ Treatment Media Evaluation}

The ex situ treatment media evaluation was a permeable treatment media treatability study conducted at the CPRB utilizing the actual contaminated groundwater. The following treatment media were used in the evaluation: limestone, rock phosphate (apatite), granular cast iron, peat (Sphagnum Moss), zeolite (Clinoptilolite), potassium phosphate, sodium phosphate, ammonium phosphate, calcium phosphate and potassium phosphate using limestone and/or phosphate rock as a reactive matrix. The field tests were conducted at the D-Area CPRB utilizing the existing barrier wall system (DIW-1-2, Interceptor Well) and surrounding monitoring wells to extract the contaminated groundwater for above grade testing. The treatment media or reactive media for the field studies was contained in in-line canisters, and samples were taken for chemical analysis to determine the effectiveness of each treatment process. The treated groundwater was subsequently released to the D-Area CPRB. The information produced from the treatability study will feed into the subsequent D-Area GeoSiphon Treatment System design, installation and testing using the existing barrier wall system.

\subsubsection{Existing Barrier Wall System}

A demonstration of the barrier wall system was conducted adjacent to the CPRB. The barrier wall system utilizes trench and guidebox construction techniques to install high-density polyethylene (HDPE) vertical barriers below grade. In its application at the CPRB, a vertical HDPE barrier was installed and combined with a groundwater recovery system in the same trench to produce an interceptor well. The D-Area barrier wall/interceptor well (DIW-1) was installed at the northwest corner of the D-Area CPRB to a depth of 30 feet. The barrier wall/interceptor well bottom does not tie into the underlying aquitard, but it is approximately 30 feet above the aquitard. This produced a hanging or partially penetrating barrier wall.

The barrier wall/interceptor well has an innovative design which maximizes the collection of upgradient contaminated groundwater while minimizing the collection of downgradient groundwater. It also provides for a more even distribution of groundwater collection across the entire width of the contaminant plume. The segregation of upgradient and downgradient groundwater is achieved by the installation of a vertical high density polyethylene (HDPE) membrane as part of the well immediately downgradient from the gravel pack and well screens. Even distribution of groundwater collection across the plume width is achieved in two ways. First, it is achieved by installation of a continuous gravel pack along the entire length of the HDPE membrane. The gravel pack provides complete groundwater interception and collection along its entire depth and length. 
Second, by installation of horizontal slotted drainage pipe within the gravel pack parallel to the HDPE membrane, which tie into a central well sump. These horizontal drainage pipes flatten out and extend the drawdown curve throughout the gravel pack, which facilitates flow from the ends toward the central, vertical well screens. Multiple vertical well screens all attached to one well sump, along with the horizontal drainage pipes, provide centralized collection of the groundwater for extraction purposes. Two vertical well screens are extended to the surface with casing for groundwater extraction

The existing D-Area barrier wall system produces mounding of the groundwater upgradient of the wall, which produces a significant head differential between the groundwater upgradient of the wall and the adjacent discharge stream. This head differential can be utilized to drive a GeoSiphon treatment system.

\subsubsection{GeoSiphon Treatment System Conceptual Design}

GeoSiphon Cells are passive groundwater treatment systems which utilize siphon flow between two points of natural head difference to induce greater than natural groundwater flow through a treatment system without the requirement for external energy input. A treatment system used in GeoSiphon Cells can include permeable treatment materials, aeration, chemical addition, etc.

The conceptual GeoSiphon Cell configuration includes extraction of the contaminated water from the mound created by the barrier wall system by siphon to a downgradient treatment system which may consist of an oxidizing step such as aeration followed by a permeable treatment media or treatment media combination. The siphon flow will be induced by the natural hydraulic head difference between the mound upgradient of the wall and the adjacent D-Area discharge stream, which is a lower hydraulic head. Further development of this concept is anticipated in FY99.

\subsection{Technology Applications}

The 489-D D-Area Coal Pile Runoff Basin (CPRB) and the 488-D D-Area Ash Basin are listed RCRA/CERCLA units as outlined in the Federal Facility Agreement (FFA) for the Savannah River Site (SRS). These sites are currently entering the Remedial Investigation/Baseline Risk Assessment phase of the CERCLA process. The SRS Environmental Restoration Department (ERD) is seeking low maintenance and low operating cost remediation alternatives other than pump and treat for both the CPRB and the Ash Basin. The GeoSiphon treatment system is a low maintenance and low operating cost alternative to pump and treat. As such ERD has assumed that this technology will be applied at both the CPRB and the 488-D Ash Basin, if positive results are obtained from the work conducted under this project. The work conducted under this project will be 
incorporated into the CERCLA process for the CPRB and Ash Basin through preparation of a Treatability Study Test Plan and a subsequent Treatability Study Report. The information produced and documented will feed into the CERCLA Feasibility Study and the subsequent CERCLA Proposed Plan for the CPRB and Ash Basin and will be made available to the public.

ERD is an integral part of the D-Area Remediation Technical Team. All work associated with this project has been conducted in conjunction with ERD to assure that work will meet the end users needs and conform to regulatory expectations.

Additionally, ERD demonstrated their strong support for passive in situ treatment systems by providing funding in FY97 and in FY98 to fund the installation and testing of a GeoSiphon cell in the contaminated groundwater at the SRS TNX Area. The TNX Demonstration GeoSiphon Cell was installed in July 1997 and Phase I testing occurred from $8 / 5 / 97$ to $2 / 5 / 98$. Phase II testing began on 6/18/98. To further elaborate ERDs commitment for a passive treatment system, a second GeoSiphon cell was installed in the TNX area in September 1998. Lessons learned and end user needs identified from the GeoSiphon Cell work is directly transferable to the work conducted under this project.

This project also addresses the Savannah River Site (SRS) need for in-situ or exsitu ground water treatment technologies for hazardous constituents in unconsolidated subsurface sediments (i.e. sandy/clayey soils) associated with the metals and sulfate contamination in the ground water at D-Area. The results of this treatability study will be applicable to other metals contaminated sites throughout the DOE complex.

\subsection{Technology Benefits}

The benefits of this technology include:

- Evaluation of various permeable treatment media or combinations of media for the treatment of the D-Area CPRB contaminated groundwater.

- Utilization of the existing barrier wall system as part of a treatment system for the D-Area CPRB metals contaminated groundwater. The existing barrier wall system represents a significant past Office of Science and Technology (OST) investment.

- Treatment within the most heavily contaminated portion of the D-Area CPRB plume.

- Removable treatment options so that heavy metals can be collected and recycled.

- Potentially accelerate clean up, can be applied to a broader range of site conditions, and can increase treatment media longevity and effectiveness.

- Minimize media armoring and/or cementation, and formation pluggage.

- Utilize natural hydraulic driving forces to induce flow for remediation. 
- Directly meeting the needs of the EM-40 program (Environmental Restoration).

- While costs savings over the baseline can not yet be determined, it is anticipated that a passive treatment system will significantly reduce the operation and maintenance costs associated with the presumptive baseline, pump and treat system.

\section{TEST STRATEGY AND DESIGN}

\subsection{Test Campaigns}

The ex situ treatment media evaluation is a permeable treatment media treatability study conducted at the CPRB utilizing the actual contaminated groundwater. The following treatment media were used in the evaluation: limestone, rock phosphate (apatite), granular cast iron, peat (Sphagnum Moss), zeolite (Clinoptilolite), potassium phosphate, sodium phosphate, ammonium phosphate, calcium phosphate and potassium phosphate using limestone and/or phosphate rock as a reactive matrix. The field tests were conducted at the D-Area CPRB utilizing the barrier wall system (DIW-1-2, Interceptor Well) and surrounding monitoring wells to extract the contaminated groundwater for above grade testing. The treatment media or reactive media was contained in in-line canisters, and samples were taken for chemical analysis to determine the effectiveness of each treatment process. The treated groundwater was subsequently released to the D-Area CPRB. Three field runs/test campaigns and four laboratory tests were conducted. The information produced from this treatability study will be incorporated into the subsequent D-Area GeoSiphon Treatment System design, installation and testing.

\subsubsection{Field Run \#1 (2/98-3/98)}

Field Run 1 was set up as a dual media reactive contact system in series with sludge settling capability in-between. This was the initial field concept for the treatment of the groundwater.

\subsubsection{Field Run \#2 (4/98)}

Field Run 2 was designed as a non-contact system. This consisted of a single media system in which potable water was pumped through the media and mixed with the contaminated water in a second canister for treatment. A non-contact configuration was used in order to minimize the armoring and cementation of the treatment media that occurred in Field Run \#1.

\subsubsection{Field Run \#3 (7/98-8/98)}

Based on the preliminary results of Lab Test \#1 and visual observations of Lab Test \#2, an aggregate capable of facilitating a reaction and enabling precipitation was suggested for our waste stream. Field Run 3 was designed as a reactive contact system. It consisted of a single media system in which a solution equilibrated with treatment media was pumped 
in tandem with the contaminated groundwater into a canister and allowed to react on the selected aggregate.

\subsubsection{Lab Test \#1 (5/98)}

Field Run \#2 suggested that potable water was not capable of solubilizing sufficient amounts of phosphate for precipitation. This laboratory test was performed to determine the effects of more soluble phosphate compounds with the metals contaminated groundwater from the Coal Pile Runoff Basin. Experiments in this test utilized various concentrations of sodium phosphate and potassium phosphate as well as phosphate rock media solutions at varying $\mathrm{pHs}$.

\subsubsection{Lab Test \#2 (6/98)}

This laboratory test was performed to determine the effects of more soluble phosphate compounds with the metals contaminated groundwater from the Coal Pile Runoff Basin. This experiment was a continuation of the first laboratory experiment. Experiments in this test utilized various concentrations of ammonium phosphate and calcium phosphate as well as using phosphate rock or limestone as reactive media matrices at varying potassium phosphate concentrations.

\subsubsection{Lab Test \#3 (7/98)}

This laboratory test occurred simultaneously as Field Run \#3 and was performed to determine the effects of treatment media particle size with the metals contaminated groundwater from the Coal Pile Runoff Basin. Experiments in this test utilized crushed limestone and/or phosphate rock at various ratios and contact times.

\subsubsection{Lab Test \#4 (9/98)}

Longer contact times tended to enhance the removal rates in Lab Test \#3. This phenomena suggested that oxidation may be occurring with time. Laboratory Test \#4 was performed to determine the effects of aeration of the metals contaminated groundwater prior to treatment. Experiments in this test utilized limestone powder or potassium phosphate solutions with the groundwater.

\subsection{Experimental Design}

\subsubsection{Field Run \#1}

Field Run 1 was constructed as a dual media system in series with sludge settling capability in between each of the two canisters in the system. Four trains /treatment systems were operated during Field Run 1 with the media combinations identified in Table 2. Each train consisted of a peristaltic pump, a 4" diameter by 5.5' long PVC pipe column for media \#1, a 15 gallon sludge knockout drum, a 4" diameter by 5.5' long PVC pipe 
column for media \#2, and a discharge line to the D-Area CPRB. Sampling valves were provided in various locations before, after, and throughout each column. See Figure 2 and for a schematic of the system.

Table 2

Field Run 1 -Treatment Media Evaluation - Media Combinations/Filter Configuration

\begin{tabular}{||l|l|l|l|l|}
\hline Train & Canister \#1 & Canister \#2 & Filter Configuration \\
\hline 1 & crushed limestone & peat (Sphagnum Moss) & Filter fabric at the inlet and outlet of canisters 1 and 2 \\
\hline 2 & crushed limestone & zeolite (Clinoptilolite) & Filter fabric at the inlet and outlet of canisters 1 and 2 \\
\hline 3 & crushed limestone & granular cast iron & Filter fabric at the inlet and outlet of canisters 1 and 2 \\
\hline 4 & phosphate rock & granular cast iron & Filter fabric at the inlet and outlet of canisters 1 and 2 \\
\hline
\end{tabular}

\subsubsection{Field Run \#2}

Field Run 2 was designed as a non-contact system. Two treatment trains operated with the media combinations listed in Table 3. This consisted of a single media system in which potable water was pumped through the media and mixed with the contaminated water in a second canister for treatment. Each system consists of a peristaltic pump, connected to a 4" diameter by $5.5^{\prime}$ long PVC pipe column for the treatment media. A peristaltic pump, connected to a second 4" diameter by 5.5' long PVC pipe column packed with 3" Cascade Mini Rings (where the contaminated groundwater was introduced), and a discharge line to the D-Area CPRB. Sampling valves were provided in various locations throughout each system. See Figure 3 for a schematic of the system.

Table 3

Field Run 2 -Treatment Media Evaluation - Media Combinations/ Filter Configuration

\begin{tabular}{||l|l|l|l|}
\hline \hline Train & Canister \#1 & Canister \#2 & Filter Configuration \\
\hline 1 & crushed limestone & Cascade Mini Rings & $\begin{array}{l}\text { Filter fabric at the bottom (inlet) of canister 1 and at the bottom } \\
\text { (outlet) of canister 2 }\end{array}$ \\
\hline 2 & Phosphate rock & Cascade Mini Rings & $\begin{array}{l}\text { Filter fabric at the bottom (inlet) of canister 1 and at the bottom } \\
\text { (outlet) of canister 2 }\end{array}$ \\
\hline
\end{tabular}




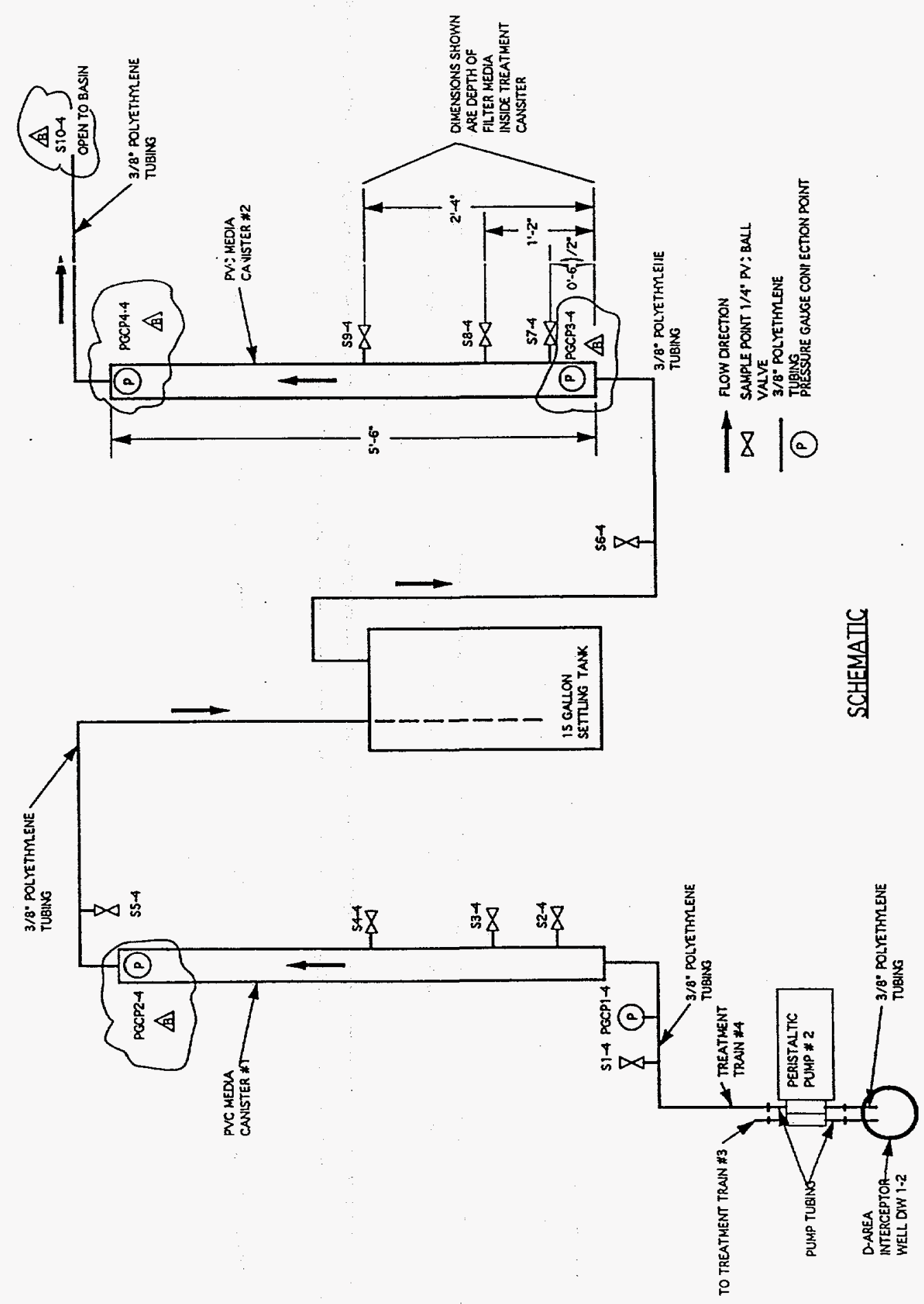

Figure 2 - Schematic Representation of Field Run \#1 


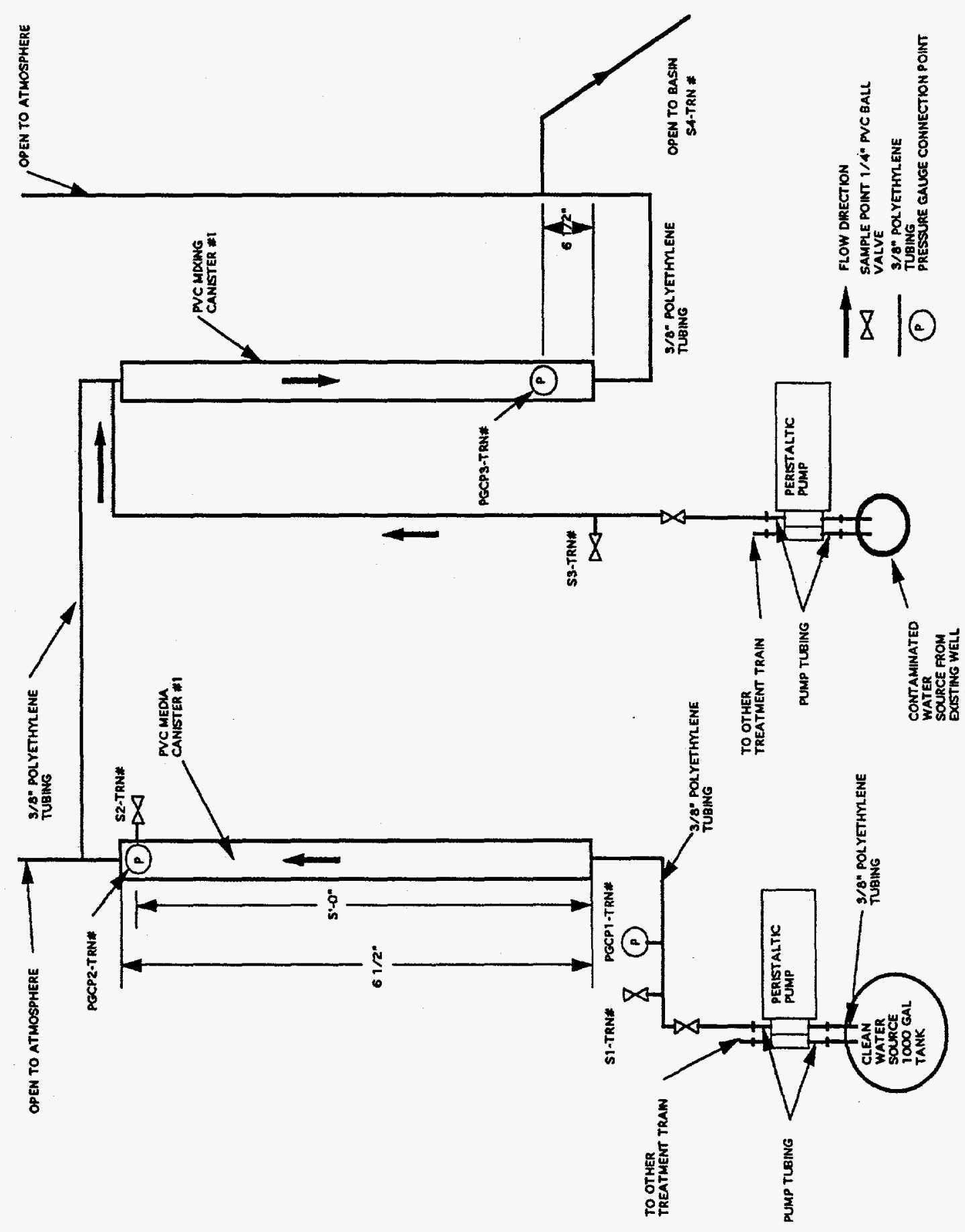

Figure 3-Schematic Representation of Field Run \#2 


\subsubsection{Field Run \#3}

Field Run 3 was designed as a reactive contact system. Two treatment trains were tested and media combinations are listed in Table 4. The treatment trains consisted of a single media system in which a treatment media solution is pumped in tandem with the contaminated groundwater into a canister and allowed to react on the selected aggregate. Each system consisted of a peristaltic pump, connected to a 4 " diameter by 5.5 ' long PVC pipe column for the treatment media solution, a peristaltic pump, connected to the same 4" diameter by 5.5' long PVC pipe column for the contaminated groundwater, and a discharge line to the D-Area CPRB. See Figure 4 for a schematic of the system.

\section{Table 4}

Field Run 3 -Treatment Media Evaluation - Media Combinations/ Filter Configuration

\begin{tabular}{||l|l|l|l||}
\hline Train & Canister \#1 & Canister \#2 & Filter Configuration \\
\hline 1 & crushed limestone & N/A & $\begin{array}{l}\text { Two opposed layers of geonet at the bottom (inlet) of the } \\
\text { canister }\end{array}$ \\
\hline 2 & $\begin{array}{l}\text { crushed limestone/ } \\
\text { (90 ml/gal) } \\
\text { phosphate rock }\end{array}$ & N/A & $\begin{array}{l}\text { Two opposed layers of geonet at the bottom (inlet) of the } \\
\text { canister }\end{array}$ \\
\hline
\end{tabular}

\subsubsection{Lab Test \#1}

This laboratory test was primarily designed by determining the stoichiometric concentrations of sodium and potassium phosphate compounds required for three separate groundwater contaminated concentrations within the D-Area Coal Pile Runoff Basin groundwater plume. Proportions of $50 \%, 100 \%$ and $150 \%$ the theoretical amount were tested for the most soluble compound, potassium phosphate. Phosphate rock solubility was also tested by varying the $\mathrm{pH}$ to determine any effects in a non-contact configuration. The concentrations that were used in this experiment are shown in the Table 5. 

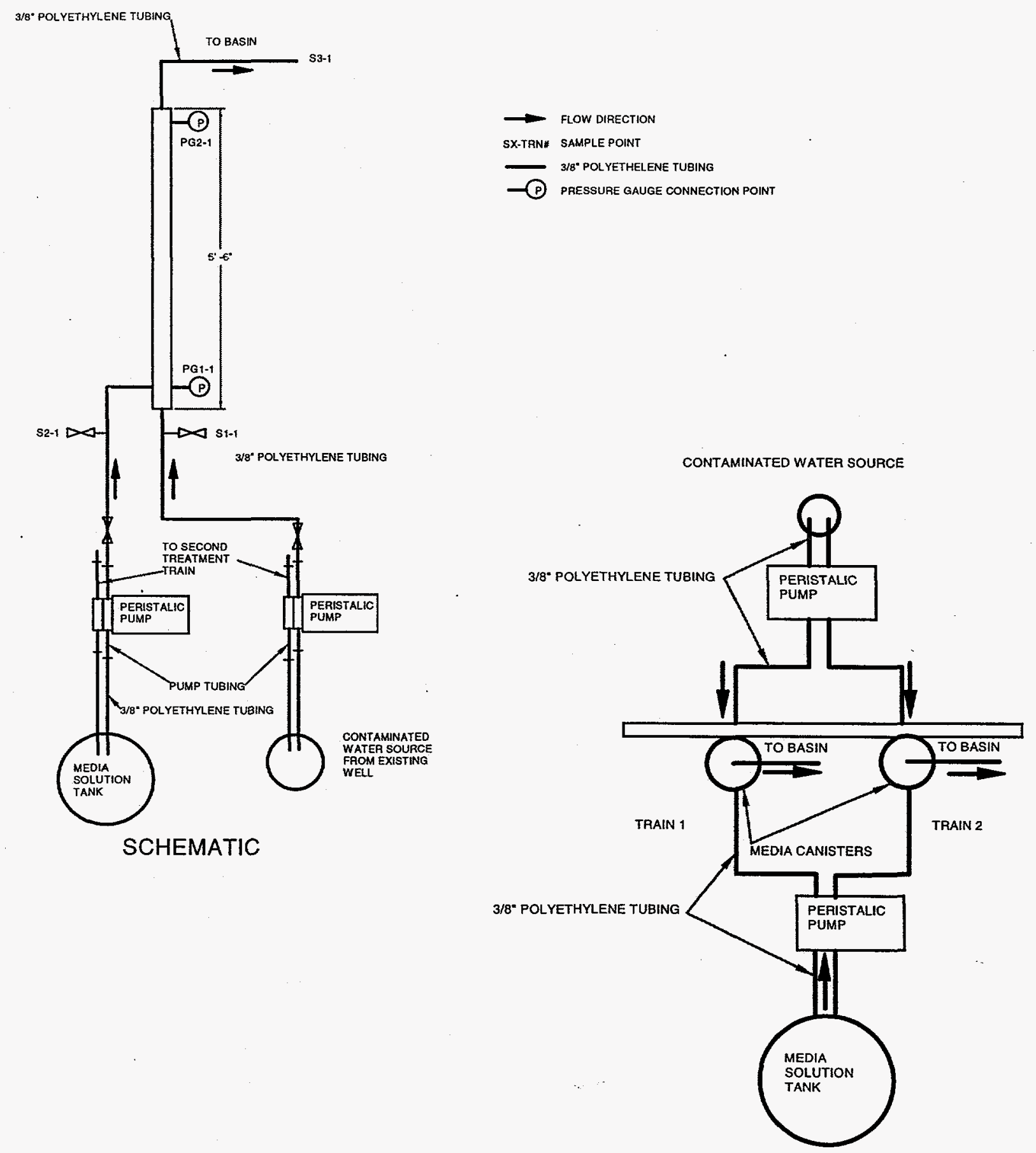

PLAN VIEW

Figure 4 - Schematic Representation of Field Run \#3 
Table 5

\section{Laboratory Test Using More Soluble Phosphate Media}

\begin{tabular}{|c|c|c|c|}
\hline Well & DCB-21A & DCB-10 & DCB-4A \\
\hline $\begin{array}{l}\text { Typical } \\
\text { Contaminant } \\
\text { Concentration } \\
(\mathrm{mg} / \mathrm{l})\end{array}$ & $\begin{array}{l}\text { Al: } 470 \\
\text { Ca: } 130 \\
\text { Cr: } 0.5 \\
\text { Fe: } 1360 \\
\text { Mg: } 135 \\
\text { Mn: } 16 \\
\text { Ni: } 3.3 \\
\end{array}$ & $\begin{array}{l}\text { Al: } 227 \\
\mathrm{Ca}: 82 \\
\mathrm{Cr}: 0.2 \\
\mathrm{Fe}: 216 \\
\mathrm{Mg}: 49 \\
\mathrm{Mn}: 8 \\
\mathrm{Ni}: 1.1 \\
\end{array}$ & $\begin{array}{ll}\text { Al: } & 46 \\
\text { Ca: } & 71 \\
\text { Cr: } & 0.02 \\
\text { Fe: } & 20 \\
\text { Mg: } 43 \\
\text { Mn: } 3 \\
\text { Ni: } 0.4 \\
\end{array}$ \\
\hline \multirow{3}{*}{$\begin{array}{l}\text { Potassium } \\
\text { Phosphate, } \\
\text { dibasic } \\
\text { Concentrations } \\
(\mathrm{mg} / \mathrm{l}) \\
\end{array}$} & $18.75 \mathrm{~g} / 1$ & $6 \mathrm{~g} / \mathrm{l}$ & $1.8 \mathrm{~g} / 1$ \\
\hline & $12.5 \mathrm{~g} / \mathrm{l}$ & $4 \mathrm{~g} / 1$ & $1.2 \mathrm{~g} / 1$ \\
\hline & $6.25 \mathrm{~g} / 1$ & $2 \mathrm{~g} / 1$ & $0.6 \mathrm{~g} / 1$ \\
\hline $\begin{array}{l}\text { Sodium Phosphate, } \\
\text { dibasic } \\
\text { Concentration } \\
(\mathrm{mg} / \mathrm{l})\end{array}$ & $10.2 \mathrm{~g} / 1$ & $3.2 \mathrm{~g} / 1$ & $1.0 \mathrm{~g} / 1$ \\
\hline \multirow[t]{2}{*}{ Phosphate Rock } & $\begin{array}{l}\text { Saturated in } 4.5 \mathrm{pH} \\
\text { water }\end{array}$ & $\begin{array}{l}\text { Saturated in } 4.5 \mathrm{pH} \\
\text { water }\end{array}$ & $\begin{array}{l}\text { Saturated in } 4.5 \mathrm{pH} \\
\text { water }\end{array}$ \\
\hline & $\begin{array}{l}\text { Saturated in } 5.5 \mathrm{pH} \\
\text { water }\end{array}$ & $\begin{array}{l}\text { Saturated in } 5.5 \mathrm{pH} \\
\text { water }\end{array}$ & $\begin{array}{l}\text { Saturated in } 5.5 \mathrm{pH} \\
\text { water }\end{array}$ \\
\hline
\end{tabular}

\subsubsection{Lab Test \#2}

This laboratory test was primarily designed by determining the stoichiometric concentrations of ammonium and calcium phosphate compounds required for three different groundwater contaminated concentrations within the D-Area Coal Pile Runoff Basin groundwater plume. Proportions of 50\%, 100\% and $150 \%$ of the theoretical amount were tested using ammonium phosphate, while a saturated solution of calcium phosphate was used. Additionally, phosphate rock and washed limestone were tested as a reactive matrix with solutions of groundwater and potassium phosphate. The concentrations that were used in this experiment are shown in the Table 6. 
Table 6

\section{Laboratory Test Using More Soluble Phosphate Media (Con't)}

\begin{tabular}{|c|c|c|c|}
\hline Well & DCB-21A & DCB-10 & DCB-4A \\
\hline $\begin{array}{l}\text { Typical } \\
\text { Contaminant } \\
\text { Concentration } \\
(\mathrm{mg} / \mathrm{l})\end{array}$ & $\begin{array}{l}\text { Al: } 470 \\
\text { Ca: } 130 \\
\text { Cr: } 0.5 \\
\text { Fe: } 1360 \\
\text { Mg: } 135 \\
\text { Mn: } 16 \\
\text { Ni: } 3.3 \\
\end{array}$ & $\begin{array}{l}\text { Al: } 227 \\
\text { Ca: } 82 \\
\text { Cr: } 0.2 \\
\text { Fe: } 216 \\
\text { Mg: } 49 \\
\text { Mn: } 8 \\
\text { Ni: } 1.1\end{array}$ & $\begin{array}{l}\text { Al: } 46 \\
\mathrm{Ca}: 71 \\
\mathrm{Cr}: 0.02 \\
\text { Fe: } 20 \\
\mathrm{Mg}: 43 \\
\mathrm{Mn}: 3 \\
\mathrm{Ni}: 0.4 \\
\end{array}$ \\
\hline $\begin{array}{l}\text { Ammonium } \\
\text { Phosphate, } \\
\text { dibasic } \\
\text { Concentration } \\
(\mathrm{mg} / \mathrm{l}) \\
\end{array}$ & $14.22 \mathrm{~g} / 1$ & $4.49 \mathrm{~g} / \mathrm{l}$ & $1.33 \mathrm{~g} / 1$ \\
\hline $\begin{array}{l}\text { Calcium } \\
\text { Phosphate, } \\
\text { monobasic } \\
\text { Concentration } \\
(\mathrm{mg} / \mathrm{l}) \\
\end{array}$ & $18.0 \mathrm{~g} / 1$ & $18.0 \mathrm{~g} / 1$ & $18.0 \mathrm{~g} / 1$ \\
\hline $\begin{array}{l}\text { Phosphate } \\
\text { Rock }(\mathrm{g})+ \\
\text { Potassium } \\
\text { phosphate, } \\
\text { dibasic }(\mathrm{g} / \mathrm{l})\end{array}$ & $\begin{array}{l}10 \% \text { by volume } \\
\text { of phosphate } \\
\text { rock }+18.75 \mathrm{~g} / \mathrm{l} \\
\text { of potassium } \\
\text { phosphate, } \\
\text { dibasic solution }\end{array}$ & $\begin{array}{l}\text { l0\% by volume of } \\
\text { phosphate rock }+6.0 \\
\text { g/l of potassium } \\
\text { phosphate, dibasic } \\
\text { solution }\end{array}$ & $\begin{array}{l}10 \% \text { by volume of } \\
\text { phosphate rock }+1.8 \\
\text { g/l of potassium } \\
\text { phosphate, dibasic } \\
\text { solution }\end{array}$ \\
\hline $\begin{array}{l}\text { Washed } \\
\text { Limestone } \\
(\mathrm{g})+ \\
\text { Potassium } \\
\text { phosphate, } \\
\text { dibasic }(\mathrm{g} / \mathrm{l})\end{array}$ & $\begin{array}{l}18.75 \mathrm{~g} / \mathrm{L} \text { of } \\
\text { potassium } \\
\text { phosphate } \\
\text { dibasic solution } \\
\text { saturating the } \\
\text { pore space. }\end{array}$ & $\begin{array}{l}6.0 \mathrm{~g} / \mathrm{L} \text { of potassium } \\
\text { phosphate dibasic } \\
\text { solution saturating } \\
\text { the pore space. }\end{array}$ & $\begin{array}{l}1.8 \mathrm{~g} / \mathrm{L} \text { of potassium } \\
\text { phosphate dibasic } \\
\text { solution saturating } \\
\text { the pore space. }\end{array}$ \\
\hline
\end{tabular}

\subsubsection{Lab Test \#3}

This laboratory test was primarily to determine whether particle size and contact time effects the metals remediation within the D-Area Coal Pile Runoff Basin groundwater plume. Crushed particles of limestone or phosphate rock were less than $0.5 \mathrm{~mm}$ in diameter. The concentrations that were used in this experiment are shown in the Table 7. 
Table 7

\section{Laboratory Testing with Crushed Limestone and Phosphate Rock}

\begin{tabular}{|l|l|}
\hline Well & DIW-1-2 \\
\hline Typical Contaminant Concentration & Al: 78.7 \\
& Ca: 50.0 \\
& Cr: 4.7 \\
& Fe: 36.7 \\
& Mg: 26.5 \\
& Mn: 6.1 \\
& Ni: 1.1 \\
\hline & \\
\hline Crushed Limestone & 10 vol\% with no mix, settle 24 hours \\
& 10 vol\% with 5 min. mix \\
& 10 vol\% with 10 min. mix \\
& 20 vol\% with 5 min. mix \\
& 20 vol\% with 10 min. mix \\
\hline & \\
\hline Ground phosphate rock & 10 vol\% with no mix, settle 24 hours \\
& 10 vol\% with 5 min. mix \\
& 10 vol\% with 10 min. mix \\
& 20 vol\% with 5 min. mix \\
& 20 vol\% with 10 min. mix \\
\hline
\end{tabular}

\subsubsection{Lab Test \#4}

This laboratory run was performed to determine the affects of aeration on the treatment of the contaminated groundwater. Limestone powder less than $0.5 \mathrm{~mm}$ in diameter and potassium phosphate solutions were used. The concentrations that were used in this experiment are shown in the Table 8.

Table 8

Laboratory Testing with Crushed Limestone, Potassium Phosphate, and Aerated Groundwater

\begin{tabular}{|l|l|l|}
\hline Well & DCB-21A & DIW-1-2 \\
\hline Typical Contaminant Concentration & Al: 60.7 & Al: 78.7 \\
(mg/l) & Ca: 73.8 & Ca: 50.0 \\
& Cr: 0.1 & Cr: 4.7 \\
& Fe: 102.3 & Fe: 36.7 \\
& Mg: 42.6 & Mg: 26.5 \\
& Mn: 3.1 & Mn: 6.1 \\
& Ni: 0.7 & Ni: 1.1 \\
\hline Crushed Limestone & 20 vol\% & 20 vol\%. \\
& & \\
\hline Potassium Phosphate & $1.7 \mathrm{~g} / \mathrm{L}$ & $1.8 \mathrm{~g} / \mathrm{L}$ \\
& & \\
\hline
\end{tabular}




\subsection{Equipment, Materials \& Methods}

The following field equipment was used during the course of the project:

\begin{tabular}{ll} 
Equipment & Measurement \\
\hline OAKTON pH/Eh/ ${ }^{\circ} \mathrm{C}$ Meter & $\mathrm{pH} / \mathrm{Eh} /$ Temperature \\
ORION Model 250A & $\mathrm{pH}$ \\
YSI Model 58 Dissolved Oxygen Meter & $\mathrm{DO}$ \\
YSI Model 3800 and 3820 & $\mathrm{pH}, \mathrm{Eh}, \mathrm{DO}$ \\
HYDROLAB Model Data Sonde II & $\mathrm{pH}, \mathrm{Eh}, \mathrm{DO}$ \\
PSI TRONIX PG 5000 Digital Pressure Gauge & Pressure
\end{tabular}

\subsubsection{Field Run \#1}

To allow for continuous operation, a diesel generator was utilized to power the pumps and sampling equipment. All system plumbing was assembled using $3 / 8$ " polyethylene tubing and $3 / 8$ " KYNAR® fittings (or equal). Pumping at the required low flow rates was delivered by Master Flex® peristaltic pumps with variable speed pump drives and pump heads. Canisters (4" diameter by 5.5' long) were constructed from schedule 40 PVC pipe and fittings. 1/4" PVC sampling valves were provided in various locations before, after, and were installed throughout each column as need to assist in determining media breakthrough curves. A filter fabric consisting of a nonwoven needle punched polyester geotextile fabric heatlaminated to both sides of a high-density polyethylene (HDPE) geonet was installed in the configuration listed in Table 2. 15-gallon polyethylene sludge knockout drums were used between the treatment canisters.

Once the treatment trains were constructed, a potable water flush was performed to ensure the systems integrity. All potable water was drained and the appropriate safety and system walk downs were also performed prior to each field run. The test well, DIW-1-2, was purged at a rate of 5 gallons per minute for approximately three days prior to the field run.

Figure 2 depicts the test equipment layout and specific equipment for the field run. Peristaltic pumps capable of low flows $(50-200 \mathrm{ml} / \mathrm{min})$ through the treatment trains were utilized. Flow was measured from the discharge using a graduated cylinder and stopwatch to calibrate the flow, and flow readings from the peristaltic pumps were collected and recorded. Initial and weekly sampling of inlet and outlet canister sampling ports were conducted. Groundwater samples were collected from the inlets and outlets of each treatment canister. Field measurements of $\mathrm{pH}, \mathrm{Eh}$, and DO were collected. Headspace samples for VOC analyses were taken on the initial inlet sampling port only. 


\subsubsection{Field Run \#2}

Materials of construction were the same as those used in Field Run\#1. The same filter fabric as used in Field Run \#1 was installed in the configurations listed in Table 3 for Field Run \#2. No 15-gallon polyethlyene drums were used in this run. Figure 3 depicts the test equipment layout and specific equipment for the field run.

Once treatment trains were constructed, system integrity checks, walk downs and purging of the test well (DIW-1-2) were completed as in Field Run \#1.

\subsubsection{Field Run \#3}

Materials of construction were the same as those used in Field Run\#1. Instead of the geotextile fabric and HDPE geonet used in Runs 1 and 2, two layers of only the HDPE geonet mesh were on the inlet of each canister. This was used to provide an even distribution of the influent across the entire cross section of the canister and eliminate plugging problems associated with the fabric itself. Five gallon catch tanks with screened bottoms and a layer of filter fabric were set up at the discharge points in the basin. Figure 4 depicts the test equipment layout and specific equipment for the field run.

Once treatment trains were constructed, system integrity checks, walk downs and purging of the test well (DIW-1-2) were completed as in Field Runs \#1 and \#2.

\subsubsection{Lab Test \#1}

This ex situ treatment media evaluation laboratory experiment consisted of $50 \mathrm{ml}$ graduated centrifuge tubes that were used for test observations and measurements. The test wells, DCB-21A, DCB-10 and DCB-4A were purged for approximately three well volumes prior to collection of samples for experimentation. Approximately $1 \mathrm{~L}$ of groundwater was collected in two 500-ml sterile flasks from the wells listed above. Headspace was minimized during sample collection. Potassium phosphate and sodium phosphate solutions were prepared as described in Table 5 using Nanopure water. Aliquots of the Nanopure water were adjusted with hydrochloric acid for the phosphate rock solutions to the pHs listed in Table 5. A 50\% by weight mixture of rock phosphate and $\mathrm{pH}$ adjusted Nanopure waters were prepared as listed in Table 5 and allowed to saturate for 24 hours. Observations were recorded.

Equal volumes of the contaminated groundwater and treatment media solutions listed in Table 5 were mixed in the $50 \mathrm{ml}$ graduated centrifuge tubes and allowed to settle for 24 hours in a manner that minimized exposure to air. The samples were monitored after mixing in order to determine visual precipitate formation. After the 24-hour settling period, 
the precipitate characteristics (i.e. color, texture, volume, etc.) were recorded.

\subsubsection{Lab Test \#2}

This ex situ treatment media evaluation laboratory experiment consisted of $50 \mathrm{ml}$ graduated centrifuge tubes that were used for each test for. observations and measurements. Groundwater from wells DCB-21A, DCB-10 and DCB-4A that were collected during the previous experiment were used. Ammonium phosphate and calcium phosphate solutions were prepared as described in Table 6 using Nanopure water. Enough limestone was added to the specific centrifuge tubes in order to fill the tube. Five milliliters of phosphate rock was added to their specific tubes for testing. Equal volumes of groundwater and potassium phosphate solutions were mixed just before sampling and then transferred to limestone and phosphate rock centrifuge tubes. Observations were recorded.

Equal volumes of the contaminated groundwater and ammonium phosphate or calcium phosphate solutions were mixed in the $50 \mathrm{ml}$ graduated centrifuge tubes and allowed to settle for 24 hours in a manner that minimized exposure to air. The samples were monitored after mixing in order to determine visual precipitate formation. After the 24-hour settling period, the precipitate characteristics (i.e. color, texture, volume, etc.) were recorded.

Reactive matrixed test tubes were mixed with groundwater/potassium phosphate solutions and allowed to settle for 24 hours in a manner that minimized exposure to air. After the 24-hour settling period, the precipitate characteristics (i.e. color, texture, volume, etc.) were recorded. Aqueous portions were fractioned and analytical measurements were obtained.

\subsubsection{Lab Test \#3}

This ex situ treatment media evaluation laboratory experiment consisted of $50 \mathrm{ml}$ graduated centrifuge tubes. The tubes were used for test observations, analyte measurements, $\mathrm{pH}$ measurements, and $\mathrm{Eh}$ measurements. The test well, DIW-1-2, was purged for approximately three days prior to collection of samples for experimentation.

Approximately $500 \mathrm{ml}$ was collected in sterile flasks from the well such that no headspace remained. All phosphate solutions were prepared as described in Table 7 using Nanopure water.

Volumes of the contaminated groundwater and treatment media were mixed according to Table 7 in the $50 \mathrm{ml}$ graduated centrifuge tubes and allowed to settle for in a manner that minimizes exposure to air. Only those samples listed as such were allowed to settle for 24 hours. The samples were monitored after mixing in order to note the visual precipitate 
formation. After the settling period, the precipitate characteristics (i.e. color, texture, volume, etc.) were noted.

\subsubsection{Lab Run \#4}

This ex situ treatment media evaluation laboratory experiment consisted of $50 \mathrm{ml}$ graduated centrifuge tubes. The tubes were used for test observations, and analyte measurements. The test wells, DIW-1-2 and DCB-21A, were purged for approximately three well volumes prior to collection of samples for experimentation. Approximately $250 \mathrm{ml}$ was collected in sterile flasks from the well such that no headspace remained. All phosphate solutions were prepared as described in Table 8 using Nanopure water.

Initial samples were collected on the groundwater and air was bubbled through each flask for approximately two hours. Equal volumes of the contaminated groundwater and potassium phosphate solutions listed in Table 8 were mixed in the $50-\mathrm{ml}$ graduated centrifuge tubes and allowed to settle. Limestone and groundwater were mixed in the $50 \mathrm{ml}$ graduated centrifuge tubes and allowed to settle. The samples were monitored after mixing in order to note the visual precipitate formation. After the settling period, the precipitate characteristics (i.e. color, texture, volume, etc.) were noted.

\subsection{Sampling and Analysis}

The following table represents the analytical groups were measured during the testing phase:

Table 9

Analytical Groups

\begin{tabular}{|c|c|}
\hline Analytical Group & Analytes \\
\hline 1 & $\begin{array}{l}\text { RCRA Suite of Metals: silver, aluminum, boron, barium, } \\
\text { calcium, cadmium, cerium, cobalt, chromium, copper, iron, } \\
\text { lanthanum, lithium, magnesium, manganese, molybdenum, } \\
\text { sodium, nickel, phosphorus, lead, silica, tin, strontium, titanium, } \\
\text { vanadium, zinc, and zirconium }\end{array}$ \\
\hline 2 & chloride, nitrate, nitrite, phosphate, and sulfate \\
\hline 3 & $\begin{array}{c}\mathrm{pH}, \text { Eh, DO, pressure and temperature (field measurements } \\
\text { only) }\end{array}$ \\
\hline 4 & Volatile Organic Compounds (VOCs) \\
\hline 5 & Media in placed Weight and Moisture Content \\
\hline
\end{tabular}


The following tables represent the sampling and analysis regime for the field and laboratory tests identified.

Table 10

Analytical Summary for Field Run \#1

\begin{tabular}{|c|c|c|c|c|c|c|c|}
\hline $\begin{array}{l}\text { Analytical } \\
\text { Group }\end{array}$ & Analytes & $\begin{array}{l}\text { Sample } \\
\text { Bottle }\end{array}$ & $\begin{array}{l}\text { Sample } \\
\text { Volume }\end{array}$ & Preservative & $\begin{array}{l}\text { Analytical } \\
\text { Method }\end{array}$ & Frequency & Location \\
\hline 1 & $\begin{array}{l}\text { RCRA Suite of } \\
\text { Metals: silver, } \\
\text { aluminum, boron, } \\
\text { barium, calcium, } \\
\text { cadmium, cerium, } \\
\text { cobalt, chromium, } \\
\text { copper, iron, } \\
\text { lanthanum, lithium, } \\
\text { magnesium, } \\
\text { manganese, } \\
\text { molybdenum, } \\
\text { sodium, nickel, } \\
\text { phosphorus, lead, } \\
\text { silica, tin, strontium, } \\
\text { titanium, vanadium, } \\
\text { zinc, and zirconium }\end{array}$ & $\begin{array}{l}20 \mathrm{ml} \\
\text { plastic }\end{array}$ & $\begin{array}{l}\text { Fill bottle } \\
20 \mathrm{ml} \\
\text { No } \\
\text { overflow } \\
\text { No } \\
\text { headspace }\end{array}$ & $\begin{array}{l}1 \mathrm{ml} 33.5 \text { to } \\
38 \% \text { reagent } \\
\text { grade } \mathrm{HCl} \\
\text { Filtered }\end{array}$ & ICP-ES & $\begin{array}{l}1,2, \& 4 \\
\text { pore } \\
\text { volumes. } \\
\text { Daily for } \\
\text { remainder } \\
\text { of business } \\
\text { week \#1. } \\
\text { Weekly } \\
\text { thereafter. }\end{array}$ & $\begin{array}{l}\text { Main Inlet, } \\
\text { Outlets to } \\
\text { all } \\
\text { canisters, } \\
\text { All inlets } \\
\text { to Canister } \\
\text { \#2 of each } \\
\text { treatment } \\
\text { train }\end{array}$ \\
\hline 2 & sulfate & $\begin{array}{l}15-20 \mathrm{ml} \\
\text { plastic }\end{array}$ & $15-20 \mathrm{ml}$ & Filtered & IC Anion & $\begin{array}{l}1,2, \& 4 \\
\text { pore } \\
\text { volumes. } \\
\text { Daily for } \\
\text { remainder } \\
\text { of business } \\
\text { week \#1. } \\
\text { Weekly } \\
\text { thereafter }\end{array}$ & $\begin{array}{l}\text { Main Inlet, } \\
\text { Outlets to } \\
\text { all } \\
\text { canisters, } \\
\text { All inlets } \\
\text { to Canister } \\
\# 2 \text { of each } \\
\text { treatment } \\
\text { train }\end{array}$ \\
\hline 3 & $\begin{array}{l}\mathrm{pH}, \mathrm{Eh}, \mathrm{DO} \text {, and } \\
\text { temperature }\end{array}$ & $\begin{array}{l}\text { field } \\
\text { measure- } \\
\text { ment }\end{array}$ & $\begin{array}{l}\text { field } \\
\text { measure- } \\
\text { ment }\end{array}$ & None & Field meter ${ }^{2}$ & $\begin{array}{l}1,2, \& 4 \\
\text { pore } \\
\text { volumes. } \\
\text { Daily for } \\
\text { remainder } \\
\text { of business } \\
\text { week \#1. } \\
\text { Weekly } \\
\text { thereafter }\end{array}$ & $\begin{array}{l}\text { Main Inlet, } \\
\text { Outlets to } \\
\text { all } \\
\text { canisters, } \\
\text { All inlets } \\
\text { to Canister } \\
\# 2 \text { of each } \\
\text { treatment } \\
\text { train }\end{array}$ \\
\hline 4 & VOCs & $\begin{array}{l}22 \mathrm{ml} \\
\text { headspace }\end{array}$ & $7.5 \mathrm{ml}$ & None & GC & $\begin{array}{l}\text { Initial, As } \\
\text { needed }\end{array}$ & Main Inlet \\
\hline 5 & $\begin{array}{l}\text { Moisture Content } \\
\text { in placed weight }\end{array}$ & & & None & $\begin{array}{l}\text { Calibrated } \\
\text { Balance \& } \\
\text { Oven }\end{array}$ & $\begin{array}{l}\text { Pre and } \\
\text { Post Test }\end{array}$ & Canister \\
\hline
\end{tabular}

1 After sample collection, the analytical groups 1,2 , and 4 samples will be refrigerated until analyzed.

$2 \mathrm{pH}, \mathrm{Eh}$, and temperature may be measured with an Oakton $\mathrm{pH} / \mathrm{Eh} /$ temperature meter; $\mathrm{pH}$ and temperature may be measured with

Orion Model 250A; DO may be measured with YSI Model 58; pH, Eh, DO, and temperature may be measured with YSI Model 3800 or 3820 or Hydrolab Model Data Sonde II. 
Table 11

Analytical Summary for Field Run \#2

\begin{tabular}{|c|c|c|c|c|c|c|c|}
\hline $\begin{array}{l}\text { Analytical } \\
\text { Group }\end{array}$ & Analytes & $\begin{array}{l}\text { Sample } \\
\text { Bottle }\end{array}$ & $\begin{array}{l}\text { Sample } \\
\text { Volume }\end{array}$ & Preservative $^{1}$ & $\begin{array}{l}\text { Analytical } \\
\text { Method }\end{array}$ & Frequency & Location \\
\hline 1 & $\begin{array}{l}\text { RCRA Suite of } \\
\text { Metals: silver, } \\
\text { aluminum, boron, } \\
\text { barium, calcium, } \\
\text { cadmium, cerium, } \\
\text { cobalt, chromium, } \\
\text { copper, iron, } \\
\text { lanthanum, } \\
\text { lithium, } \\
\text { magnesium, } \\
\text { manganese, } \\
\text { molybdenum, } \\
\text { sodium, nickel, } \\
\text { phosphorus, lead, } \\
\text { silica, tin, } \\
\text { strontium, } \\
\text { titanium, } \\
\text { vanadium, zinc, } \\
\text { and zirconium }\end{array}$ & $\begin{array}{l}20 \mathrm{ml} \text {. } \\
\text { plastic }\end{array}$ & $\begin{array}{l}\text { Fill bottle } \\
\sim 20 \mathrm{ml} \\
\text { No } \\
\text { overflow } \\
\text { No } \\
\text { headspace }\end{array}$ & $\begin{array}{l}1 \mathrm{ml} 33.5 \text { to } \\
38 \% \text { reagent } \\
\text { grade } \mathrm{HCl} \\
\text { Filtered }\end{array}$ & ICP-ES & $\begin{array}{l}\text { Daily for } \\
\text { remainder } \\
\text { of } \\
\text { business } \\
\text { week \#1. } \\
\text { Weekly } \\
\text { thereafter. }\end{array}$ & $\begin{array}{l}\text { Raw } \\
\text { wastewater, } \\
\text { Potable } \\
\text { water, } \\
\text { Media } \\
\text { solution } \\
\text { from each } \\
\text { train, } \\
\text { Treated } \\
\text { groundwater } \\
\text { from each } \\
\text { train }\end{array}$ \\
\hline 2 & sulfate & $\begin{array}{l}15-20 \mathrm{ml} \\
\text { plastic }\end{array}$ & $15-20 \mathrm{ml}$ & Filtered & IC Anion & $\begin{array}{l}\text { Daily for } \\
\text { remainder } \\
\text { of } \\
\text { business } \\
\text { week \#1. } \\
\text { Weekly } \\
\text { thereafter }\end{array}$ & $\begin{array}{l}\text { Raw } \\
\text { wastewater, } \\
\text { Potable } \\
\text { water, } \\
\text { Media } \\
\text { solution } \\
\text { from each } \\
\text { train, } \\
\text { Treated } \\
\text { groundwater } \\
\text { from each } \\
\text { train }\end{array}$ \\
\hline 3 & $\begin{array}{l}\mathrm{pH}, \mathrm{Eh}, \text { and } \\
\text { temperature }\end{array}$ & $\begin{array}{l}\text { field } \\
\text { measure- } \\
\text { ment }\end{array}$ & $\begin{array}{l}\text { field } \\
\text { measure- } \\
\text { ment }\end{array}$ & None & $\begin{array}{l}\text { Field } \\
\text { meter }^{2}\end{array}$ & $\begin{array}{l}\text { Daily for } \\
\text { remainder } \\
\text { of } \\
\text { business } \\
\text { week \#1. } \\
\text { Weekly } \\
\text { thereafter }\end{array}$ & $\begin{array}{l}\text { Raw } \\
\text { wastewater, } \\
\text { Potable } \\
\text { water, } \\
\text { Media } \\
\text { solution } \\
\text { from each } \\
\text { train, } \\
\text { Treated } \\
\text { groundwater } \\
\text { from each } \\
\text { train }\end{array}$ \\
\hline
\end{tabular}


Table 12

Analytical Summary for Field Run \#3

\begin{tabular}{|c|c|c|c|c|c|c|c|}
\hline $\begin{array}{l}\text { Analytical } \\
\text { Group }\end{array}$ & Analytes & $\begin{array}{l}\text { Sample } \\
\text { Bottle }\end{array}$ & $\begin{array}{l}\text { Sample } \\
\text { Volume }\end{array}$ & Preservative $^{1}$ & $\begin{array}{l}\text { Analytical } \\
\text { Method }\end{array}$ & Frequency & Location \\
\hline 1 & $\begin{array}{l}\text { RCRA Suite of } \\
\text { Metals: silver, } \\
\text { aluminum, boron, } \\
\text { barium, calcium, } \\
\text { cadmium, cerium, } \\
\text { cobalt, chromium, } \\
\text { copper, iron, } \\
\text { lanthanum, } \\
\text { lithium, } \\
\text { magnesium, } \\
\text { manganese, } \\
\text { molybdenum, } \\
\text { sodium, nickel, } \\
\text { phosphorus, lead, } \\
\text { silica, tin, } \\
\text { strontium, } \\
\text { titanium, } \\
\text { vanadium, zinc, } \\
\text { and zirconium }\end{array}$ & $\begin{array}{l}20 \mathrm{ml} \\
\text { plastic }\end{array}$ & $\begin{array}{l}\text { Fill bottle } \\
-20 \mathrm{ml} \\
\text { No } \\
\text { overflow } \\
\text { No } \\
\text { headspace }\end{array}$ & $\begin{array}{l}1 \mathrm{ml} 33.5 \text { to } \\
38 \% \text { reagent } \\
\text { grade } \mathrm{HCl} \\
\text { Filtered }\end{array}$ & ICP-ES & $\begin{array}{l}\text { Daily for } \\
\text { remainder } \\
\text { of } \\
\text { business } \\
\text { week \#1. } \\
\text { Weekly } \\
\text { thereafter. }\end{array}$ & $\begin{array}{l}\text { Inlet } \\
\text { phosphate } \\
\text { solution, } \\
\text { Raw } \\
\text { wastewater, } \\
\text { Outlets of } \\
\text { each } \\
\text { treatment } \\
\text { train }\end{array}$ \\
\hline 2 & $\begin{array}{l}\text { phosphate and } \\
\text { sulfate }\end{array}$ & $\begin{array}{l}15-20 \mathrm{ml} \\
\text { plastic }\end{array}$ & $15-20 \mathrm{ml}$ & Filtered & IC Anion & $\begin{array}{l}\text { Daily for } \\
\text { remainder } \\
\text { of } \\
\text { business } \\
\text { week \#1. } \\
\text { Weekly } \\
\text { thereafter }\end{array}$ & $\begin{array}{l}\text { Inlet } \\
\text { phosphate } \\
\text { solution, } \\
\text { Raw } \\
\text { wastewater, } \\
\text { Outlets of } \\
\text { each } \\
\text { treatment } \\
\text { train }\end{array}$ \\
\hline 3 & $\begin{array}{l}\mathrm{pH}, \text { Eh, and } \\
\text { temperature }\end{array}$ & $\begin{array}{l}\text { field } \\
\text { measure- } \\
\text { ment }\end{array}$ & $\begin{array}{l}\text { field } \\
\text { measure- } \\
\text { ment }\end{array}$ & None & $\begin{array}{l}\text { Field } \\
\text { meter }^{2}\end{array}$ & $\begin{array}{l}\text { Daily for } \\
\text { remainder } \\
\text { of } \\
\text { business } \\
\text { week \#1. } \\
\text { Weekly } \\
\text { thereafter }\end{array}$ & $\begin{array}{l}\text { Inlet } \\
\text { phosphate } \\
\text { solution, } \\
\text { Raw } \\
\text { wastewater, } \\
\text { Outlets of } \\
\text { each } \\
\text { treatment } \\
\text { train }\end{array}$ \\
\hline
\end{tabular}


Table 13

Analytical Summary for Lab Test \#1

\begin{tabular}{|c|c|c|c|c|c|c|}
\hline $\begin{array}{l}\text { Analytical } \\
\text { Group }\end{array}$ & Analytes & $\begin{array}{l}\text { Sample } \\
\text { Bottle }\end{array}$ & $\begin{array}{l}\text { Sample } \\
\text { Volume }\end{array}$ & Preservative $^{1}$ & $\begin{array}{l}\text { Analytical. } \\
\text { Method }\end{array}$ & Location \\
\hline 1 & $\begin{array}{l}\text { RCRA Suite of } \\
\text { Metals: silver, } \\
\text { aluminum, boron, } \\
\text { barium, calcium, } \\
\text { cadmium, cerium, } \\
\text { cobalt, chromium, } \\
\text { copper, iron, } \\
\text { lanthanum, } \\
\text { lithium, } \\
\text { magnesium, } \\
\text { manganese, } \\
\text { molybdenum, } \\
\text { sodium, nickel, } \\
\text { phosphorus, lead, } \\
\text { silica, tin, } \\
\text { strontium, } \\
\text { titanium, } \\
\text { vanadium, zinc, } \\
\text { and zirconium }\end{array}$ & $20 \mathrm{ml}$ plastic & $\begin{array}{l}\text { Fill bottle } \\
-20 \mathrm{ml} \\
\text { No overflow } \\
\text { No headspace }\end{array}$ & $\begin{array}{l}1 \mathrm{ml} 33.5 \text { to } \\
38 \% \text { reagent } \\
\text { grade } \mathrm{HCl} \\
\text { Filtered }\end{array}$ & ICP-ES & $\begin{array}{l}\text { Nanopure } \\
\text { water, } \\
\text { Adjusted } \\
\text { Nanopure } \\
\text { water, } \\
\text { Phosphate } \\
\text { rock solutions } \\
\text { at pH=4.5, } \\
\text { Strongest } \\
\text { potassium } \\
\text { phosphate, } \\
\text { and sodium } \\
\text { phosphate } \\
\text { solutions, } \\
\text { Contaminated } \\
\text { groundwater, } \\
\text { Treated } \\
\text { groundwater }\end{array}$ \\
\hline 2 & $\begin{array}{l}\text { phosphate, and } \\
\text { sulfate }\end{array}$ & $15-20 \mathrm{ml}$ plastic & $15-20 \mathrm{ml}$ & Filtered & IC Anion & $\begin{array}{l}\text { Nanopure } \\
\text { water, } \\
\text { Adjusted } \\
\text { Nanopure } \\
\text { water, } \\
\text { Treatment } \\
\text { media } \\
\text { solutions, } \\
\text { Contaminated } \\
\text { groundwater, } \\
\text { Treated } \\
\text { groundwater }\end{array}$ \\
\hline 3 & $\mathrm{pH}, \mathrm{Eh}$ & $\begin{array}{l}50 \mathrm{ml} \text { centrifuge } \\
\text { tube } \\
\text { lab measure- } \\
\text { ment }\end{array}$ & $\begin{array}{l}50 \mathrm{ml} \text { centrifuge } \\
\text { tube } \\
\text { lab measure- } \\
\text { ment }\end{array}$ & None & $\begin{array}{l}\text { Field } \\
\text { meter }^{2}\end{array}$ & $\begin{array}{l}\text { Nanopure } \\
\text { water, } \\
\text { Adjusted } \\
\text { Nanopure } \\
\text { water, } \\
\text { Treatment } \\
\text { media } \\
\text { solutions, } \\
\text { Contaminated } \\
\text { groundwater, } \\
\text { Treated } \\
\text { groundwater }\end{array}$ \\
\hline
\end{tabular}


Table 14

Analytical Summary for Laboratory Test \#2

\begin{tabular}{|c|c|c|c|c|c|c|}
\hline $\begin{array}{l}\text { Analytical } \\
\text { Group }\end{array}$ & Analytes & $\begin{array}{l}\text { Sample } \\
\text { Bottle }\end{array}$ & $\begin{array}{l}\text { Sample } \\
\text { Volume }\end{array}$ & Preservative $^{1}$ & $\begin{array}{l}\text { Analytical } \\
\text { Method }\end{array}$ & Location \\
\hline 1 & $\begin{array}{l}\text { RCRA Suite of } \\
\text { Metals: silver, } \\
\text { aluminum, boron, } \\
\text { barium, calcium, } \\
\text { cadmium, cerium, } \\
\text { cobalt, chromium, } \\
\text { copper, iron, } \\
\text { lanthanum, } \\
\text { lithium, } \\
\text { magnesium, } \\
\text { manganese, } \\
\text { molybdenum, } \\
\text { sodium, nickel, } \\
\text { phosphorus, lead, } \\
\text { silica, tin, } \\
\text { strontium, } \\
\text { titanium, } \\
\text { vanadium, zinc, } \\
\text { and zirconium }\end{array}$ & $20 \mathrm{ml}$ plastic & $\begin{array}{l}\text { Fill bottle } \\
-20 \mathrm{ml} \\
\text { No overflow } \\
\text { No headspace }\end{array}$ & $\begin{array}{l}1 \mathrm{ml} 33.5 \text { to } \\
38 \% \text { reagent } \\
\text { grade } \mathrm{HCl} \\
\text { Filtered }\end{array}$ & ICP-ES & $\begin{array}{l}\text { Treatment } \\
\text { media } \\
\text { solutions, } \\
\text { Treated } \\
\text { groundwater }\end{array}$ \\
\hline 2 & $\begin{array}{l}\text { phosphate, and } \\
\text { sulfate }\end{array}$ & $15-20 \mathrm{ml}$ plastic & $15-20 \mathrm{ml}$ & Filtered & IC Anion & $\begin{array}{l}\text { Treatment } \\
\text { media } \\
\text { solutions, } \\
\text { Treated } \\
\text { groundwater }\end{array}$ \\
\hline 3 & $\mathrm{pH}, \mathrm{Eh}$ & $\begin{array}{l}50 \mathrm{ml} \text { centrifuge } \\
\text { tube } \\
\text { lab measure- } \\
\text { ment }\end{array}$ & $\begin{array}{l}50 \mathrm{ml} \text { centrifuge } \\
\text { tube } \\
\text { lab measure- } \\
\text { ment }\end{array}$ & None & $\begin{array}{l}\text { Field } \\
\text { meter }^{2}\end{array}$ & $\begin{array}{l}\text { Treatment } \\
\text { media } \\
\text { solutions, } \\
\text { Treated } \\
\text { groundwater }\end{array}$ \\
\hline
\end{tabular}


Table 15

Analytical Summary for Laboratory Test \#3

\begin{tabular}{|c|c|c|c|c|c|c|}
\hline $\begin{array}{l}\text { Analytical } \\
\text { Group }\end{array}$ & Analytes & $\begin{array}{l}\text { Sample } \\
\text { Bottle }\end{array}$ & $\begin{array}{l}\text { Sample } \\
\text { Volume }\end{array}$ & Preservative $^{1}$ & $\begin{array}{l}\text { Analytical } \\
\text { Method }\end{array}$ & Location \\
\hline 1 & $\begin{array}{l}\text { RCRA Suite of Metals: } \\
\text { silver, aluminum, boron, } \\
\text { barium, calcium, cadmium, } \\
\text { cerium, cobalt, chromium, } \\
\text { copper, iron, lanthanum, } \\
\text { lithium, magnesium, } \\
\text { manganese, molybdenum, } \\
\text { sodium, nickel, } \\
\text { phosphorus, lead, silica, } \\
\text { tin, strontium, titanium, } \\
\text { vanadium, zinc, and } \\
\text { żirconium }\end{array}$ & $20 \mathrm{ml}$ plastic & $\begin{array}{l}\text { Fill bottle } \\
\sim 20 \mathrm{ml} \\
\text { No overflow } \\
\text { No headspace }\end{array}$ & $\begin{array}{l}1 \mathrm{ml} 33.5 \text { to } \\
38 \% \text { reagent } \\
\text { grade } \mathrm{HCl} \\
\text { Filtered }\end{array}$ & ICP-ES & $\begin{array}{l}\text { Contaminated } \\
\text { groundwater, } \\
\text { Treated } \\
\text { groundwater }\end{array}$ \\
\hline 2 & phosphate and sulfate & $\begin{array}{l}15-20 \mathrm{ml} \\
\text { plastic }\end{array}$ & $15-20 \mathrm{ml}$ & Filtered & IC Anion & $\begin{array}{l}\text { Contaminated } \\
\text { groundwater, } \\
\text { Treated } \\
\text { groundwater }\end{array}$ \\
\hline 3 & $\mathrm{pH}, \mathrm{Eh}$ & $\begin{array}{l}50 \mathrm{ml} \\
\text { centrifuge tube } \\
\text { lab measure- } \\
\text { ment }\end{array}$ & $\begin{array}{l}50 \mathrm{ml} \\
\text { centrifuge tube } \\
\text { lab measure- } \\
\text { ment }\end{array}$ & None & $\begin{array}{l}\text { Field } \\
\text { meter }^{2}\end{array}$ & $\begin{array}{l}\text { Contaminated } \\
\text { groundwater, } \\
\text { Treated } \\
\text { groundwater }\end{array}$ \\
\hline
\end{tabular}

Table 16

Analytical Summary for Laboratory Test \#4

\begin{tabular}{||l|l|l|l|l|l|l|}
\hline \hline $\begin{array}{l}\text { Analytical } \\
\text { Group }\end{array}$ & Analytes & $\begin{array}{l}\text { Sample } \\
\text { Bottle } \\
\text { Volume }\end{array}$ & Preservative & $\begin{array}{l}\text { Analytical } \\
\text { Method }\end{array}$ & Location \\
\hline 1 & $\begin{array}{l}\text { RCRA Suite of Metals: } \\
\text { silver, aluminum, boron, } \\
\text { barium, calcium, cadmium, } \\
\text { cerium, cobalt, chromium, } \\
\text { copper, iron, lanthanum, } \\
\text { lithium, magnesium, } \\
\text { manganese, molybdenum, } \\
\text { sodium, nickel, } \\
\text { phosphorus, lead, silica, } \\
\text { tin, strontium, titanium, } \\
\text { vanadium, zinc, and } \\
\text { zirconium }\end{array}$ & $20 \mathrm{ml}$ plastic & $\begin{array}{l}\text { Fill bottle } \\
\sim 20 \mathrm{ml} \\
\text { No overflow }\end{array}$ & $\begin{array}{l}1 \mathrm{ml} 33.5 \text { to } \\
38 \% \text { reagent } \\
\text { grade HCl }\end{array}$ & $\begin{array}{l}\text { ICP-ES } \\
\text { No headspace }\end{array}$ & $\begin{array}{l}\text { Fontaminated } \\
\text { groundwater, } \\
\text { Aerated } \\
\text { groundwater, } \\
\text { Treatment } \\
\text { media } \\
\text { solution } \\
\text { Treated } \\
\text { groundwater }\end{array}$ \\
\hline 2 & phosphate and sulfate & $\begin{array}{l}15-20 \mathrm{ml} \\
\text { plastic }\end{array}$ & $15-20 \mathrm{ml}$ & Filtered & IC Anion & $\begin{array}{l}\text { Contaminated } \\
\text { groundwater, } \\
\text { Treated } \\
\text { groundwater }\end{array}$ \\
\hline
\end{tabular}




\subsection{Quality Control}

The following quality control measures were utilized during the demonstration:

- Samples received analytical quality control per ADS standard procedures.

- One duplicate and one control sample were taken each week and analyzed for ion analysis.

- Photographs were taken of the media before and after use.

\subsection{Deviations}

Duplicates and control samples were not pulled for ion analysis during Field Run \#3. An independent laboratory was used for a duplicate sample in Field Run \#3.

Dissolve oxygen was only measured for Field Run \#1. No instrumentation was available for subsequent runs.

Moisture content was not performed for Field Runs $2 \& 3$. Material denatured during run \#1, resulting in erroneous outcomes.

Samples were allowed to settle for 72 hours instead of 24 hours in Laboratory Test \#3.

Photographs were not taken of the media before and after use. Representative samples of the media were collected and stored.

\section{RESULTS AND DISCUSSION}

\section{$5.1 \quad$ Field Run \#1}

\subsubsection{Initial Design Concept Prior to Media Studies}

Figure 5 shows the original concept that was to be implemented for the project. This initial conceptual design configuration consisted of an interceptor trench, a treatment cell and a redistribution system. The interceptor trench consisted of a perforated pipe in a gravel bed for the collection of the contaminated groundwater and transport to the treatment cell. The gravel bed was designed so that it may contain permeable treatment media determined in the ex situ treatability study to provide an initial treatment prior to the treatment cell itself. The treatment cell consisted of a flow through, removable, treatment media canister. The existing D-Area Interceptor Well, DIW-1 would be utilized as the redistribution system. The treatment cell would be directly connected to the barrier system through a pipeline to one of the existing 6 inch stainless steel screens on the system so that an integrated groundwater interception, treatment, and redistribution system was produced. 


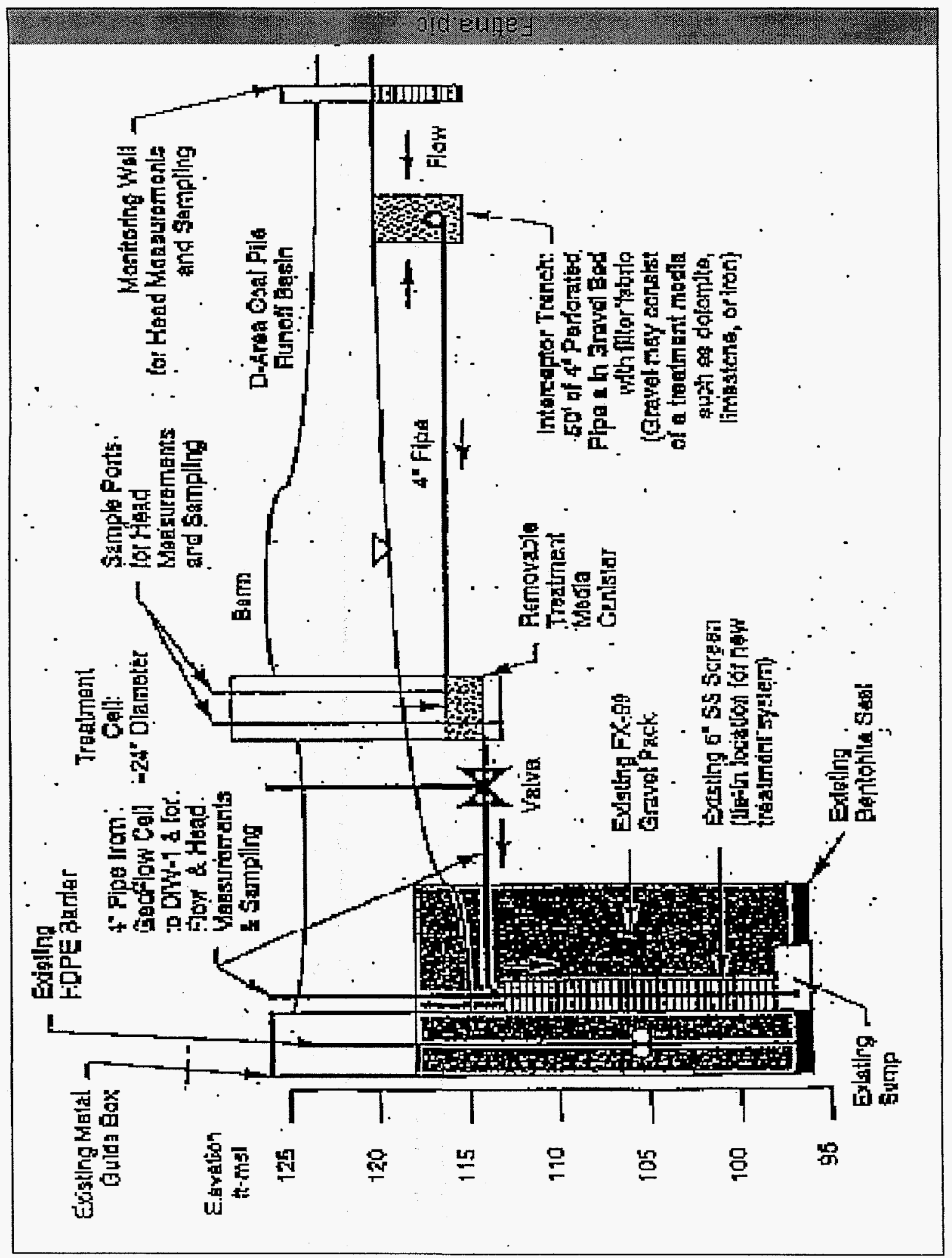

Figure 5 - D-Area Treatment System Original Conceptual Design 


\subsubsection{Field Observations Field Run 1}

\subsubsection{Pressures/Plugging}

During Field Run 1, plugging and pressure problems were noted. The pressures ranged from -2.53 psig at the down gradient discharge point to $15.7 \mathrm{psig}$. Precipitates formed in the canisters as well as the lines and 15-gallon settling tanks. Most of the precipitates were held up in the media and filter fabric. The filter fabric was placed directly in contact with the bottom of the canister to allow for an even distribution of the influent to the canister. Due to the volume of precipitates, plugging at the inlets and outlets to canisters caused increases in pressures to $15 \mathrm{psig}$, the design limit of the system, in some cases. The $3 / 8$ " polyethylene tubing was periodically removed from the bottom of the media canisters and the inlet cleared (gently scraped with a nonmetallic object and rinsed with deionized water) on all four trains. The filter fabric was also replaced at the top of Train 1 Canister 1 on two occasions due to excessive precipitate build up. Cementing of the treatment media occurred in Train 3-Canister 2 and Train 4-Canister 1 which also contributed to the plugging and pressure difficulties. These difficulties could not be corrected during the run. This was consistent with the system pressures and observations noted in the field.

\subsubsection{Media}

The following field observations were noted after the run when the canisters were examined:

Train 1 Canister 1-Limestone

Inlet-The inlet to the canister looked similar to the outlet except for the filter fabric had a brown, white, and gray discoloration. This might have been a white precipitate, which was discolored orange. Outlet-A small amount of gray-white paste was found on the filter fabric at the outlet of the limestone. The paste was somewhat coating the limestone media.

Train 1 Canister 2-Peat Inlet-Some gray-white paste was in the first foot of the peat canister. The down stream side of the filter fabric showed no obvious signs of change. The upstream side of the fabric had a small amount of gray-white paste with a small amount of orange. This was much less paste than in the second canisters of the other treatment trains associated with the limestone.

Outlet-The filter fabric and media showed no obvious signs of change. 
Train 2 Canister 1-Limestone

Inlet-The inlet to the canister had a little more orange precipitate on the limestone itself along with the white precipitate found in the other canisters. There also appeared to be more paste on the down stream side of the filter fabric than in the other canisters. Also a similar brown, white, and gray discoloration on the upstream side of the filter fabric was noted. This was slightly more than seen in Canister 1 Train 1.

Outlet-A small amount of gray-white paste found on the filter fabric at the outlet of the limestone. A paste was somewhat coating the limestone.

Train 2 Canister 2-Zeolite

Inlet-The down streamside of the filter fabric and into the zeolite showed no obvious signs of change and the zeolite media was loose. The upstream side of the filter fabric had a gray-white paste with an orange layer in it (approximately $1 / 8$ " thickness). Outlet-Showed no obvious signs of change and the material was still loose.

Train 3 Canister 1-Limestone Inlet-The inlet had more brown orange precipitate than either Train 1 or 2 limestone. The down streamside of the filter fabric was more orange/brown than seen in the other limestone. The upstream side was similarly discolored as the downstream side of the filter fabric but even more so. There was an inner ring of brown and an outer ring of reddish brown on down streamside of filter fabric. Outlet-Appeared to have even less paste than Train 1 and 2 limestone canisters but still a significant amount.

Train3 Canister 2-Granular Cast Iron Inlet-Was cemented together with a gray white precipitate on the upstream side of the filter fabric. A white precipitate paste approximately $1 / 4$ " thick was in the center of the cap and thinner at the edges. The white precipitate appeared to have an orange layer of precipitate in it. There was some spotty orange staining of the filter fabric on the down streamside. A white precipitate extended into the pores.

Outlet-Showed no obvious signs of change and no obvious precipitates material fairly loose iron still black similar to unused material. Filter fabric was stained with rust color but no significant precipitation was noted. 
Train 4 Canister 1-Phosphate Rock

Inlet-There appeared to be precipitate nodules without the granular phosphate rock in it and appeared to be cemented phosphate nodules in the inlet of the canister. The cemented nodules could be broken by hand but had a definite shape when removed from the surrounding material. There was an approximately one foot long section of cemented phosphate rock at the bottom of the canister. The plug started at approximately 6" from the bottom. Additional cemented sections were found in the first $2.5^{\prime}$ of the canister. Outlet-Showed no obvious signs of change and no obvious precipitate material fairly loose.

Train 4 Canister 2-Granular Cast Iron

Inlet-Had a thin greenish precipitate on the upstream side of the filter fabric. The downstream side of the filter fabric had a rusty discoloration. The iron was cemented and had some greenish precipitate within the pore spaces. The cemented iron was not as hard as the cemented iron that from Train 3 and was less cemented. Outlet -The media had a green and orange-brown staining and the water had a greenish tint. The upstream side had an orange-brown staining on the filter fabric. The downstream side had mostly green staining with an orange-brown staining in the center.

\subsubsection{Data Observations Field Run 1}

\subsubsection{Flow Summary}

The following table shows the average flows for each treatment train during this field run:

\begin{tabular}{||c|c|c|}
\hline Train & $\begin{array}{c}\text { Day 1 } \\
\mathrm{ml} / \mathrm{min}\end{array}$ & $\begin{array}{c}\text { Remainder of Test } \\
\mathrm{ml} / \mathrm{min}\end{array}$ \\
\hline 1 & 46 & 102 \\
\hline 2 & 30 & 100 \\
\hline 3 & 60 & 118 \\
\hline 4 & 48 & 137 \\
\hline
\end{tabular}

\subsubsection{Analytical Summary}

Figures 6-17 depict the primary contaminants (aluminum, chromium, iron, and nickel) through the canisters during this campaign. Preliminary observations are summarized below and shown in Tables 17-22. 


\section{Limestone Canisters (Trains 1,2 \& 3, Canister 1)}

There was significant aluminum and chromium removal throughout test. Outlet concentrations of aluminum and chromium ranged from less than detection to some value significantly less than the inlet concentration over time. There was little iron and nickel removal except initially. The initial outlet concentrations of iron and nickel were however above the drinking water limits. It appears that iron from the groundwater may have been released from the canisters toward the end of the test. There was some silica reduction throughout the test. Significant calcium was released from the limestone but the levels decreased to lower values over the course of the campaign. There was some magnesium released from the limestone initially but the release decreased over time to a resulting concentration similar to the inlet concentration. There was some slight reduction in sulfate throughout the test. The $\mathrm{pH}$ increased throughout the test with percentage increases decreasing over time. The dissolved oxygen concentrations also showed percentage increases decreasing over time to nearly zero percent. The Eh reduction decreased over time to nearly zero percent during the campaign. It was noted that the limestone had developed a coating over time.

\section{Phosphate Rock (Train 4 Canister 1)}

The increase in the outlet phosphorus concentration decreased over time. There was significant aluminum and chromium removal throughout test. Outlet aluminum concentrations ranged from less than detection to $24 \mathrm{ppm}$. This was significantly less than the inlet concentration (95-179 ppm).There was significant iron and nickel removal initially. The outlet nickel concentration went from less than detect to essentially no treatment during the test. The iron outlet concentration from the groundwater ranged from less than detect to greater than inlet concentration. This suggests iron from the groundwater may have been redissolving during the test. Significant calcium was released from the phosphate rock but the levels decreased to lower values over the course of the campaign. There was some magnesium released from the limestone initially but that decreased over time to essentially minimal release. There was an initial reduction in manganese concentrations followed by a slight possible increase towards the end of the campaign. There was some slight reduction in sulfate throughout the test. The $\mathrm{pH}$ increased throughout the test with percentage increases decreasing over time. The dissolved oxygen concentrations also increased with percentage increases decreasing over time to zero percent. The Eh reduction decreased over time to zero percent during the campaign. It was noted that the phosphate rock had cemented over time. 
Peat (Train 1 Canister 2)

The peat canister followed the limestone canister; therefore initially the peat had very little input of aluminum and chromium. Once the aluminum and chromium started breaking through the limestone canister, significant aluminum and chromium treatment was provided throughout the test. There was significant iron and nickel removal initially. The outlet concentrations ranged from slightly above the detection limit; to possibly being exchanged by chromium and aluminum (i.e. greater than inlet) when the limestone canister started releasing more chromium and aluminum in its effluent. Towards the end of the test, there was essentially no treatment observed for iron and nickel. There was some manganese removal initially followed by displacement by aluminum. There was also some slight silica removal throughout the test. The $\mathrm{pH}$ decreased from average of 5.2 to 3.6 with the magnitude of decreases decreasing with time. The dissolved oxygen appeared initially increased followed by essentially no change from inlet. The Eh increased from average 314 to 558 with the magnitude of increases decreasing with time. Once the canisters were inspected, it was noted that the peat appeared unchanged.

\section{Zeolite (Train 2 Canister 2)}

The zeolite canister followed the limestone canister; therefore initially the zeolite had very little input of aluminum and chromium. Once the aluminum and chromium started breaking through the limestone canister, some aluminum and chromium treatment was provided by the zeolite upon initial limestone canister break through. The aluminum and chromium removal then went to essentially no treatment. There was significant iron and nickel removal initially. The outlet concentrations of iron and nickel ranged from below the detection limit to essentially no treatment. It does not appear however that the aluminum and chromium displaced iron and nickel as in the case of the peat. There was some manganese removal initially followed by displacement by aluminum. There was also some slight silica removal throughout the test. The $\mathrm{pH}$ remained fairly constant throughout the test. The dissolved oxygen increased through the zeolite from average of 3.14 to 6.08 . The zeolite canister initially had significant increases in Eh followed by lesser increases over time. Once the canisters were inspected, it was noted that the zeolite appeared unchanged. 


\section{Iron Canister Following Limestone (Train 3 Canister 2)}

This iron canister followed the limestone canister. Aluminum and chromium had significant treatment throughout the test. There was significant nickel removal initially. The outlet concentrations of nickel ranged from below the detection limit to essentially no treatment. There was significant iron addition throughout the test. Some slight manganese addition throughout the test was observed as well as some silica removal throughout the test. There was some slight sulfate removal throughout the test. The $\mathrm{pH}$ increases were fairly constant over time. The dissolved oxygen decreases reduced over time to essentially no decrease. The Eh reduction remained fairly constant over time. It was noted that the iron had cemented over time.

Iron Canister Following Phosphate Rock (Train 4 Canister 2) This iron canister followed the phosphate rock canister; therefore initially the iron had very little input of chromium. There was significant aluminum and chromium treatment throughout the test. The inlet concentrations of aluminum and chromium increased over time due to decreased removal in the phosphate rock canister. There was significant nickel removal initially. The outlet nickel concentrations ranged from less than detect to essentially no treatment. There was significant iron addition throughout the test. Some slight manganese addition throughout the test was observed as well as some silica removal throughout the test. There was some slight sulfate removal throughout the test. The $\mathrm{pH}$ increases were fairly constant over time. The dissolved oxygen decreases variable throughout the campaign. The Eh reduction remained fairly constant over time. It was noted that the iron had cemented over time. 
Figure 6. Ex-Situ Treatment Media Evaluation Run \#1 Contact Limestone Media

(Representative of Trains 1,2 \&3 Canister 1)

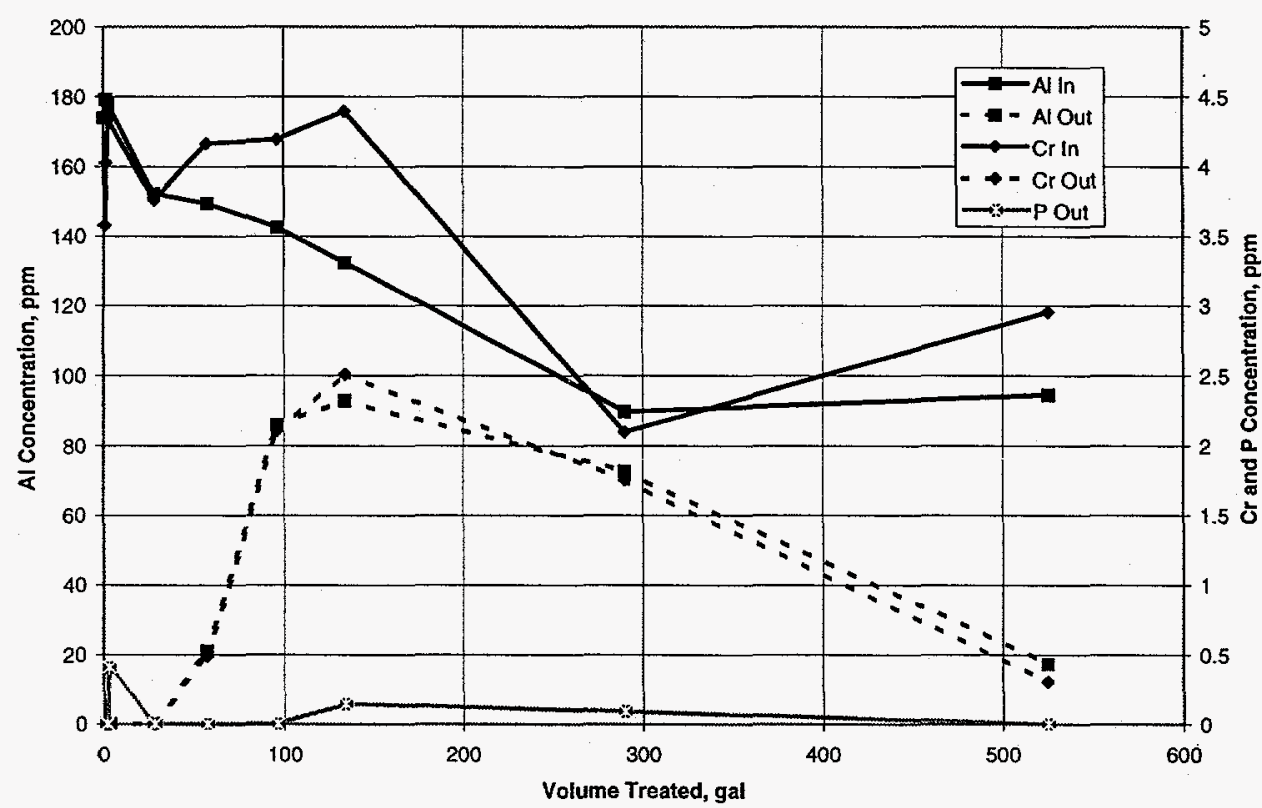

Figure 7. Ex-Situ Treatment Media Evaluation Run \#1 Contact Limestone Media (Representative of Trains 1,2 \&3 Canister 1)

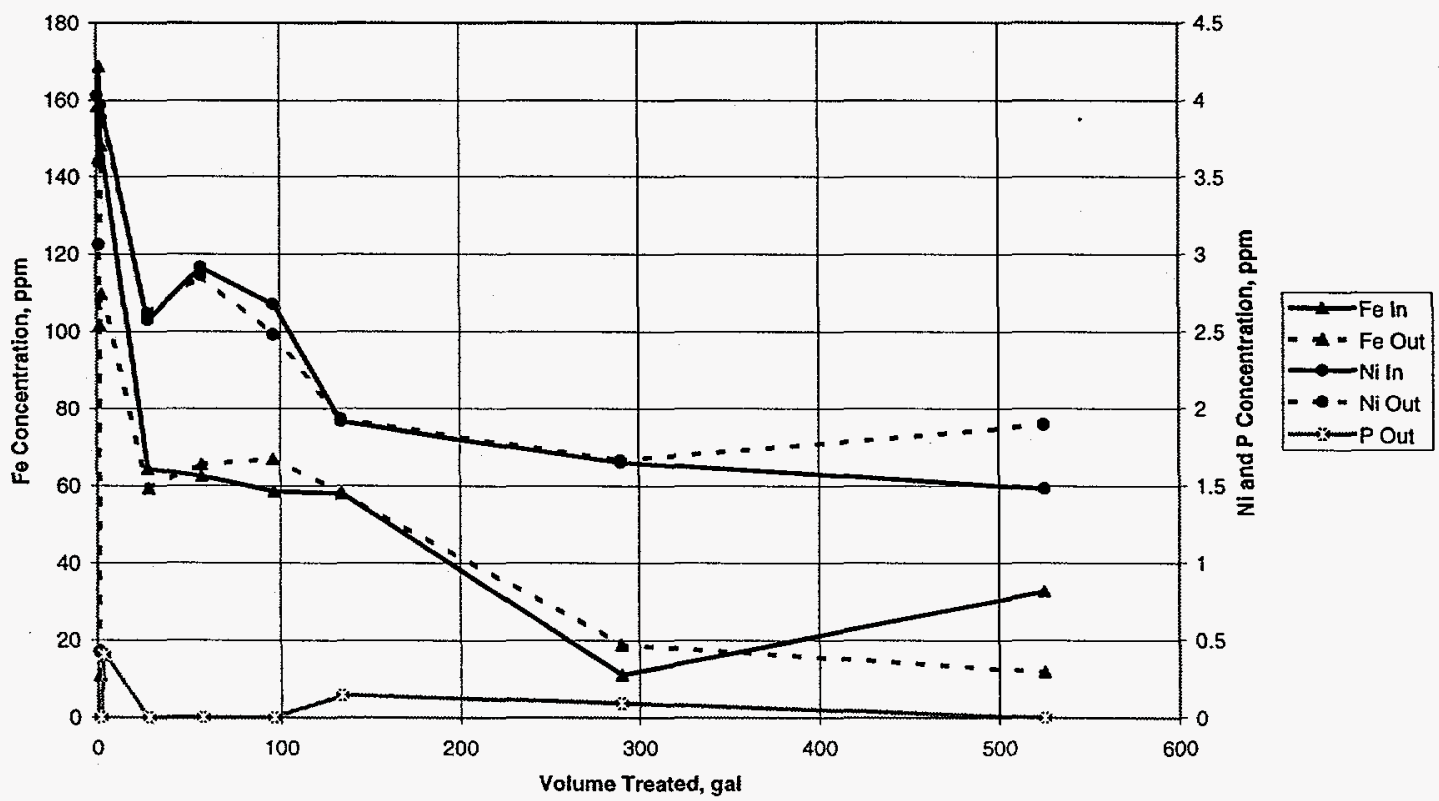


Figure 8. Ex-Situ Treatment Media Evaluation Run \#1 Contact Phosphate Media

(Train 4 Canister 1 )

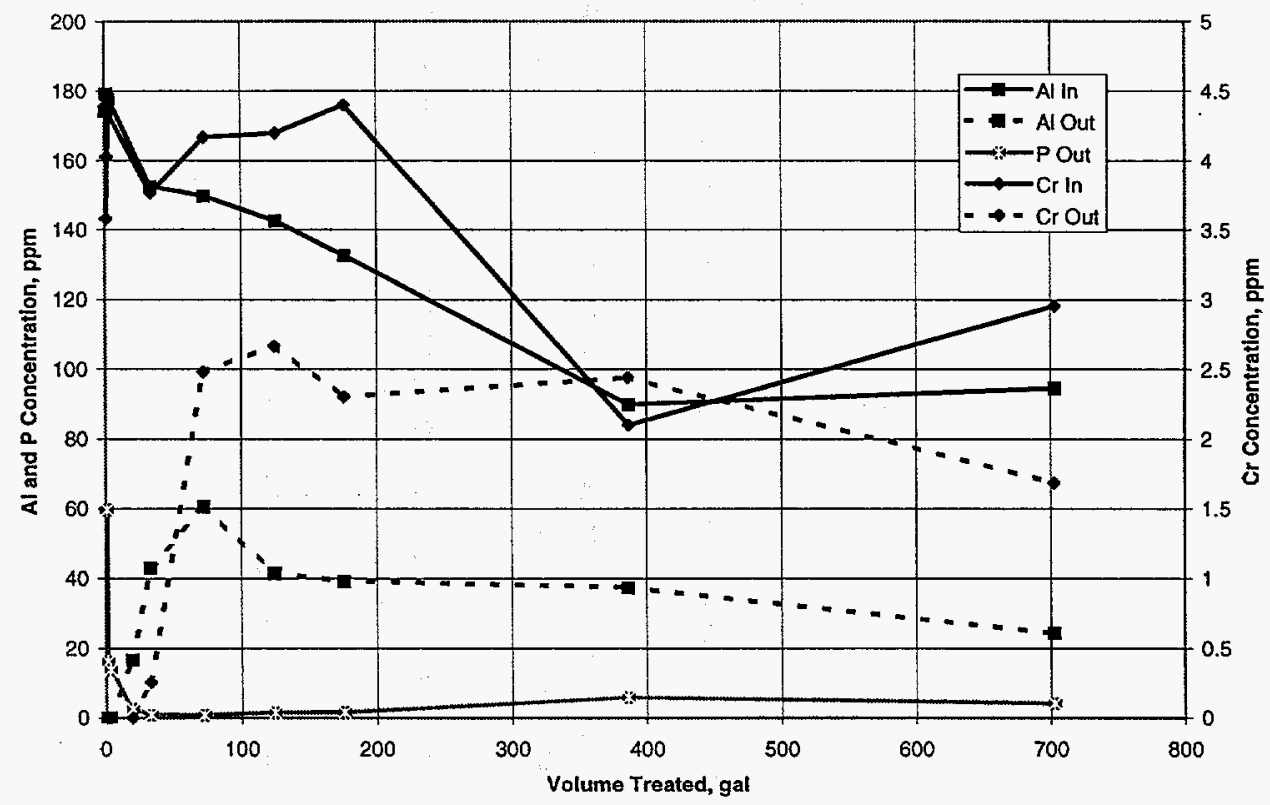

Figure 9. Ex-Situ Treatment Media Evaluation Run \#1 Contact Phosphate Media

(Train 4 Canister 1 )

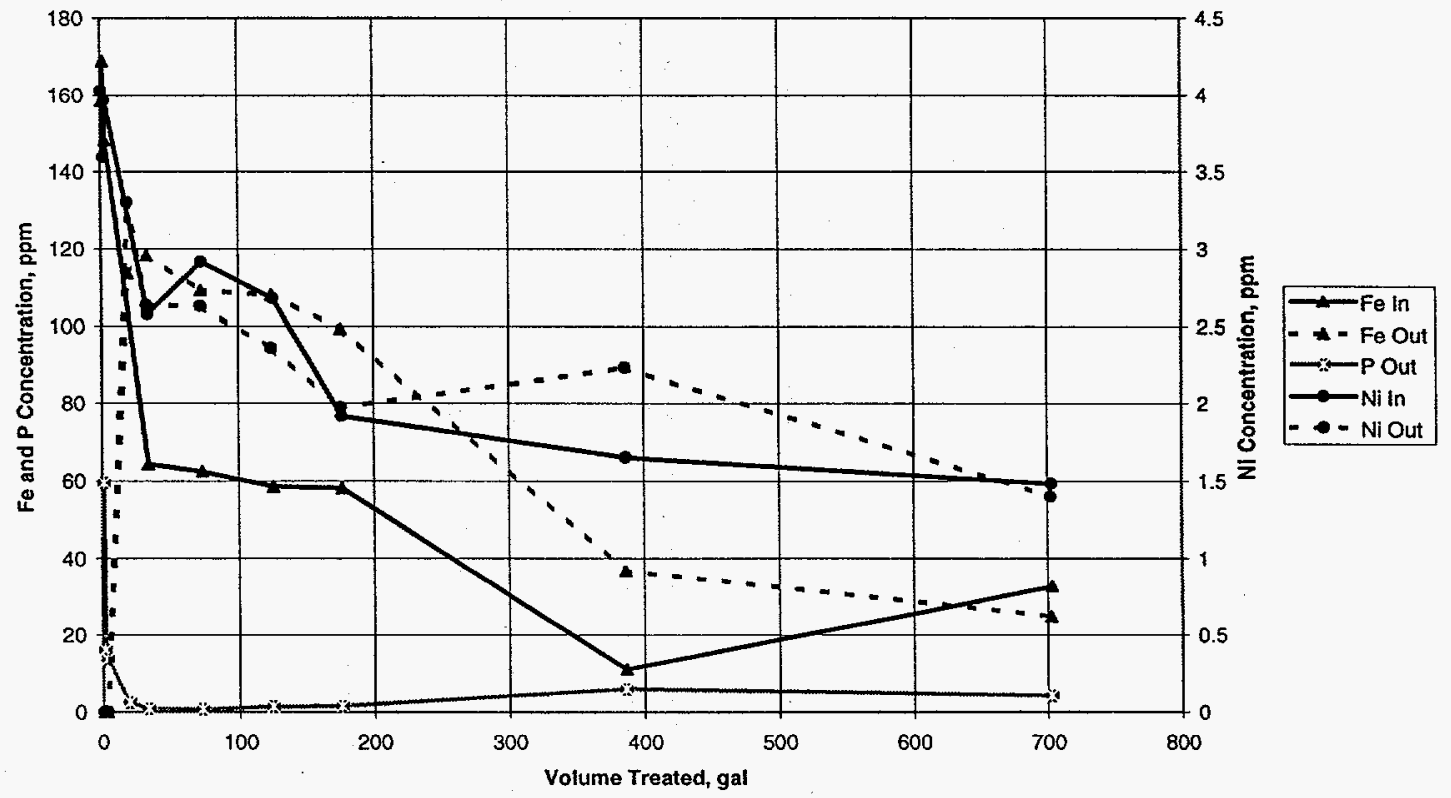


Figure 10. Ex-Situ Treatment Media Evaluation Run \#1

Contact Peat Media

(Train 1 Canister 2)

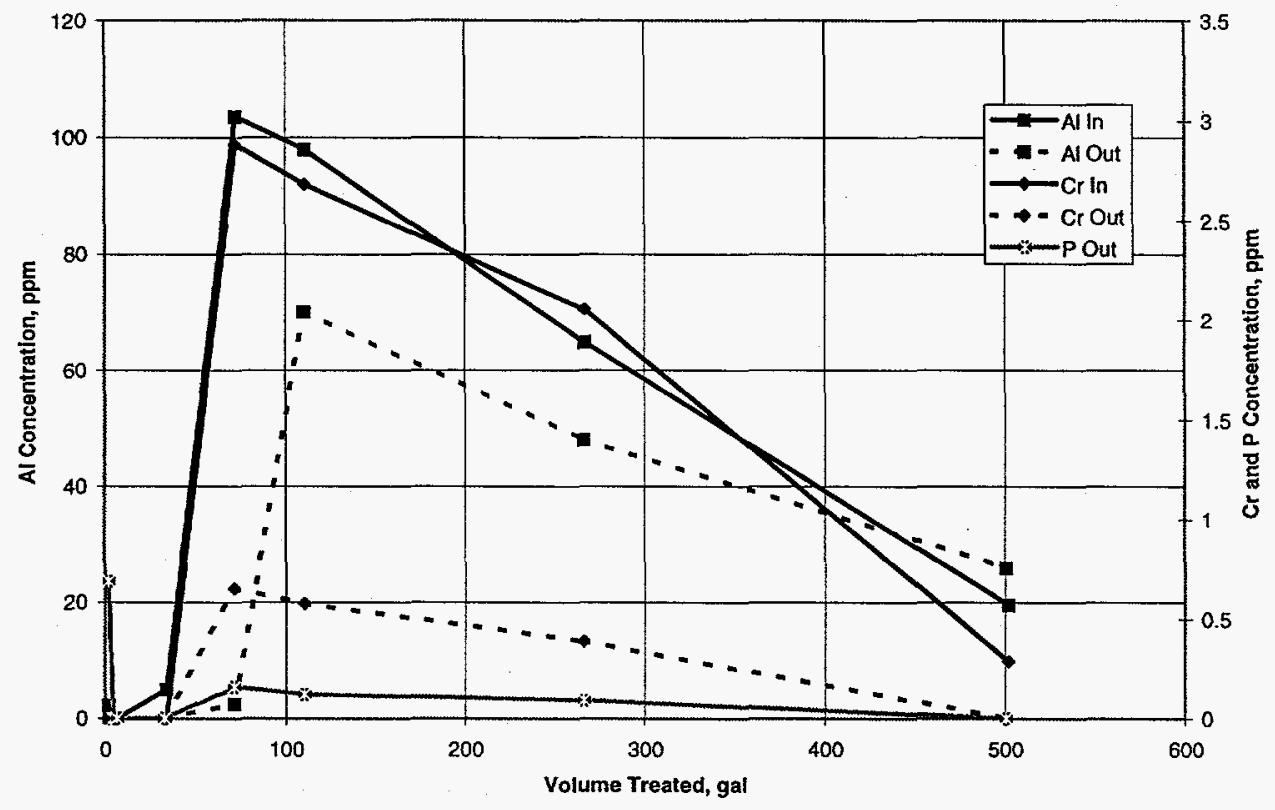

Figure 11. Ex-Situ Treatment Media Evaluation Run \#1 Contact Peat Media

(Train 1 Canister 2)

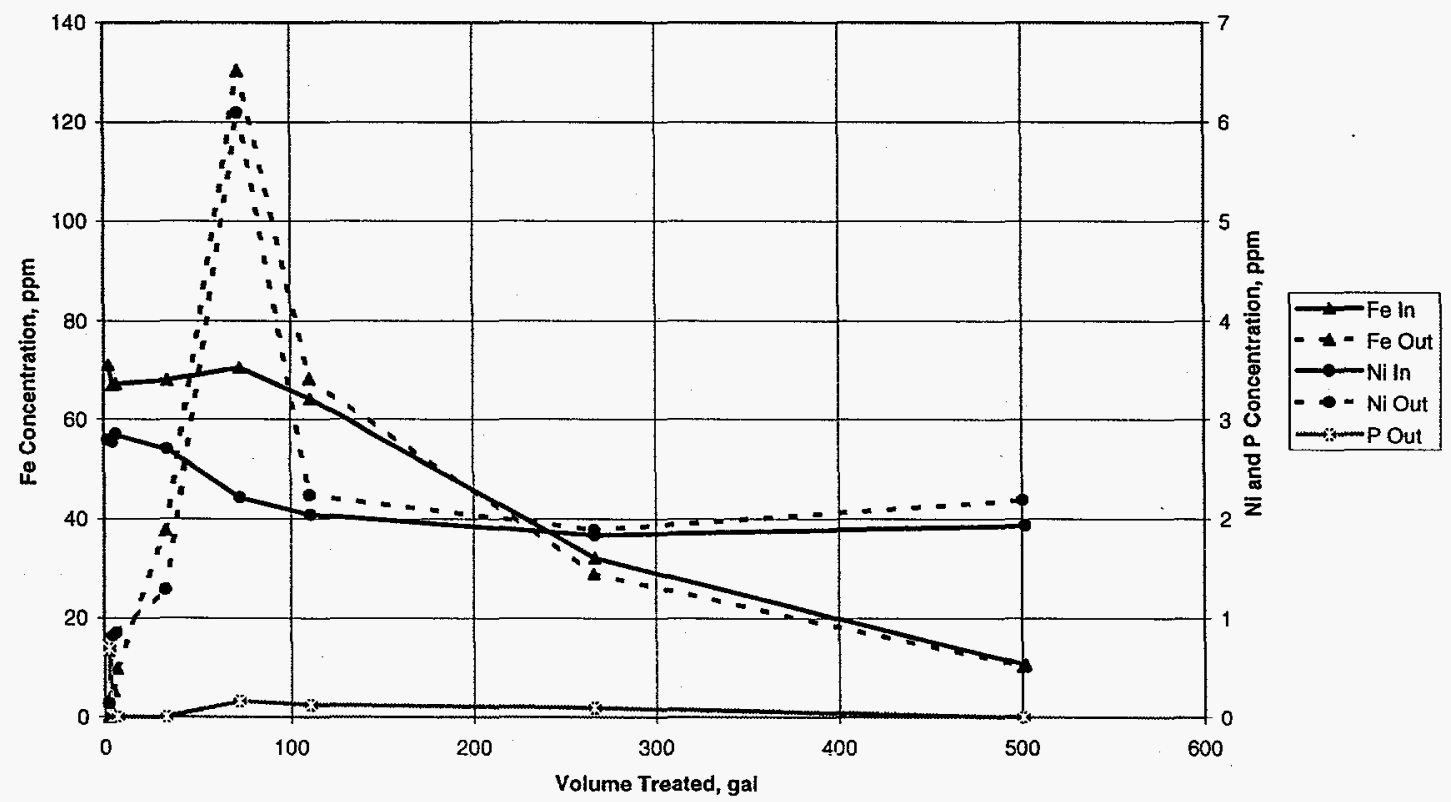


Figure 12. Ex-Situ Treatment Media Evaluation Run \#1 Contact Zeolite Media

(Train 2 Canister 2)

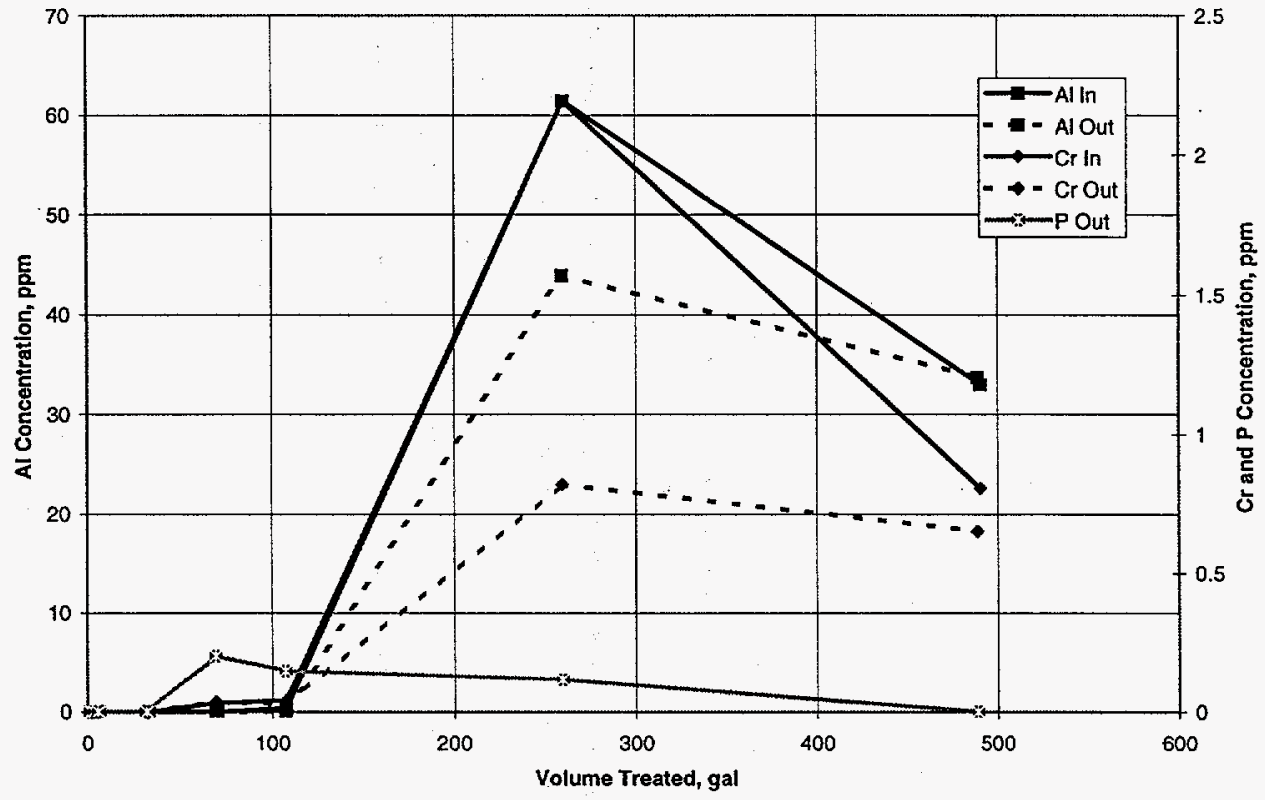

Figure 13. Ex-Situ Treatment Media Evaluation Run \#1 Contact Zeolite Media

(Train 2 Canister 2)

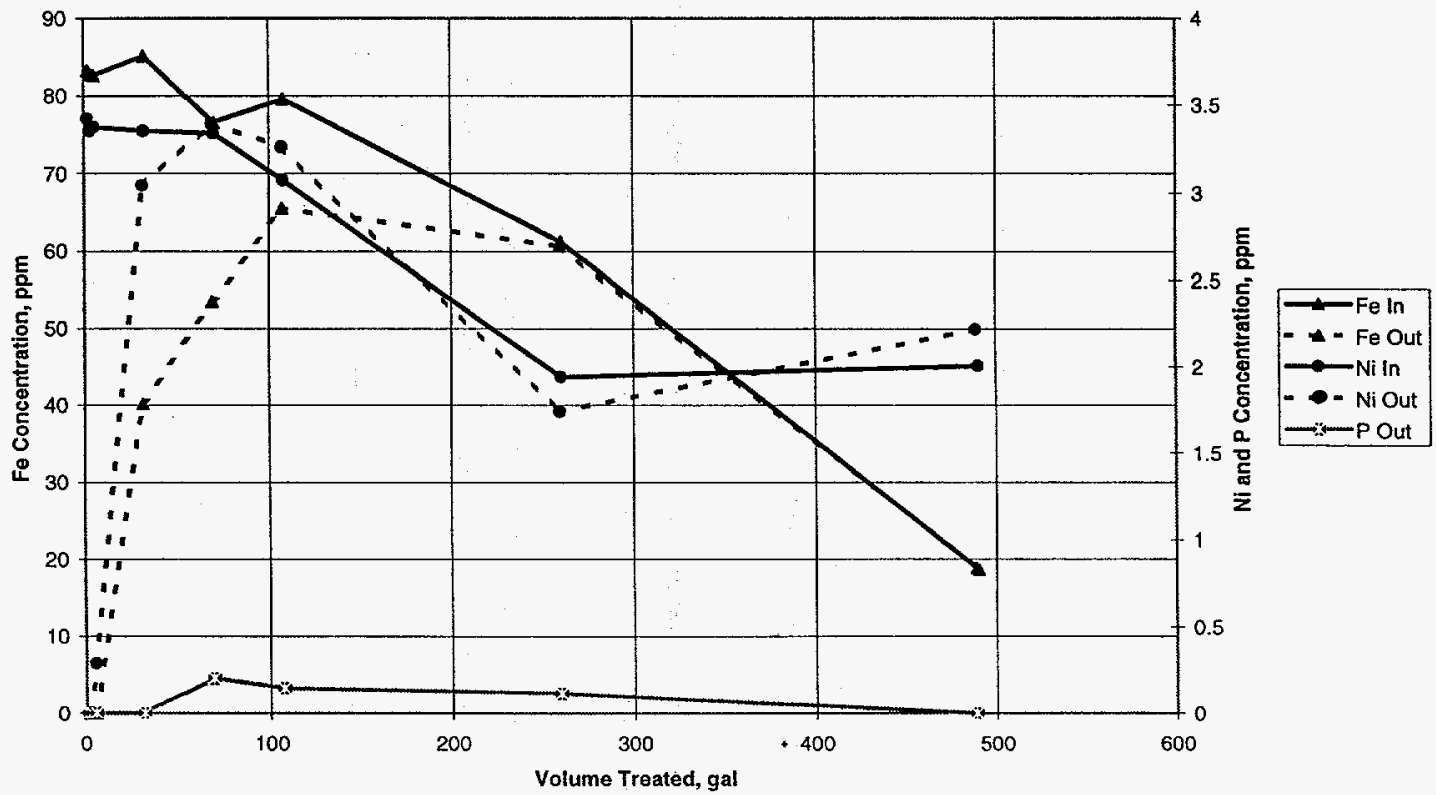


Figure 14. Ex-Situ Treatment Media Evaluation Run \#1 Contact Iron Media

(Train 3 Canister 2)

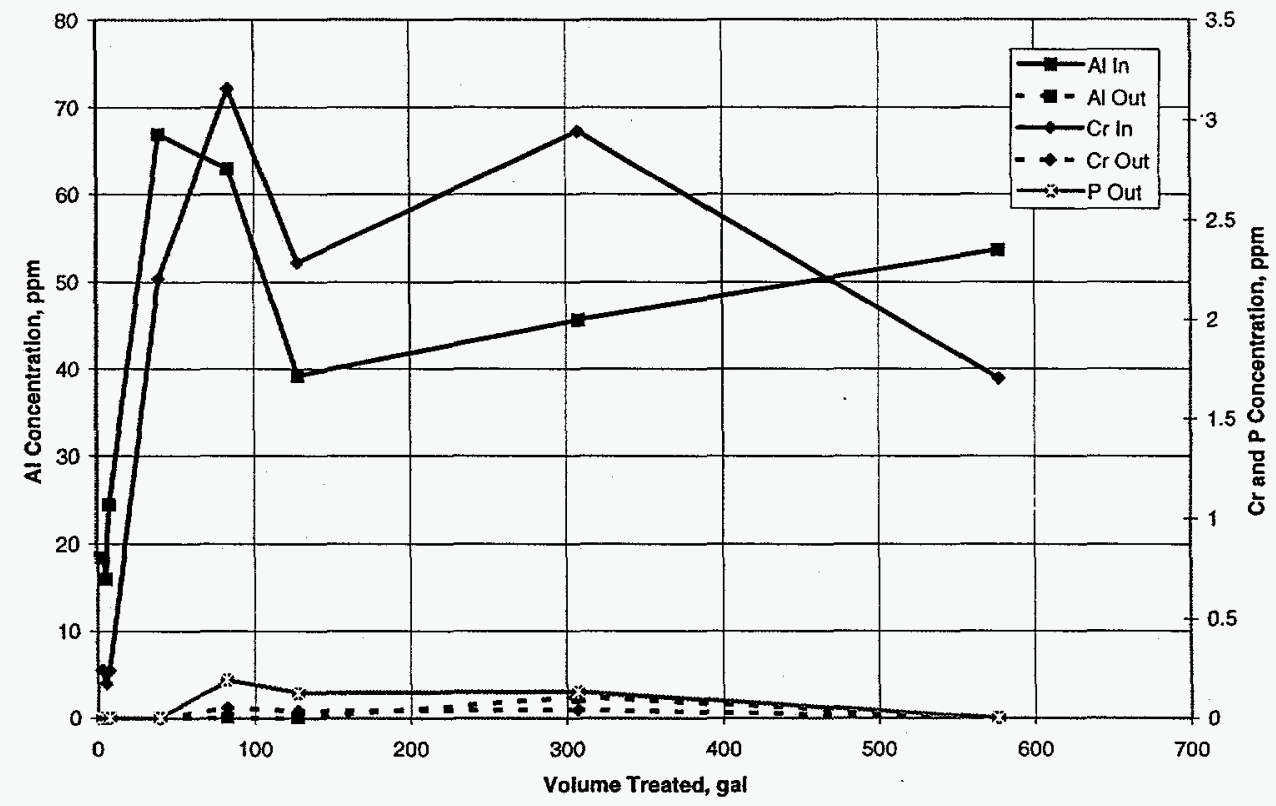

Figure 15. Ex-Situ Treatment Media Evaluation Run \#1

Contact Iron Media

(Train 3 Canister 2)

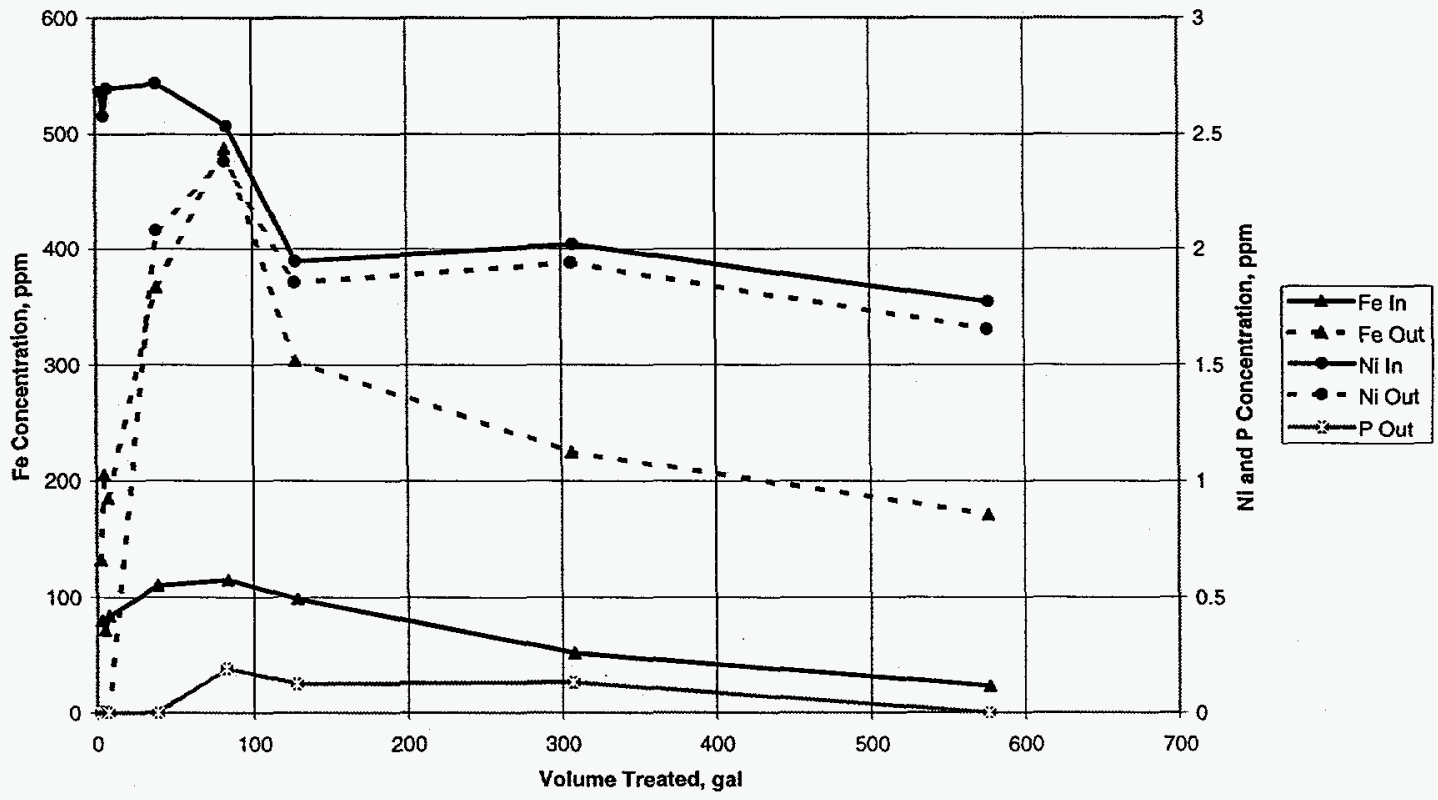


Figure 16. Ex-Situ Treatment Media Evaluation Run \#1

Contact Iron Media

(Train 4 Canister 2)

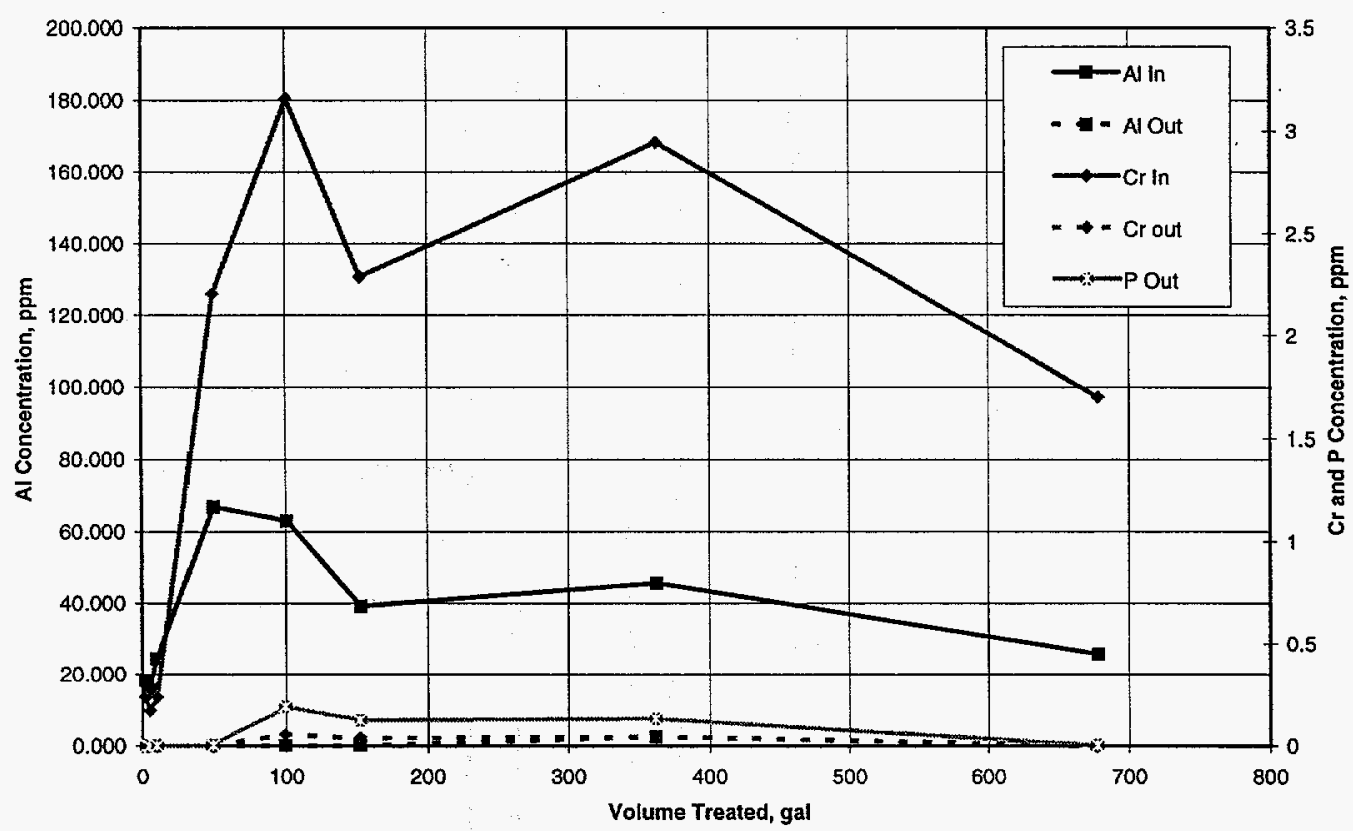

Figure 17. Ex-Situ Treatment Media Evaluation Run \#1

Contact Iron Media

(Train 4 Canister 2)

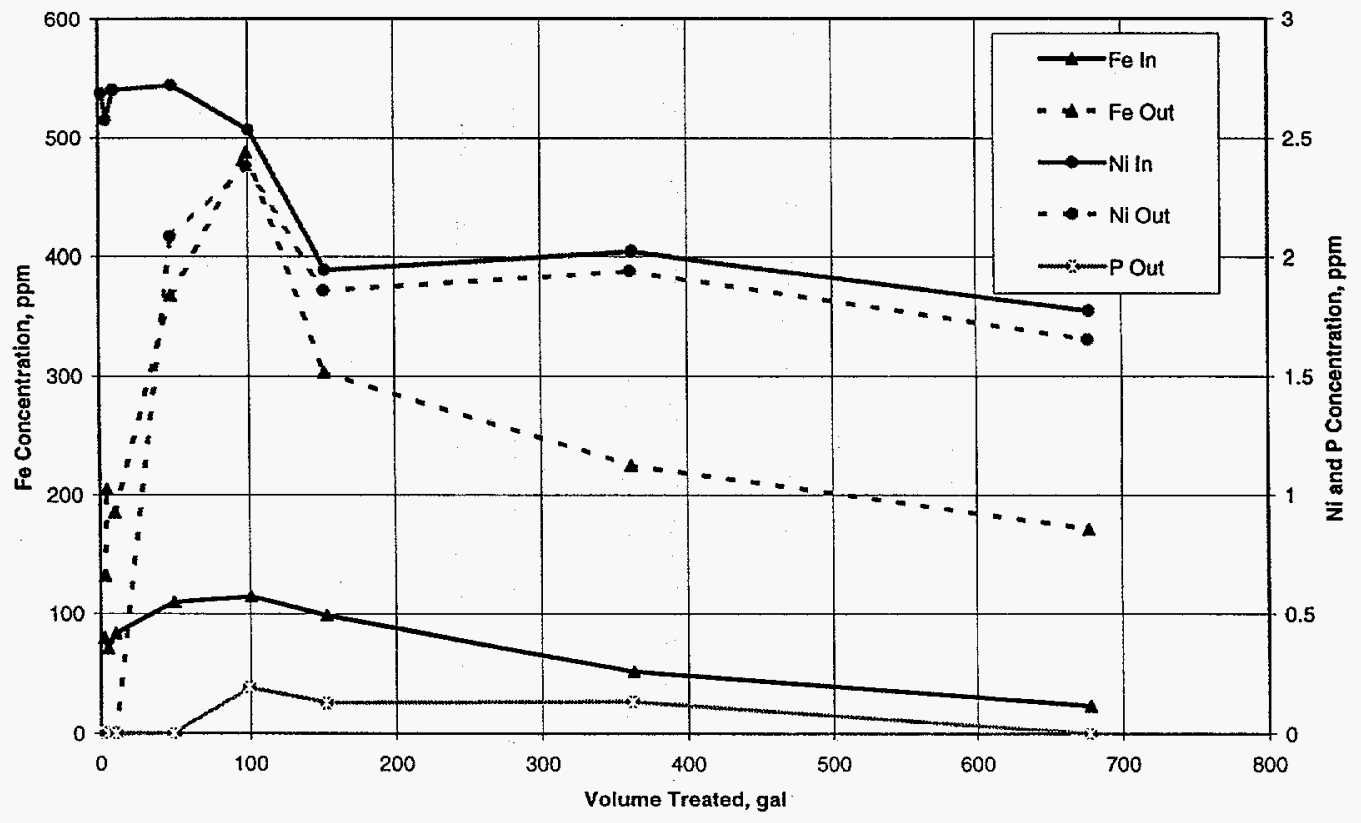




\section{Table 17}

\section{Preliminary Observations Field Run \#1 Contact Limestone (Trains 1,2,\&3 Canister 1)}

\begin{tabular}{|c|c|c|}
\hline Parameter & $\begin{array}{l}\text { Average } \\
\text { Change }\end{array}$ & Trend Comments \\
\hline $\mathrm{Al}$ & $74 \%$ removal & $\begin{array}{l}\text { \% removal highest the first two days at } 99 \% \text { and then variable between } 4 \\
\text { and } 99 \% \text { with an average of } 54 \% \text { with no discernable pattern; Conc. in } \\
\text { groundwater decreased over time }(\sim 180 \text { to } \sim 90 \mathrm{ppm}) \text {; Conc. in treated } \\
\text { water was below detection for the first two days and then variable between } \\
\sim 0.4 \text { and } \sim 110 \mathrm{ppm} \text { with an average of } 53 \mathrm{ppm} \text { with no discernable pattern. }\end{array}$ \\
\hline $\mathrm{Ca}$ & $\begin{array}{l}347 \% \\
\text { addition }\end{array}$ & $\begin{array}{l}\text { Decreasing } \% \text { addition over time except for third week; Conc. in } \\
\text { groundwater decreased over time ( } \sim 98 \text { to } 56 \mathrm{ppm}) \text {; Conc. in treated water } \\
\text { decreasing over time except for third week ( } \sim 600 \text { to } 150 \mathrm{ppm})\end{array}$ \\
\hline $\mathrm{Cr}$ & $79 \%$ removal & $\begin{array}{l}\% \text { removal highest the first two days at } 95 \% \text { then variable between } 5 \text { and } \\
98 \% \text { with an average of } 64 \% \text {; Conc. in groundwater fairly constant } \\
\text { between } 2 \text { and } 4 \mathrm{ppm} \text {; Conc. in treated water below detection for the first } \\
\text { two days and then variable between } \sim 0.04 \text { and } \sim 2.5 \mathrm{ppm} \text { with an average of } \\
1.2 \text { ppm with no discernable pattern. }\end{array}$ \\
\hline $\mathrm{Fe}$ & - & $\begin{array}{l}\text { Appears to be initially removed }(93 \%) \text { followed by addition; Conc. in } \\
\text { groundwater decreased over time }(\sim 170 \text { to } \sim 30 \mathrm{ppm}) \text {; Conc. in treated } \\
\text { water decreasing over time }(\sim 110 \text { to } \sim 20 \mathrm{ppm})\end{array}$ \\
\hline $\mathrm{Mg}$ & $25 \%$ addition & $\begin{array}{l}\text { Decreasing \% addition over time; Conc. in groundwater decreased over } \\
\text { time }(\sim 65 \text { to } \sim 30 \mathrm{ppm}) \text {; Conc. in treated water decreased over time }(\sim 95 \text { to } \\
\sim 30 \mathrm{ppm})\end{array}$ \\
\hline $\mathrm{Mn}$ & - & $\begin{array}{l}\text { Appears to be initially removed }(93 \%) \text { followed by addition; Conc. in } \\
\text { groundwater decreased over time }(\sim 14 \text { to } \sim 8 \mathrm{ppm}) \text {; Conc. in treated water } \\
\text { variable between } 7 \text { and } 13 \mathrm{ppm} \text { with an average of } 9.36 \mathrm{ppm}\end{array}$ \\
\hline $\mathrm{Ni}$ & - & $\begin{array}{l}\text { Appears to be initially removed }(90 \%) \text { followed by addition; Conc. in } \\
\text { groundwater decreased over time }(\sim 4 \text { to } \sim 1.5 \mathrm{ppm} \text { with an average of } 2.76) \text {; } \\
\text { Conc. in treated water variable between } \sim 0.1 \text { and } \sim 3.9 \text { ppm with an average } \\
\text { of } 2.23 \mathrm{ppm}\end{array}$ \\
\hline$P$ & None & None \\
\hline $\mathrm{Si}$ & $30 \%$ removal & $\begin{array}{l}\text { Decreasing \% removal over time except for third week; Conc. in } \\
\text { groundwater variable between } 40 \text { and } 60 \mathrm{ppm} \text { with an average of } 53 \mathrm{ppm} \text {; } \\
\text { Conc. in treated water variable between } \sim 6 \text { and } \sim 52 \mathrm{ppm} \text { with an average of } \\
37 \mathrm{ppm}\end{array}$ \\
\hline Sulfate & $10 \%$ removal & $\begin{array}{l}\text { Removal greatest at test start and then fairly constant; Conc. in } \\
\text { groundwater decreased over time }(\sim 2000 \text { to } 1000 \mathrm{ppm}) \text {; Conc. in treated } \\
\text { water decreased over time }(\sim 1900 \text { to } \sim 900 \mathrm{ppm})\end{array}$ \\
\hline $\mathrm{pH}$ & - & $\begin{array}{l}\text { Groundwater average }=3.8 \text { (fairly constant); treated water average }=5.7 \\
\text { (decreased over time from } \sim 7 \text { to }-4.5 \text { ) }\end{array}$ \\
\hline $\mathrm{DO}$ & - & $\begin{array}{l}\text { Groundwater average }=2.3 \text { (fairly constant); treated water average }=4.0 \\
\text { (decrease over time from } \sim 6 \text { to } \sim 2 \text {, which is essentially no change from } \\
\text { inlet) }\end{array}$ \\
\hline Eh & - & $\begin{array}{l}\text { Groundwater average }=488 \text { (fairly constant); treated water average }=286 \\
\text { (increase over time from } \sim 140 \text { to } \sim 410, \text { which is essentially no change from } \\
\text { inlet) }\end{array}$ \\
\hline
\end{tabular}


Table 18

\section{Preliminary Observations Field Run \#1 Contact Phosphate Rock Train 4 Canister 1}

\begin{tabular}{|c|c|c|}
\hline Parameter & $\begin{array}{l}\text { Average } \\
\text { Change } \\
\end{array}$ & Trend Comments \\
\hline $\mathrm{Al}$ & $78 \%$ removal & $\begin{array}{l}\% \text { removal highest the first day at } 98 \% \text { and then variable between } 58 \text { and } \\
74 \% \text { with an average of } 67 \% \text { with no discernable pattern; Conc. in } \\
\text { groundwater decreased over time }(\sim 180 \text { to } \sim 90 \mathrm{ppm}) \text {; Conc. in treated } \\
\text { water was below detection for the first day and then variable between } \sim 25 \\
\text { and } \sim 60 \mathrm{ppm} \text { with an average of } \sim 40 \mathrm{ppm} \text { with no discernable pattern. }\end{array}$ \\
\hline $\mathrm{Ca}$ & $\begin{array}{l}316 \% \\
\text { addition }\end{array}$ & $\begin{array}{l}\text { Decreasing } \% \text { addition over time; Conc. in groundwater decreased over } \\
\text { time }(-98 \text { to } 56 \mathrm{ppm}) \text {; Conc. in treated water decreasing over time ( } 400 \text { to } \\
200 \mathrm{ppm})\end{array}$ \\
\hline $\mathrm{Cr}$ & $59 \%$ removal & $\begin{array}{l}\% \text { removal highest the first day at } 96 \% \text { then variable between } 36 \text { and } 93 \% \\
\text { with an average of } \sim 40 \% \text {; Conc. in groundwater fairly constant between } 2 \\
\text { and } 4 \text { ppm; Conc. in treated water below detection for the first day and } \\
\text { then variable between } \sim 0.26 \text { and } \sim 2.7 \mathrm{ppm} \text { with an average of } \sim 2 \mathrm{ppm} \text { with } \\
\text { no discernable pattern. }\end{array}$ \\
\hline $\mathrm{Fe}$ & - & $\begin{array}{l}\text { Appears to be initially removed ( } 99 \%) \text { followed by addition; Conc. in } \\
\text { groundwater decreased over time }(\sim 170 \text { to } \sim 30 \mathrm{ppm}) \text {; Conc. in treated } \\
\text { water below detection for the first day and then variable between } 25 \text { and } \\
110 \mathrm{ppm} \text { with and average of } \sim 90\end{array}$ \\
\hline $\mathrm{Mg}$ & $\begin{array}{l}109 \% \\
\text { addition }\end{array}$ & $\begin{array}{l}\text { Decreasing \% addition over time; Conc. in groundwater decreased over } \\
\text { time }(\sim 65 \text { to } \sim 30 \mathrm{ppm}) ; \text { Conc. in treated water decreased over time }(\sim 265 \\
\text { to } \sim 30 \mathrm{ppm})\end{array}$ \\
\hline $\mathrm{Mn}$ & - & $\begin{array}{l}\text { Appears to be initially removed }(97 \%) \text { followed by addition; Conc. in } \\
\text { groundwater decreased over time }(\sim 14 \text { to } \sim 8 \mathrm{ppm}) \text {; Conc. in treated water } \\
<0.5 \mathrm{ppm} \text { the first day and then increases to } \sim 16 \text { followed by a decrease to } \\
\sim 8 \mathrm{ppm}\end{array}$ \\
\hline $\mathrm{Ni}$ & - & $\begin{array}{l}\text { Appears to be initially removed ( } 97 \%) \text { followed by essentially no change } \\
\text { from inlet or erratic removal/addition; Conc. in groundwater decreased over } \\
\text { time }(\sim 4 \text { to } \sim 1.5 \mathrm{ppm} \text { with an average of } 2.76 \mathrm{ppm}) \text {; Conc. in treated water } \\
\text { less than detection the first day and then variable concentration between } 1.7 \\
\text { to } 3.3 \mathrm{ppm} \text { with an average of } 2.3 \mathrm{ppm}\end{array}$ \\
\hline$P$ & $\begin{array}{l}4017 \% \\
\text { addition }\end{array}$ & $\begin{array}{l}\text { Decreasing } \% \text { addition over time; Conc. in groundwater }<0.67 \mathrm{ppm} \\
\text { throughout; Conc. in treated water initially } 60 \mathrm{ppm} \text { and may have stabilized } \\
\text { around } 5 \mathrm{ppm}\end{array}$ \\
\hline $\mathrm{Si}$ & $9 \%$ removal & $\begin{array}{l}\% \text { removal greatest at start of test; Conc. in groundwater variable between } \\
40 \text { and } 60 \mathrm{ppm} \text { with an average of } 53 \mathrm{ppm} \text {; Conc. in treated water variable } \\
\text { between } \sim 25 \text { and } \sim 60 \mathrm{ppm} \text { with an average of } 49 \mathrm{ppm}\end{array}$ \\
\hline Sulfate & - & $\begin{array}{l}\text { Appears to be initially removed followed by addition; Conc. in groundwater } \\
\text { decreased over time }(\sim 2000 \text { to } 1000 \mathrm{ppm}) \text {; Conc. in treated water } \\
\text { decreased over time }(\sim 1900 \text { to } \sim 1000 \mathrm{ppm})\end{array}$ \\
\hline $\mathrm{pH}$ & - & $\begin{array}{l}\text { Groundwater average }=3.8 \text { (fairly constant); treated water average }=5.2 \\
\text { (decreased over time from } \sim 6.5 \text { to } \sim 4.3 \text { ) }\end{array}$ \\
\hline DO & - & $\begin{array}{l}\text { Groundwater average }=2.3 \text { (fairly constant); treated water average }=4.9 \\
\text { (decreased over time from } \sim 9 \text { to } \sim 3 \text {; essentially no change from inlet) }\end{array}$ \\
\hline Eh & - & $\begin{array}{l}\text { Groundwater average }=488 \text { (fairly constant); treated water average }=366 \\
\text { (increased over time from } \sim 300 \text { to } \sim 440 ; \text { essentially no change from inlet) }\end{array}$ \\
\hline
\end{tabular}


Table 19

\section{Preliminary Observations Field Run \#1 Contact Peat Train 1 Canister 2}

\begin{tabular}{|c|c|c|}
\hline Parameter & $\begin{array}{l}\text { Average } \\
\text { Change }\end{array}$ & Trend Comments \\
\hline $\mathrm{Al}$ & $\begin{array}{l}35 \% \\
\text { removal }\end{array}$ & Removal decreased with time to addition \\
\hline $\mathrm{Ca}$ & None & None \\
\hline $\mathrm{Cr}$ & $\begin{array}{l}62 \% \\
\text { removal }\end{array}$ & $\begin{array}{l}\text { Removal was around } 80 \% \text { until the third week when it dropped } \\
\text { to } 10 \%\end{array}$ \\
\hline $\mathrm{Fe}$ & $\begin{array}{l}30 \% \\
\text { removal }\end{array}$ & $\begin{array}{l}\text { Removal greatest at test start; removal starts at } 99 \% \text { and } \\
\text { decreases with time; when the peat began contacting significant } \\
\mathrm{Al} \text {, it appears that the Fe was displaced by the } \mathrm{Al}\end{array}$ \\
\hline $\mathrm{Mg}$ & None & None \\
\hline $\mathrm{Mn}$ & $\begin{array}{l}11 \% \\
\text { addition }\end{array}$ & $\begin{array}{l}\text { Removal was around } 20 \% \text { for the first } 3 \text { days; when the peat } \\
\text { began contacting significant } \mathrm{Al} \text {, it appears that the } \mathrm{Mn} \text { was } \\
\text { displaced by the } \mathrm{Al}\end{array}$ \\
\hline$\overline{\mathrm{Ni}}$ & - & $\begin{array}{l}\text { Removal greatest at test start; removal starts at } 95 \% \text { and } \\
\text { decreased with time; when the peat began contacting } \\
\text { significant } \mathrm{Al} \text {, it appears that the } \mathrm{Ni} \text { was displaced by the } \mathrm{Al}\end{array}$ \\
\hline $\mathbf{P}$ & None & None \\
\hline $\mathrm{Si}$ & $\begin{array}{l}5 \% \\
\text { removal }\end{array}$ & Removal ranged between 1 and $15 \%$ with no obvious trends \\
\hline Sulfate & None & None \\
\hline $\mathrm{pH}$ & - & $\begin{array}{l}\text { Decrease from average of } 5.2 \text { to } 3.6 \text {; magnitude of decreases } \\
\text { decreased with time }\end{array}$ \\
\hline DO & - & $\begin{array}{l}\text { Appears to be initially increased followed by essentially no } \\
\text { change from inlet }\end{array}$ \\
\hline $\mathrm{Eh}$ & - & $\begin{array}{l}\text { Increased from average } 314 \text { to 558: magnitude of increase } \\
\text { decreased with time }\end{array}$ \\
\hline
\end{tabular}


Table 20

Preliminary Observations Field Run \#1 Contact Zeolite

Train 2 Canister 2

\begin{tabular}{|c|c|c|}
\hline Parameter & $\begin{array}{l}\text { Average } \\
\text { Change }\end{array}$ & Trend Comments \\
\hline $\mathrm{Al}$ & $\begin{array}{l}17 \% \\
\text { removal }\end{array}$ & Removal decreased with time to addition \\
\hline $\mathrm{Ca}$ & None & None \\
\hline $\mathrm{Cr}$ & $\begin{array}{l}26 \% \\
\text { removal }\end{array}$ & Removal ranged between 19 and $62 \%$ with no obvious trends \\
\hline $\mathrm{Fe}$ & $\begin{array}{l}50 \% \\
\text { removal }\end{array}$ & $\begin{array}{l}\text { Removal greatest at test start; removal started at } 99 \% \text { and } \\
\text { decreased with time to an addition }\end{array}$ \\
\hline $\mathrm{Mg}$ & None & None \\
\hline $\mathrm{Mn}$ & $\begin{array}{l}14 \% \\
\text { removal }\end{array}$ & $\begin{array}{l}\text { Removal was around } 88 \% \text { for the first day; when the peat } \\
\text { began contacting significant } \mathrm{Al} \text {, it appears that the } \mathrm{Mn} \text { was } \\
\text { displaced by the } \mathrm{Al}\end{array}$ \\
\hline $\mathrm{Ni}$ & $\begin{array}{l}36 \% \\
\text { removal }\end{array}$ & $\begin{array}{l}\text { Removal greatest at test start; removal started at } 96 \% \text { and } \\
\text { decreased with time to essentially nothing; it does not appear } \\
\text { that the nickel was displaced significantly by } \mathrm{Al}\end{array}$ \\
\hline$P$ & None & None \\
\hline $\mathrm{Si}$ & $\begin{array}{l}13 \% \\
\text { removal }\end{array}$ & Fairly constant throughout the test \\
\hline Sulfate & None & None \\
\hline $\mathrm{pH}$ & No change & No change (i.e. inlet equaled outlet) \\
\hline DO & - & DO increased through the zeolite from average of 3.14 to 6.08 \\
\hline $\mathrm{Eh}$ & - & $\begin{array}{l}\text { Initially had significant increase in Eh followed by lesser } \\
\text { increase over time }\end{array}$ \\
\hline
\end{tabular}


Table 21

Preliminary Observations Field Run \#1 Contact Iron Following Limestone Train 3 Canister 2

\begin{tabular}{|c|c|c|}
\hline Parameter & $\begin{array}{l}\text { Average } \\
\text { Change }\end{array}$ & Trend Comments \\
\hline $\mathrm{Al}$ & $\begin{array}{l}97 \% \\
\text { removal }\end{array}$ & $\begin{array}{l}\% \text { removal ranged between } 94 \text { and } 99 \% \text { with no obvious } \\
\text { trends; Conc. in inlet water was below detection for the first } \\
\text { day and then variable between } \sim 53 \text { and } \sim 108 \text { ppm with no } \\
\text { discernable pattern; Conc. in treated water below detection } \\
\text { throughout the test }\end{array}$ \\
\hline $\mathrm{Ca}$ & None & Essentially no \% removal or addition \\
\hline $\mathrm{Cr}$ & $\begin{array}{l}90 \% \\
\text { removal }\end{array}$ & $\begin{array}{l}\text { \% removal ranged between } 74 \text { and } 98 \% \text { with no obvious } \\
\text { trends; Conc. in inlet water was below detection for the first } \\
\text { day and then variable averaged } \sim 1.7 \mathrm{ppm} \text {; Conc. in treated } \\
\text { water either below detection or }<0.1 \text { throughout the test }\end{array}$ \\
\hline $\mathrm{Fe}$ & $\begin{array}{l}541 \% \\
\text { addition }\end{array}$ & Significant iron \% addition throughout \\
\hline $\mathrm{Mg}$ & None & Essentially no \% removal or addition \\
\hline $\mathrm{Mn}$ & $\begin{array}{l}11 \% \\
\text { addition }\end{array}$ & $\begin{array}{l}\% \text { addition ranged between } 4 \text { and } 38 \% \text { with no obvious trends; } \\
\text { Conc. in inlet water averaged } \sim 10.6 \text {; Conc. in treated water } \\
\text { averaged } \sim 11.7\end{array}$ \\
\hline $\mathrm{Ni}$ & $\begin{array}{l}63 \% \\
\text { removal }\end{array}$ & $\begin{array}{l}\text { \% removal decreases over time }(95 \text { to } 36 \%) \text {; Conc. in inlet } \\
\text { water decreases over time }(\sim 2.3 \text { to } \sim 1.5 \mathrm{ppm}) \text {; Conc. in treated } \\
\text { water initially below detection and then variable between } 0.6 \\
\text { and } 1.4 \mathrm{ppm}\end{array}$ \\
\hline $\mathrm{P}$ & None & Essentially no \% removal or addition \\
\hline $\mathrm{Si}$ & $\begin{array}{l}44 \% \\
\text { removal }\end{array}$ & $\begin{array}{l}\% \text { removal initially greatest and then fairly constant at } \sim 28 \% \text {; } \\
\text { Conc. in inlet water fairly constant at an average } \sim 43 \mathrm{ppm} ; \\
\text { Conc. in treated water lowest initially and then fairly constant } \\
\text { at an average } \sim 33 \mathrm{ppm}\end{array}$ \\
\hline Sulfate & $\begin{array}{l}8 \% \\
\text { removal }\end{array}$ & $\begin{array}{l}\% \text { removal initially greatest and then fairly constant at } \sim 5 \% \text {; } \\
\text { Conc. in inlet water fairly constant at an average } \sim 1375 \mathrm{ppm} \text {; } \\
\text { Conc. in treated water lowest initially and then fairly constant } \\
\text { at an average } \sim 1310 \mathrm{ppm}\end{array}$ \\
\hline $\mathrm{pH}$ & - & $\begin{array}{l}\text { Inlet average }=5.2 \text { (decreasing over time from } \sim 6.3 \text { to } \sim 4.4) ; \\
\text { treated water average }=7.1 \text { (fairly constant) }\end{array}$ \\
\hline $\mathrm{DO}$ & - & $\begin{array}{l}\text { Inlet average }=2.9 \text { (decreasing over time from } \sim 4 \text { to } \sim 2) ; \\
\text { treated water average }=1.3 \text { (increasing over time from }<1 \text { to } \\
\sim 3 \text { ) }\end{array}$ \\
\hline Eh & - & $\begin{array}{l}\text { Inlet average }=336 \text { (initially } \sim 200 \text { followed by } \sim 400) ; \text { treated } \\
\text { water average }=30 \text { (fairly constant) }\end{array}$ \\
\hline
\end{tabular}


Table 22

Preliminary Observations Field Run \#1 Contact Iron Following Phosphate Rock Train 4 Canister 2

\begin{tabular}{|c|c|c|}
\hline Parameter & $\begin{array}{l}\text { Average } \\
\text { Change }\end{array}$ & Trend Comments \\
\hline $\mathrm{Al}$ & $\begin{array}{l}94 \% \\
\text { removal }\end{array}$ & $\begin{array}{l}\% \text { removal ranged between } 86 \text { and } 99 \% \text { with no obvious } \\
\text { trends; Conc. in variable between } \sim 15 \text { and } \sim 66 \text { ppm with no } \\
\text { discernable pattern; Conc. in treated water below detection } \\
\text { except for third week }\end{array}$ \\
\hline $\mathrm{Ca}$ & None & Essentially no \% removal or addition \\
\hline $\mathrm{Cr}$ & $\begin{array}{l}97 \% \\
\text { removal }\end{array}$ & $\begin{array}{l}\% \text { removal ranged between } 92 \text { and } 98 \% \text { with no obvious } \\
\text { trends; Conc. in inlet water was below detection for the first } \\
\text { day and then variable averaged } \sim 2.4 \mathrm{ppm} \text {; Conc. in treated } \\
\text { water either below detection or }<0.1 \mathrm{ppm} \text { throughout the test }\end{array}$ \\
\hline $\mathrm{Fe}$ & $\begin{array}{l}264 \% \\
\text { addition }\end{array}$ & Significant iron \% addition throughout \\
\hline $\mathrm{Mg}$ & None & Essentially no \% removal or addition \\
\hline $\mathrm{Mn}$ & $\begin{array}{l}11 \% \\
\text { addition }\end{array}$ & $\begin{array}{l}\% \text { addition ranged between none and } 27 \% \text { with no obvious } \\
\text { trends; Conc. in inlet water averaged } \sim 11.2 ; \text { Conc. in treated } \\
\text { water averaged } \sim 12.4\end{array}$ \\
\hline $\mathrm{Ni}$ & $\begin{array}{l}41 \% \\
\text { removal }\end{array}$ & $\begin{array}{l}\% \text { removal decreases over time }(95 \text { to } \sim 5 \%) \text {; Conc. in inlet } \\
\text { water decreases over time }(\sim 2.7 \text { to } \sim 1.8 \mathrm{ppm}) \text {; Conc. in treated } \\
\text { water initially below detection and then variable between } 1.6 \\
\text { and } 2.4 \mathrm{ppm}\end{array}$ \\
\hline $\mathrm{P}$ & $\begin{array}{l}87 \% \\
\text { removal }\end{array}$ & $\begin{array}{l}\text { \% removal fairly constant; Conc. in inlet water variable with an } \\
\text { average of } \sim 2.6 \mathrm{ppm} \text {; Conc. in treated water either below } \\
\text { detection or }<0.2 \mathrm{ppm} \text { throughout the test }\end{array}$ \\
\hline $\mathrm{Si}$ & $\begin{array}{l}48 \% \\
\text { removal }\end{array}$ & $\begin{array}{l}\% \text { removal decreasing over time }(84 \text { to } \sim 20 \%) \text {; Conc. in inlet } \\
\text { water fairly constant at an average } \sim 53 \mathrm{ppm} \text {; Conc. in treated } \\
\text { water lowest initially and then fairly constant at an average } \sim 35 \\
\text { ppm }\end{array}$ \\
\hline Sulfate & $\begin{array}{l}7 \% \\
\text { removal }\end{array}$ & $\begin{array}{l}\% \text { removal fairly constant at } \sim 7 \% \text {; Conc. in inlet decreasing } \\
\text { over time }(\sim 1575 \text { to } \sim 1100 \mathrm{ppm}) ; \text { Conc. in treated water inlet } \\
\text { decreasing over time }(\sim 1540 \text { to } \sim 1010 \mathrm{ppm})\end{array}$ \\
\hline $\mathrm{pH}$ & - & $\begin{array}{l}\text { Inlet average }=4.9(\text { decreasing over time from } \sim 5.8 \text { to } \sim 4.0) \\
\text { treated water average }=7.0 \text { (fairly constant) }\end{array}$ \\
\hline DO & - & $\begin{array}{l}\text { Inlet average }=3.3(\text { decreasing over time from } \sim 5 \text { to } \sim 2) \\
\text { treated water average }=1.9 \text { (variable) }\end{array}$ \\
\hline Eh & - & $\begin{array}{l}\text { Inlet average }=366 \text { (initially } \sim 250 \text { followed by } \sim 450) ; \text { treated } \\
\text { water average }=35 \text { (fairly constant) }\end{array}$ \\
\hline
\end{tabular}




\subsection{Field Run \#2}

\subsubsection{Revised Design Concept following Field Run 1}

Based on the preliminary observations from Field Run \#1, it was determined that initial treatment by phosphate or limestone effectively removed metals from the groundwater. However, direct contact with the media is only effective for a short time because of armoring and cementing of the media. Figure 16 shows the revised non-contact concept that was to be implemented for the project. This conceptual design configuration consisted of a treatment basin and a precipitation trench. The treatment basin consisted of a permanently installed permeable treatment media. Direct rainfall and storm water runoff would act as the water source for the basin. The treatment basin was designed so that the contained permeable treatment media determined in the ex situ treatability study would provide a treatment media solution. The water would pass over the permeable treatment media in the basin and flow via gravity to the precipitation trench. The precipitation trench consisted of a perforated pipe in a gravel bed for the distribution of the treatment media solution to the contaminated groundwater. This trench provided an area of lower hydraulic head to push the treatment media solution into the contamination zone for groundwater treatment.

Figure 18

Upgradient Rechargeable NonContact GeoFix Cell

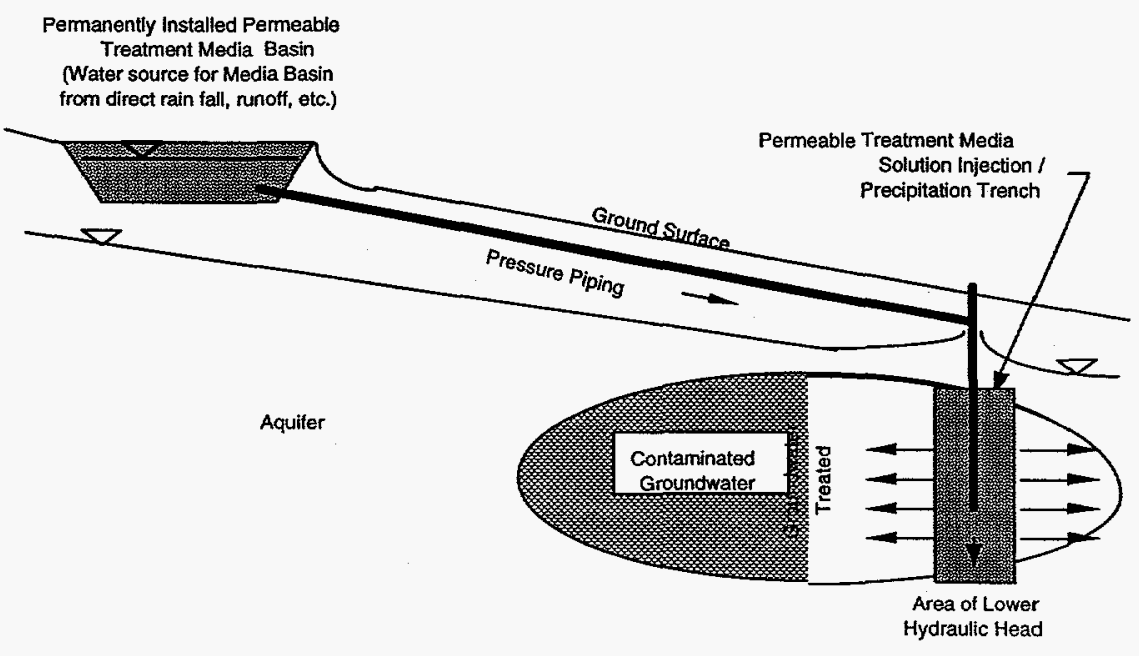




\subsubsection{Field Observations Field Run 2}

\subsubsection{Pressures/Plugging}

Pressures at the inlet and outlet to the canisters in Trains 1 and 2 remained fairly constant for the duration of the field run. The pressures for Run 2 ranged from -1.5 psig (at the down gradient discharge point) to $4.7 \mathrm{psig}$. The filter fabric was changed at the outlet to canister 2 for both trains on three occasions. No significant plugging or pressure problems were observed during the field run.

\subsubsection{Media}

The following field observations were noted after the run once the canisters were examined:

Train 1 Canister 1-Limestone

Inlet-The filter fabric shows no obvious signs of change except for a brown stain on the inlet/upstream side.

Outlet- A very small amount of white precipitate was coating the limestone. The water from the canister was a milky white. The media showed no obvious signs of change and the material was still loose.

Train 1 Canister 2 Packing Rings Inlet-The upstream side of the filter was lightly coated with a light green precipitate. The downstream side of the filter fabric showed no obvious signs of change.

Outlet-The packing rings were moderately coated with a green precipitate.

Train 2 Canister 1 Phosphate Rock

Inlet-The filter fabric shows no obvious signs of change except for a reddish stain on the inlet/upstream side of the canister.

Outlet-The phosphate rock material showed no obvious signs of change from the original material and was loose.

Train 2 Canister 2 Packing Rings

Inlet-The packing rings were moderately coated with a white and brown precipitate.

Outlet- The upstream side of the filter was coated with a $1 / 8$ " of an off white precipitate. The downstream side of the filter fabric showed no obvious signs of change. 


\subsubsection{Data Observations Field Run 2}

\subsubsection{Flow Summary}

The following table shows the average flows for each treatment train during this field run:

\begin{tabular}{|c|c|}
\hline Train & $\begin{array}{c}\text { Average Flows } \\
\mathrm{ml} / \mathrm{min}\end{array}$ \\
\hline 1 & 160 \\
\hline 2 & 143 \\
\hline
\end{tabular}

\subsubsection{Analytical Summary}

Figures 19-22 depict the primary contaminants (aluminum, chromium, iron, and nickel) through the canisters during this campaign. Preliminary observations are summarized below and shown in Tables 23-24.

\section{Limestone Train 1}

There was significant removal of aluminum and chromium throughout test. Iron and nickel were slightly removed throughout test and the removal may have increased at end of the test. Outlet calcium concentrations were initially greater than the inlet with an increase in the percent removal of over time. There was possibly a slight removal of manganese throughout the test. The $\mathrm{pH}$ increase was fairly consistent through out the test.

Phosphate Rock Train 2

There was significant removal of aluminum and chromium throughout test. There appears to possibly be an increase in removal toward the end of the test. Iron and nickel were slightly removed throughout test and the removal may have increased at end of the test. Outlet calcium concentrations were initially greater than the inlet with an increase in the percent removal of over time. The addition of phosphorus decreased over time. There was possibly a slight removal of manganese throughout the test. It appears that manganese may have had an increase in removal toward the end of the test. The $\mathrm{pH}$ increase was fairly consistent through out the test. 
Figure 19. Ex-Situ Treatment Media Evaluation Run \#2 Non Contact Limestone Media Train 1

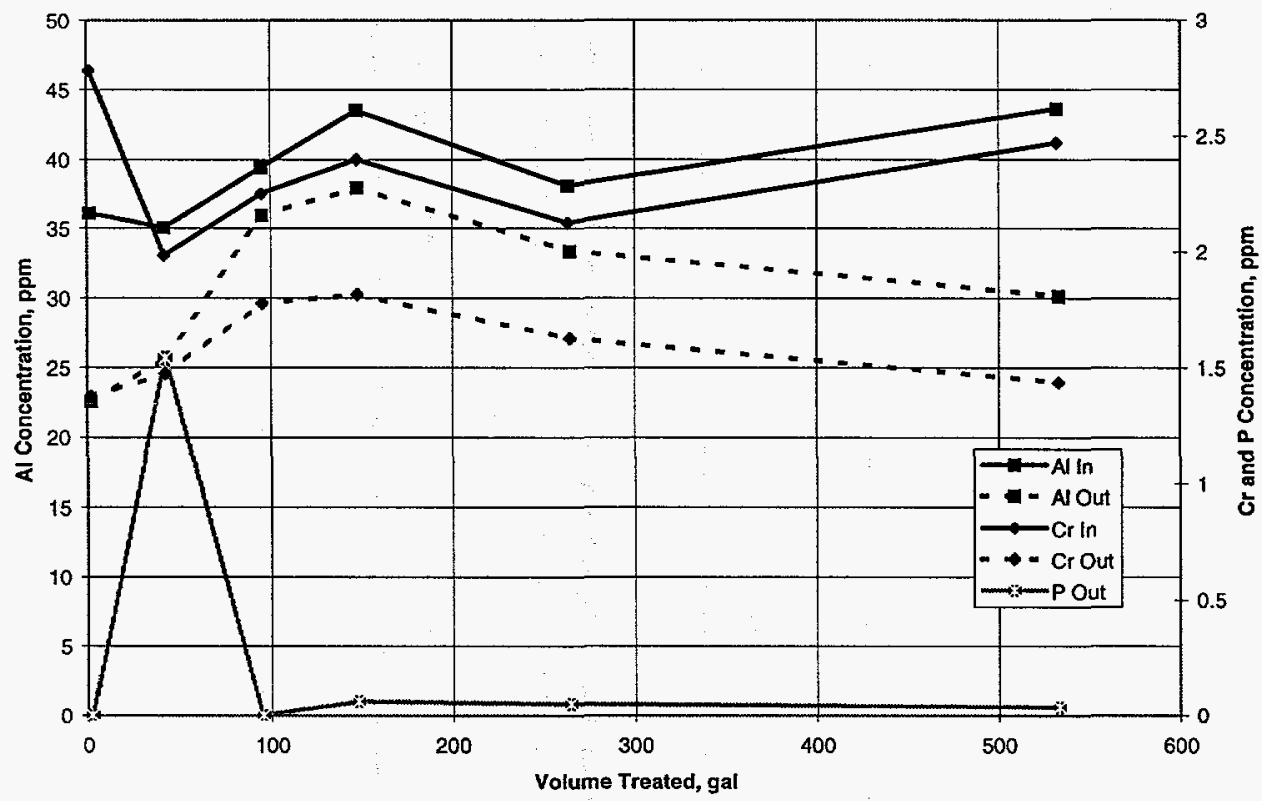

Figure 20. Ex-Situ Treatment Media Evaluation Run \#2 Non Contact Limestone Media Train 1

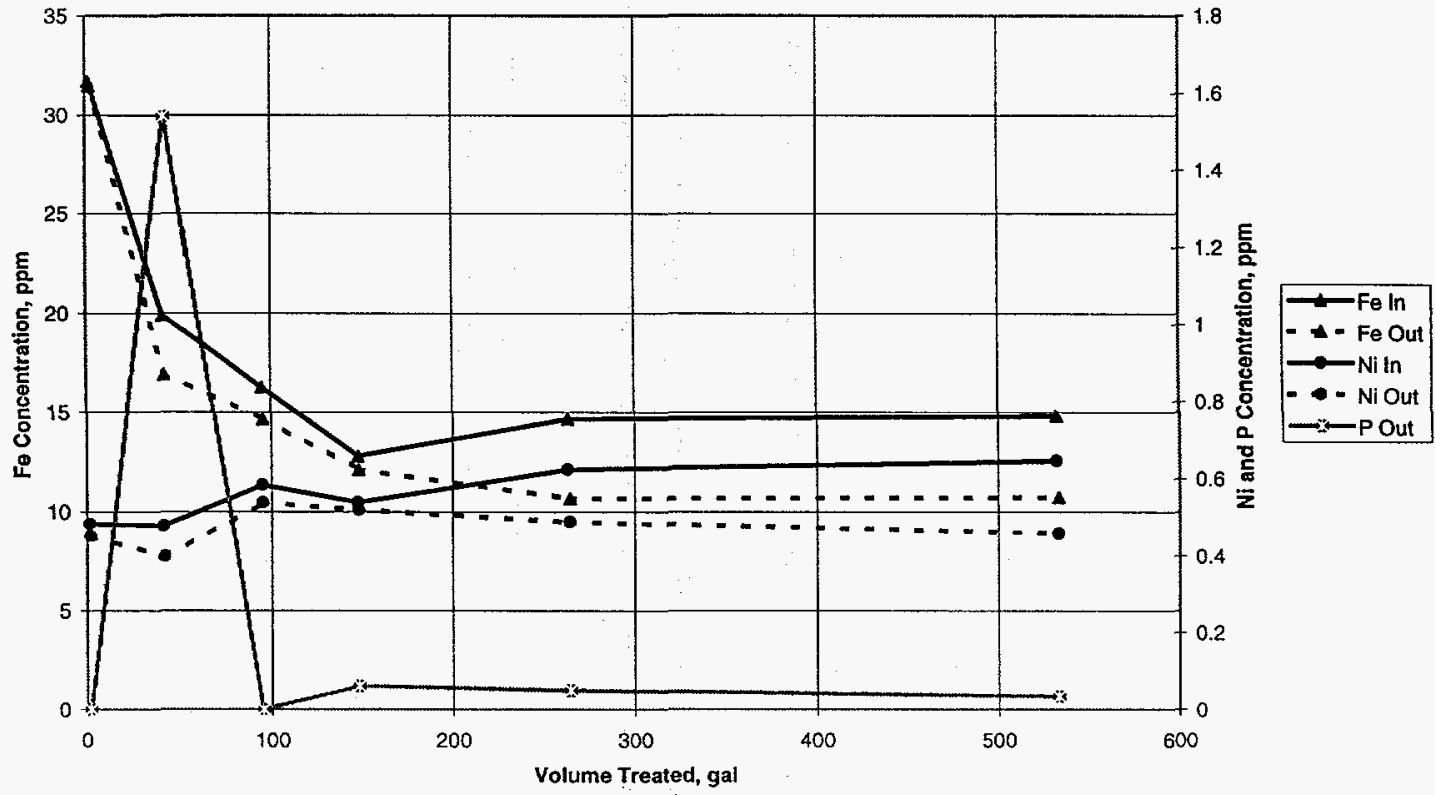


Figure 21. Ex-Situ Treatment Media Evaluation Run \#2 Non Contact Phosphate Rock Media Train 2

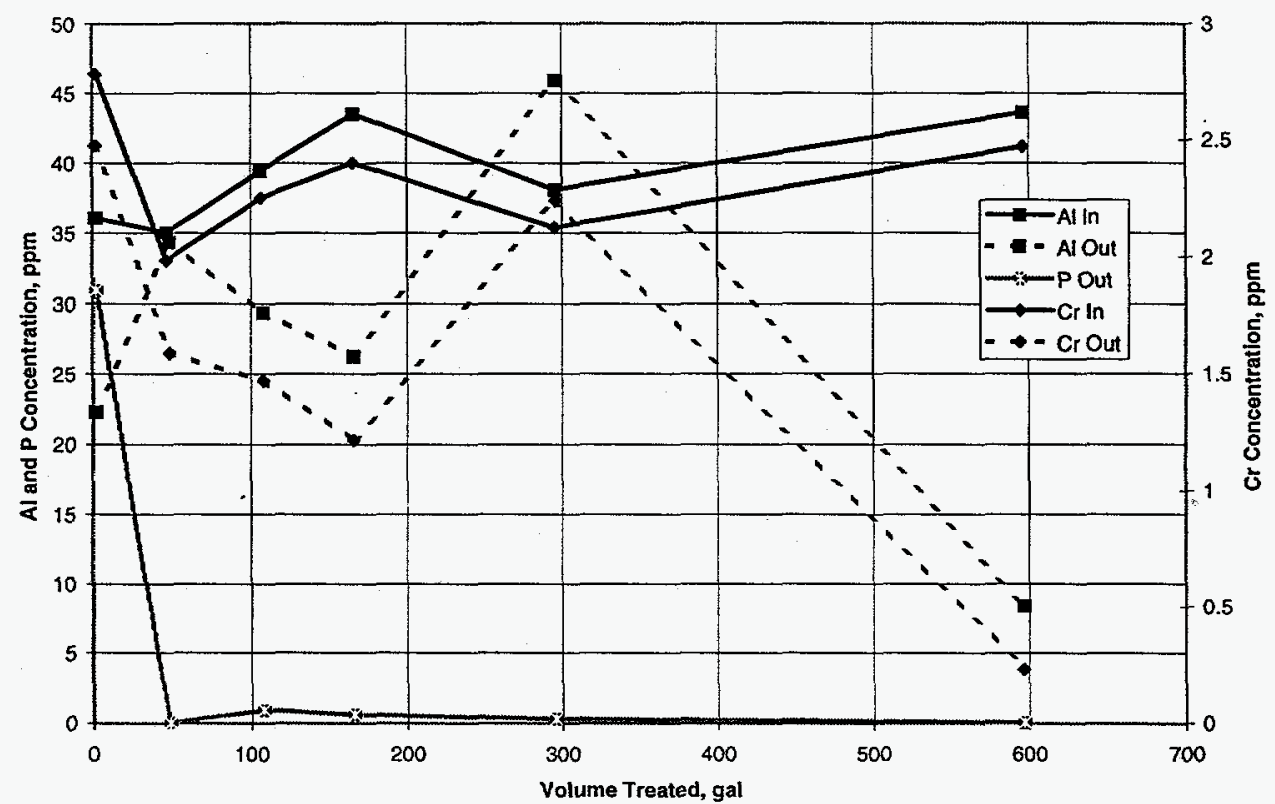

Figure 22. Ex-Situ Treatment Media Evaluation Run \#2 Non Contact Phosphate Rock Media Train 2

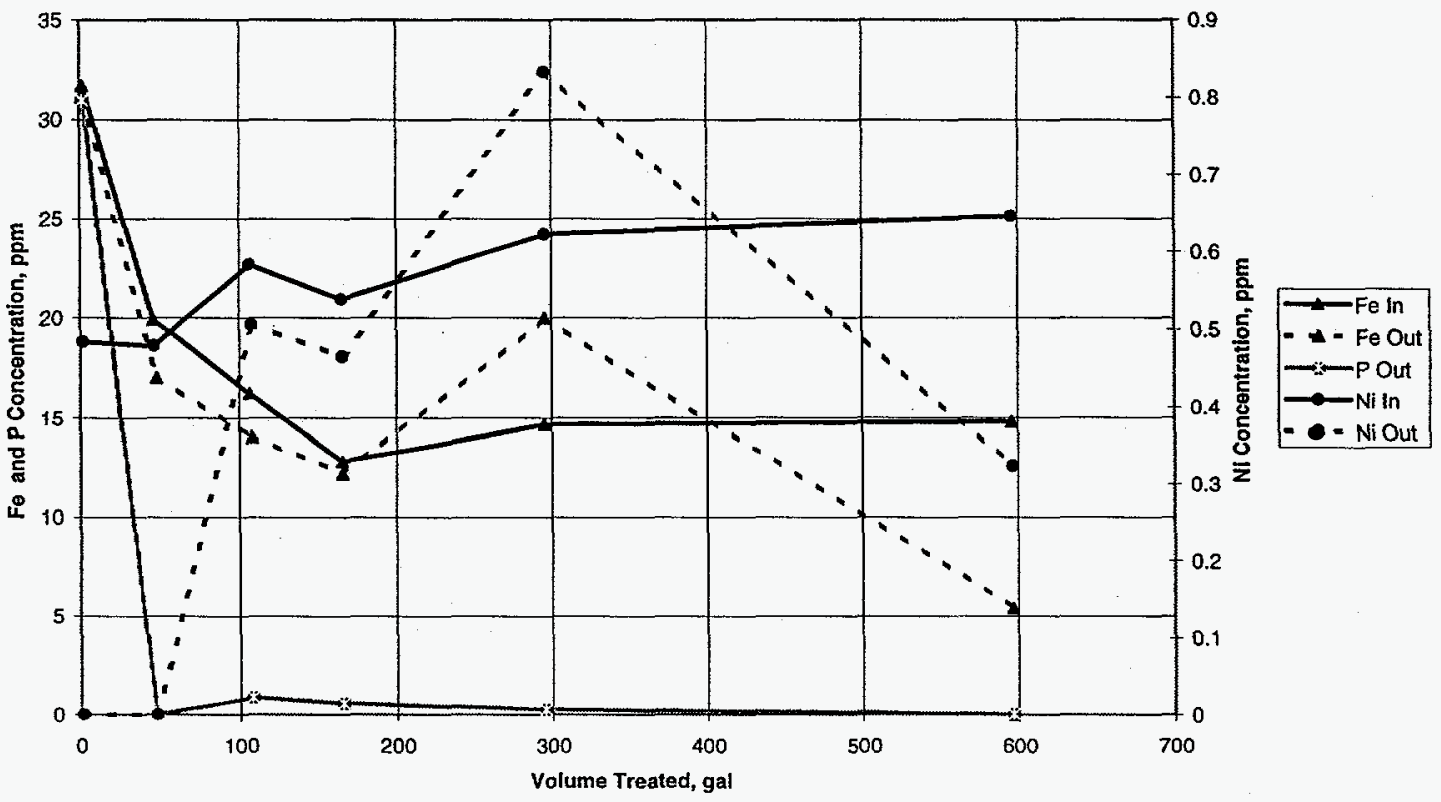


Table 23. Preliminary Observations Field Run \#2 Non-Contact Limestone Train 1

\begin{tabular}{|c|c|c|}
\hline Parameter & $\begin{array}{l}\text { Average } \\
\text { Change }\end{array}$ & Trend Comments \\
\hline $\mathrm{Al}$ & $\begin{array}{l}21 \% \\
\text { removal }\end{array}$ & $\begin{array}{l}\text { \% removal ranged from } \sim 9 \text { to } \sim 37 \% \text { with no obvious trends; Conc. } \\
\text { in groundwater fairly constant at an average of } 79 \mathrm{ppm} \text {; Conc. in } \\
\text { media solution less than detection throughout; Conc. in treated } \\
\text { water fairly constant at } 31 \mathrm{ppm}\end{array}$ \\
\hline $\mathrm{Ca}$ & $\begin{array}{l}14 \% \\
\text { addition }\end{array}$ & $\begin{array}{l}\text { Decreasing \% addition to increasing \% removal; Conc. in } \\
\text { groundwater slightly decreasing over time ( } 57 \text { to } 48 \text { ppm); Conc. in } \\
\text { media solution is highest the first day }(\sim 10) \text { and then is fairly } \\
\text { constant }(\sim 4) \text {; Conc. in treated water decreasing over time ( } 45 \text { to } 19 \\
\text { ppm) }\end{array}$ \\
\hline $\mathrm{Cr}$ & $\begin{array}{l}31 \% \\
\text { removal }\end{array}$ & $\begin{array}{l}\text { \% removal fairly constant; Conc. in groundwater fairly constant at } \\
\text { an average of } 4.67 \mathrm{ppm} \text {; Conc. in media solution less than detection } \\
\text { throughout; Conc. in treated water fairly constant at an average of } \\
1.58\end{array}$ \\
\hline $\mathrm{Fe}$ & $\begin{array}{l}14 \% \\
\text { removal }\end{array}$ & $\begin{array}{l}\text { Greatest \% removal toward end of test }(27 \%) \text {; Conc. in groundwater } \\
\text { is greatest the first two days and then fairly constant at } \sim 30 \mathrm{ppm} \text {; } \\
\text { Conc. in media solution less than detection throughout; Conc. in } \\
\text { treated water decreasing over time }(\sim 31 \text { to } \sim 11 \mathrm{ppm})\end{array}$ \\
\hline $\mathrm{Mg}$ & - & $\begin{array}{l}\text { Decreasing } \% \text { addition to increasing } \% \text { removal; Conc. in } \\
\text { groundwater greatest the first day and is then fairly constant at } \sim 25 \\
\text { ppm; Conc. in media solution decreasing over time }(4.5 \text { to } 0.5 \mathrm{ppm}) \text {; } \\
\text { Conc. in treated water decreasing over time }(\sim 17 \text { to } 10 \mathrm{ppm})\end{array}$ \\
\hline $\mathrm{Mn}$ & $\begin{array}{l}10 \% \\
\text { removal }\end{array}$ & $\begin{array}{l}\text { Greatest \% removal toward end of test }(27 \%) \text {; Conc. in groundwater } \\
\text { greatest the first day and is then fairly constant at } \sim 5.7 \mathrm{ppm} \text {; Conc. } \\
\text { in media solution less than detection for most of the test; Conc. in } \\
\text { treated water decreasing over time ( } 3.6 \text { to } 2.1 \mathrm{ppm})\end{array}$ \\
\hline $\mathrm{Ni}$ & $\begin{array}{l}14 \% \\
\text { removal }\end{array}$ & $\begin{array}{l}\text { Greatest \% removal toward end of test ( } 29 \%) \text {; Conc. in groundwater } \\
\text { slightly increasing over time ( } 1 \text { to } 1.3 \mathrm{ppm}) \text {; Conc. in media } \\
\text { solution less than detection throughout; Conc. in treated water fairly } \\
\text { constant at an average } 0.48\end{array}$ \\
\hline $\mathrm{P}$ & $\begin{array}{l}\sim 110 \% \\
\text { addition }\end{array}$ & $\begin{array}{l}\% \text { addition fairly constant; Conc. in groundwater averages } 0.06 \text {; } \\
\text { Conc. in media solution averages } 0.16 \text {; Conc. in treated water } \\
\text { averages } 0.06\end{array}$ \\
\hline $\mathrm{Si}$ & None & $\begin{array}{l}\text { No \% removal or addition; Conc. in groundwater averages } 38 \text {; } \\
\text { Conc. in media solution averages } 4 \text {; Conc. in treated water averages } \\
20\end{array}$ \\
\hline Sulfate & $\begin{array}{l}\text { Possible } \\
\text { addition }\end{array}$ & $\begin{array}{l}\text { Conc. in groundwater averages } 868 \text {; Conc. in media solution } \\
\text { averages } 5 ; \text { Conc. in treated water averages } 492\end{array}$ \\
\hline $\mathrm{pH}$ & - & $\begin{array}{l}\text { Groundwater ave }=3.8 ; \text { Media solution ave. }=7.81 ; \text { Treated water } \\
\text { ave }=4.26 ; \text { pHs fairly constant throughout the test }\end{array}$ \\
\hline $\mathrm{Eh}$ & - & $\begin{array}{l}\text { Groundwater ave. }=292 \text { (constant over time); Media solution ave. }= \\
300 \text { (increasing over time); treated water ave. }=283 \text { (constant over } \\
\text { time) }\end{array}$ \\
\hline
\end{tabular}




\section{Table 24. Preliminary Observations Field Run \#2 Non-Contact Phosphate Rock} Train 2

\begin{tabular}{|c|c|c|}
\hline Parameter & $\begin{array}{l}\text { Average } \\
\text { Change }\end{array}$ & Discharge Trend \\
\hline $\mathrm{Al}$ & $\begin{array}{l}27 \% \text { removal } \\
\text { predominately }\end{array}$ & $\begin{array}{l}\text { Greatest \% removal toward end of test }(81 \%) \text { and remainder of test fairly constant; } \\
\text { Conc. in groundwater fairly constant at an average of } 79 \mathrm{ppm} \text {; Conc. in media } \\
\text { solution less than detection for all but the first measurement; Conc. in treated water } \\
\text { fairly constant at average of } 28 \mathrm{ppm} \text { (end of test at } \sim 8 \mathrm{ppm} \text { ) }\end{array}$ \\
\hline $\mathrm{Ca}$ & $\begin{array}{l}126 \% \text { addition } \\
\text { predominately }\end{array}$ & $\begin{array}{l}\text { Initial } \% \text { addition ( } 711 \%) \text { much higher than other values and may have removal in } \\
\text { the last week of testing ( } 37 \%) \text {; Conc. in groundwater slightly decreasing over time } \\
(57 \text { to } 48 \mathrm{ppm}) \text {; Conc. in media solution decreases significantly over time ( } 375 \text { to } \\
3.5 \mathrm{ppm}) \text {; Conc. in treated water decreasing over time ( } 230 \text { to } 15 \mathrm{ppm})\end{array}$ \\
\hline $\mathrm{Cr}$ & $33 \%$ removal & $\begin{array}{l}\text { Potentially increased \% removal over time ( } 11 \text { to } 90 \%) \text {; Conc. in groundwater } \\
\text { fairly constant at an average of } 4.67 \mathrm{ppm} \text {; Conc. in media solution less than } \\
\text { detection for all but the first measurement; Conc. in treated water may be } \\
\text { decreasing over time ( } 2.5 \text { to } 0.23 \mathrm{ppm})\end{array}$ \\
\hline $\mathrm{Fe}$ & $10 \%$ removal & $\begin{array}{l}\text { Potentially increased \% removal over time ( } 2 \text { to } 63 \%) \text {; Conc. in groundwater is } \\
\text { greatest the first two days and then fairly constant at } \sim 30 \mathrm{ppm} \text {; Conc. in media } \\
\text { solution less than detection for all but the first measurement; Conc. in treated water } \\
\text { may be decreasing over time }(-31 \text { to } \sim 5 \mathrm{ppm})\end{array}$ \\
\hline $\mathrm{Mg}$ & - & $\begin{array}{l}\text { Decreasing } \% \text { addition to increasing } \% \text { removal ( } 597 \% \text { addition to } 45 \% \text { removal); } \\
\text { Conc. in groundwater greatest the first day and is then fairly constant at } \sim 25 \mathrm{ppm} \text {; } \\
\text { Conc. in media solution significantly decreasing over time }(\sim 180 \text { to } 0.6 \mathrm{ppm}) \text {; Conc. } \\
\text { in treated water decreasing over time }(-110 \text { to } \sim 7 \mathrm{ppm})\end{array}$ \\
\hline $\mathrm{Mn}$ & $\begin{array}{l}7 \% \text { removal } \\
\text { predominately }\end{array}$ & $\begin{array}{l}\% \text { removal appears to be increasing over time (none to } 48 \% \text { ); Conc. in groundwater } \\
\text { greatest the first day and is then fairly constant at }-5.7 \mathrm{ppm} \text {; Conc. in media } \\
\text { solution less than detection for half of the test; Conc. in treated water may be } \\
\text { decreasing over time ( } 3.8 \text { to } 1.5 \mathrm{ppm} \text { ) }\end{array}$ \\
\hline$\overline{\mathrm{Ni}}$ & $\begin{array}{l}14 \% \text { removal } \\
\text { predominately }\end{array}$ & $\begin{array}{l}\text { Greatest \% removal toward end of test }(50 \%) \text {; Conc. in groundwater slightly } \\
\text { increasing over time ( } 1 \text { to } 1.3 \mathrm{ppm}) ; \text { Conc. in media solution less than detection for } \\
\text { all but the first measurement; Conc. in treated water ranges from less than detection } \\
\text { for the first two days to a maximum of } \sim 0.8 \mathrm{ppm}\end{array}$ \\
\hline $\mathbf{P}$ & $\begin{array}{l}-16700 \% \\
\text { addition }\end{array}$ & $\begin{array}{l}\text { Decreasing } \% \text { addition over time ( } 92300 \text { to } 33 \%) \text {; Conc. in groundwater averages } \\
0.06 \text {; Conc. in media solution significantly decreasing over time }(\sim 72 \text { to } \sim 0.8 \mathrm{ppm}) \text {; } \\
\text { Conc. in treated water decreasing over time }(-31 \text { to } \sim 0.03 \mathrm{ppm})\end{array}$ \\
\hline $\mathrm{Si}$ & - & $\begin{array}{l}\text { May have significant Si from media initially, then essentially no \% removal or } \\
\text { addition; Conc. in groundwater averages } 38 \text {; Conc. in media solution decreasing } \\
\text { over time ( } \sim 56 \text { to } 3.5 \mathrm{ppm}) \text {; Conc. in treated water decreasing over time ( } \sim 48 \text { to } 12 \\
\mathrm{ppm} \text { ) }\end{array}$ \\
\hline Sulfate & - & $\begin{array}{l}\text { May have significant addition initially followed by potentially some removal }(\sim 160 \\
\text { addition to some potential removal); Conc. in groundwater averages } 868 \text {; Conc. in } \\
\text { media solution decreasing over time }(\sim 1400 \text { to } 11 \mathrm{ppm}) \text {; Conc. in treated water } \\
\text { initially } \sim 1100 \mathrm{ppm} \text { the first day followed by an average of } \sim 412 \mathrm{ppm} \text { for the rest of } \\
\text { the test }\end{array}$ \\
\hline $\mathrm{pH}$ & & $\begin{array}{l}\text { Groundwater ave. }=3.8 ; \text { Media solution ave }=6.70 ; \text { Treated water ave. }=4.25 \\
\text { pHs fairly constant throughout the test }\end{array}$ \\
\hline Eh & & $\begin{array}{l}\text { Groundwater ave. }=292 ; \text { Media solution ave. }=198 ; \text { Treated water ave. }=293 ; \\
\text { Eh's fairly constant throughout the test }\end{array}$ \\
\hline
\end{tabular}




\subsection{Field Run \#3}

\subsubsection{Conceptual Design}

Based on the preliminary observations from Field Run \#2 and Laboratory Tests \#1 \& \#2, it was determined that a non-contact treatment media, more soluble than rock phosphate or crushed limestone was needed for the waste stream and contaminants. Figure 21 shows the revised non-contact concept that was to be implemented for the project. This conceptual design configuration consisted of a combination treatment/precipitation trench. The treatment portion consisted of a removal permeable treatment media cell. Direct rainfall and storm water runoff would act as the water source for the trench. The treatment portion of the trench was designed so that the contained permeable treatment media, determined in the ex situ treatability study, would provide a treatment media solution. The water would pass over the permeable treatment media in the cell and flow via gravity to the precipitation portion of the trench. The precipitation portion of the trench consisted of an aggregate bed for the distribution of the treatment media solution and precipitation of the contaminants in the groundwater as it flows by the trench.

Figure 23

Upgradient Rechargeable NonContact GeoFix Cell

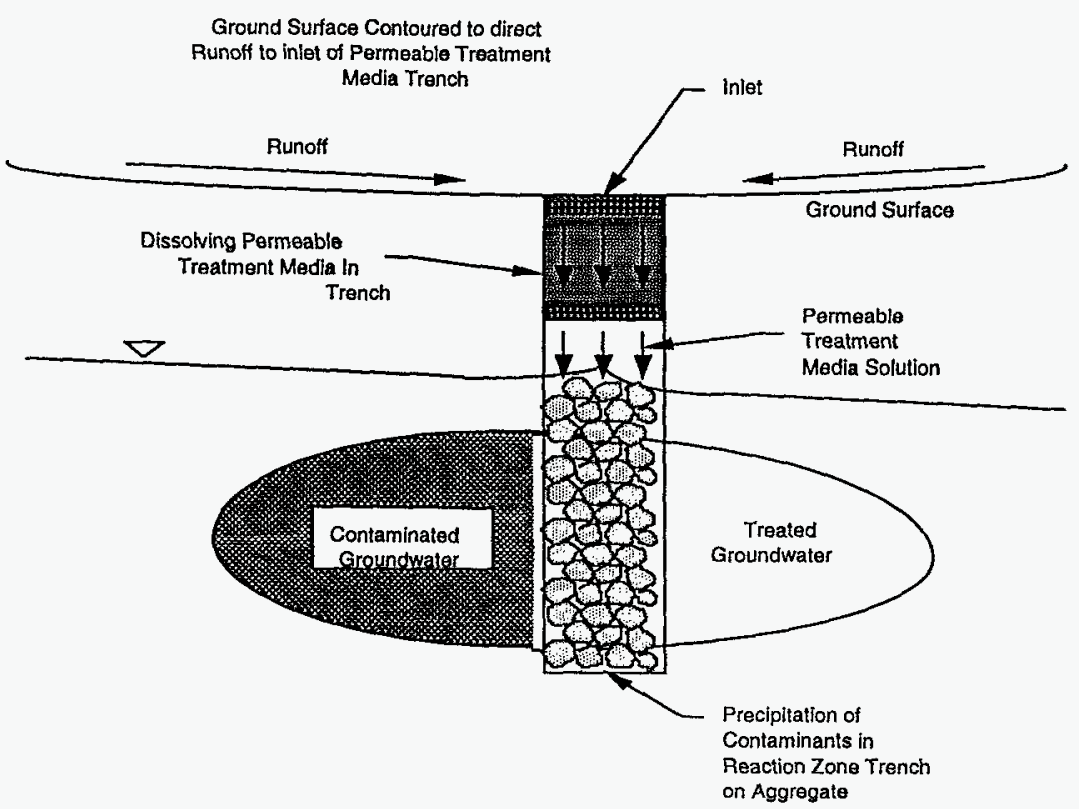




\subsubsection{Field Observations Field Run 3}

\subsubsection{Pressures/Plugging}

Pressures at the inlet and outlet to the canisters in Trains 1 and 2 remained constant for the duration of the field run. As previously stated, for all testing some negative pressures were observed due to the discharge line being down gradient from the test platform. The pressures for Run 3 ranged from -2.62 psig to -0.35 psig. A reading of .91 psig was observed when the discharge line in Train 2 became plugged. No other plugging or pressure problems were observed during field run 3 .

\subsubsection{Sludge Production}

For Run 3 it was anticipated that precipitates would flow through the system. Two layers of geonet with out the geotextile were used with the selected media at the bottom/inlet of each canister. This allowed for better flow-through. Five gallon catch tanks with screened bottoms and a layer of filter fabric were set up at the discharge points in the basin. Sludge was collected at an estimated minimum rate of 46 and $76 \mathrm{mg} / \mathrm{L}$ for the crushed limestone and crushed limestone/phosphate rock canisters, respectively. This value represents the minimum amount of sludge produced as catch tank overflows and filter bypasses did occur on occasion through out the test.

\subsubsection{Media}

The following field observations were noted after the run and the canisters examined:

Train 1 Canister 1 Limestone (>1/4"-3/4")

Inlet-The inlet (first 3-4" of limestone) had a yellowish with reddish brown patches indicating the zone below the solution inlet to the canister.

Outlet-The media past the first 4" was partially consolidated. The voids were filled with brownish-green precipitate, which occurred in two colors. Individual pebbles were coated in either brown or whitish green. 
Train 2 Canister 1 Lime stone (<1/4")/Phosphate Rock

Inlet-The first 2" was brownish precipitate and the media was partially consolidated.

Outlet- Past the first 2" there was a well-consolidated beige precipitate. The pore spaces were significantly filled. The precipitate occurred in two colors. Individual pebbles were coated in either a brown or whitish green precipitate at approximately a one to one ratio.

\subsubsection{Data Observations Field Run 3}

\subsubsection{Flow Summary}

The following table shows the average flows for each treatment train during this field run:

\begin{tabular}{|c|c|}
\hline Train & Average Flows $\mathrm{ml} / \mathrm{min}$ \\
\hline 1 & 204 \\
\hline 2 & 200 \\
\hline
\end{tabular}

\subsubsection{Analytical Summary}

Figures 24-27 depict the primary contaminants (aluminum, chromium, iron, and nickel) through the canisters during this campaign. Preliminary observations are summarized below and shown in Tables 25-26.

\section{Limestone}

There was significant aluminum and chromium removal throughout the test with some iron and nickel removal. There was a decrease in the calcium addition from the limestone over time. There was some manganese removal throughout the test. There was also significant phosphorus addition throughout the test since the phosphate is added in a soluble form. The $\mathrm{pH}$ increased constantly through the test.

\section{Limestone \& Phosphate Rock}

There was significant aluminum and chromium removal throughout the test with some iron removal. Outlet nickel concentrations are not consistent. Both removal and addition were noted throughout the test. There was a decrease in the calcium addition from the limestone and phosphate rock over time. There was some manganese removal throughout the test. There was also significant phosphorus addition throughout the test since the phosphate is added in a soluble form. Slight decreases in sulfate concentration were observed throughout the test. The $\mathrm{pH}$ increased constantly through the test. 
Figure 24. Ex-Situ Treatment Media Evaluation Run \#3 Limestone Media Train 1

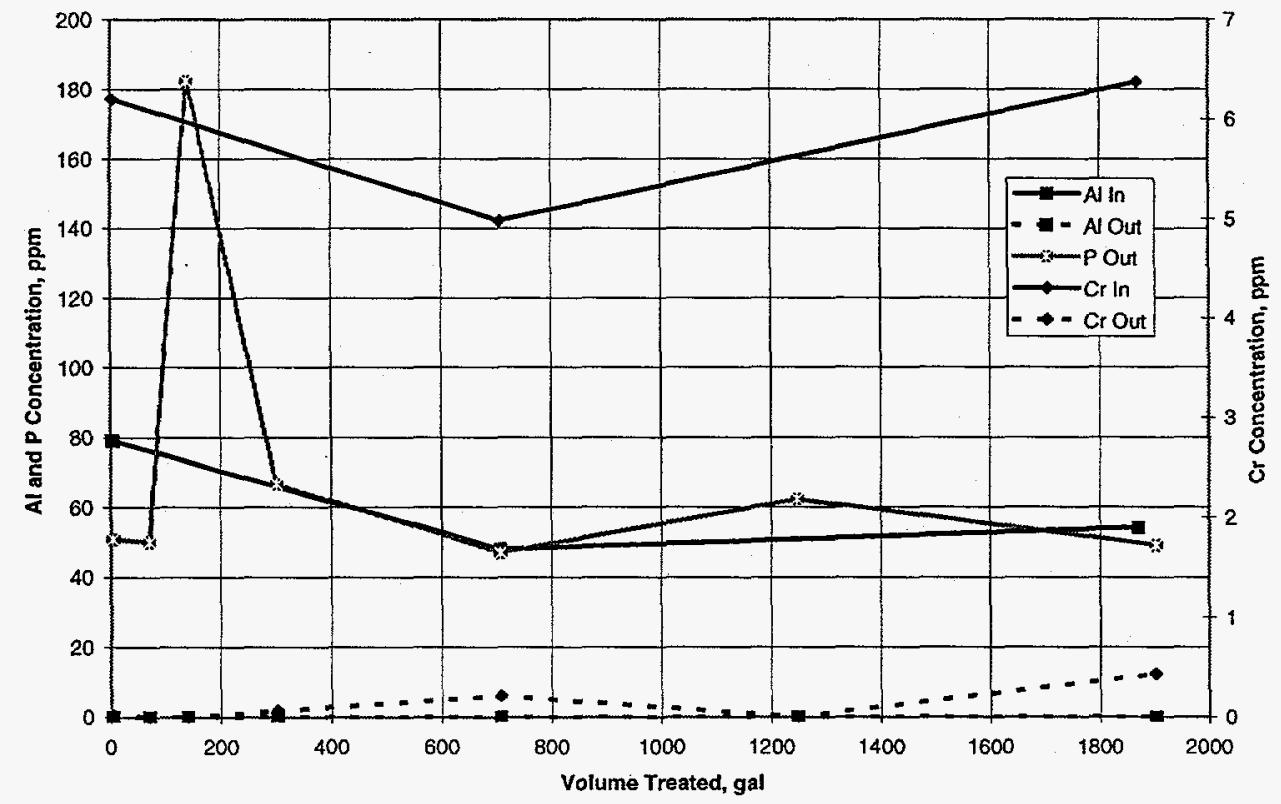

Figure 25. Ex-Situ Treatment Media Evaluation Run \#3 Limestone Media Train 1

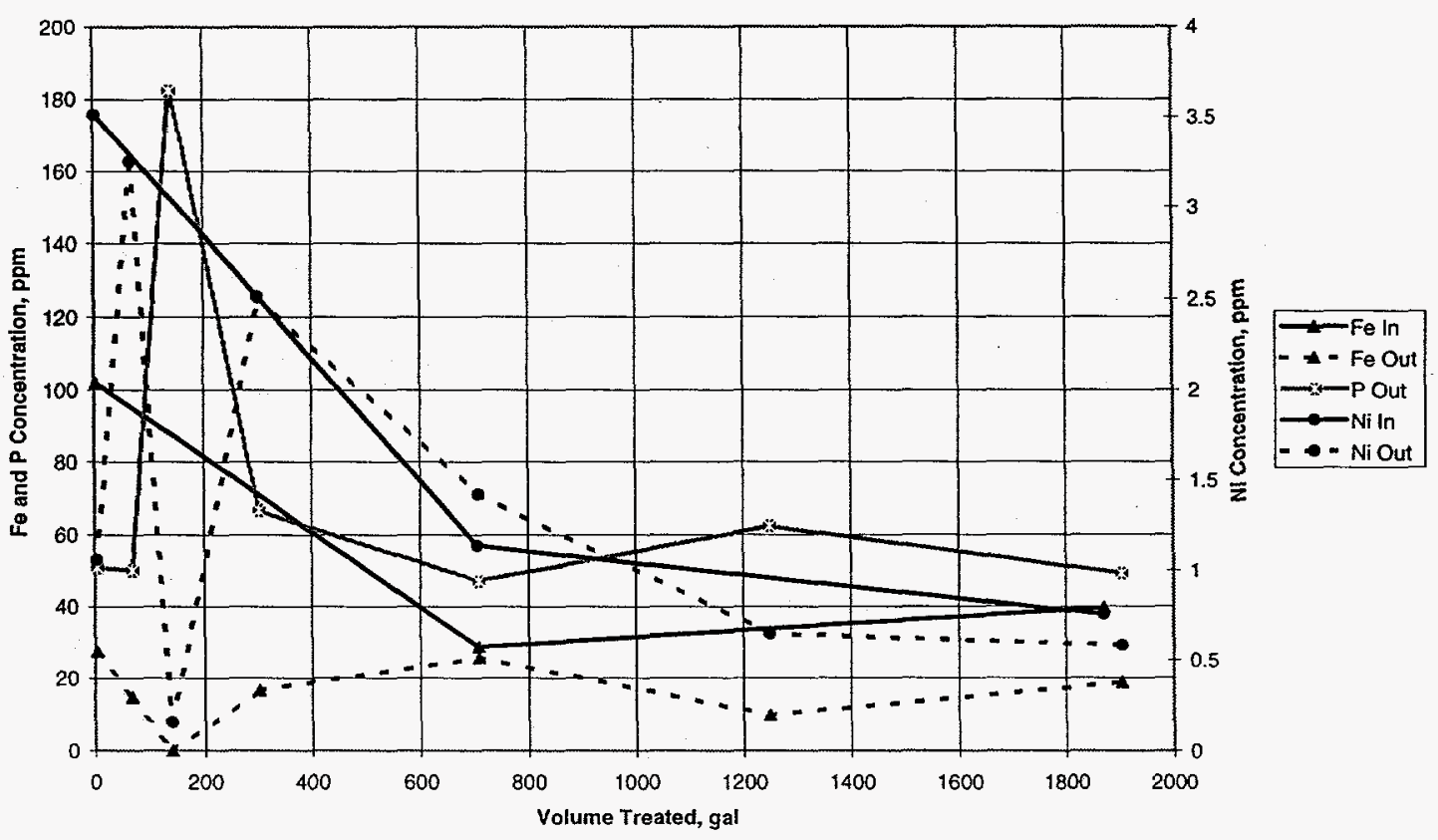


Figure 26. Ex-Situ Treatment Media Evaluation Run \#3

Limestone and Phosphate Rock Media Train 2

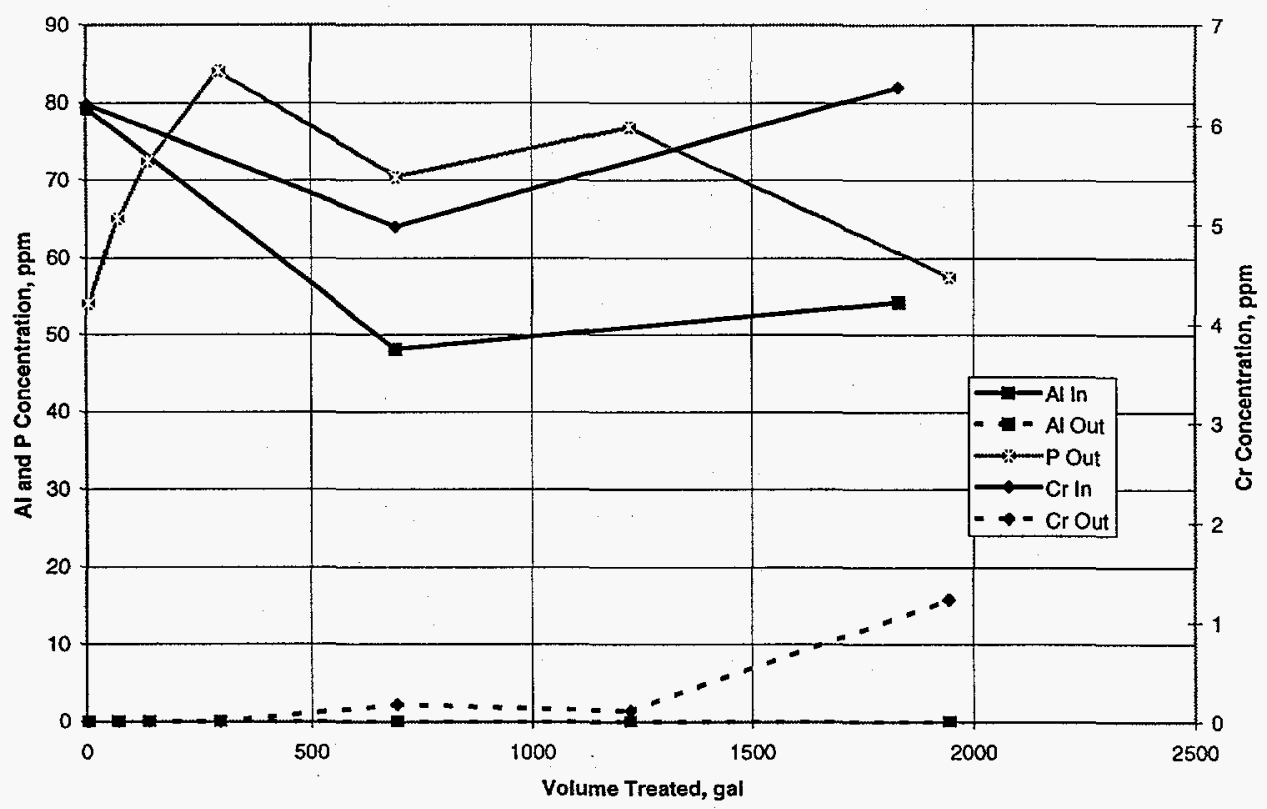

Figure 27. Ex-Situ Treatment Media Evaluation Run \#3 Limestone and Phosphate Rock Media Train 2
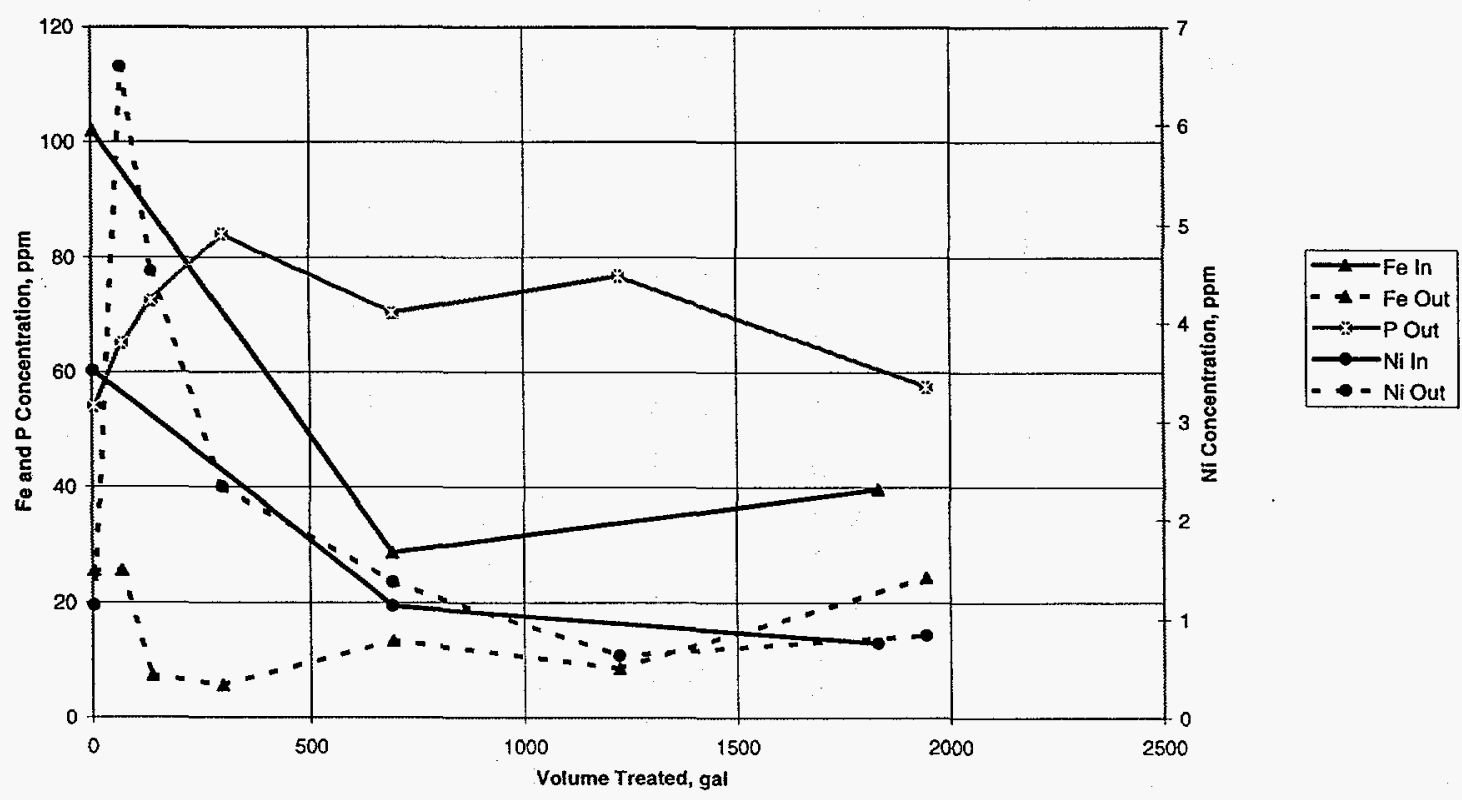


\section{Table 25. Preliminary Observations Field Run \#3 Train \#1 Limestone \& Potassium Phosphate Solution}

\begin{tabular}{|c|c|c|}
\hline Parameter & $\begin{array}{l}\text { Average } \\
\text { Change } \\
\end{array}$ & Trend Comments \\
\hline $\mathrm{Al}$ & $99 \%$ removal & $\begin{array}{l}\text { Constant \% removal ; Conc. in groundwater greatest the first } 2 \text { days }(\sim 150 \mathrm{ppm}) \\
\text { and fairly constant the rest of the test }(\sim 110 \mathrm{ppm}) \text {; Conc. in media solution below } \\
\text { detection }(<0.301 \mathrm{ppm}) \text {; Conc. in treated water below detection throughout the test } \\
(<0.301 \mathrm{ppm})\end{array}$ \\
\hline $\mathrm{Ca}$ & $87 \%$ addition & $\begin{array}{l}\% \text { addition highest first day ( } 209 \%) \text {; Conc. in groundwater greatest the first } 2 \text { days } \\
(\sim 104 \mathrm{ppm}) \text { and fairly constant the rest of the test }(\sim 67 \mathrm{ppm}) \text {; Conc. in media } \\
\text { solution fairly constant }(2.4 \mathrm{ppm}) \text {; Conc. in treated water greatest the first day }(\sim 210 \\
\text { ppm) and variable around } 50 \mathrm{ppm} \text { the rest of the test }\end{array}$ \\
\hline $\mathrm{Cr}$ & $98 \%$ removal & $\begin{array}{l}\text { \% removal was lowest the last week }(93 \%) \text {; Conc. in groundwater increased with } \\
\text { time }(8-14 \mathrm{ppm}) ; \text { Conc. in media solution below detection }(<0.060 \mathrm{ppm}) ; \text { Conc. in } \\
\text { treated water appears to increase over time }(0.020 \text { to } 0.431 \mathrm{ppm})\end{array}$ \\
\hline $\mathrm{Fe}$ & $64 \%$ removal & $\begin{array}{l}\text { Fairly constant \% removal; Conc. in groundwater greatest the first } 2 \text { days }(\sim 190 \\
\text { ppm) and fairly constant the rest of the test }(\sim 80 \mathrm{ppm}) \text {; Conc. in media solution } \\
\text { increased to above detection the last two weeks of the test }(<0.046-0.293) \text {; Conc. } \\
\text { in treated water fairly constant }(19 \mathrm{ppm})\end{array}$ \\
\hline $\mathrm{Mg}$ & - & $\begin{array}{l}\text { Appears to be initially added followed by a low net removal; Conc. in groundwater } \\
\text { greatest the first } 2 \text { days }(\sim 60 \mathrm{ppm}) \text { and fairly constant the rest of the test }(\sim 37 \mathrm{ppm}) \text {; } \\
\text { Conc. in media solution increased the last two weeks of the test }(0.2 \text { to } 2.8 \mathrm{ppm}) \text {; } \\
\text { Conc. in treated water greatest the first } 2 \text { days }(-30 \mathrm{ppm}) \text { and fairly constant the rest } \\
\text { of the test }(-18 \mathrm{ppm})\end{array}$ \\
\hline $\mathrm{Mn}$ & $34 \%$ removal & $\begin{array}{l}\text { Fairly constant \% removal; Conc. in groundwater greatest the first } 2 \text { days }(-10 \mathrm{ppm}) \\
\text { and fairly constant the rest of the test }(6-7 \mathrm{ppm}) ; \text { Conc. in media solution } \\
\text { increased to above detection the last two weeks of the test }(<0.028-0.093 \mathrm{ppm}) \text {; } \\
\text { Conc. in treated water fairly constant }(2.5 \mathrm{ppm})\end{array}$ \\
\hline $\mathrm{Ni}$ & $25 \%$ removal & $\begin{array}{l}\text { Fairly constant \% removal; Conc. in groundwater appeared to decrease with time ( } 8 \\
-2 \mathrm{ppm}) \text {; Conc. in media solution below detection }(<0.097 \mathrm{ppm}) \text {; Conc. in treated } \\
\text { water decreased with time }(3.2-0.6 \mathrm{ppm})\end{array}$ \\
\hline $\bar{P}$ & $\begin{array}{l}-27,500 \% \\
\text { addition }\end{array}$ & $\begin{array}{l}\text { \% addition highest the first day and last week }(-43,000 \%) \text { and fairly constant in } \\
\text { between }(-20,000 \%) ; \text { Conc. in groundwater fairly constant }(0.37 \mathrm{ppm}) \text {; Conc. in } \\
\text { media solution constant ( } 235 \mathrm{ppm}) \text {; Conc. in treated water fairly constant ( } 54 \mathrm{ppm})\end{array}$ \\
\hline$\overline{\mathrm{Si}}$ & $4 \%$ removal & $\begin{array}{l}\text { Variable \% removal and addition if any; Conc. in groundwater constant ( } 78 \mathrm{ppm}) \text {; } \\
\text { Conc. in media solution fairly constant (5.6 ppm); Conc. in treated water constant } \\
(38 \mathrm{ppm})\end{array}$ \\
\hline Sulfate & $\begin{array}{l}\text { Possible } \\
\text { addition }\end{array}$ & $\begin{array}{l}\text { Appears to be initially removed }(\sim 18 \%) \text { followed by addition }(\sim 23 \%) \text {; Conc. in } \\
\text { groundwater greatest the first } 2 \text { days }(-2400) \text { and fairly constant the rest of the test } \\
(-1275 \text { ppm); Data for Conc. in media solution suspect; Conc. in treated water } \\
\text { greatest the first } 2 \text { days }(-980 \mathrm{ppm}) \text { and fairly constant the rest of the test }(\sim 790 \\
\text { ppm) }\end{array}$ \\
\hline $\mathrm{pH}$ & - & $\begin{array}{l}\text { Media solution ave. }=7.92(8.45 \text { by omitting the lowest recorded } \mathrm{pH} \text {; fairly } \\
\text { constant); groundwater ave. }=3.23 \text { (initial two day's groundwater } \mathrm{pHs} \text { somewhat } \\
\text { low: } \sim 2.75 \text { followed by constant } 3.5) ; \text { treated water ave. }=5.61 \text { (constant) }\end{array}$ \\
\hline$\overline{E h}$ & - & $\begin{array}{l}\text { Media solution ave. }=255 \text { (apparently increasing over time }(120 \text { to } 350) \text {; } \\
\text { groundwater ave. }=330 \text { (greatest the first day }(382) \text { and fairly constant the rest of } \\
\text { the test constant over time }(\sim 320)) ; \text { treated water ave. }=182 \text { (increasing over time } \\
(108--200) \text { ) }\end{array}$ \\
\hline
\end{tabular}




\section{Table 26. Preliminary Data Observations Field Run \#3 Train \#2 Limestone \& Phosphate Rock \& Potassium Phosphate Solution:}

\begin{tabular}{|c|c|c|}
\hline Parameter & $\begin{array}{l}\text { Average } \\
\text { Change }\end{array}$ & Trend Comments \\
\hline$\overline{\mathrm{Al}}$ & $99 \%$ removal & $\begin{array}{l}\text { Constant \% removal ; Conc. in groundwater greatest the first } 2 \text { days }(\sim 150 \mathrm{ppm}) \\
\text { and fairly constant the rest of the test }(\sim 110 \mathrm{ppm}) \text {; Conc. in media solution below } \\
\text { detection }(<0.301 \mathrm{ppm}) \text {; Conc. in treated water below detection throughout the test } \\
(<0.301 \mathrm{ppm})\end{array}$ \\
\hline $\mathrm{Ca}$ & $83 \%$ addition & $\begin{array}{l}\text { \% addition highest first day }(333 \%) \text {; Conc. in groundwater greatest the first } 2 \text { days } \\
(\sim 104 \mathrm{ppm}) \text { and fairly constant the rest of the test }(\sim 67 \mathrm{ppm}) \text {; Conc. in media } \\
\text { solution fairly constant }(2.4 \mathrm{ppm}) \text {; Conc. in treated water greatest the first day }(\sim 228 \\
\mathrm{ppm}) \text { and variable around } 42 \mathrm{ppm} \text { the rest of the test }\end{array}$ \\
\hline $\mathrm{Cr}$ & $95 \%$ removal & $\begin{array}{l}\text { \% removal was lowest the last week }(81 \%) \text {; Conc. in groundwater increased with } \\
\text { time }(8-14 \mathrm{ppm}) \text {; Conc. in media solution below detection }(<0.060 \mathrm{ppm}) \text {; Conc. in } \\
\text { treated water appears to increase over time }(<0.060 \text { to } 1.232 \mathrm{ppm})\end{array}$ \\
\hline $\mathrm{Fe}$ & $69 \%$ removal & $\begin{array}{l}\text { Fairly constant \% removal except for last week at } 38 \% \text {; Conc. in groundwater } \\
\text { greatest the first } 2 \text { days }(\sim 190 \mathrm{ppm}) \text { and fairly constant the rest of the test }(\sim 80 \\
\text { ppm); Conc. in media solution increased to above detection the last two weeks of } \\
\text { the test }(<0.046-0.293) \text {; Conc. in treated water fairly constant ( } 17 \mathrm{ppm})\end{array}$ \\
\hline $\mathrm{Mg}$ & - & $\begin{array}{l}\text { Appears to be initially added followed by removal ( } 74 \% \text { addition first day to } 19 \% \\
\text { average removal the rest of the test); Conc. in groundwater greatest the first } 2 \text { days } \\
(\sim 60 \mathrm{ppm}) \text { and fairly constant the rest of the test }(\sim 37 \mathrm{ppm}) \text {; Conc. in media } \\
\text { solution increased the last two weeks of the test }(0.2 \text { to } 2.8 \mathrm{ppm}) \text {; Conc. in treated } \\
\text { water greatest the first } 2 \text { days }(-37 \mathrm{ppm}) \text { and fairly constant the rest of the test }(\sim 15 \\
\text { ppm) }\end{array}$ \\
\hline $\mathrm{Mn}$ & $47 \%$ removal & $\begin{array}{l}\text { Fairly constant } \% \text { removal except for last week at } 16 \% \text {; Conc. in groundwater } \\
\text { greatest the first } 2 \text { days }(-10 \mathrm{ppm}) \text { and fairly constant the rest of the test }(6-7 \\
\text { ppm); Conc. in media solution increased to above detection the last two weeks of } \\
\text { the test }(<0.028-0.093 \mathrm{ppm}) ; \text { Conc. in treated water fairly constant }(2.0 \mathrm{ppm})\end{array}$ \\
\hline $\mathrm{Ni}$ & - & $\begin{array}{l}\text { Have both \% removals and \% additions in no discernable pattern; Conc. in } \\
\text { groundwater appeared to decrease with time }(8-2 \mathrm{ppm}) \text {; Conc. in media solution } \\
\text { below detection }(<0.097 \mathrm{ppm}) \text {; Conc. in treated water decreased with time }(6.5-0.8 \\
\mathrm{ppm})\end{array}$ \\
\hline$P$ & $\begin{array}{l}-33,200 \% \\
\text { addition }\end{array}$ & $\begin{array}{l}\% \text { addition highest the first day and last week }(\sim 48,000 \%) \text { and fairly constant in } \\
\text { between }(\sim 26,000 \%) ; \text { Conc. in groundwater fairly constant }(0.37 \mathrm{ppm}) ; \text { Conc. in } \\
\text { media solution constant ( } 235 \mathrm{ppm}) \text {; Conc. in treated water fairly constant ( } 68 \mathrm{ppm})\end{array}$ \\
\hline $\mathrm{Si}$ & $8 \%$ removal & $\begin{array}{l}\text { Variable \% removal predominately; Conc. in groundwater constant }(78 \mathrm{ppm}) \text {; Conc. } \\
\text { in media solution fairly constant }(5.6 \mathrm{ppm}) \text {; Conc. in treated water constant ( } 36 \\
\mathrm{ppm})\end{array}$ \\
\hline Sulfate & $27 \%$ removal & $\begin{array}{l}\text { Fairly consistent \% removal; Conc. in groundwater greatest the first } 2 \text { days }(\sim 2400) \\
\text { and fairly constant the rest of the test }(-1275 \mathrm{ppm}) ; \text { Data for Conc. in media } \\
\text { solution suspect; Conc. in treated water greatest the first } 2 \text { days }(\sim 900 \mathrm{ppm}) \text { and } \\
\text { fairly constant the rest of the test }(\sim 640 \mathrm{ppm})\end{array}$ \\
\hline $\mathrm{pH}$ & - & $\begin{array}{l}\text { Media solution ave. }=7.92(8.45 \text { by omitting the lowest recorded } \mathrm{pH} \text {; fairly } \\
\text { constant); groundwater ave. }=3.23 \text { (initial two day's groundwater } \mathrm{pHs} \text { somewhat } \\
\text { low: } \sim 2.75 \text { followed by constant } 3.5 \text { ); treated water ave }=5.80 \text { (constant) }\end{array}$ \\
\hline $\mathrm{Eh}$ & - & $\begin{array}{l}\text { Media solution ave. }=255 \text { (apparently increasing over time }(120 \text { to } 350) \text {; } \\
\text { groundwater ave. }=330 \text { (greatest the first day }(382) \text { and fairly constant the rest of } \\
\text { the test constant over time }(-320) \text { ); treated water ave. }=149 \text { (increasing over time } \\
(66-180) \text { ) }\end{array}$ \\
\hline
\end{tabular}




\subsection{Laboratory Test \#1}

\subsubsection{Laboratory Observations}

\subsubsection{Reaction}

Precipitation was immediately visible in vials containing DCB $21 \mathrm{~A}$ groundwater with potassium phosphate dibasic and sodium phosphate dibasic combinations. Precipitation was also immediately visible in vials containing DCB 10 groundwater with potassium phosphate dibasic and sodium phosphate dibasic combinations. Potassium Phosphate 6.0+DCB-10 appears to have 2 layers of solids in the centrifuge tube. No precipitation reaction was visible in vials containing groundwater from DCB- $4 A$.

\subsubsection{Sediment}

Where precipitation occurred, the sediment was a white gelatinous substance. The following table represents the sediment results for this laboratory run:

Table 27. Laboratory Observations Lab Test \#1

\begin{tabular}{|l|c|l|l|l|}
\hline \multicolumn{2}{|c|}{ Sample ID } & \multicolumn{1}{|c|}{ ml sediment } & Other \\
\hline Potassium Phosphate 18.75 & + & DCB-21A & 7.5 & Slightly orange \\
\hline Potassium Phosphate 12.5 & + & DCB-21A & 10 & \\
\hline Potassium Phosphate 6.25 & + & DCB-21A & 10 & \\
\hline Potassium Phosphate 6.0 & + & DCB-10 & 16.25 & $\begin{array}{l}\text { More fluid, white, still } \\
\text { settling }\end{array}$ \\
\hline Potassium Phosphate 4.0 & + & DCB-10 & 12.5 & More fluid, white \\
\hline Potassium Phosphate 2.0 & + & DCB-10 & 5 & More fluid, white \\
\hline Potassium Phosphate 1.8 & + & DCB-4A & 0 & \\
\hline Potassium Phosphate 1.2 & + & DCB-4A & 0 & \\
\hline Potassium Phosphate 0.6 & + & DCB-4A & 0 & \\
\hline Sodium Phosphate 10.2 & + & DCB-21A & 8.25 & More fluid, white \\
\hline Sodium Phosphate 3.2 & + & DCB-10 & 5 & Still settling \\
\hline Sodium Phosphate 1.0 & + & DCB-4A & 0 & Still settling \\
\hline Phosphate Rock, pH 5.5 & + & DCB-21A & $\sim 2$ & \\
\hline Phosphate Rock, pH 5.5 & + & DCB-10 & $\sim 3$ & Still settling \\
\hline Phosphate Rock, pH 5.5 & + & DCB-4A & 0 & Not as opaque, still \\
\hline Phosphate Rock, pH 4.5 & + & DCB-21A & $\sim 4$ & \\
\hline Phosphate Rock, pH 4.5 & + & DCB-10 & $\sim 3$ & \\
\hline Phosphate Rock, pH 4.5 & + & DCB-4A & 0 & \\
\hline
\end{tabular}




\subsubsection{Data Observations Lab Test \#1}

Figures 28-37 depict the primary contaminants (aluminum, chromium, iron, and nickel) during this campaign. Preliminary observations are summarized below and shown in Tables 28-29.

Potassium Phosphate

In general, the greatest percentage removals occurred with higher concentrations of potassium phosphate for DCB-21A and DCB-10 groundwaters. The potassium phosphate solutions did not increase the $\mathrm{pH}$ of the DCB-10 groundwater as much as the DCB-21A groundwater. This may possibly be the reason for the decreased efficiencies associated with DCB-10 groundwater treatment. Overall, significant removals of aluminum, calcium, chromium, iron, magnesium, manganese, and nickel were observed using the potassium phosphate solution.

\section{Sodium Phosphate}

Overall, significant removals of aluminum, calcium, iron, magnesium, manganese, and nickel occurred with the potassium phosphate solution. There was no chromium in the DCB-21A groundwater to evaluate the sodium phosphate treatments.

\section{Phosphate Rock}

Generally, there was no significant treatment of any constituent except for iron at a reduction of $\sim 37 \%$. 
Figure 28. Laboratory Test \#1

Aluminum Concentration \& DCB-21A

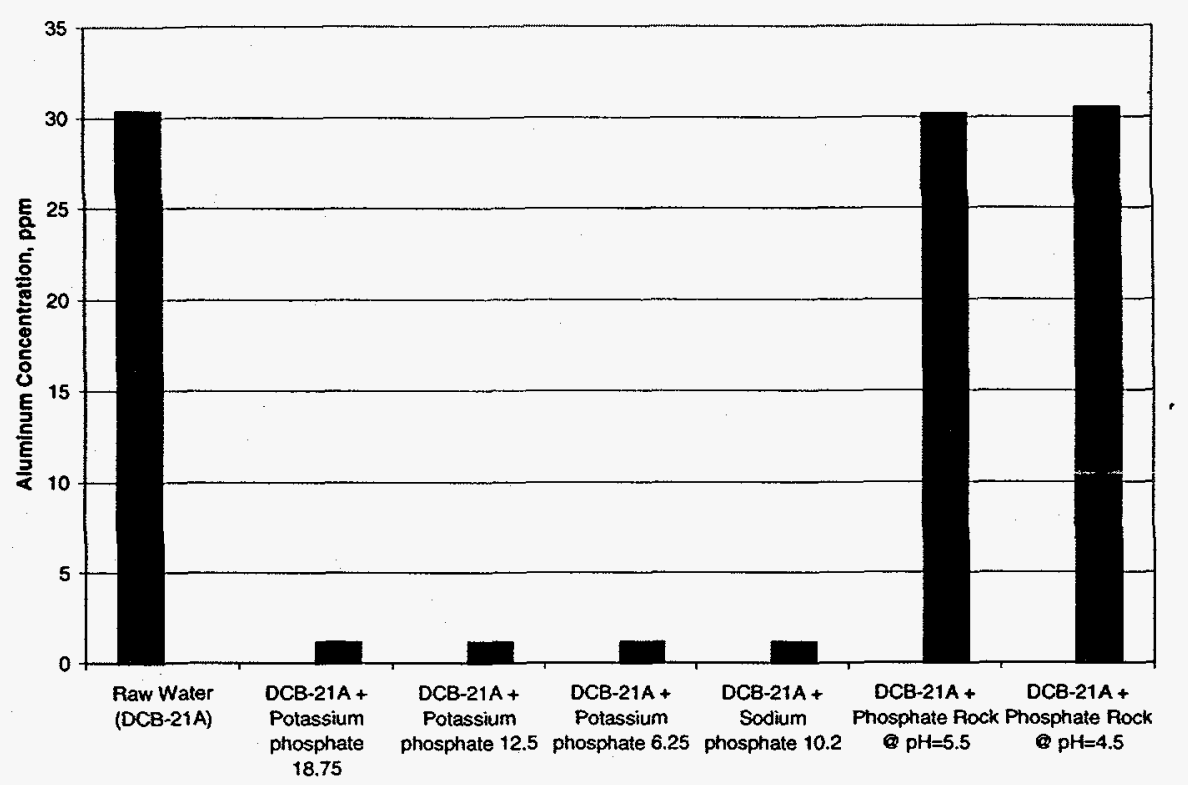

Figure 29. Laboratory Test \#1

Chromium Concentration \& DCB-21A

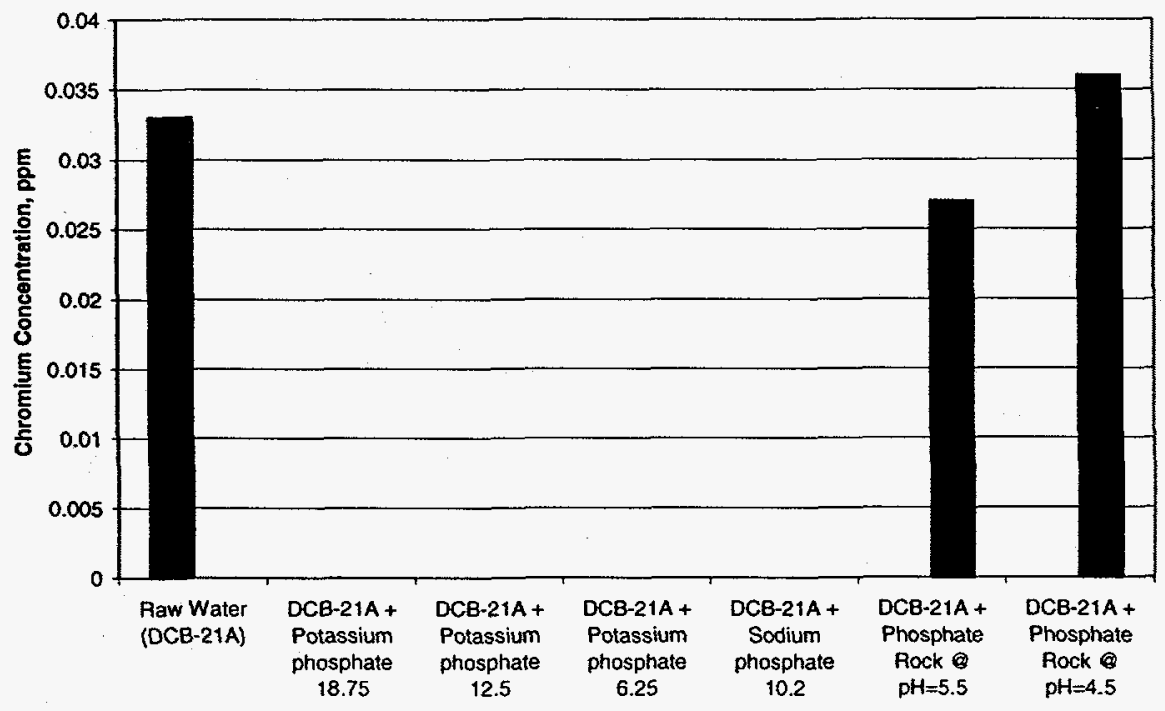


Figure 30. Laboratory Test \#1 Iron Concentration \& DCB-21A

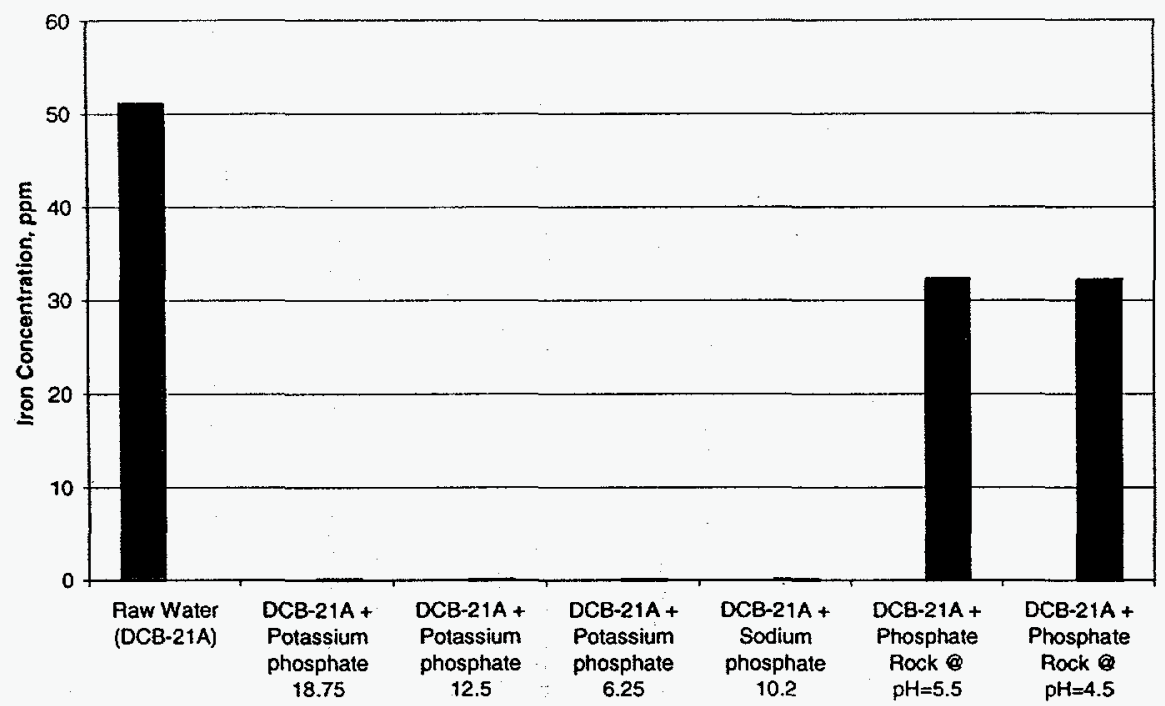

Figure 31. Laboratory Test \#1 Nickel Concentration \& DCB-21A

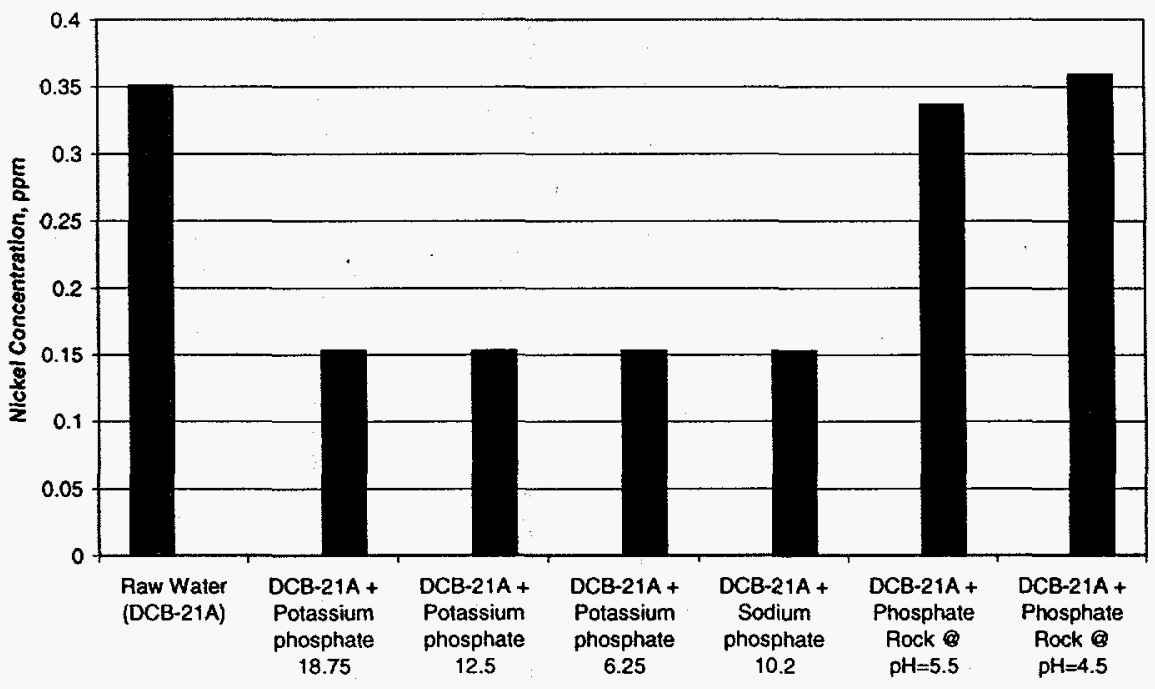


Figure 32. Laboratory Test \#1

Aluminum Concentration \& DCB-10

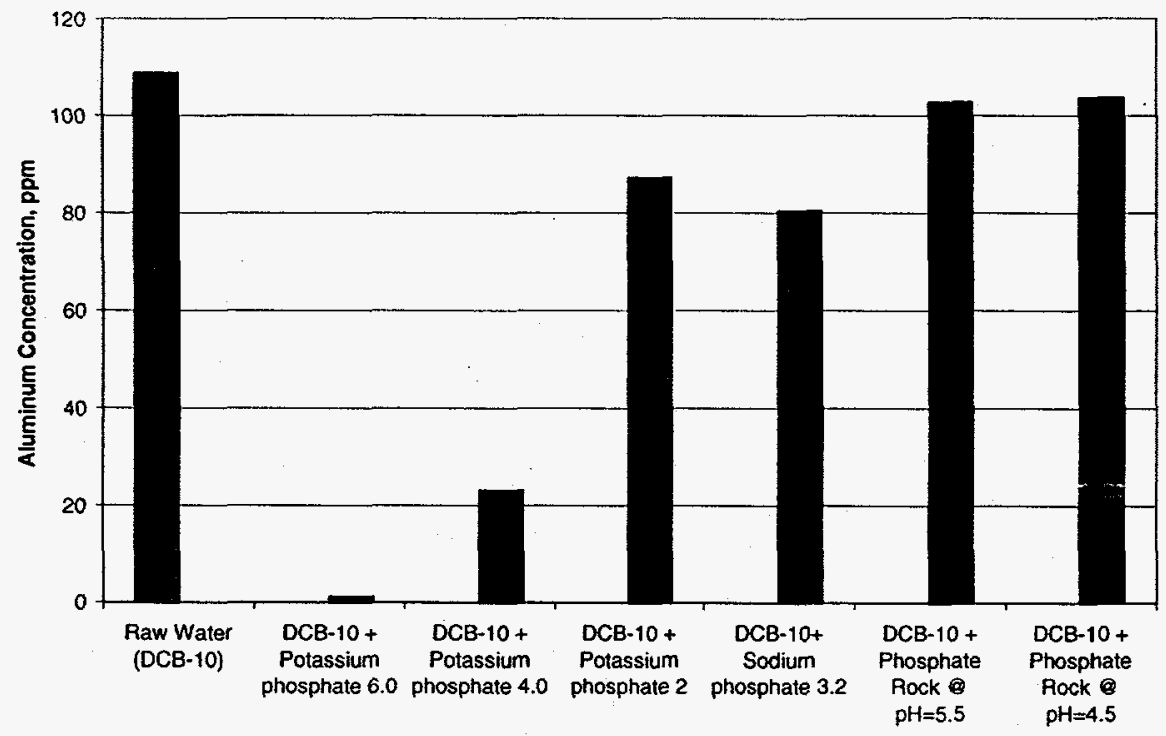

Figure 33. Laboratory Test \#1

Chromium Concentration \& DCB-10

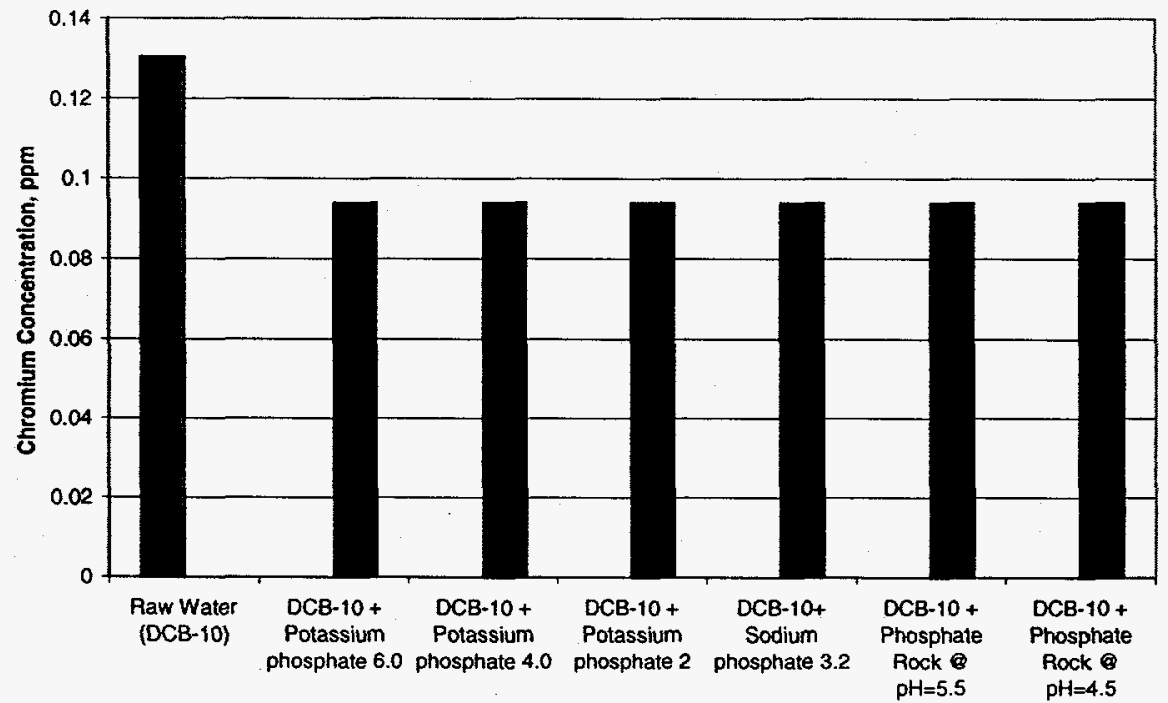


Figure 34. Laboratory Test \#1

Iron Concentration \& DCB-10

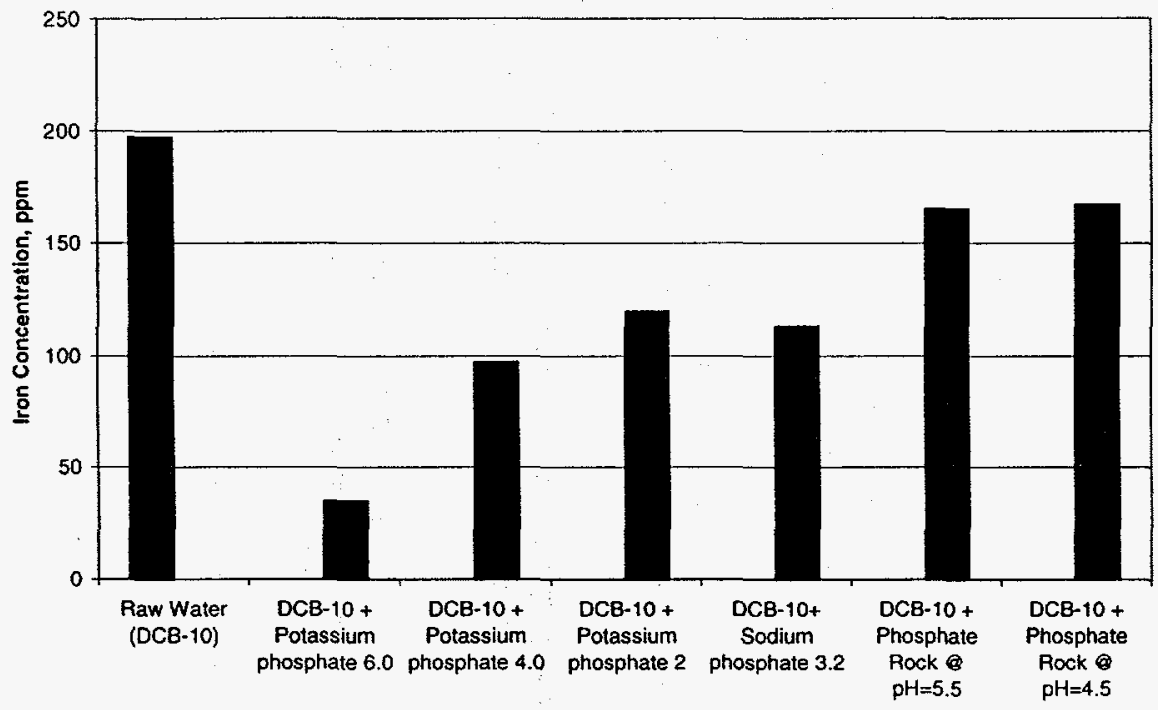

Figure 35. Laboratory Test \#1 Nickel Concentration \& DCB-10

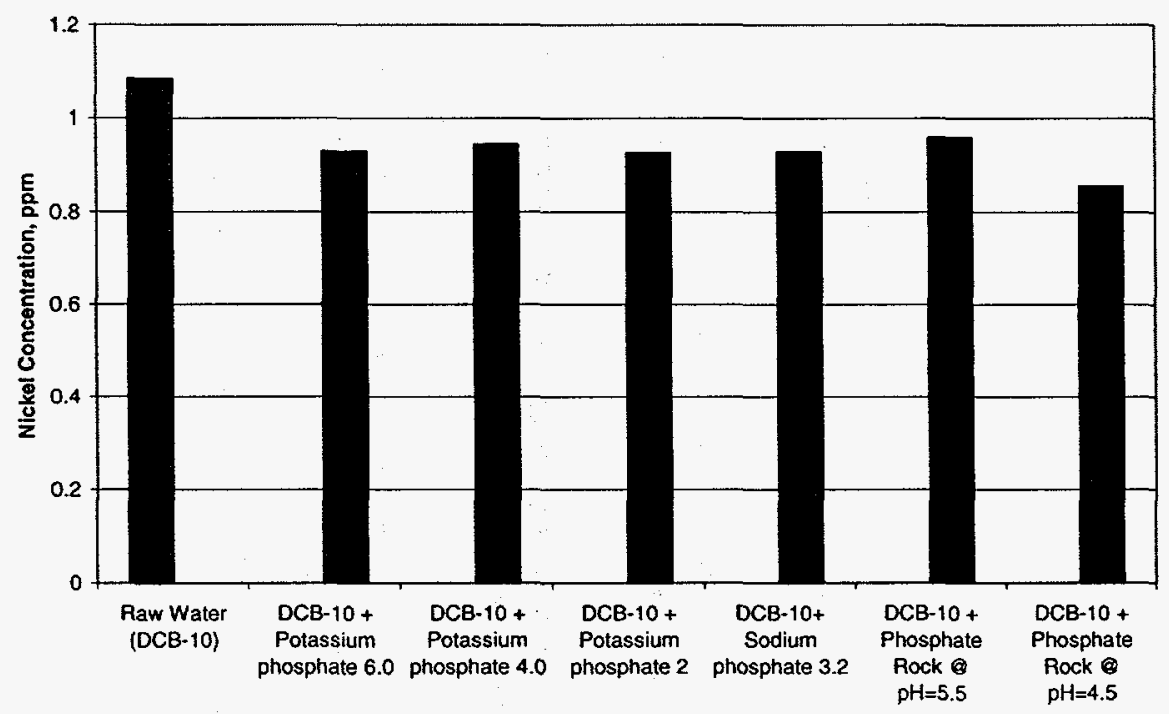


Figure 36. Laboratory Test \#1

Aluminum Concentration \& DCB-4A

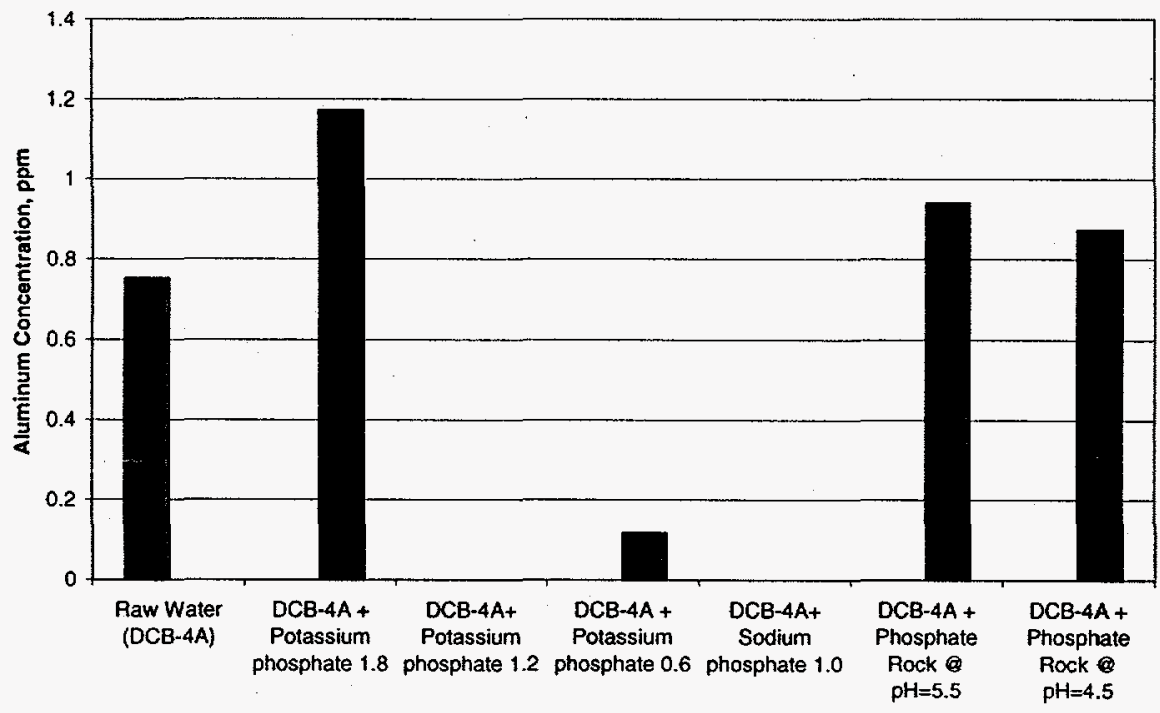

Figure 37. Laboratory Test \#1

Iron Concentration \& DCB-4A

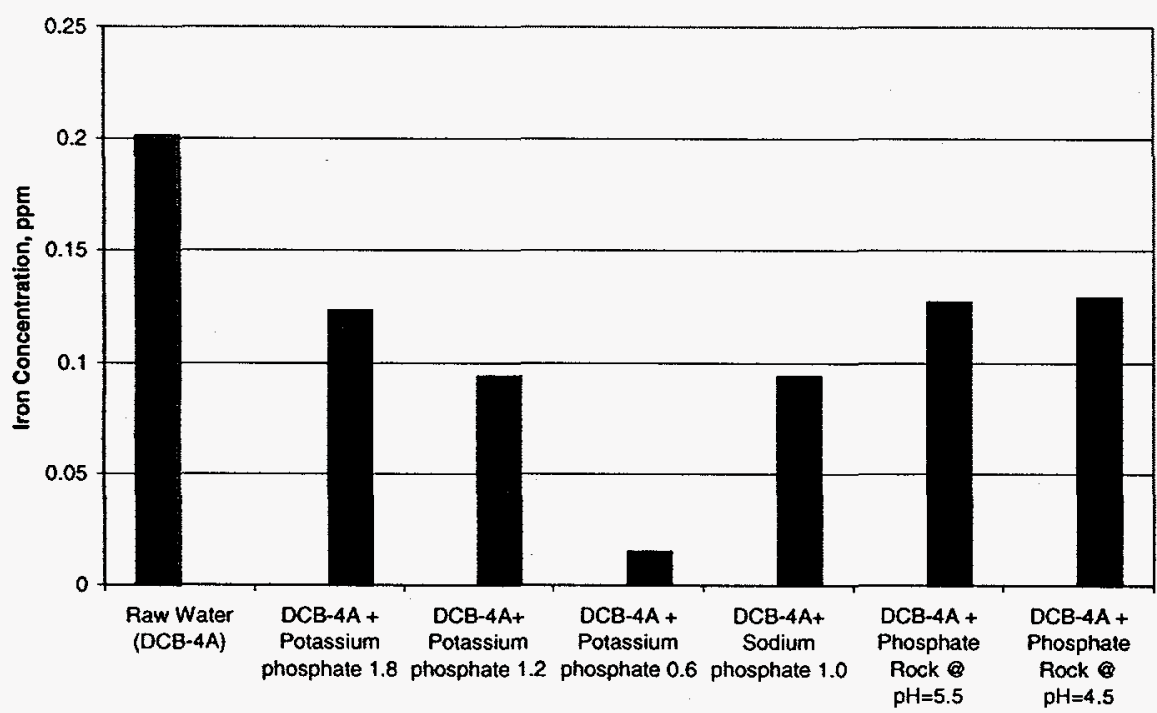


Table 28. Preliminary Observations Laboratory Test \#1 Potassium Phosphate Solution

\begin{tabular}{|c|c|c|c|c|c|c|}
\hline Parameter & DCB-21A & $\begin{array}{l}\text { Range of } \\
\% \text { Change }\end{array}$ & DCB-10 & $\begin{array}{l}\text { Range of } \\
\% \text { Change }\end{array}$ & DCB-4A & $\begin{array}{l}\text { Range of } \\
\% \text { Change }\end{array}$ \\
\hline $\mathrm{Al}$ & 61 & $96 \%$ R TBD & 218 & $99-20 \% \mathrm{R}$ & 1.5 & $\begin{array}{l}56 \% \mathrm{~A}-84 \% \\
\mathrm{R}\end{array}$ \\
\hline $\mathrm{Ca}$ & 74 & $85 \%-64 \% \mathrm{R}$ & 69 & $\begin{array}{l}13 \% \mathrm{R} \text { to } 2 \% \\
\mathrm{~A}\end{array}$ & 6.8 & $\begin{array}{l}24 \% \mathrm{~A}-38 \% \\
\mathrm{~A}-4 \% \mathrm{R}\end{array}$ \\
\hline $\mathrm{Cr}$ & 0.066 & - & 0.26 & $28 \%$ R TBD & $\mathrm{LD}$ & - \\
\hline $\mathrm{Fe}$ & 102 & $99 \%$ R TBD & 394 & $82-39 \% \mathrm{R}$ & 0.4 & $39-92 \% \mathrm{R}$ \\
\hline $\mathrm{Mg}$ & 43 & $47 \%-26 \% \mathrm{R}$ & 81 & $3 \% \mathrm{R}$ to little $\mathrm{A}$ & 4.3 & $10-0 \% \mathrm{R}$ \\
\hline $\mathrm{Mn}$ & 3 & $97 \%-92 \% \mathrm{R}$ & 8 & $21-2 \% \mathrm{R}$ & .1 & $10-26 \% \mathrm{R}$ \\
\hline $\mathrm{Ni}$ & 0.7 & $56 \%$ R TBD & 2 & $\sim 14 \% \mathrm{R}$ & $\mathrm{LD}$ & - \\
\hline $\mathrm{P}$ & 0.075 & $\begin{array}{l}3.8 \mathrm{M} \%-1 \mathrm{M} \% \\
\mathrm{~A}\end{array}$ & 0.29 & $\begin{array}{l}200 \mathrm{~K}-73 \mathrm{~K} \% \\
\mathrm{~A}\end{array}$ & 0.06 & $\begin{array}{l}500 \mathrm{~K}-150 \mathrm{~K} \% \\
\mathrm{~A}\end{array}$ \\
\hline $\mathrm{Si}$ & 69 & $2-9-5 \% \mathrm{~A}$ & 84 & - & 3.8 & $45-11 \% \mathrm{~A}$ \\
\hline Sulfate & 1725 & - & 4046 & - & 172 & $38-65 \% \mathrm{R}$ \\
\hline Phosphate & 2608 & $\begin{array}{l}332 \%-55 \%- \\
66 \% \mathrm{~A}\end{array}$ & 448 & $\begin{array}{l}223-337- \\
257 \% \mathrm{~A}\end{array}$ & 24 & $\begin{array}{l}3900-1170 \% \\
\text { A }\end{array}$ \\
\hline $\mathrm{pH}$ & 2.94 & $7.82-6.98$ & 2.6 & $3-4-3$ & 4.5 & $7.6-7.9-7.2$ \\
\hline Eh & 650 & $377-395$ & 639 & $454-375-504$ & 434 & $272-319^{\prime}$ \\
\hline
\end{tabular}

Notes:

- $\mathrm{R}=$ reduction; $\mathrm{A}=$ addition; $\mathrm{TBD}=$ to below detection; $\mathrm{K}=1000$ s $\mathrm{M}=$ millions

Table 29. Preliminary Observations Laboratory Test \#1 Sodium Phosphate Solution

\begin{tabular}{|l|l|l|l|l|l|l|}
\hline Parameter & DCB-21A & \% Change & DCB-10 & \% Change & DCB-4A & \% Change \\
\hline $\mathrm{Al}$ & 61 & $96 \% \mathrm{R}$ & 218 & $26 \% \mathrm{R}$ & 1.5 & $55 \% \mathrm{~A}$ \\
\hline $\mathrm{Ca}$ & 74 & $74 \% \mathrm{R}$ & 69 & $2 \% \mathrm{R}$ & 6.8 & $28 \% \mathrm{R}$ \\
\hline $\mathrm{Cr}$ & 0.066 & - & 0.26 & $28 \% \mathrm{R}$ & LD & \\
\hline $\mathrm{Fe}$ & 102 & $99 \% \mathrm{R}$ & 394 & $42 \% \mathrm{R}$ & 0.4 & $53 \% \mathrm{R}$ \\
\hline $\mathrm{Mg}$ & 43 & $32 \% \mathrm{R}$ & 81 & $2.5 \% \mathrm{R}$ & 4.3 & $5 \% \mathrm{~A}$ \\
\hline $\mathrm{Mn}$ & 3 & $93 \% \mathrm{R}$ & 8 & $5 \% \mathrm{R}$ & .1 & $24 \% \mathrm{R}$ \\
\hline $\mathrm{Ni}$ & 0.7 & $56 \% \mathrm{R}$ & 2 & $14 \% \mathrm{R}$ & $\mathrm{LD}$ & \\
\hline $\mathrm{P}$ & 0.075 & $1.2 \mathrm{M} \% \mathrm{~A}$ & 0.29 & $77.5 \mathrm{~K} \% \mathrm{~A}$ & 0.06 & $0.2 \mathrm{M} \% \mathrm{~A}$ \\
\hline $\mathrm{Si}$ & 69 & $9 \% \mathrm{~A}$ & 84 & $0.5 \% \mathrm{R}$ & 3.8 & $24 \% \mathrm{~A}$ \\
\hline Sulfate & 1725 & - & 4046 & $18 \% \mathrm{~A}$ & 172 & $57 \% \mathrm{R}$ \\
\hline $\mathrm{Phosphate}$ & 2608 & $30 \% \mathrm{~A}$ & 448 & $195 \% \mathrm{~A}$ & 24 & $1.7 \mathrm{~K} \mathrm{~A}$ \\
\hline $\mathrm{pH}$ & 2.94 & 7.1 & 2.6 & & 4.5 & \\
\hline Eh & 650 & 384 & 639 & & 434 & \\
\hline
\end{tabular}

Notes:

- $\quad \mathrm{R}=$ reduction; $\mathrm{A}=$ addition $; \mathrm{TBD}=$ to below detection $; \mathrm{K}=1000 \mathrm{~s} ; \mathrm{M}=$ millions 


\section{$5.5 \quad$ Laboratory Test \#2}

\subsubsection{Laboratory Observations}

\subsubsection{Reaction}

Precipitation was immediately visible in vials containing DCB $21 \mathrm{~A}$ groundwater with potassium phosphate dibasic combinations. Precipitation was also immediately visible in vials containing DCB 10 groundwater and potassium phosphate dibasic combinations. No precipitation was noted in vials containing calcium and ammonium phosphate.

\subsubsection{Sediment}

Where precipitation occurred, the sediment was a white gelatinous substance. The following table represents the sediment results for this laboratory run:

Table 30. Laboratory Observations Lab Test \#2

\begin{tabular}{|l|l|l|l|l|l|l|}
\hline \multicolumn{9}{|c|}{ Sample ID } & ml sediment & Other \\
\hline Ammonium Phosphate 14.22 & + & DCB-21A & & & 0 & \\
\hline Ammonium Phosphate 4.49 & + & DCB-10 & & & 0 & \\
\hline Ammonium Phosphate 1.33 & + & DCB-4A & & & 0 & \\
\hline Calcium Phosphate SAT'D & + & DCB-21A & & & & \\
\hline Calcium Phosphate SAT'D & + & DCB-10 & & & 0 & \\
\hline Calcium Phosphate SAT'D & + & DCB-4A & & & 0 & \\
\hline Potassium Phosphate 18.75 & + & DCB-21A & + & Phosphate Rock & 7.5 & \\
\hline Potassium Phosphate 6.0 & + & DCB-10 & + & Phosphate Rock & 10 & \\
\hline Potassium Phosphate 1.8 & + & DCB-4A & + & Phosphate Rock & 0 & \\
\hline Potassium Phosphate 18.75 & + & DCB-21A & + & Limestone & 6 & suspended fines \\
\hline Potassium Phosphate 6.0 & + & DCB-10 & + & Limestone & 10 & attached in areas \\
\hline Potassium Phosphate 1.8 & + & DCB-4A & + & Limestone & $<1$ & \\
\hline
\end{tabular}

\subsubsection{Data Observations Lab Test \#2}

Figures 38-43 depict the primary contaminants (aluminum, chromium, iron, and nickel) during this campaign. Preliminary observations are summarized below and shown in Table 31. 


\section{Analytical Summary}

Based upon these results, the ammonium phosphate appears to be by far the best treatment solution. The ammonium phosphate removes significant percentages of aluminum, calcium, chromium, iron, magnesium, manganese, nickel and silica. This is promising considering that ammonium phosphate may be available commercially as an agricultural grade fertilizer. If used, the ammonium phosphate would add ammonium and phosphate to the treated water. There are also concerns as to the validity of the results from this run. This experiment was conducted with groundwater, which had been stored refrigerated for approximately 30 days. Also, experimental results were delayed and not analyzed for approximately 60 days after the experiment was concluded. During this time, oxidation of the ferrous iron may have occurred. This factor may have contributed to the high treatment efficiencies.

The calcium phosphate may be the second best treatment media; however it may have solubility problems for its addition in an actual field condition.

The phosphate rock with potassium phosphate and the limestone with potassium phosphate appear essentially equivalent and possibly an improvement over potassium phosphate alone. The phosphate rock and potassium phosphate add significant calcium, magnesium and silica to the treated water in addition to potassium and phosphate. The limestone and potassium phosphate combination adds significant calcium and magnesium to the treated water in addition to potassium and phosphate. 
Figure 38. Laboratory Test \#2

Aluminum Concentration with Ammonium and Calcium Phosphates

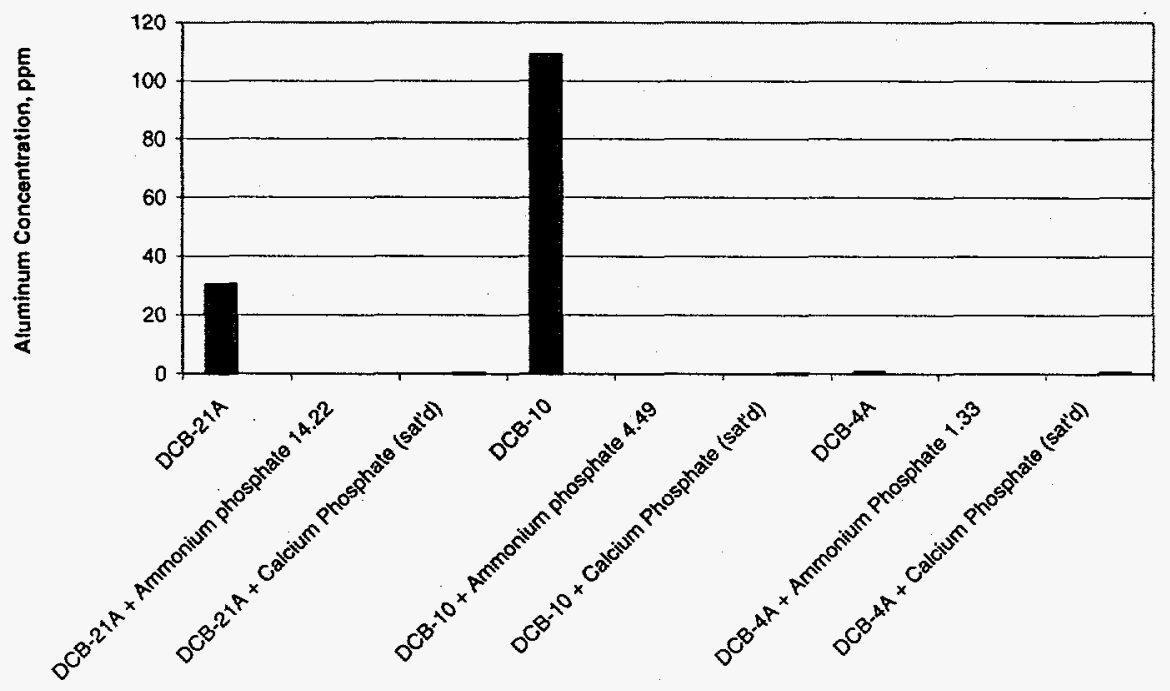

Figure 39. Laboratory Test \#2

Chromium Concentration with Ammonium and Calcium Phosphates

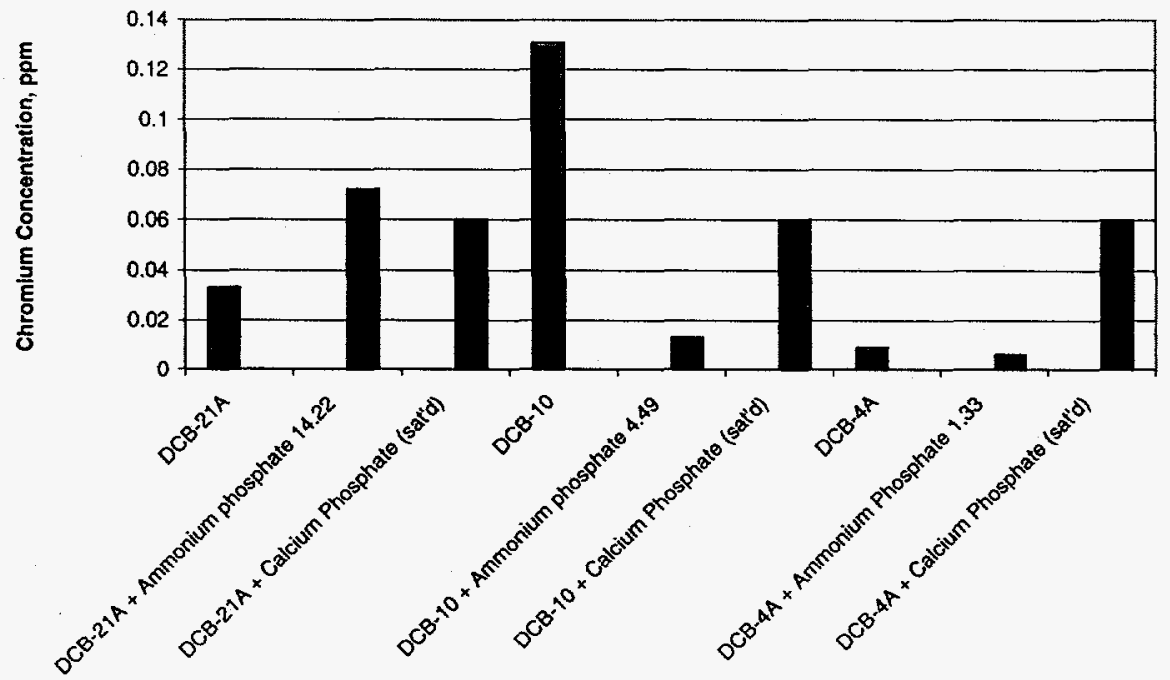


Figure 40. Laboratory Test \#2

Iron Concentration with Ammonium and Calcium Phosphates

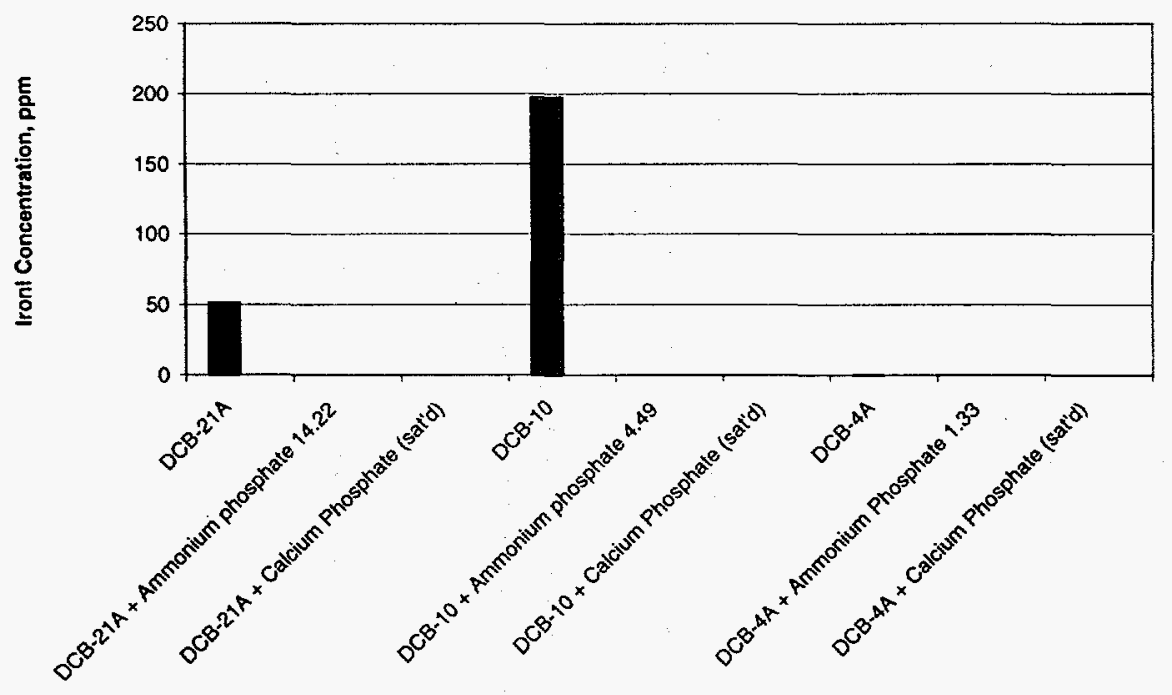

Figure 41. Laboratory Test \#2

Nickel Concentration with Ammonium and Calcium Phosphates

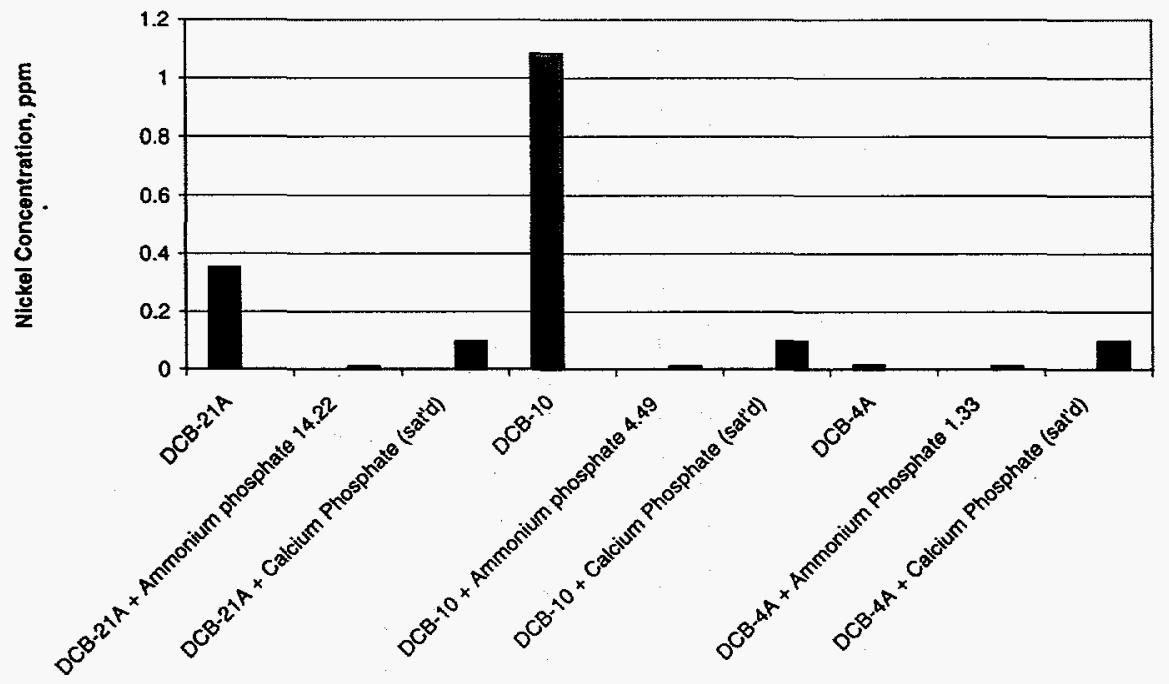


Figure 42. Laboratory Test \#2

Aluminum Concentration with Potassium Phosphate and a Reactive Matrix

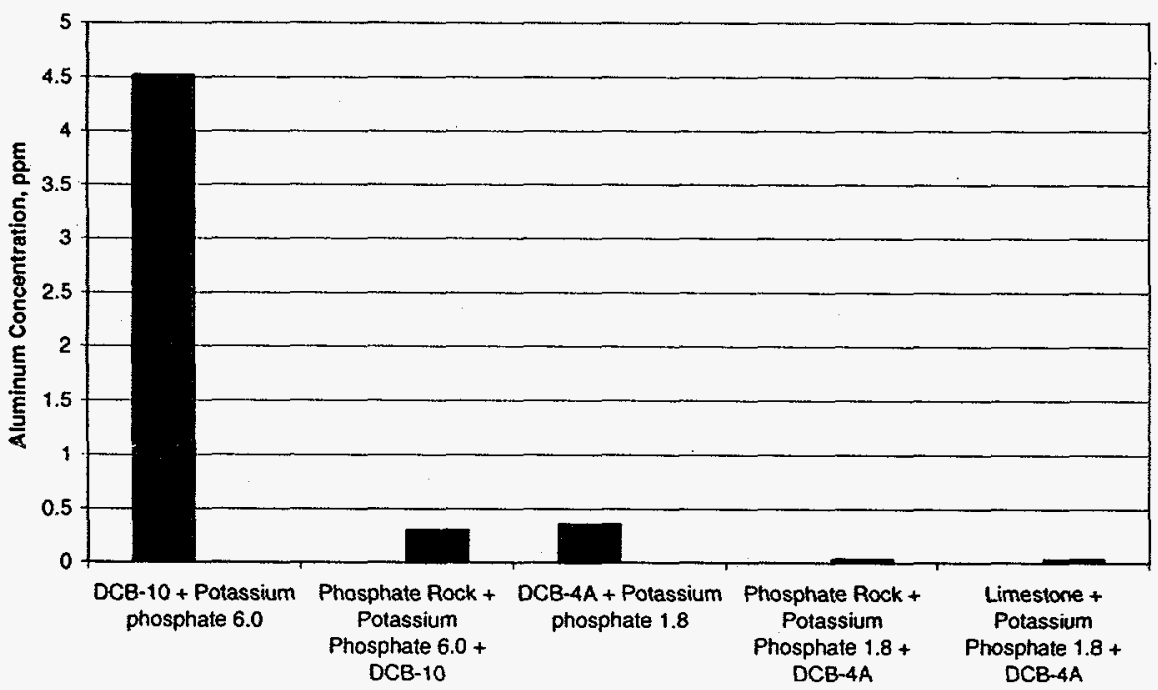

Figure 43. Laboratory Test \#2

Iron Concentration with Potassium Phosphate and a Reactive Matrix

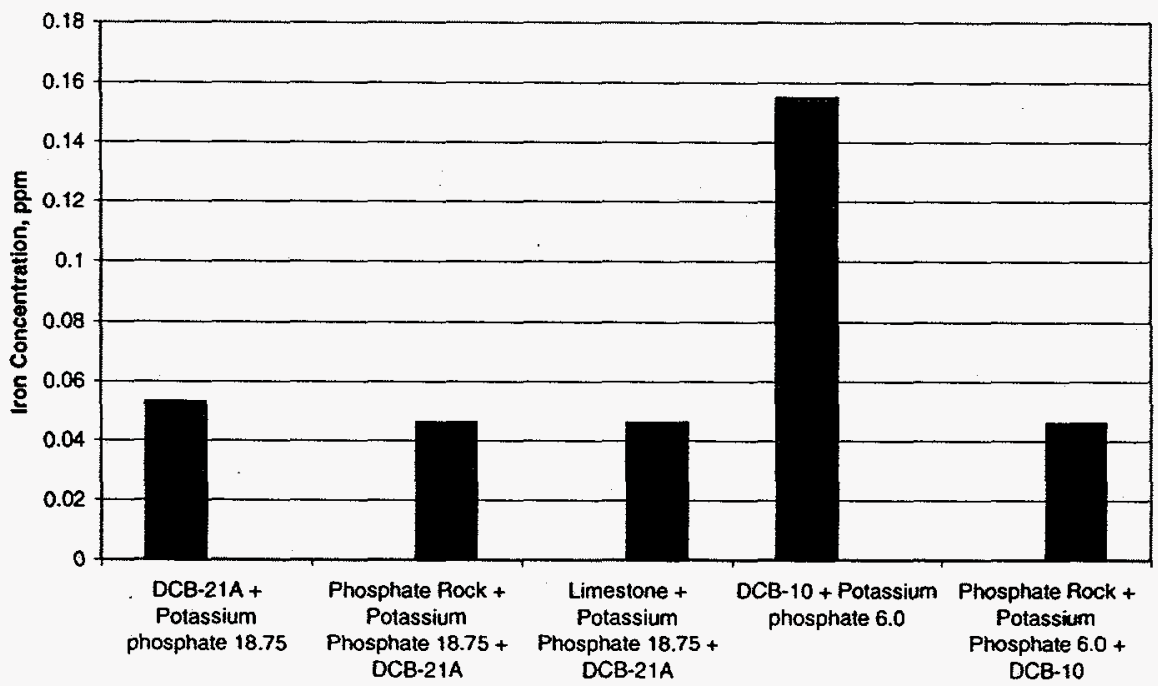


Table 31. Preliminary Observations Laboratory Test \#2

\begin{tabular}{|c|c|c|c|c|c|c|c|c|c|c|c|c|c|c|c|}
\hline Parameter & $\begin{array}{l}\text { Ammo } \\
\text { Phosp }\end{array}$ & $\begin{array}{l}\text { nium } \\
\text { ate }\end{array}$ & & Calcin & $n$ Phos & hate & Potass & $\mathrm{um} \mathrm{Phc}$ & sphate & $\begin{array}{l}\text { Phosph } \\
\text { Potass }\end{array}$ & $\begin{array}{l}\text { ate Ro } \\
\text { um Ph }\end{array}$ & $\begin{array}{l}\text { k \& } \\
\text { sphate }\end{array}$ & $\begin{array}{l}\text { Limes } \\
\text { Potass }\end{array}$ & $\begin{array}{l}\text { one \& } \\
\text { um Pho }\end{array}$ & sphate \\
\hline Well & $21 \mathrm{~A}$ & $10 \mathrm{D}$ & $4 \mathrm{~A}$ & $21 \mathrm{~A}$ & $10 \mathrm{D}$ & $4 \mathrm{~A}$ & $21 \mathrm{~A}$ & $10 \mathrm{D}$ & $4 \mathrm{~A}$ & $21 \mathrm{~A}$ & 100 & $4 \mathrm{~A}$ & $21 \mathrm{~A}$ & 100 & $4 \mathrm{~A}$ \\
\hline $\mathrm{Al}$ & $99 \mathrm{R}$ & $99 \mathrm{R}$ & $96 \mathrm{R}$ & $99 \mathrm{R}$ & 99R & $31 \mathrm{R}$ & 99R & $95 \mathrm{R}$ & $52 \mathrm{R}$ & $99 \mathrm{R}$ & 99R & $96 \mathrm{R}$ & $99 \mathrm{R}$ & & $96 \mathrm{R}$ \\
\hline $\mathrm{Ca}$ & $97 \mathrm{R}$ & $98 \mathrm{R}$ & $12 \mathrm{R}$ & $\begin{array}{l}1.7 \mathrm{~K} \\
\mathrm{~A}\end{array}$ & $\begin{array}{l}1.8 \mathrm{~K} \\
\mathrm{~A}\end{array}$ & $\begin{array}{l}10 \mathrm{~K} \\
\mathrm{~A}\end{array}$ & $85 \mathrm{R}$ & - & $3 \mathrm{~A}$ & $79 \mathrm{R}$ & $53 \mathrm{~A}$ & $14 \mathrm{~A}$ & $38 \mathrm{R}$ & & $482 \mathrm{~A}$ \\
\hline $\mathrm{Cr}$ & - & $90 \mathrm{R}$ & $=$ & - & $54 \mathrm{R}$ & - & - & $31 \mathrm{R}$ & - & - & $54 \mathrm{R}$ & - & $=$ & & - \\
\hline $\mathrm{Fe}$ & 99R & $99 \mathrm{R}$ & $97 \mathrm{R}$ & 99R & 99R & $77 \mathrm{R}$ & 99R & 99R & $97 \mathrm{R}$ & $99 \mathrm{R}$ & $99 \mathrm{R}$ & 97R & $99 \mathrm{R}$ & & $97 \mathrm{R}$ \\
\hline $\mathrm{Mg}$ & 99R & 99R & - & 95R & 97R & $31 \mathrm{~A}$ & $48 \mathrm{R}$ & $7 \mathrm{R}$ & & $38 \mathrm{R}$ & $11 \mathrm{~A}$ & $50 \mathrm{~A}$ & $52 \mathrm{~A}$ & & $453 \mathrm{~A}$ \\
\hline Mn & $99 \mathrm{R}$ & 99R & $36 \mathrm{R}$ & 98R & $99 \mathrm{R}$ & $5.7 R$ & 95R & $9 \mathrm{R}$ & $18 \mathrm{R}$ & $96 \mathrm{R}$ & 26R & $77 \mathrm{R}$ & $98 \mathrm{R}$ & & 95R \\
\hline $\mathrm{Ni}$ & $97 \mathrm{R}$ & $99 \mathrm{R}$ & - & $72 \mathrm{R}$ & 91R & - & $85 \mathrm{R}$ & $16 \mathrm{R}$ & - & $72 \mathrm{R}$ & $31 \mathrm{R}$ & - & $72 \mathrm{R}$ & & - \\
\hline $\mathbf{P}$ & $\begin{array}{l}8.1 \mathrm{M} \\
\mathrm{A}\end{array}$ & $\begin{array}{l}645 \mathrm{~K} \\
\mathrm{~A}\end{array}$ & $\begin{array}{l}463 \mathrm{~K} \\
\mathrm{~A} \\
\end{array}$ & $\begin{array}{l}2.6 \mathrm{M} \\
\mathrm{A} \\
\end{array}$ & $\begin{array}{l}685 \mathrm{~K} \\
\mathrm{~A} \\
\end{array}$ & $1.7 \mathrm{M}$ & $\begin{array}{l}3.7 \mathrm{M} \\
\mathrm{A} \\
\end{array}$ & $\begin{array}{l}162 \mathrm{~K} \\
\mathrm{~A} \\
\end{array}$ & $\begin{array}{l}451 \mathrm{~K} \\
\mathrm{~A} \\
\end{array}$ & $\begin{array}{l}3.3 \mathrm{M} \\
\mathrm{A} \\
\end{array}$ & $\begin{array}{l}152 \mathrm{~K} \\
\mathrm{~A}\end{array}$ & $360 \mathrm{~A}$ & $\begin{array}{l}2.6 \mathrm{M} \\
\mathrm{A} \\
\end{array}$ & & $\begin{array}{l}281 \mathrm{~K} \\
\mathrm{~A}\end{array}$ \\
\hline Si & $99 \mathrm{R}$ & $99 \mathrm{R}$ & - & $97 \mathrm{R}$ & $97 \mathrm{R}$ & $43 \mathrm{~A}$ & $3 R$ & $2 \mathrm{R}$ & - & $3.5 \mathrm{~A}$ & $3.6 \mathrm{R}$ & $65 \mathrm{~A}$ & $9 \mathrm{R}$ & & $78 \mathrm{~A}$ \\
\hline Sulfate & - & - & - & - & - & - & - & $33 R$ & $42 \mathrm{R}$ & $24 \mathrm{~A}$ & $30 \mathrm{R}$ & $12 \mathrm{R}$ & - & & $38 \mathrm{R}$ \\
\hline Phosphate & $534 \mathrm{~A}$ & $=$ & $\begin{array}{l}11 \mathrm{~K} \\
\mathrm{~A}\end{array}$ & $115 \mathrm{~A}$ & $\begin{array}{l}1.3 \mathrm{~K} \\
\mathrm{~A}\end{array}$ & $\begin{array}{l}17.9 \\
\mathrm{KA} \\
\end{array}$ & $354 \mathrm{~A}$ & $305 \mathrm{~A}$ & $\begin{array}{l}3978 \\
\mathrm{~A}\end{array}$ & $266 \mathrm{~A}$ & $347 \mathrm{~A}$ & $\begin{array}{l}3270 \\
\mathrm{~A}\end{array}$ & - & & $\begin{array}{l}2506 \\
\mathrm{~A} \\
\end{array}$ \\
\hline $\mathrm{pH}$ & 8.27 & 8.21 & 7.94 & 3.64 & 3.65 & 3.78 & 7.76 & 3.97 & 7.73 & 7.74 & 4.3 & 7.59 & 7.59 & 6.8 & 7.34 \\
\hline Eh & 325.5 & 309.7 & 335.9 & 531.5 & 526.1 & 487.3 & 271.2 & 516.1 & 342.1 & 362.3 & 487.7 & 363.0 & 373.4 & 404.1 & 380.5 \\
\hline
\end{tabular}

\section{$5.6 \quad$ Laboratory Test \#3}

\subsubsection{Laboratory Observations}

\subsubsection{Reaction}

No apparent precipitation reactions like those in Laboratory Test \#1 were observed.

\subsubsection{Sediment}

All tubes with sediment seemed to be the same color as the matrix fines. The amount of sediment was determined by subtracting the initial solid volume from the final solid volume. The following table represents the sediment results for this laboratory run:

Table 32. Laboratory Observations Lab Test \#3

\begin{tabular}{|l|c|}
\hline \multicolumn{1}{|c|}{ Sample ID } & ml sediment \\
\hline Limestone, 10\%, 5 minute mix & $\sim 1.5$ \\
\hline Limestone, 10\%, 10 minute mix & $\sim 2$ \\
\hline Limestone, 20\%, 5 minute mix & 2.5 \\
\hline Limestone, 20\%, 10 minute mix & $\sim 3$ \\
\hline Phosphate Rock, 10\%, 5 minute mix & $<0.5$ \\
\hline Phosphate Rock, 10\%, 10 minute mix & $<1$ \\
\hline Phosphate Rock, 20\%, 5 minute mix & $<1$ \\
\hline Phosphate Rock, 20\%, 10 minute mix & $\sim 2$ \\
\hline Limestone/Phosphate Rock, 20\%, 5 minute mix & $\sim 3$ \\
\hline Limestone/Phosphate Rock, 20\%, 10 minute mix & $\sim 3$ \\
\hline
\end{tabular}




\subsubsection{Data Observations Lab Test \#3}

Figures 44-47 depict the primary contaminants (aluminum, chromium, iron, and nickel) during this campaign. Preliminary observations are summarized below and shown in Table 33 .

In general, the best removal was obtained with the $20 \%$ limestone powder additions. It appeared that a 72-hour hold time with $20 \%$ limestone provided the best reduction in iron and nickel. There does not appear to be any significant difference between the $20 \%$ limestone and the $20 \%$ limestone/phosphate rock treatments.

Overall, the phosphate rock powder provided the least treatment.

Figure 44. Laboratory Test \#3

Aluminum Concentration with Limestone and/or Phosphate Rock

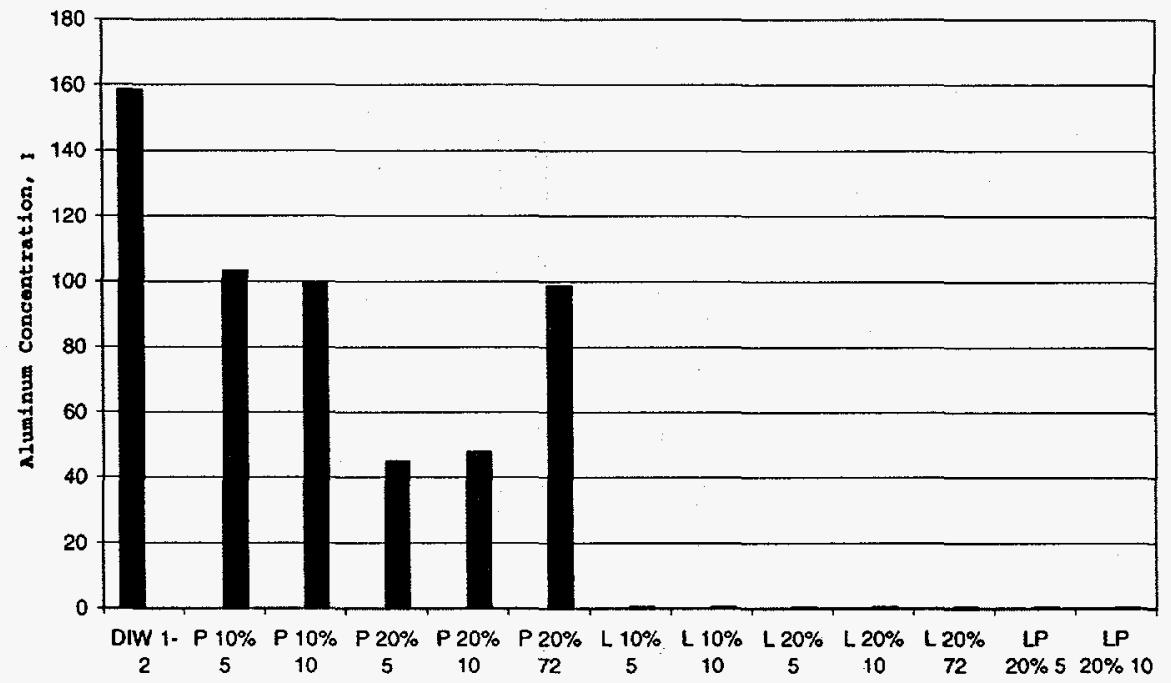


Figure 45. Laboratory Test \#3

Chromium Concentration with Limestone and/or Phosphate Rock

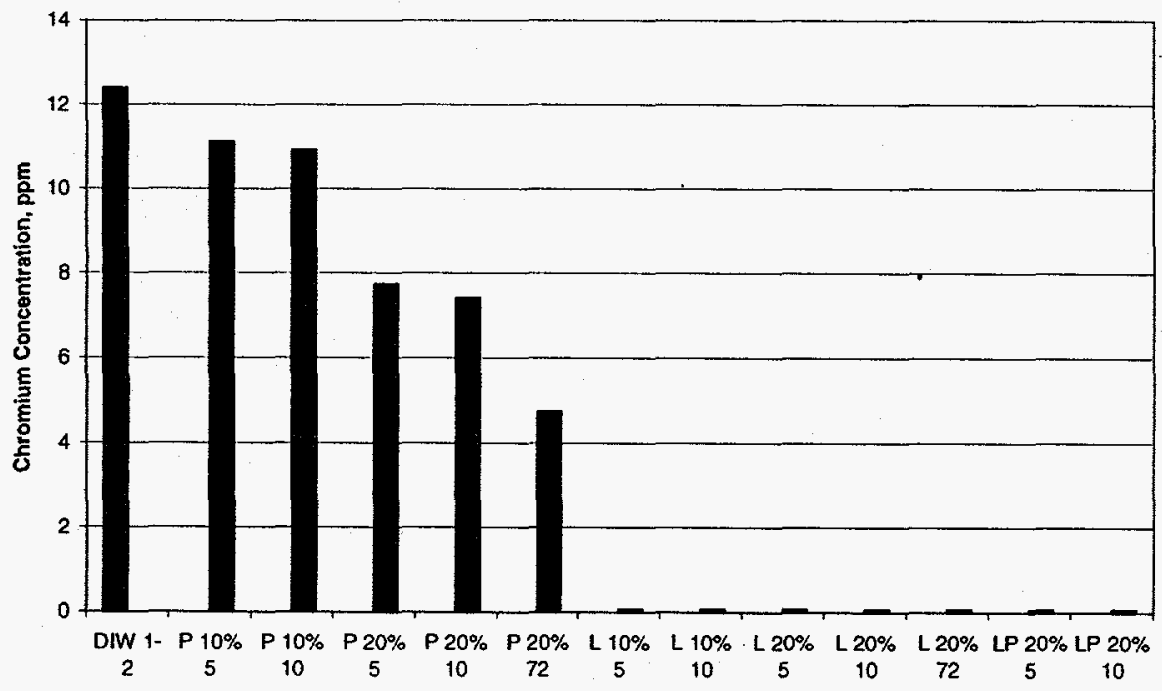

Figure 46. Laboratory Test \#3

Iron Concentration with Limestone and/or Phosphate Rock

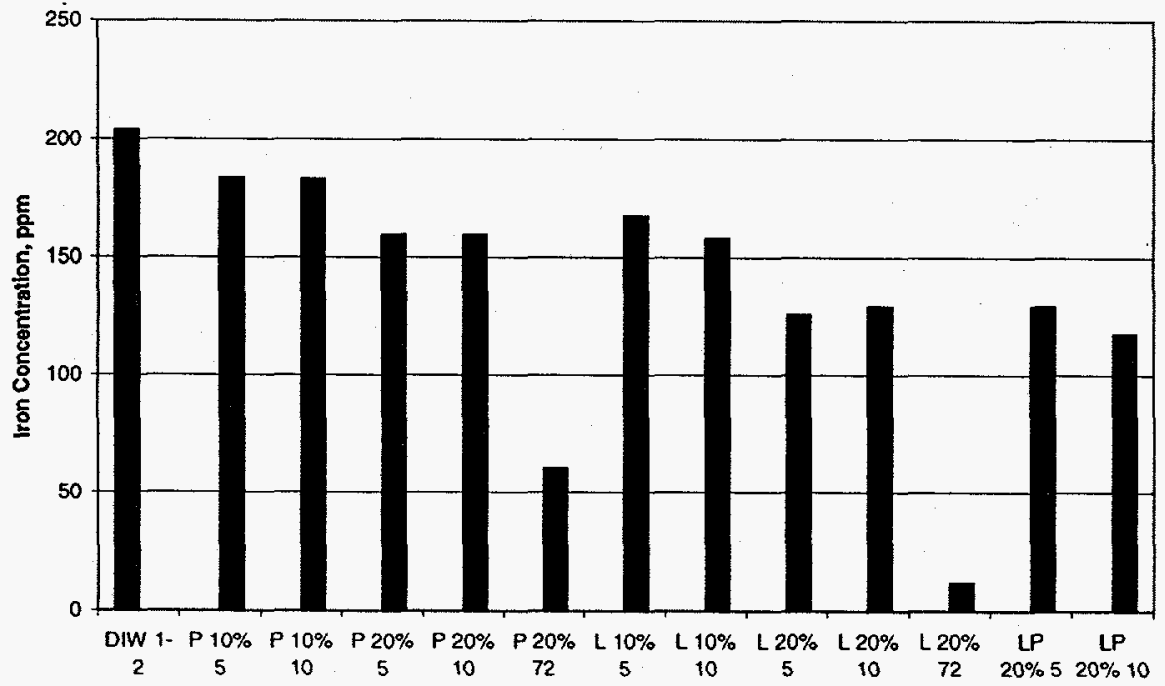


Figure 47. Laboratory Test \#3

Nickel Concentration with Limestone and/or Phosphate Rock

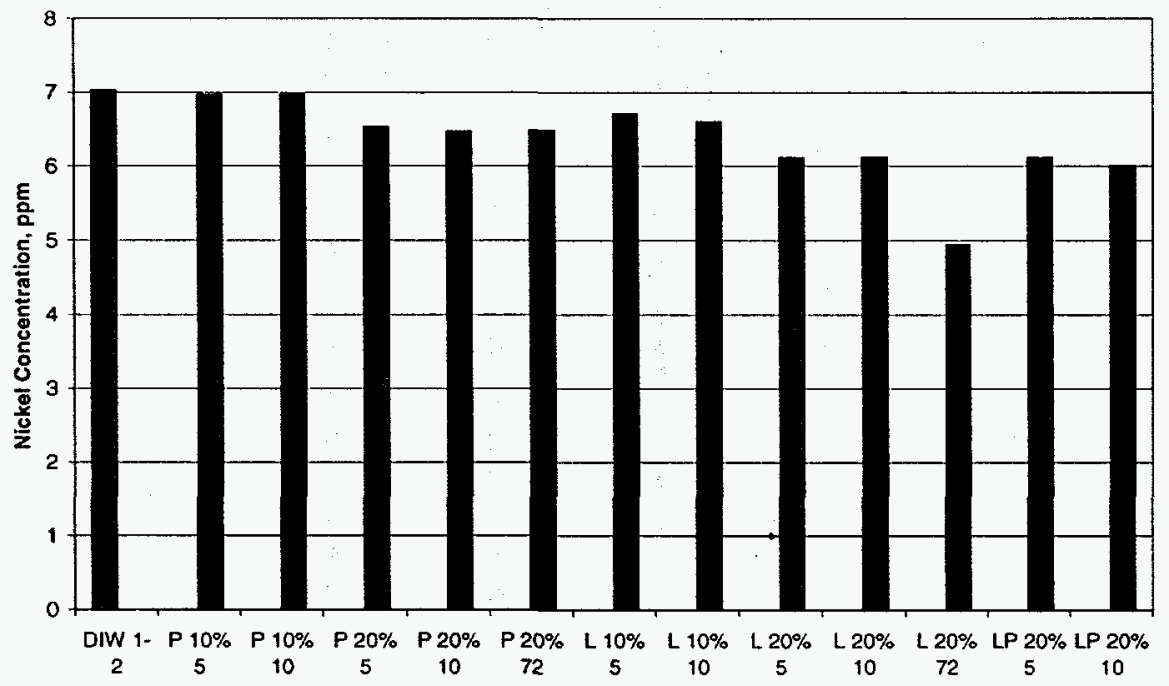

Table 33. Preliminary Observations Lab Test \#3

\begin{tabular}{|c|c|c|c|c|c|c|c|}
\hline DWW-1-2 & $\begin{array}{r}\text { Average } \\
\text { P } 10 \% 5 / 10 \\
\% \text { Change }\end{array}$ & $\begin{array}{r}\text { Average } \\
\text { P } 20 \% 5 / 10 \\
\% \text { Change }\end{array}$ & $0 \% 24$ & $\begin{array}{r}\text { Average } \\
\text { L } 10 \% 5 / 10 \\
\% \text { Change }\end{array}$ & $\begin{array}{r}\text { Average } \\
\text { L } 20 \% 5 / 10 \\
\% \text { Change }\end{array}$ & $\begin{array}{l}\% 24 \\
\text { hange }\end{array}$ & $\begin{array}{r}\text { Average } \\
\text { LP } 20 \% 5 / 10 \\
\% \text { Change }\end{array}$ \\
\hline 158.359 & -36.0 & -70.8 & -37.7 & -99.8 & -99.8 & -99.8 & -99.8 \\
\hline 103.257 & 195.6 & 289.6 & 218.3 & 495.4 & 525.9 & 573.8 & 515.6 \\
\hline 12.402 & -11.1 & -38.8 & -61.8 & -99.5 & -99.5 & -99.5 & -99.5 \\
\hline 203.871 & -10.2 & -21.9 & -70.4 & -20.3 & -37.6 & -94.1 & -39.6 \\
\hline 63.093 & 36.1 & 66.9 & 60.8 & 15.5 & 26.7 & 28.0 & 26.9 \\
\hline 10.153 & 1.9 & -2.8 & -4.6 & -9.0 & -21.9 & -31.1 & -20.9 \\
\hline 7.025 & -0.9 & -7.6 & -7.9 & -5.4 & -12.9 & -29.7 & -13.8 \\
\hline 0.273 & 5244.9 & 2152.7 & & & & & \\
\hline 80.727 & 3.1 & 3.8 & 3.7 & -32.3 & -38.0 & -61.8 & -37.2 \\
\hline 1924.835 & -2.9 & -1.8 & & -11.3 & -13.8 & & -9.8 \\
\hline 3.18 & 3.8 & 4.3 & NRT & 6.2 & 6.3 & NRT & 6.2 \\
\hline 363.1 & 368.9 & 344.4 & NRT & 165.1 & 148.6 & NRT & 172.6 \\
\hline
\end{tabular}




\subsection{Laboratory Test \#4}

\subsubsection{Laboratory Observations}

\subsubsection{Reaction}

No apparent precipitation reactions like those in Laboratory Test \#1 were observed.

\subsubsection{Sediment}

All tubes with sediment seemed to be the same color as the matrix fines. The amount of sediment was determined by subtracting the initial solid volume from the final solid volume. The following table represents the sediment results for this laboratory run:

\section{Table 34. Laboratory Observations Lab Test \#4}

\begin{tabular}{|l|c|c|}
\hline \multicolumn{1}{|c|}{ Sample ID } & ml sediment & Other \\
\hline Limestone, DIW-1-2 & 4.0 & \\
\hline Limestone, DCB 21A & 3.0 & \\
\hline Potassium phosphate, DIW-1-2 & 7.5 & Highly suspended \\
\hline Potassium phosphate, DCB 21A & 5.0 & Highly suspended \\
\hline
\end{tabular}

\subsubsection{Data Observations Lab Test \#4}

Figures 48-51 depict the primary contaminants (aluminum, chromium, iron, and nickel) during this campaign. Preliminary observations are summarized below and shown in Table 35.

Overall, the aeration and limestone treatment provided the best treatment for both DIW-1-2 and DCB-21A groundwater. The limestone reduced both chromium and nickel to a greater extent than the potassium phosphate solutions. In general, aeration and potassium phosphate performed very poorly on DCB-21A groundwater. 
Figure 48. Laboratory Test \#4 Aluminum Concentration with Aerated Wastewater

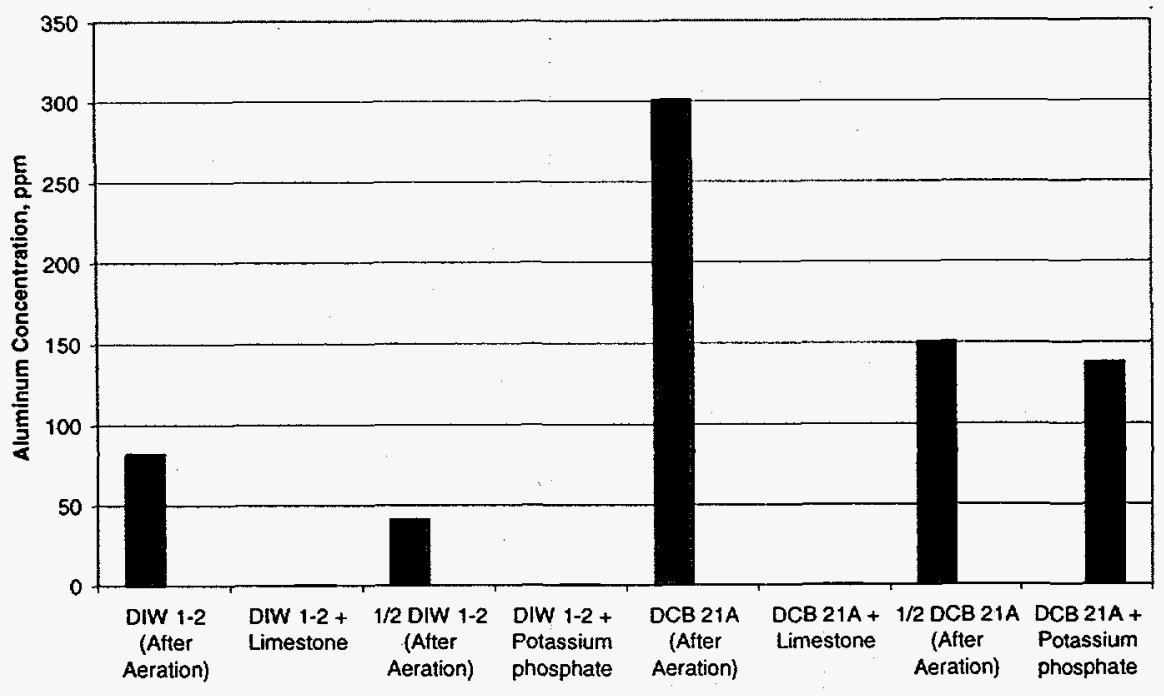

Figure 49. Laboratory Test \#4

Chromium Concentration with Aerated Wastewater

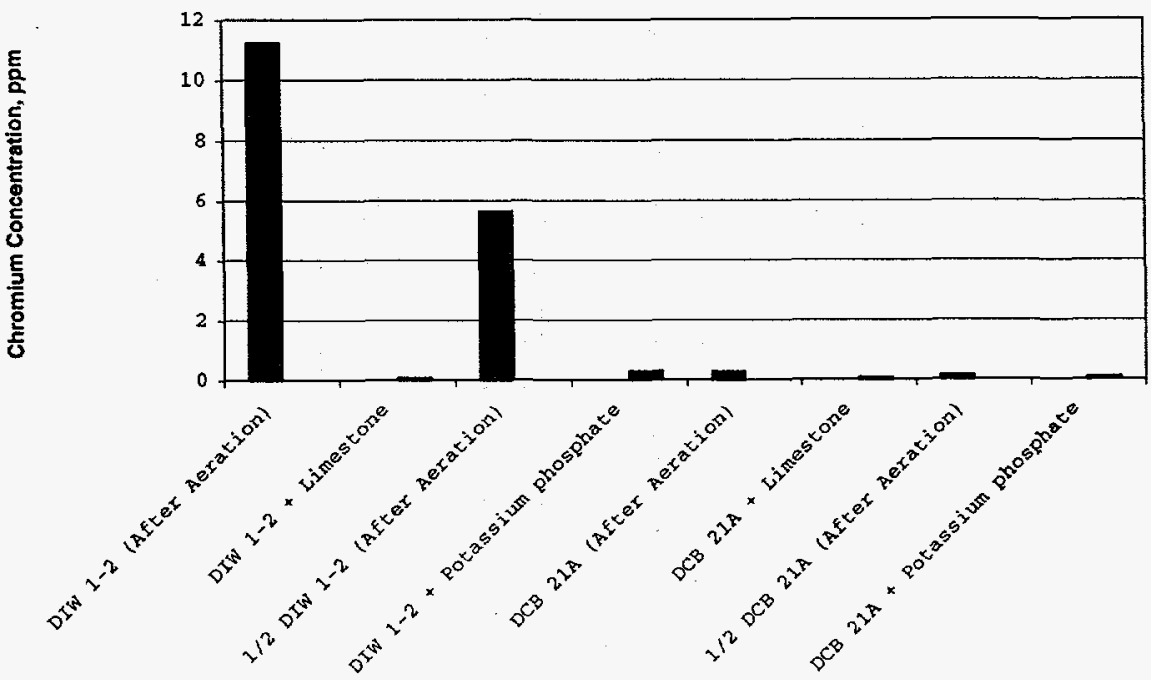


Figure 50, Laboratory Test 44

Iron Concentration with Aerated Wastewater

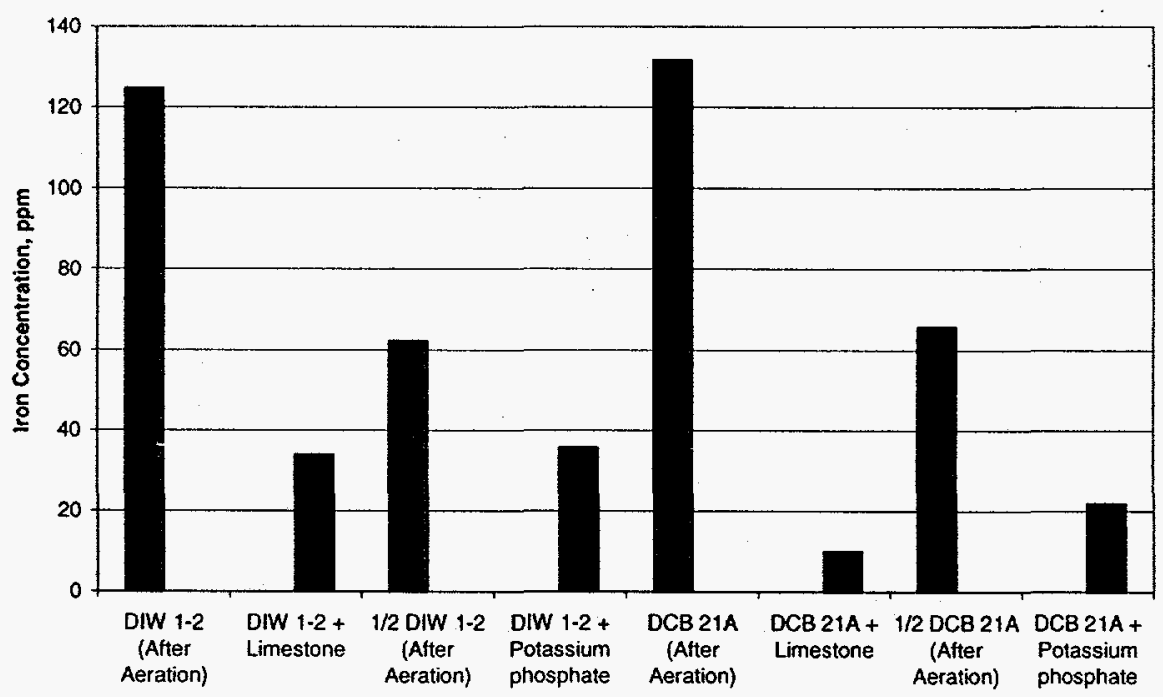

Figure 51. Laboratory Test $\$ 4$

Nickel Concentration with Aerated Wastwater

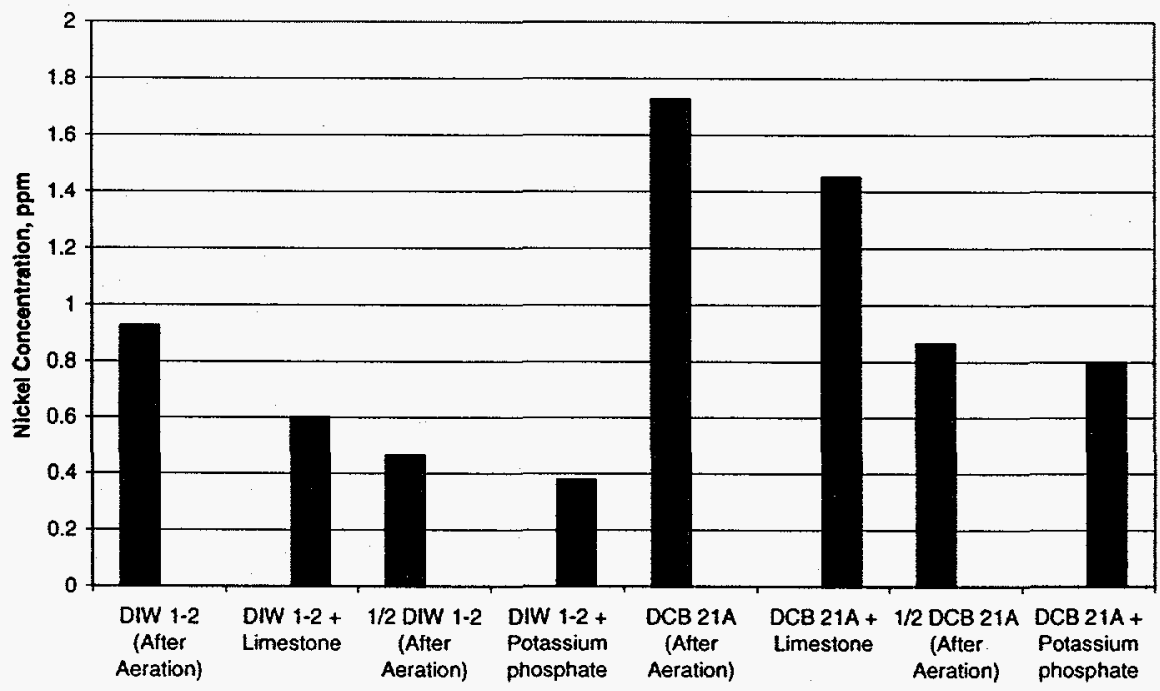


Table 35. Preliminary Laboratory Observations Lab Test \#4

\begin{tabular}{|c|c|c|c|c|c|c|}
\hline & DIW-1-2 & $\begin{array}{l}\text { Aeration \& } \\
20 \% \text { Limestone } \\
\% \text { Change }\end{array}$ & $\begin{array}{l}\text { Aeration \& } \\
\text { Potassium } \\
\text { Phosphate } \\
\text { \%Change }\end{array}$ & DCB-21A & $\begin{array}{l}\text { Aeration \& } \\
20 \% \text { Limestone } \\
\% \text { Change }\end{array}$ & $\begin{array}{l}\text { Aeration \& } \\
\text { Potassium } \\
\text { Phosphate } \\
\text { \%Change }\end{array}$ \\
\hline Aluminum & 82.116 & -99.6 & -99.3 & 300.1 & -99.9 & -8.2 \\
\hline Calcium & 62.962 & 508.7 & -1.6 & 175.9 & 658.2 & 3.5 \\
\hline Chromium & 11.240 & -99.5 & -94.9 & 0.3 & -76.3 & -41.5 \\
\hline Iron & 124.816 & -72.7 & -42.7 & 130.9 & -92.4 & -66.6 \\
\hline Magnesium & 34.080 & 45.7 & 3.9 & 112.0 & 17.7 & 0.2 \\
\hline Manganese & 6.403 & -49.0 & -19.3 & 11.4 & -29.9 & -0.5 \\
\hline Nickel & 0.964 & -37.8 & -21.8 & 1.7 & -14.0 & -5.6 \\
\hline Phosphorus & $<0.273$ & & & 0.3 & & \\
\hline Silica & 77.742 & -40.1 & 5.0 & 120.7 & -43.3 & -0.5 \\
\hline
\end{tabular}

\subsection{System Chemistry}

Based on initial conditions and the solubility diagram shown in Figure 52, a minor $\mathrm{pH}$ adjustment was sufficient to precipitate the chromium phases in our groundwater. Nickel, on the other hand, was more difficult to precipitate by $\mathrm{pH}$ adjustment alone. Figure 53, shows the nickel solubility diagrams in the presence of $9500 \mathrm{mg} / \mathrm{L}$ of phosphate. Less phosphate would only shift the solubility curves farther to the right requiring even higher $\mathrm{pHs}$ to precipitate the nickel phases. For this reason, it is assumed that nickel must co-precipitate with other metals. Nickel would partition strongly into ferrous iron phases as a co-precipitate because of the similarity in charge and cation radii of nickel and ferrous iron. Figure 54 suggests that there is significant dissolved ferrous iron in the system and phases such as ferrous phosphate may precipitate. Nickel may also co-precipitate with ferric iron phases. This may explain why aeration promoted the removal of nickel from the contaminated groundwater. Ferric iron phases are less soluble than ferrous iron phases and more complete iron removal can expected ferrous iron is oxidized. Greater precipitation of iron may, in turn, result in more effective removal of nickel. 
Figre 52 Solubility of Amorhous orfat3

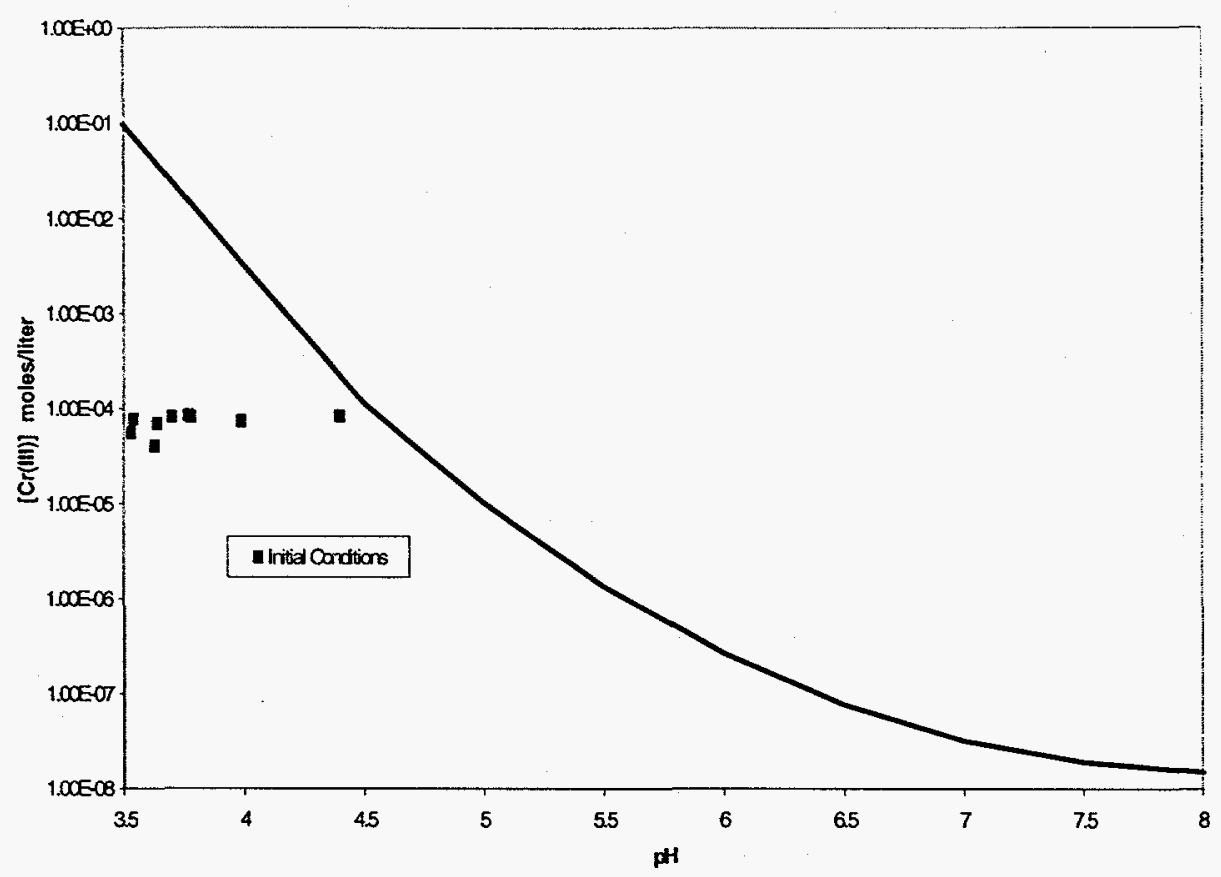

Figure 53 Solubility of Nidel Pheses (Total Phosphate $=0.1$ molesliter)

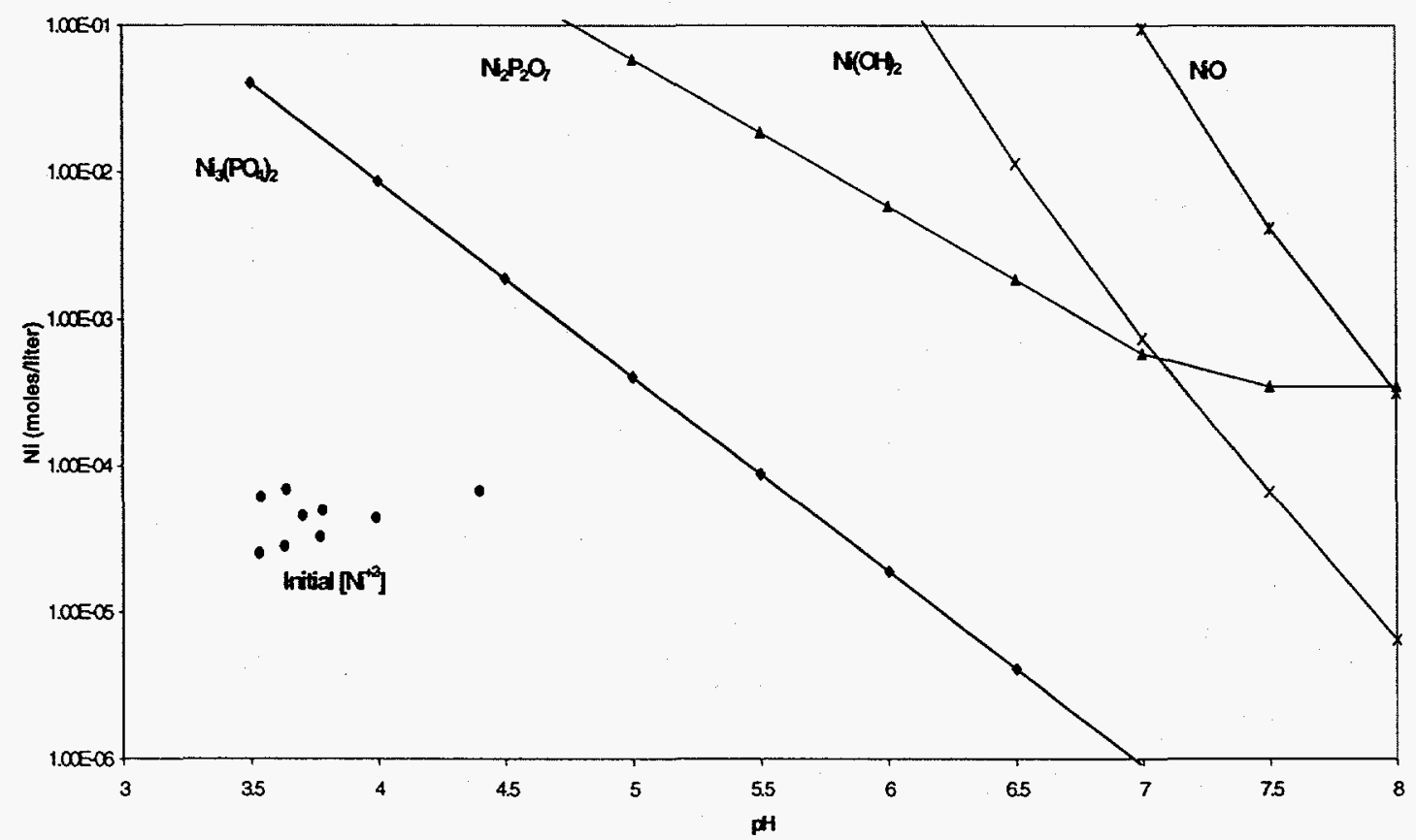


Figre 54. B-pHDagram of Iran in Solution

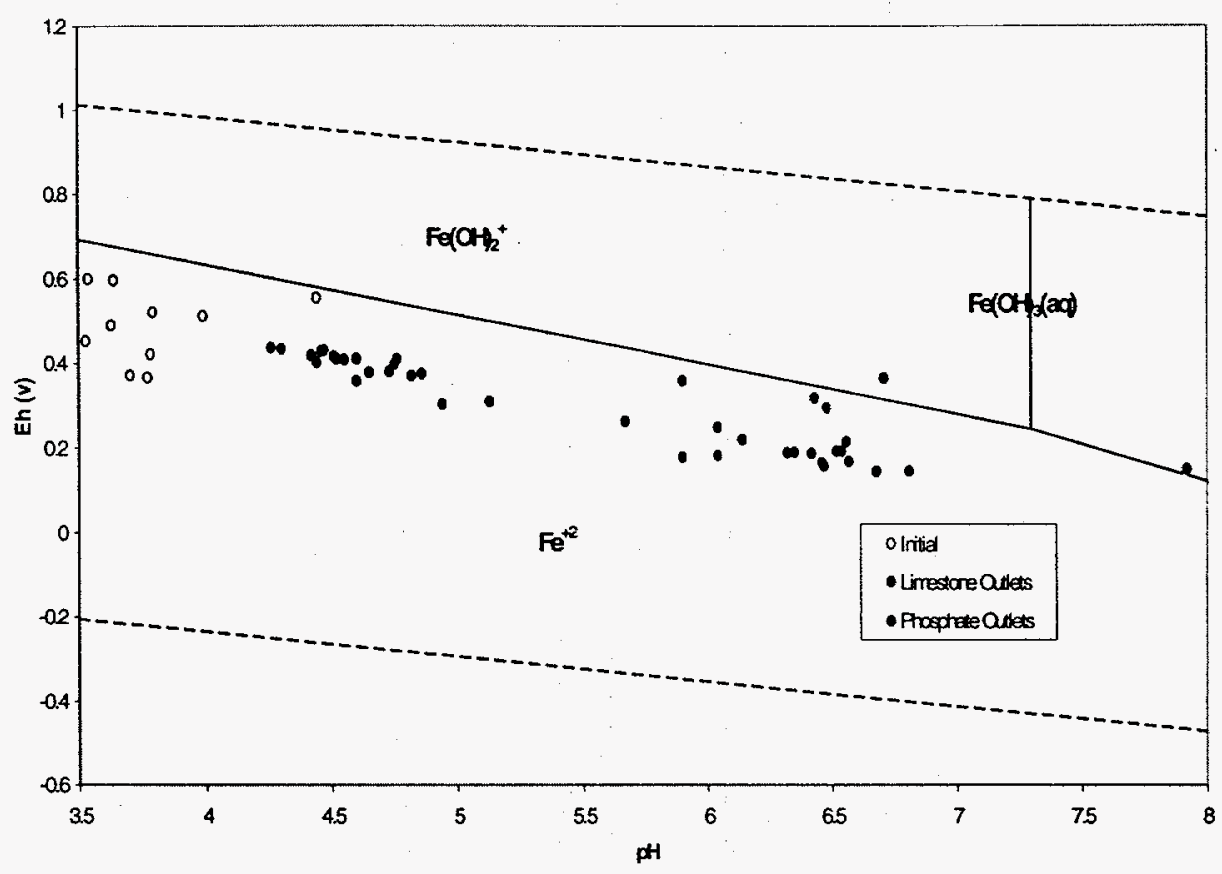

\section{CONCLUSIONS, RECOMMENDATIONS, AND GEOSIPHON TREATMENT SYSTEM CONCEPTUAL DESIGN}

As determined from Field Run \#1, contaminants of concern can be removed by precipitation in the first bulk precipitation canister and by cation exchange or further increases in $\mathrm{pH}$ in the second polishing canister. From the preliminary results of Field Run \#1, it appears as long as the bulk precipitation process is successful, the polishing process works rather well. Significant emphasis was placed on these campaigns to refine the bulk precipitation process. It should be noted that the overall treatment process should consider all potential combinations to provide the best possible treatment.

While these results are preliminary, the following general overall conclusions for the entire operating regime are deduced:

- Both aluminum and chromium can be effectively treated with limestone and phosphate

- Iron can also be treated with limestone and phosphate.

- While iron is significantly removed, treatments are not as effective as those for aluminum and chromium.

- Treatments that enhance iron removal also enhance nickel removal

- Nickel is probably coprecipitated with iron. 
Based on the preliminary data results and field observations, a conceptual design which includes GeoSiphon cells is under evaluation. GeoSiphon Cells are passive groundwater treatment systems which utilize siphon flow between two points of natural head difference to induce greater than natural contaminated groundwater flow through a treatment train without the requirement for external energy input. The treatment train utilized in GeoSiphon Cells can include permeable treatment materials, aeration, chemical addition, etc.

The conceptual GeoSiphon Cell configuration includes extraction of the contaminated water from the mound created by the barrier wall system by siphon to a downgradient treatment system which may consist of an oxidizing step such as aeration followed by a permeable treatment media or treatment media combination. The siphon flow will be induced by the natural hydraulic head difference between the mound upgradient of the wall and the adjacent D-Area discharge stream, which is a lower hydraulic head. A schematic is shown in Figure 55. Further development of this concept is anticipated in FY99.

Advantages of this conceptual design include:

- Utilization of the existing barrier wall system as part of a treatment system for the D-Area CPRB metals contaminated groundwater. The existing barrier wall system represents a significant past Office of Science and Technology (OST) investment.

- Utilize natural hydraulic driving forces to induce flow for remediation.

- Treatment within the most heavily contaminated portion of the D-Area CPRB plume.

- Removable treatment options so that heavy metals can be collected and recycled.

- Potentially accelerate clean up, can be applied to a broader range of site conditions, and can increase treatment media longevity and effectiveness.

- Minimize media armoring and/or cementation, and formation pluggage.

- Directly meeting the needs of the EM-40 program (Environmental Restoration).

Although the data presented in this report are preliminary, the following should be considered for further evaluation:

- All data should be looked at in greater detail by statistical analysis, mass balance, etc.

- Comparison of data and treatment effectiveness should be performed

- In particular, ammonium phosphate should be evaluated more closely due to its performance in Run \#4. 
Figure 55. D-Area GeoSiphon Cell Treatment System - Conceptualization

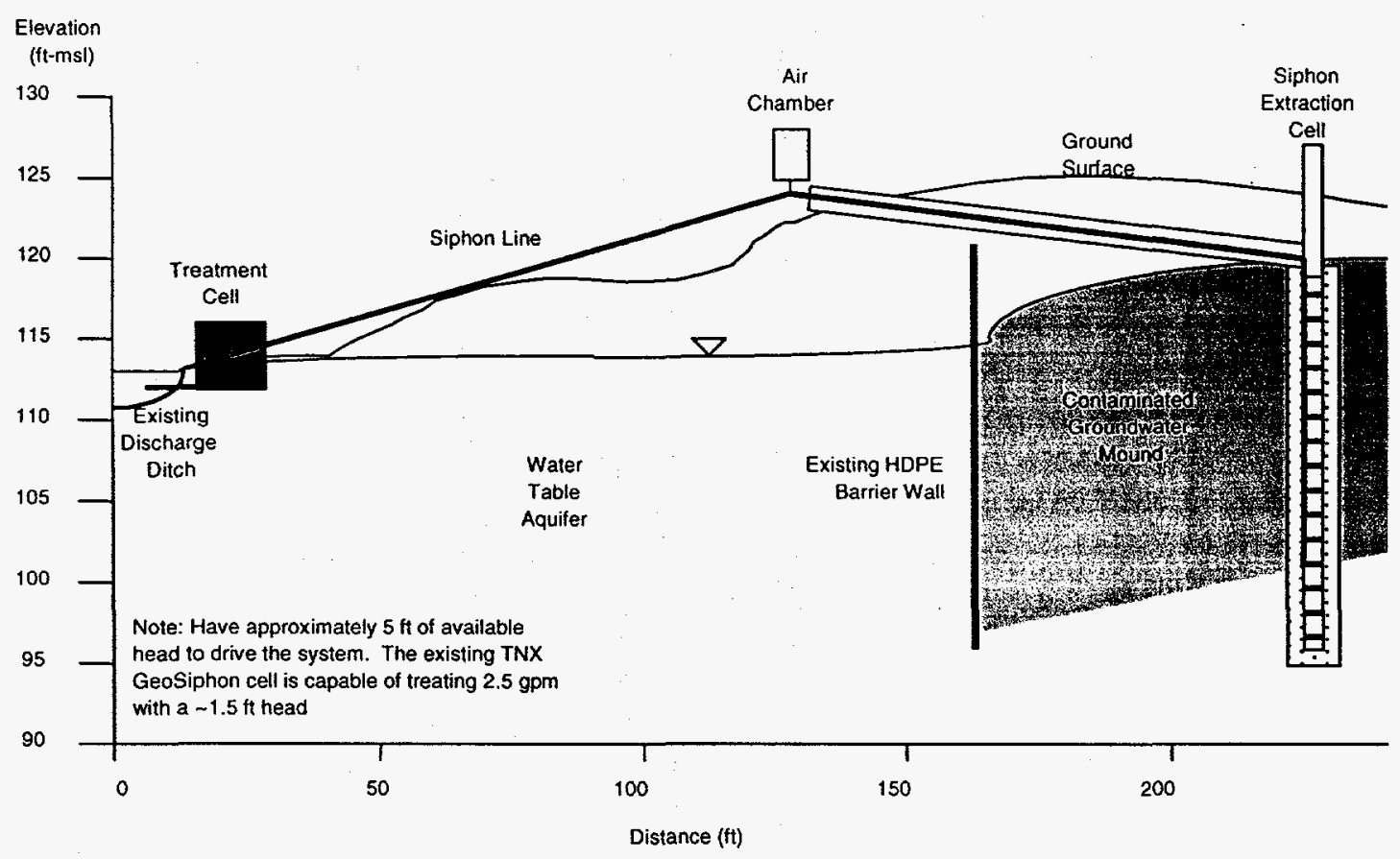

\section{KEY CONTACTS AND PARTICIPANTS}

US Department of Energy

Dave Robertson, Reactive Barrier Product Line Manager

Michelle Ewart, DOE-SR

Jim Wright, Subcontaminants Focus Area

WSRC Environmental Restoration Department

Harry Long, Facility Custodian

Coleman Miles, Technical Lead

WSRC Savannah River Technology Center

Miles Denham, Senior Geochemist

Mark Phifer, Principal Engineer

Frank Sappington, Senior Engineer

Fatina Ann Washburn, Senior Engineer

Chuck Betivas, Technical Assistant

WSRC Environmental Protection Department

Bill Payne, Permitting Coordinator

$\underline{\text { SCE\&G }}$

Mark Ferguson, Environmental Coordinator

Jean Claude Younan, Environmental Programs 


\section{REFERENCES}

1. RW Gillham, DW Blowes, CJ Ptacek, and SF O'Hannesin, 1994. In-situ remediation: Scientific basis for current and future technologies. Thirty-Third Hanford Symposium on Health and Environment.

2. DW Blowes, CJ Ptacek, JG Bain, KR Waybrant, and WD Robertson, 1995.

Treatment of mine drainage water using in-situ permeable reactive walls. Conference on Mining and Environment, Sudbury, Ontario, May 28-June 1.

3. KR Waybrant, DW Blowes, and FJ Ptacek, 1995. Selections of reactive mixtures for the prevention of acid mine drainage using porous reactive walls.

4. DW Blowes, KR Waybrant, JF Bain, and WD Robertson, 1995. In-situ treatment of drainage water using porous reactive walls. Conference on Mining and Environment, Sudbury, Ontario, May 28-June1.

5. DW Blowes, and CJ Ptacek, 1992. Geochemical remediation of groundwater by permeable reactive walls: Removal of chromate by reaction with iron bearing solids. Proceedings of the Surface Restoration Conference, Third International Conference of Groundwater Quality Research, June 21-24, Dallas, Texas, pp. 214-216.

6. RW Gillham, DW Blowes, CJ Ptacek, and SF O'Hannesin, 1994. "Use of zerovalent metals in-situ remediation of contaminated groundwater." 33rd Hanford Symposium of Health and the Environment, Pasco, Washington, Nov. 7-11.

7. Remediation Technologies Development Forum Permeable Barriers Action Team Meeting Summary, Dec. 11-12, 1996, Denver, CO.

8. EC Thornton, Selection of field demonstration site for in situ chemical treatment of soil testing activities, WHC-SD-EN-ES-042, Westinghouse Hanford Company, Richland, Washington, 1994.

9. GM Haggerty, and RS Bowman, "Symposium of Chromate and Other Inorganic Anions by Organo-Zeolite, "Environ. Sci. Technol., Vol. 28, No. 3, 1994.

10. I Anghel, J. Whitworth, S Roy, RS Bowman, "Remediation of subsurface chromium (VI) and PCD contaminated with surface-modified zeolites: Saturated column studies," Proc. WERC/HSRC '97 Joint Conf. on the Environment, 22-24 April 1997, Albuquerque, NM.

11. TM Sivavec, PD Mackenzie, DP Horney, and SS Baghel, "Redox active media for permeable reactive barrier, "International Contaminant Technology Conference, Feb 912, 1997, Petersburg, FL.

12. Phifer, MA, "Preliminary Engineering Report- Proposed TNX GeoSiphon Cell Phase II Demonstration", submitted to the South Carolina Department of Health and Environmental Control, June 10, 1997.

13. Phifer, MA, "Expert Panel Report-MWMF Groundwater Southwest PlumeGeoFlow Cell Remediation Option", submitted to Environmental Restoration Engineering, May 30, 1997.

\section{APPENDICES}

Appendix A: Compiled Data 
APPENDIX A

COMPILED DATA 


\section{FIELD RUN \#1}



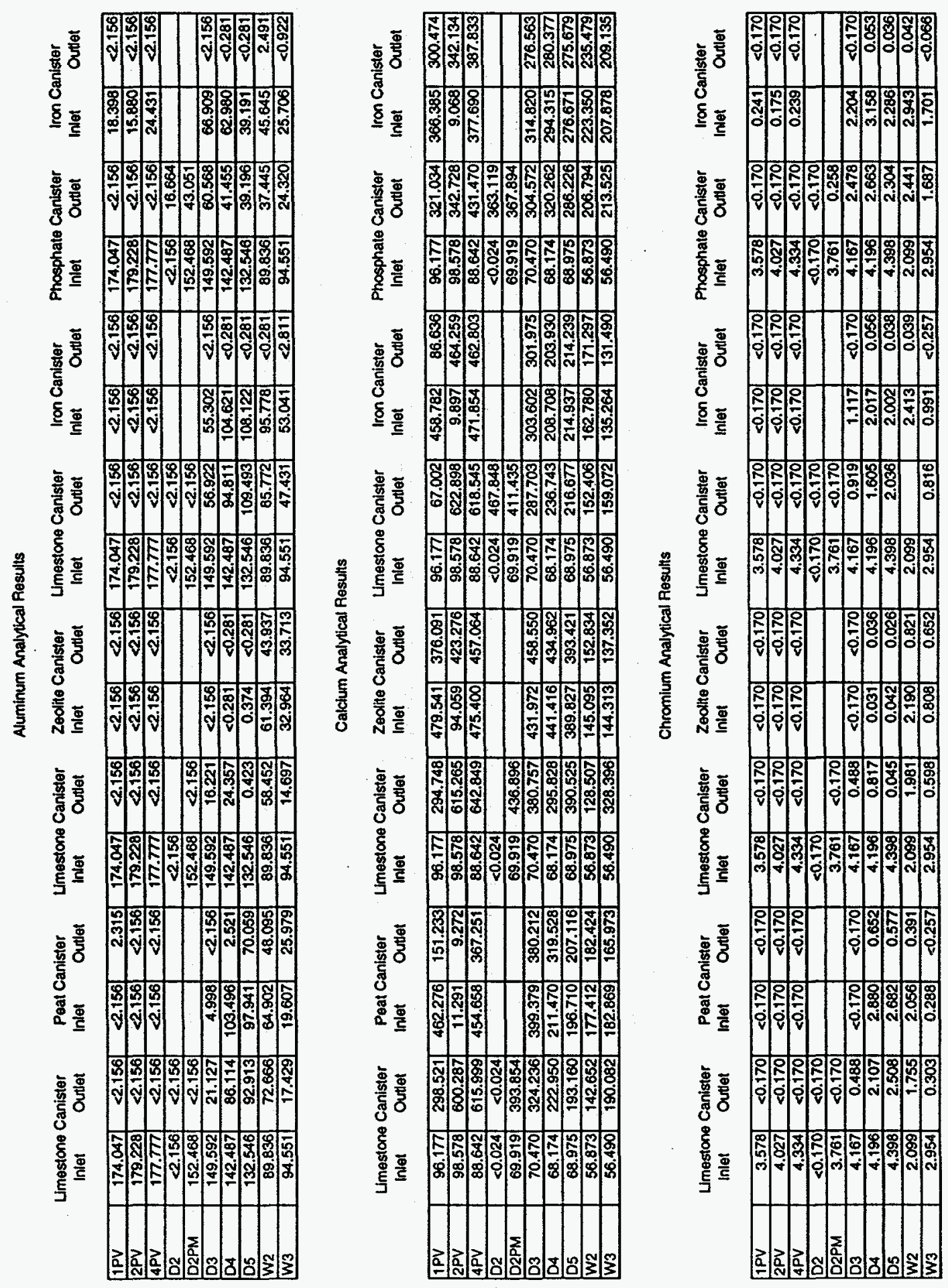

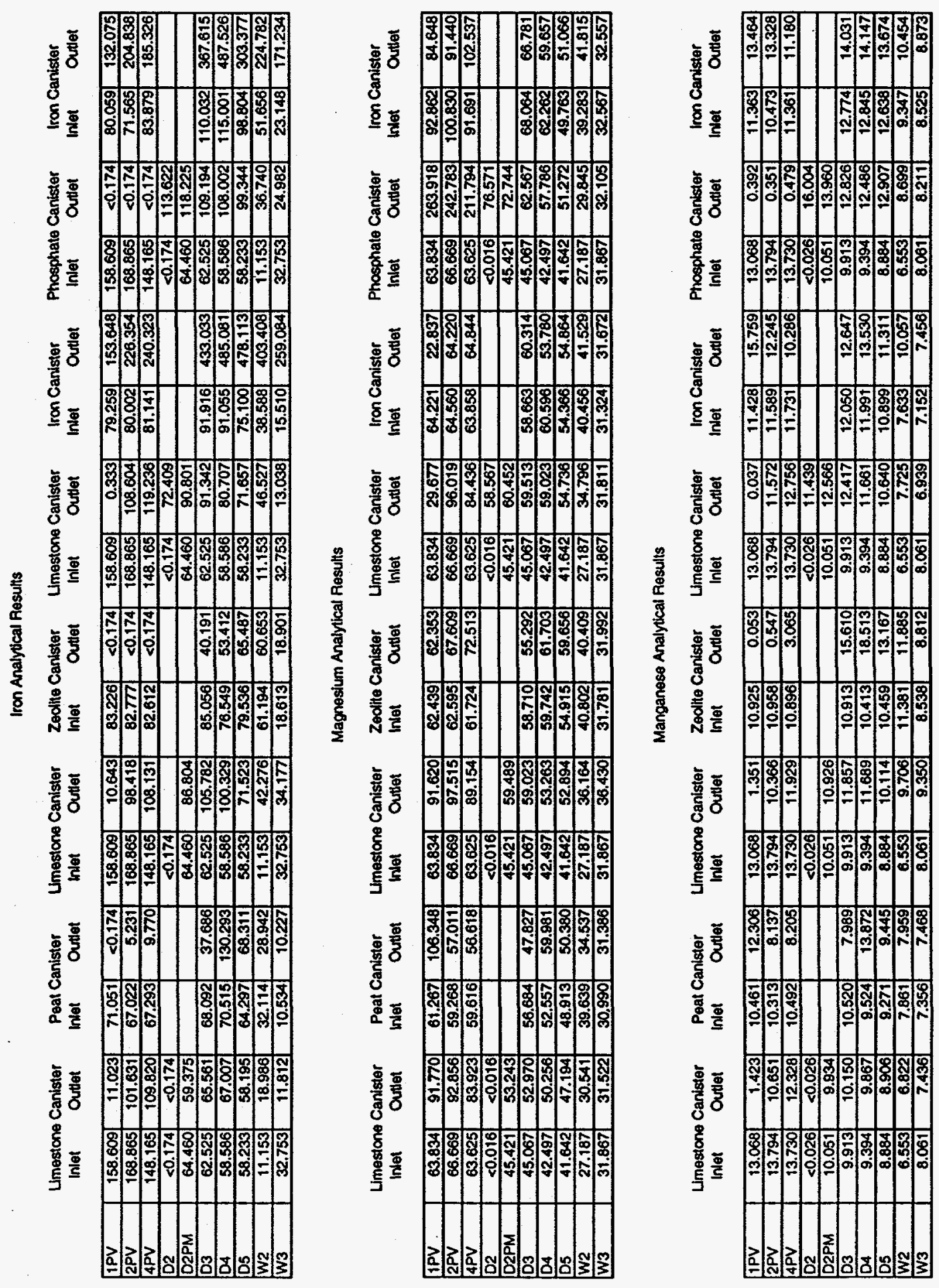

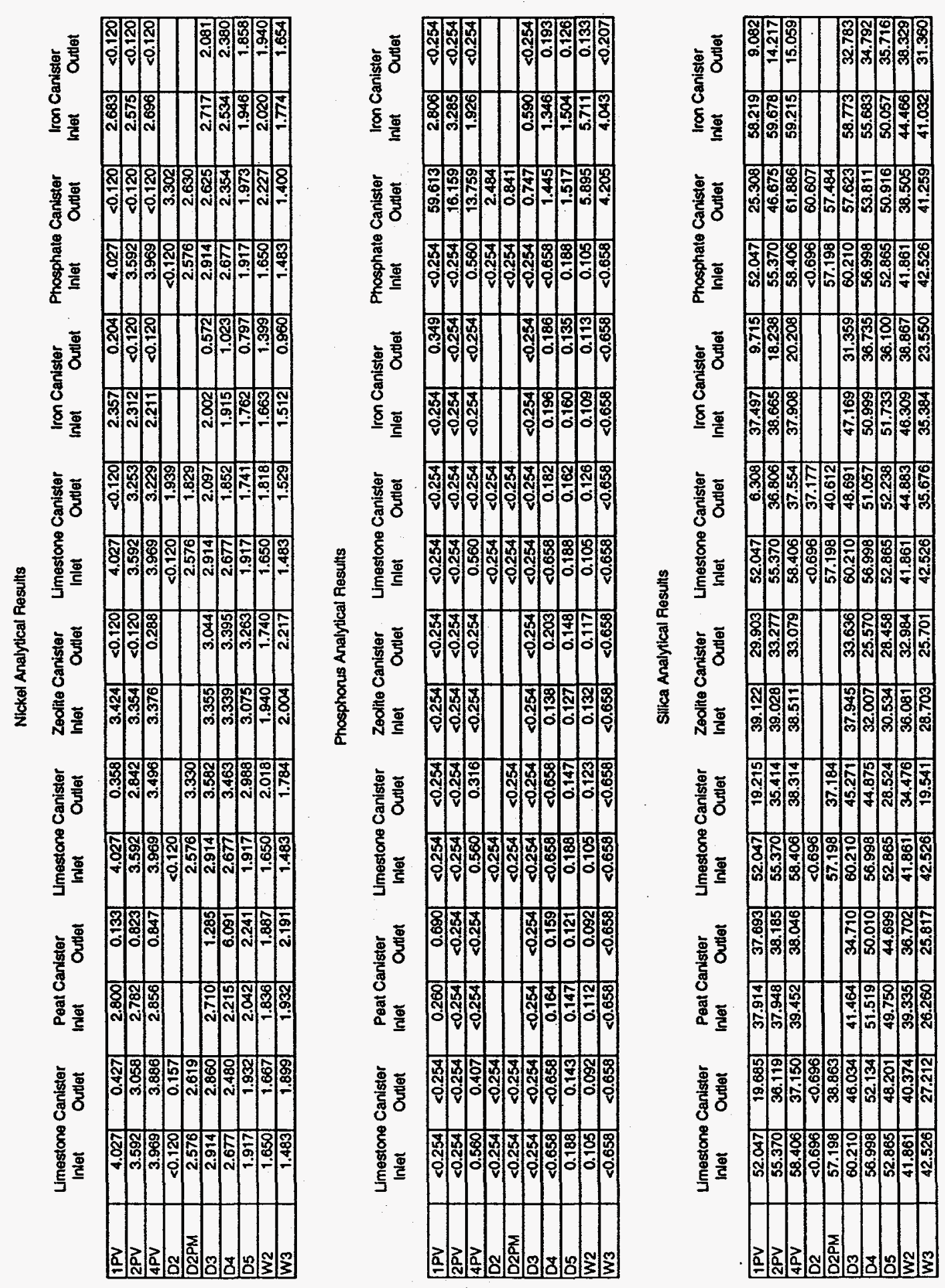


\section{is}

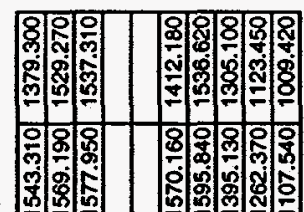

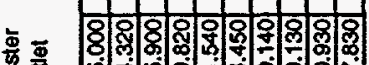

壱

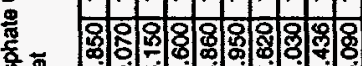

荌昰

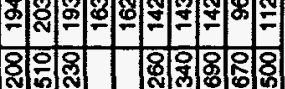

旁

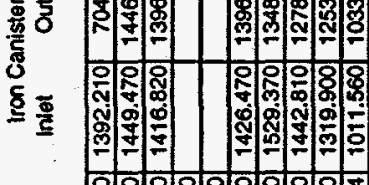

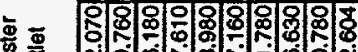

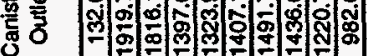

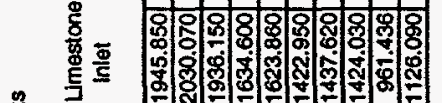

章

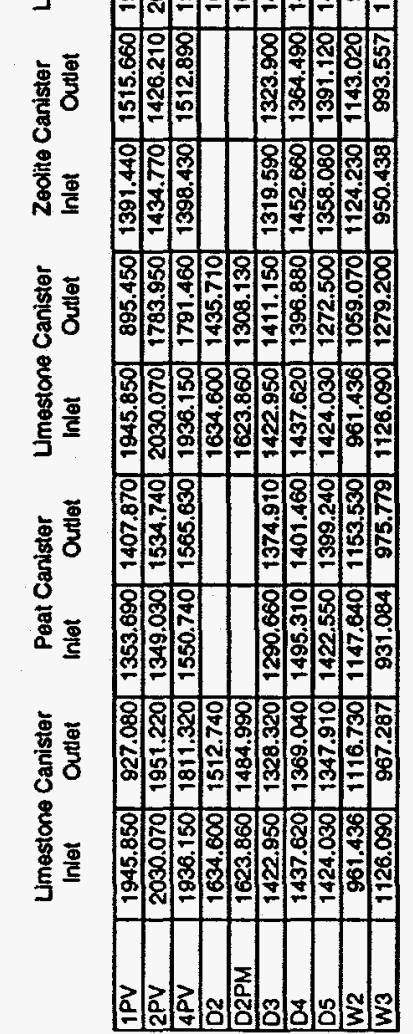

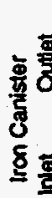

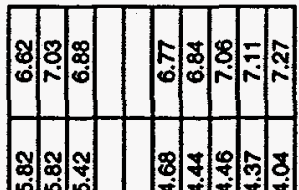

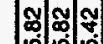

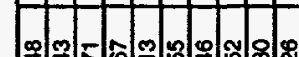

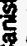

S

案

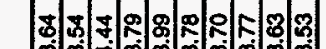

离

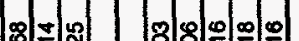

恖

흐 홀

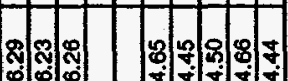

嘉要

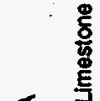

超

弮

†.

壱

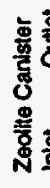

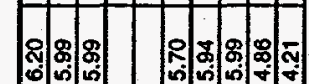

照

尊

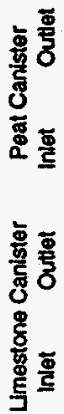
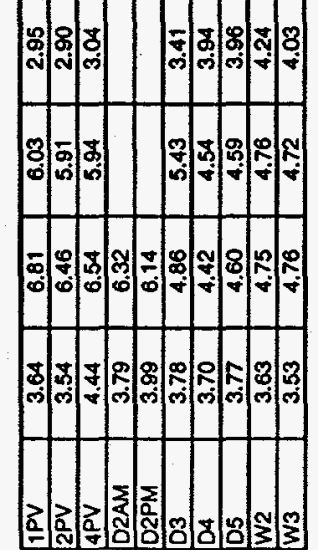

$\div=$

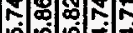
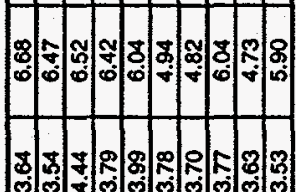

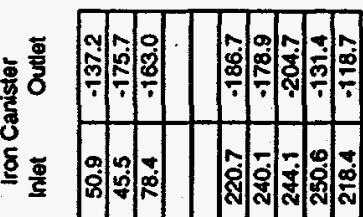

密要

常

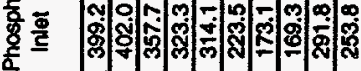

要要

$50-\infty$

密

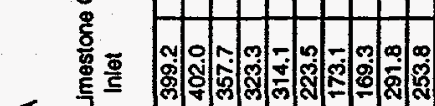

赵

至

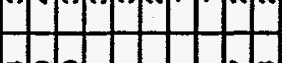

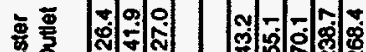
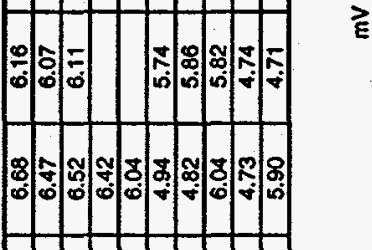

总

옹.

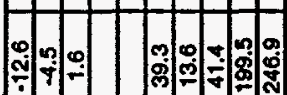

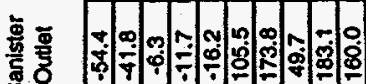

密

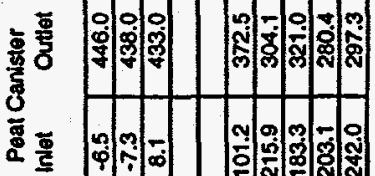

- $\infty-1.0 .0 .0$.

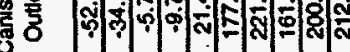

\%

总

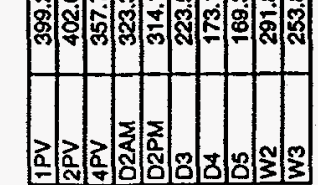




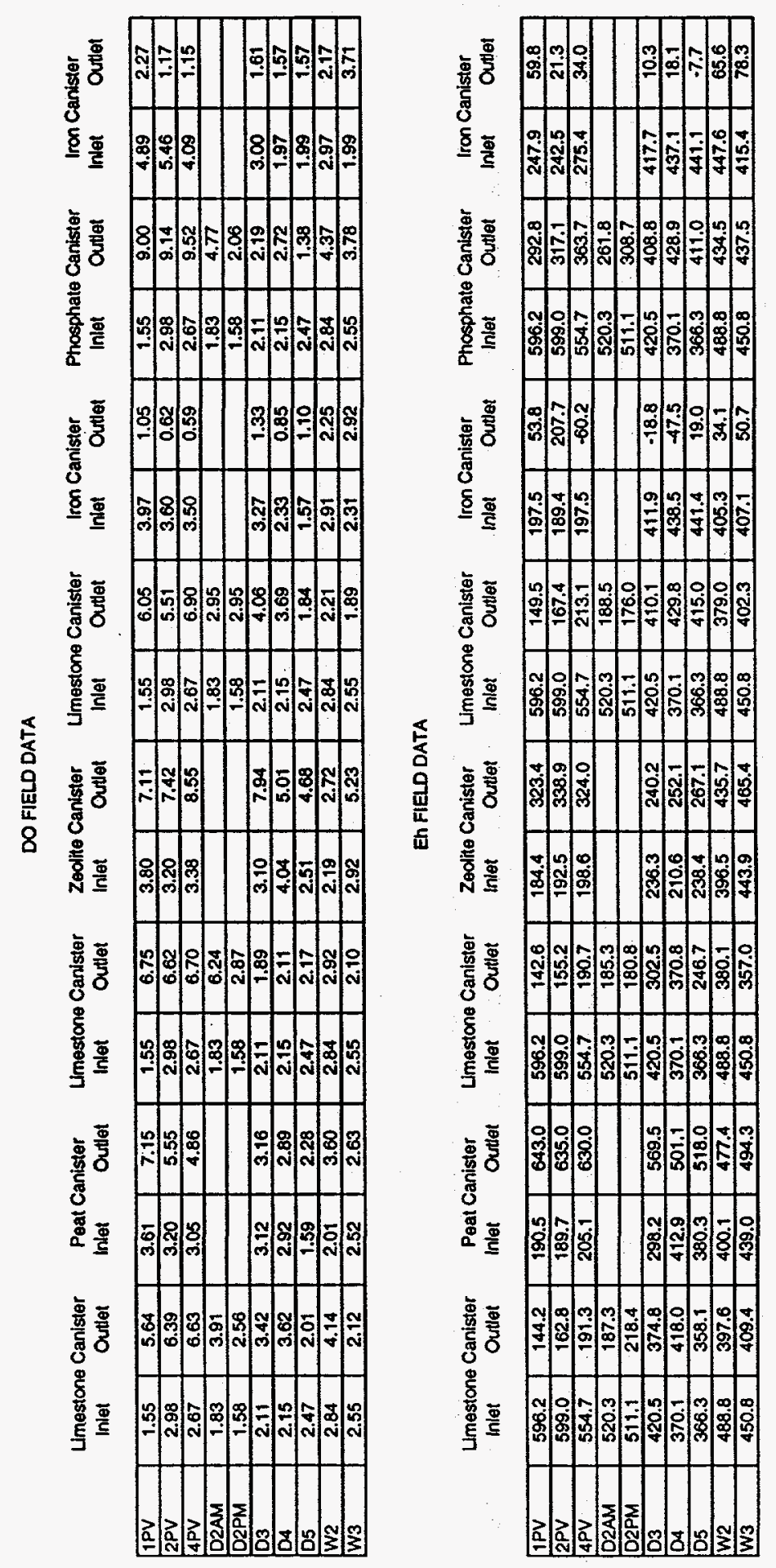


FILED RUN \#2 
Aluminum Analytical Results

\begin{tabular}{llllllll}
\multicolumn{4}{c}{ Limestone } & & \multicolumn{2}{c}{ Phosphate Rock } \\
Raw & Media & Raw $1 / 2$ Raw & Treated & Raw & Media & Raw & $1 / 2$ Raw \\
Water & Solution & Groundwater Groundwater Groundwater Water & Solution & Groundwater Groundwater Groundwater
\end{tabular}

\begin{tabular}{|c|c|c|c|c|c|c|c|c|c|c|}
\hline Day 1 & $<0.281$ & $<0.281$ & 72.188 & 36.094 & 22.590 & $<0.281$ & 0.417 & 72.188 & 36.094 & 22.283 \\
\hline Day 2 & $<0.281$ & $<0.281$ & 70.122 & 35.061 & 25.670 & $<0.281$ & $<2.811$ & 70.122 & 35.061 & 34.357 \\
\hline Day 3 & $<0.281$ & $<0.281$ & 78.877 & 39.439 & 35.943 & $<0.281$ & $<0.281$ & 78.877 & 39.439 & $29: 336$ \\
\hline Day 4 & $<0.260$ & $<0.260$ & 87.015 & 43.508 & 37.925 & $<0.260$ & 50.260 & 87.015 & 43.508 & 26.258 \\
\hline Week 2 & $<0.116$ & $<0.116$ & 76.204 & 38.102 & 33.354 & $<0.116$ & $<0.116$ & 76.204 & 38.102 & 45.954 \\
\hline Week 3 & $<0.260$ & $<0.260$ & 87.281 & 43.641 & 30.116 & $<0.260$ & $<0.260$ & 87.281 & 43.641 & 8.421 \\
\hline
\end{tabular}

Calcium Analytical Results

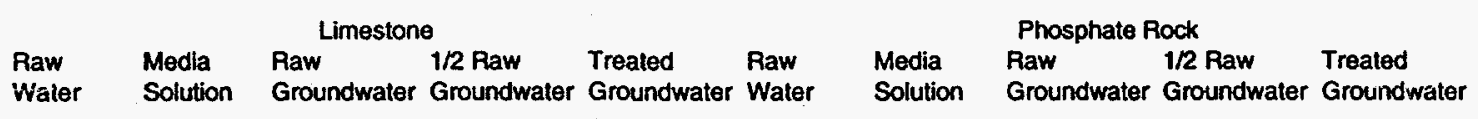

\begin{tabular}{|c|c|c|c|c|c|c|c|c|c|c|}
\hline Day 1 & 0.536 & 9.622 & 56.750 & 28.375 & 45.035 & 0.536 & 375.851 & 56.750 & 28.375 & 230.205 \\
\hline Day 2 & 0.505 & 4.171 & 49.792 & 24.896 & 34.160 & 0.505 & 53.075 & 49.792 & 24.896 & 25.982 \\
\hline Day 3 & 1.736 & 3.831 & 49.356 & 24.678 & 28.031 & 1.736 & 12.132 & 49.356 & 24.678 & 31.735 \\
\hline Day 4 & 0.771 & 3.977 & 49.166 & 24.583 & 26.032 & 0.771 & 9.403 & 49.166 & 24.583 & 25.960 \\
\hline Week 2 & 0.544 & 3.605 & 47.397 & 23.699 & 21.319 & 0.544 & 5.417 & 47.397 & 23.699 & 34.851 \\
\hline Week 3 & 0.613 & 3.817 & 47.742 & 23.871 & 18.828 & 0.613 & 3.538 & 47.742 & 23.871 & 15.011 \\
\hline
\end{tabular}

Chromium Analytical Results

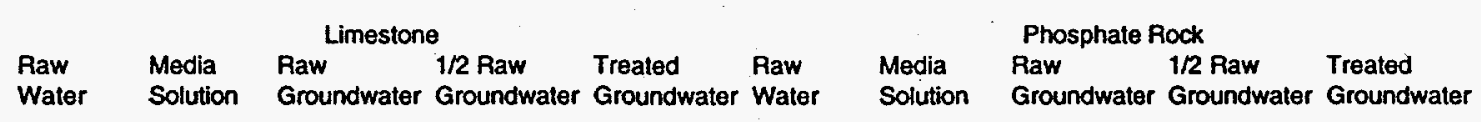

\begin{tabular}{|c|c|c|c|c|c|c|c|c|c|c|}
\hline Day 1 & $<0.023$ & $<0.023$ & 5.570 & 2.785 & 1.376 & $<0.023$ & 0.048 & 5.570 & 2.785 & 2.474 \\
\hline Day 2 & $<0.023$ & $<0.023$ & 3.967 & 1.984 & 1.474 & $<0.023$ & $<0.233$ & 3.967 & 1.984 & 1.588 \\
\hline Day 3 & $<0.023$ & $<0.023$ & 4.502 & 2.251 & 1.776 & $<0.023$ & $<0.023$ & 4.502 & 2.251 & 1.470 \\
\hline Day 4 & $<0.017$ & $<0.017$ & 4.799 & 2.400 & 1.814 & $<0.017$ & $<0.017$ & 4.799 & 2.400 & 1.217 \\
\hline Week 2 & $<0.009$ & $<0.009$ & 4.246 & 2.123 & 1.624 & $<0.009$ & $<0.009$ & 4.246 & 2.123 & 2.241 \\
\hline Week 3 & $<0.017$ & $<0.017$ & 4.943 & 2.472 & 1.437 & $<0.017$ & $<0.017$ & 4.943 & 2.472 & 0.230 \\
\hline
\end{tabular}

Iron Analytical Results

Raw Media Raw Limestone Treated Raw Media Raw Phosphate Rock

Water Solution Groundwater Groundwater Groundwater Water Solution Groundwater Groundwater Groundwater

\begin{tabular}{|c|c|c|c|c|c|c|c|c|c|c|}
\hline Day 1 & $<0.020$ & $<0.020$ & 63.423 & 31.712 & 31.487 & $<0.020$ & 0.036 & 63.423 & 31.712 & 31.114 \\
\hline Day 2 & $<0.020$ & $<0.020$ & 39.808 & 19.904 & 16.938 & $<0.020$ & $<0.199$ & 39.808 & 19.904 & 17.052 \\
\hline Day 3 & 0.311 & $<0.020$ & 32.549 & 16.275 & 14.654 & 0.311 & $<0.020$ & 32.549 & 16.275 & 14.053 \\
\hline Day 4 & $<0.020$ & $<0.020$ & 25.612 & 12.806 & 12.137 & $<0.02$ & $<0.020$ & 25.612 & 12.806 & 12.193 \\
\hline Week 2 & 1.337 & $<0.009$ & 29.360 & 14.680 & 10.659 & 1.337 & $<0.009$ & 29.360 & 14.680 & 19.994 \\
\hline Week 3 & 0.146 & $<0.020$ & 29.627 & 14.814 & 10.731 & 0.146 & $<0.020$ & 29.627 & 14.814 & 5.422 \\
\hline
\end{tabular}


Magnesium Analytical Results

\begin{tabular}{|c|c|c|c|c|c|c|c|c|c|c|}
\hline & \multicolumn{5}{|c|}{ Limestone } & \multicolumn{5}{|c|}{ Phosphate Rock } \\
\hline & $\begin{array}{l}\text { Raw } \\
\text { Water }\end{array}$ & $\begin{array}{l}\text { Media } \\
\text { Solution }\end{array}$ & $\begin{array}{l}\text { Raw } \\
\text { Groundwater }\end{array}$ & $\begin{array}{l}\text { 1/2 Raw } \\
\text { Groundwater }\end{array}$ & $\begin{array}{l}\text { Treated } \\
\text { Groundwater }\end{array}$ & $\begin{array}{l}\text { Raw } \\
\text { Water }\end{array}$ & $\begin{array}{l}\text { Media } \\
\text { Solution }\end{array}$ & $\begin{array}{l}\text { Raw } \\
\text { Groundwater }\end{array}$ & $\begin{array}{l}\text { 1/2 Raw } \\
\text { Groundwater }\end{array}$ & $\begin{array}{l}\text { Treated } \\
\text { Groundwater }\end{array}$ \\
\hline Day 1 & 0.192 & 4.514 & 31.713 & 15.857 & 16.694 & 0.192 & 180.995 & 31.713 & 15.857 & 110.634 \\
\hline Day 2 & 0.192 & 1.325 & 25.383 & 12.692 & 13.061 & 0.192 & 19.349 & 25.383 & 12.692 & 14.166 \\
\hline Day 3 & 1.020 & 1.115 & 25.555 & 12.778 & 13.877 & 1.020 & 2.131 & 25.555 & 12.778 & 12.756 \\
\hline Day 4 & 0.199 & 0.931 & 25.361 & 12.681 & 12.786 & 0.199 & 1.289 & 25.361 & 12.681 & 10.801 \\
\hline Week 2 & 0.227 & 0.685 & 24.682 & 12.341 & 11.614 & 0.227 & 0.935 & 24.682 & 12.341 & 17.025 \\
\hline Week 3 & 0.201 & 0.482 & 26.045 & 13.023 & 9.987 & 0.201 & 0.565 & 26.045 & 13.023 & 7.155 \\
\hline
\end{tabular}

Manganese Analytical Results

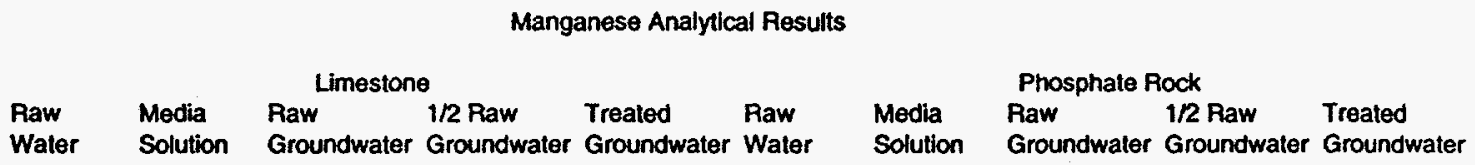

\begin{tabular}{|l|r|r|r|r|r|r|r|r|r|r|}
\hline Day 1 & $<0.006$ & 0.013 & 7.388 & 3.694 & 3.587 & $<0.006$ & 0.526 & 7.388 & 3.694 & 3.817 \\
\hline Day 2 & $<0.006$ & $<0.006$ & 6.023 & 3.012 & 2.592 & $<0.006$ & $<0.061$ & 6.023 & 3.012 & 2.933 \\
\hline Day 3 & 0.188 & $<0.006$ & 5.869 & 2.935 & 2.955 & 0.188 & 0.012 & 5.869 & 2.935 & 2.577 \\
\hline Day 4 & $<0.006$ & 0.006 & 5.636 & 2.818 & 2.699 & $<0.006$ & $<0.006$ & 5.636 & 2.818 & 2.301 \\
\hline Weak 2 & 0.010 & $<0.003$ & 5.618 & 2.809 & 2.387 & 0.010 & 0.003 & 5.618 & 2.809 & 3.797 \\
\hline Weak 3 & $<0.006$ & $<0.006$ & 5.770 & 2.885 & 2.100 & $<0.006$ & $<0.006$ & 5.770 & 2.885 & 1.485 \\
\hline
\end{tabular}

Nickel Analytical Results

\begin{tabular}{|c|c|c|c|c|c|c|c|c|c|}
\hline \multirow[b]{2}{*}{$\begin{array}{l}\text { Raw } \\
\text { Water }\end{array}$} & \multicolumn{3}{|c|}{ Limestone } & \multirow[b]{2}{*}{$\begin{array}{l}\text { Treated } \\
\text { Groundwater }\end{array}$} & \multirow[b]{2}{*}{$\begin{array}{l}\text { Raw } \\
\text { Water }\end{array}$} & \multicolumn{3}{|c|}{ Phosphate Rock } & \multirow[b]{2}{*}{$\begin{array}{l}\text { Treated } \\
\text { Groundwater }\end{array}$} \\
\hline & $\begin{array}{l}\text { Media } \\
\text { Solution }\end{array}$ & $\begin{array}{l}\text { Raw } \\
\text { Groundwater }\end{array}$ & $\begin{array}{l}\text { 1/2 Raw } \\
\text { Groundwater }\end{array}$ & & & $\begin{array}{l}\text { Media } \\
\text { Solution }\end{array}$ & $\begin{array}{l}\text { Raw } \\
\text { Groundwater }\end{array}$ & $\begin{array}{l}\text { 1/2 Raw } \\
\text { Groundwater }\end{array}$ & \\
\hline$<0.037$ & $<0.037$ & 0.967 & 0.4835 & 0.452 & $<0.037$ & 0.081 & 0.967 & 0.484 & $<0.370$ \\
\hline$<0.037$ & $<0.037$ & 0.958 & 0.479 & 0.399 & $<0.037$ & $<0.370$ & 0.958 & 0.479 & $<0.370$ \\
\hline$<0.037$ & $\leq 0.037$ & 1.167 & 0.5835 & 0.538 & $<0.037$ & $<0.037$ & 1.167 & 0.584 & 0.506 \\
\hline$<0.029$ & $<0.029$ & 1.077 & 0.5385 & 0.519 & $<0.029$ & $<0.029$ & 1.077 & 0.539 & 0.465 \\
\hline$<0.015$ & $<0.015$ & 1.246 & 0.623 & 0.488 & $<0.015$ & $<0.015$ & 1.246 & 0.623 & 0.833 \\
\hline$<0.029$ & $<0.029$ & 1.293 & 0.6465 & 0.458 & $<0.029$ & $<0.029$ & 1.293 & 0.647 & 0.323 \\
\hline
\end{tabular}

Phosphorus Analytical Results

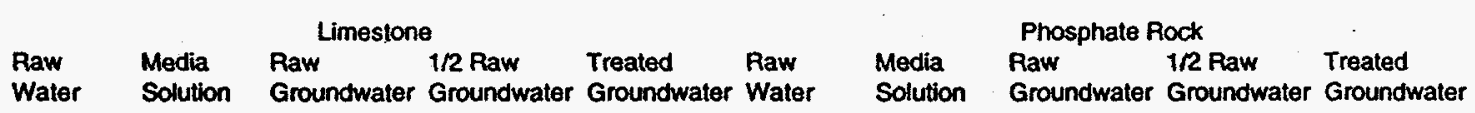

\begin{tabular}{|c|c|c|c|c|c|c|c|c|c|c|}
\hline Day 1 & 0.165 & $<0.066$ & 0.067 & 0.034 & $<0.066$ & 0.165 & 72.447 & 0.067 & 0.034 & 30.954 \\
\hline Day 2 & 0.171 & 0.206 & 0.066 & 0.033 & 1.540 & 0.171 & 14.572 & 0.066 & 0.033 & $<0.658$ \\
\hline Day 3 & $<0.066$ & 0.174 & 0.070 & 0.035 & $\leq 0.066$ & $<0.066$ & 3.442 & 0.070 & 0.035 & 0.903 \\
\hline Day 4 & 0.171 & 0.141 & 0.050 & 0.025 & 0.061 & 0.171 & 2.209 & 0.050 & 0.025 & 0.596 \\
\hline Week 2 & 0.171 & 0.165 & 0.040 & 0.020 & 0.050 & 0.171 & 1.129 & 0.040 & 0.020 & 0.290 \\
\hline Week 3 & 0.126 & 0.126 & 0.042 & 0.021 & 0.035 & 0.126 & 0.797 & 0.042 & 0.021 & 0.028 \\
\hline
\end{tabular}


Silica Analytical Results

\begin{tabular}{|c|c|c|c|c|c|c|c|}
\hline \multicolumn{3}{|r|}{ Limestone } & \multicolumn{5}{|c|}{ Phosphate Rock } \\
\hline $\begin{array}{l}\text { Raw } \\
\text { Water }\end{array}$ & $\begin{array}{l}\text { Media } \\
\text { Solution }\end{array}$ & $\begin{array}{ll}\text { Raw } & 1 / 2 \text { Raw } \\
\text { Groundwater } & \text { Groundwater }\end{array}$ & $\begin{array}{l}\text { Treated } \\
\text { Groundwater }\end{array}$ & $\begin{array}{l}\text { Raw } \\
\text { Water }\end{array}$ & $\begin{array}{l}\text { Media } \\
\text { Solution }\end{array}$ & $\begin{array}{ll}\text { Raw } & 1 / 2 \text { Raw } \\
\text { Groundwater Groundwater }\end{array}$ & $\begin{array}{l}\text { Treated } \\
\text { Groundwater }\end{array}$ \\
\hline
\end{tabular}

\begin{tabular}{|c|c|c|c|c|c|c|c|c|c|c|}
\hline Day 1 & 3.739 & 4.310 & 36.538 & 18.269 & 19.392 & 3.739 & 55.771 & 36.538 & 18.269 & 47.673 \\
\hline Day 2 & 3.809 & 4.038 & 35.727 & 17.864 & 17.815 & 3.809 & 8.922 & 35.727 & 17.864 & 19.163 \\
\hline Day 3 & 4.268 & 4.003 & 37.789 & 18.895 & 22.105 & 4.268 & 4.096 & 37.789 & 18.895 & 19.006 \\
\hline Day 4 & 3.966 & 3.853 & 38.989 & 19.495 & 20.858 & 3.966 & 3.915 & 38.989 & 19.495 & 17.500 \\
\hline Woek 2 & 3.819 & 3.818 & 38.630 & 19.315 & 19.123 & 3.819 & 3.804 & 38.630 & 19.315 & 27.160 \\
\hline Week 3 & 3.713 & 3.819 & 40.918 & 20.459 & 17.711 & 3.713 & 3.553 & 40.918 & 20.459 & 12.159 \\
\hline
\end{tabular}

Sulfate Anatytical Results

\begin{tabular}{lllllll} 
Raw & \multicolumn{3}{c}{ Limestone } & \multicolumn{3}{c}{ Phosphate Rock } \\
Water & Solution & Raw & Groundwater Groundwater Groundwater Water & Solution & Groundwater Groundwater Groundwater
\end{tabular}

\begin{tabular}{|c|c|c|c|c|c|c|c|c|c|c|}
\hline Day 1 & 2.878 & 9.247 & 850.367 & 425.184 & 526.380 & 2.878 & 1401.030 & 850.367 & 425.184 & 1105.180 \\
\hline Day 2 & 4.136 & 3.254 & 790.969 & 395.485 & 483.964 & 4.136 & 207.156 & 790.969 & 395.485 & 362.720 \\
\hline Day 3 & 25.626 & 2.642 & 812.906 & 406.453 & 500.964 & 25.626 & 69.285 & 812.906 & 406.453 & 363.801 \\
\hline Day 4 & 3.096 & 9.051 & 946.568 & 473.284 & 542.368 & 3.096 & 15.460 & 946.568 & 473.284 & 447.822 \\
\hline Week 2 & 11.459 & 2.909 & 852.186 & 426.093 & 535.305 & 11.459 & 11.495 & 852.186 & 426.093 & 613.236 \\
\hline Week 3 & 4.327 & 2.607 & 958.070 & 479.035 & 365.949 & 4.327 & 11.300 & 958.070 & 479.035 & 271.197 \\
\hline
\end{tabular}

pH Field Results

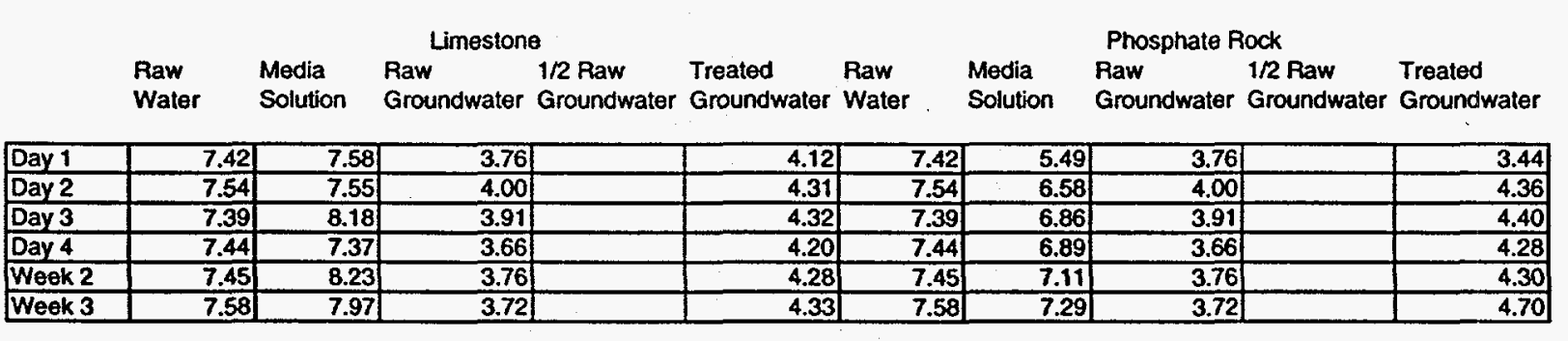

mV Field Results

\begin{tabular}{llllllll} 
Raw & \multicolumn{3}{c}{ Limestone } & & Phosphate Rock \\
Water & Solution & Raw Groundwater Groundwater Groundwater Water & Solution & Groundwater Groundwater Groundwate
\end{tabular}

\begin{tabular}{|c|c|c|c|c|c|c|c|c|}
\hline Day 1 & 83.5 & 30.3 & 97.7 & 90.9 & 83.5 & 101 & 97.7 & 153.1 \\
\hline Day 2 & 138.5 & -51.4 & 88.4 & 73.5 & 138.5 & -11.8 & 88.4 & 80.4 \\
\hline Day 3 & 237.4 & -32.5 & 91.2 & 78.7 & 237.4 & -0.7 & 91.2 & 64.9 \\
\hline Day 4 & 248.3 & 189 & 89.2 & 66.8 & 248.3 & -28.8 & 89.2 & 62.7 \\
\hline Week 2 & 155.4 & 103.7 & 113.3 & 91.4 & 155.4 & -16.6 & 113.3 & 90.7 \\
\hline Week 3 & 376.5 & 380.7 & 88.2 & 118.5 & 376.5 & -35.4 & 88.2 & 126.3 \\
\hline
\end{tabular}

Eh Field Results $(\mathrm{mV}+197 \mathrm{mV})$

\begin{tabular}{lllllll} 
Raw & Media & Raw & Pimestone & & \multicolumn{2}{c}{ Phosphate Rock } \\
Water & Solution & Groundwater Groundwater Groundwater Water & Solution & Raw Groundwater Groundwaler Groundwater
\end{tabular}

\begin{tabular}{|c|c|c|c|c|c|c|c|c|}
\hline Day 1 & 280.5 & 227.3 & 294.7 & 287.9 & 280.5 & 298 & 294.7 & 350.1 \\
\hline Day 2 & 335.5 & 145.6 & 285.4 & 270.5 & 335.5 & 185.2 & 285.4 & 277.4 \\
\hline Day 3 & 434.4 & 164.5 & 288.2 & 275.7 & 434.4 & 196.3 & 288.2 & 261.9 \\
\hline Day 4 & 445.3 & 386 & 286.2 & 263.8 & 445.3 & 168.2 & 286.2 & 259.7 \\
\hline Week 2 & 352.4 & 300.7 & 310.3 & 288.4 & 352.4 & 180.4 & 310.3 & 287.7 \\
\hline Week 3 & 573.5 & 577.7 & 285.2 & 315.5 & 573.5 & 161.6 & 285.2 & 323.3 \\
\hline
\end{tabular}




\section{FIELD RUN \#3}


Aluminum Analytical Results

\begin{tabular}{llllllll}
\multicolumn{2}{c}{ Limestone } & \multicolumn{5}{c}{ Limestone + Phosphate Rock } \\
Raw & Media & Treated & 1/2 Raw Raw & Media Treated & 1/2 Raw \\
GroundWater Solution & Water & Groundwater GroundWater Solution Water & Groundwater DIW 1-2
\end{tabular}

\begin{tabular}{|c|c|c|c|c|c|c|c|c|c|c|}
\hline Day 1 & 145.243 & $<0.030$ & $<0.030$ & 72.622 & 145.243 & $<0.030$ & $<0.301$ & 72.622 & 158.359 & \\
\hline Day 2 & 157.843 & $<0.301$ & $<0.301$ & 78.922 & 157.843 & $<0.301$ & $<0.301$ & 78.922 & & \\
\hline Day 3 & $<0.301$ & $<0.301$ & $<0.301$ & $<0.301$ & $<0.301$ & $<0.301$ & $<0.301$ & $<0.301$ & & \\
\hline Week 2 & 109.530 & 50.301 & 50.301 & 54.765 & 109.530 & $<0.301$ & $<0.301$ & 54.765 & & \\
\hline Week 3 & 114.733 & $<0.301$ & $<0.301$ & 57.367 & 114.733 & $<0.301$ & $<0.301$ & 57.367 & 96.145 & $<0.301$ \\
\hline Week 4 & 114.959 & $<0.301$ & $<0.301$ & 57.480 & 114.959 & $<0.301$ & $<0.301$ & 57.480 & & \\
\hline Week 5 & 107.749 & $<0.030$ & $<0.030$ & 53.875 & 107.749 & $<0.030$ & $<0.030$ & 53.875 & 108.545 & 0.045 \\
\hline
\end{tabular}

Calcium Analytical Results

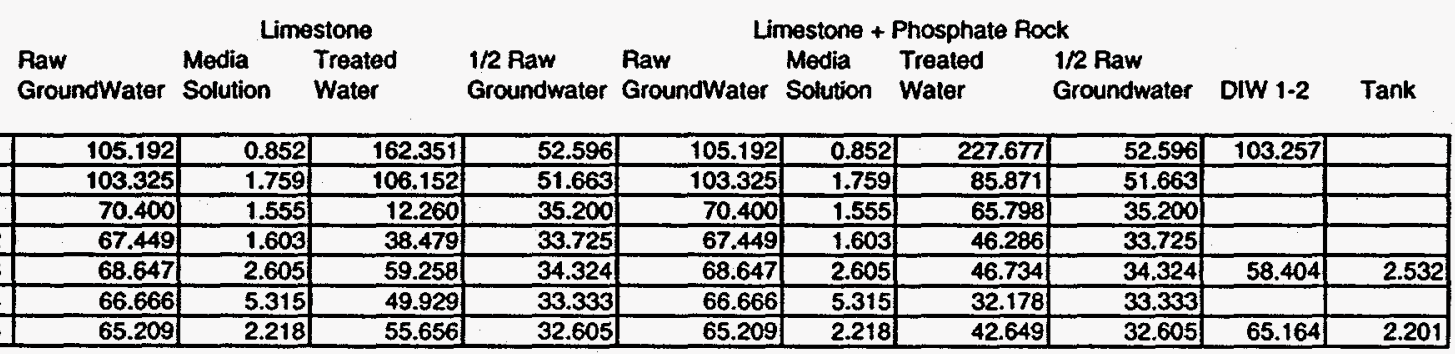

Chromium Analytical Results

\begin{tabular}{|c|c|c|c|c|c|c|c|c|}
\hline \multicolumn{3}{|c|}{ Limestone } & \multicolumn{6}{|c|}{ Limestone + Phosphate Rock } \\
\hline $\begin{array}{l}\text { Raw } \\
\text { GroundWater }\end{array}$ & $\begin{array}{l}\text { Media } \\
\text { Solution }\end{array}$ & $\begin{array}{l}\text { Treated } \\
\text { Water }\end{array}$ & $\begin{array}{l}\text { 1/2 Raw } \\
\text { Groundwater }\end{array}$ & $\begin{array}{l}\text { Raw } \\
\text { GroundWater }\end{array}$ & $\begin{array}{l}\text { Media } \\
\text { Solution }\end{array}$ & $\begin{array}{l}\text { Treated } \\
\text { Water }\end{array}$ & $\begin{array}{l}\text { 1/2 Raw } \\
\text { Groundwater }\end{array}$ & DIW 1-2 \\
\hline
\end{tabular}

\begin{tabular}{|c|c|c|c|c|c|c|c|c|c|c|}
\hline Day 1 & $\mathbf{8 . 2 9 9}$ & $<0.006$ & 0.020 & 4.150 & 8.299 & $<0.006$ & $<0.060$ & 4.150 & 12.402 & \\
\hline Day 2 & 10.700 & $<0.060$ & $<0.060$ & 5.350 & 10.700 & $<0.060$ & $<0.060$ & 5.350 & & \\
\hline Day 3 & 0.150 & $<0.060$ & $<0.060$ & 0.075 & 0.150 & $<0.060$ & $<0.060$ & 0.075 & & \\
\hline Woek 2 & 10.894 & $<0.060$ & 0.060 & 5.447 & 10.894 & $<0.060$ & $<0.060$ & 5.447 & & \\
\hline Week 3 & 12.411 & $<0.060$ & 0.209 & 6.206 & 12.411 & $<0.060$ & 0.173 & 6.206 & 9.953 & $<0.060$ \\
\hline Week 4 & 13.993 & $<0.060$ & $<0.060$ & 6.997 & 13.993 & $<0.060$ & 0.106 & 6.997 & & \\
\hline Week 5 & 13.137 & 0.007 & 0.431 & 6.569 & 13.137 & 0.007 & 1.232 & 6.569 & 12.753 & 0.011 \\
\hline
\end{tabular}

Iron Analytical Results

Raw Limestone

GroundWater Solution Water Groundwater GroundWater Solution Water Groundwater DIW 1-2 Tank

\begin{tabular}{|c|c|c|c|c|c|c|c|c|c|c|}
\hline Day 1 & 184.603 & 0.026 & 27.396 & 92.302 & 184.603 & 0.026 & 25.452 & 92.302 & 203.871 & \\
\hline Day 2 & 193.501 & $<0.046$ & 14.883 & 96.751 & 193.501 & $<0.046$ & 25.514 & 96.751 & & \\
\hline Day 3 & 29.284 & $<0.046$ & $<0.046$ & 14.642 & 29.284 & $<0.046$ & 7.401 & 14.642 & & \\
\hline Wook 2 & 92.860 & $<0.046$ & 16.687 & 46.430 & 92.860 & $<0.046$ & 5.681 & 46.430 & & \\
\hline Woek 3 & 87.869 & $<0.046$ & 25.620 & 43.935 & 87.869 & $<0.046$ & 13.326 & 43.935 & 57.294 & $<0.046$ \\
\hline Week 4 & 69.070 & 0.293 & 10.002 & 34.535 & 69.070 & 0.293 & 8.582 & 34.535 & & \\
\hline Week 5 & 78.084 & 0.141 & 18.901 & 39.042 & 78.084 & 0.141 & 24.346 & 39.042 & 79.247 & 0.147 \\
\hline
\end{tabular}


Magnesium Analytical Results

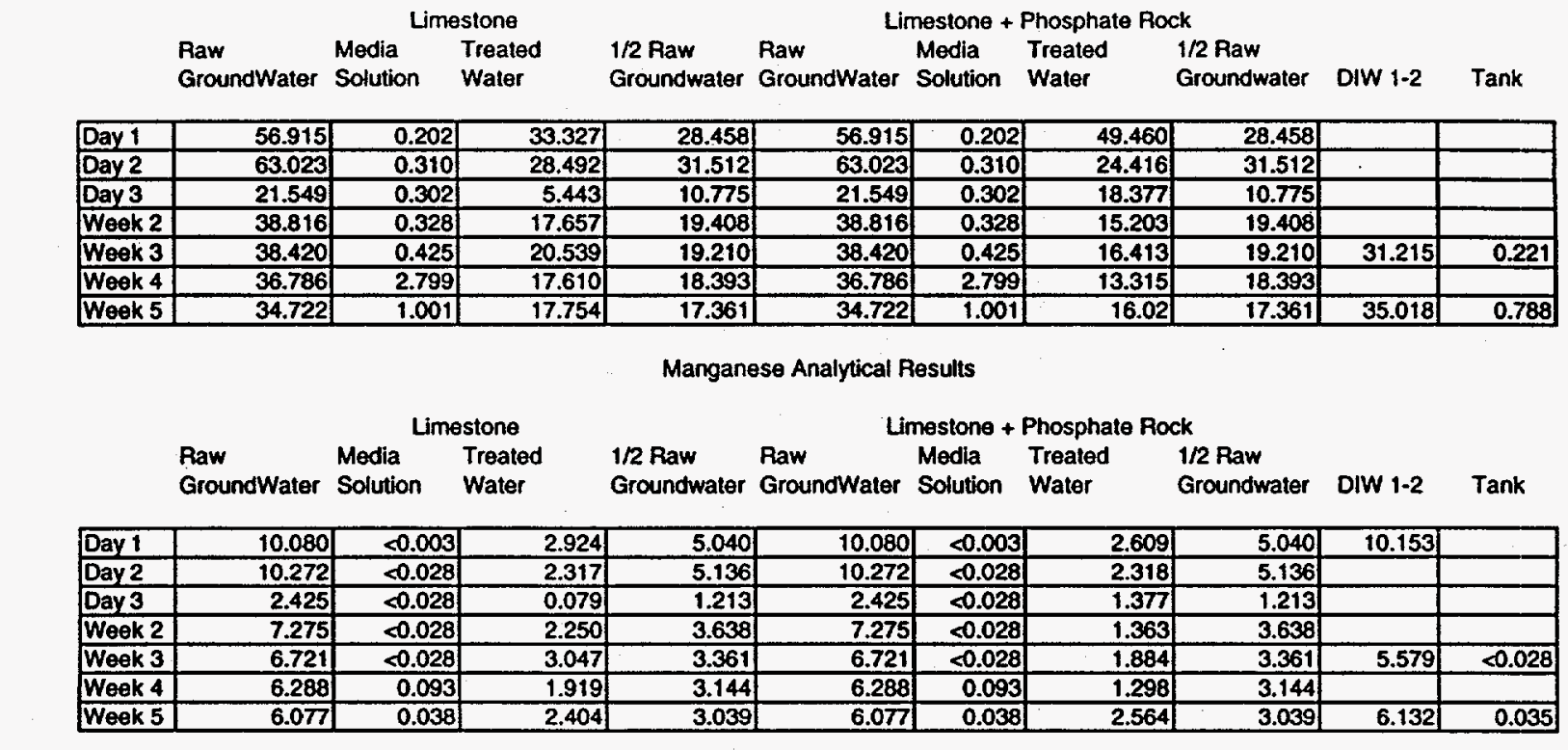

Nickel Analytical Results

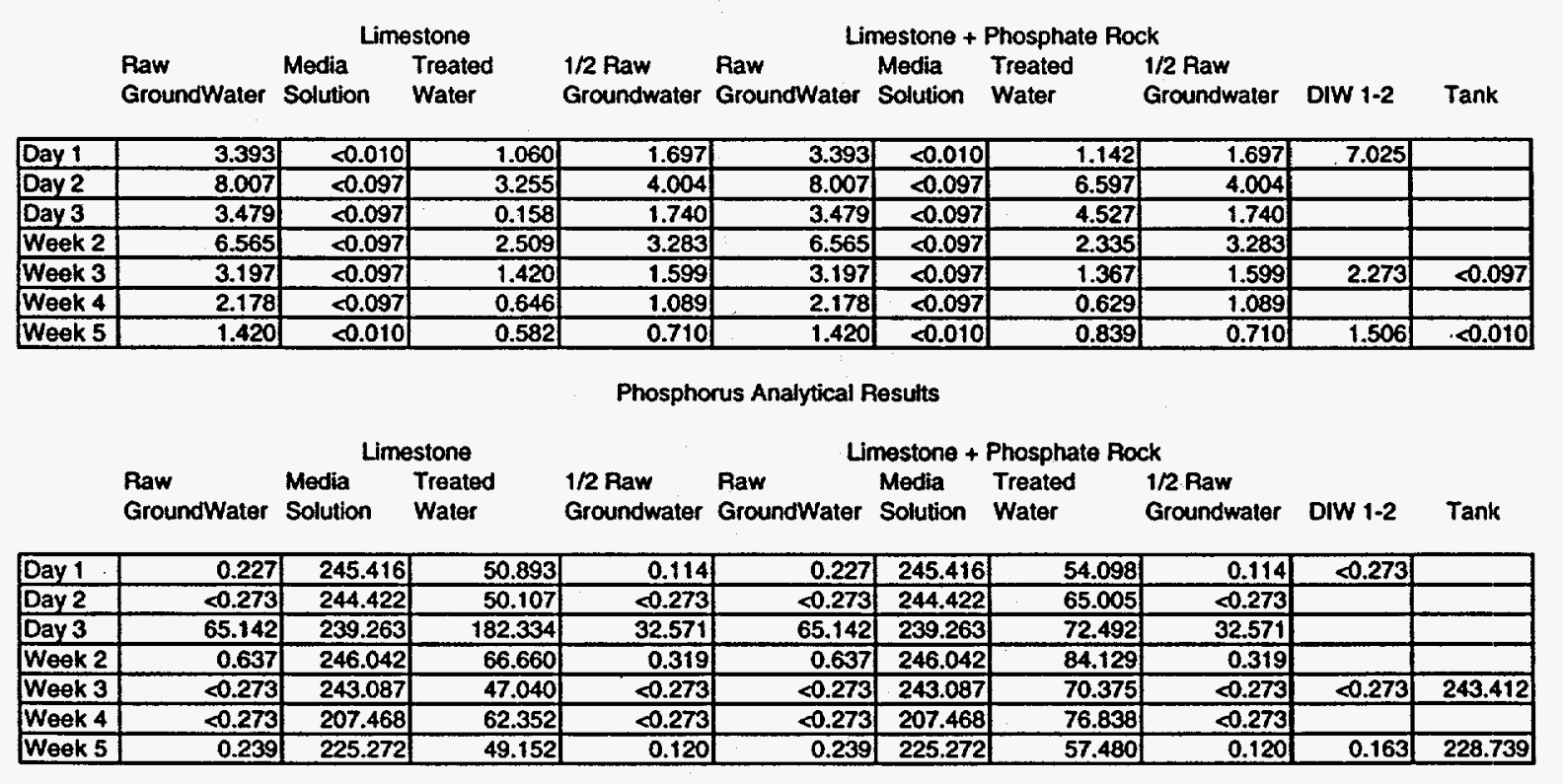


Silica Anatytical Results

\begin{tabular}{lllllll}
\multicolumn{2}{c}{ Limestone } & \multicolumn{4}{c}{ Limestone + Phosphate Rock } \\
Raw & Media & Treated & 1/2 Raw Raw & Media Treated & 1/2 Raw \\
GroundWater Solution & Water & Groundwater GroundWater Solution Water & Groundwater DIW 1-2 Tank
\end{tabular}

\begin{tabular}{|c|c|c|c|c|c|c|c|c|c|c|}
\hline Day 1 & 71.002 & 4.359 & 33.708 & 35.501 & 71.002 & 4.359 & 35.323 & 35.501 & 80.727 & \\
\hline Day 2 & 79.876 & 3.764 & 36.227 & 39.938 & 79.876 & 3.764 & 33.509 & 39.938 & & \\
\hline Day 3 & 30.508 & 3.707 & 10.721 & 15.254 & 30.508 & 3.707 & 29.894 & 15.254 & & \\
\hline Week 2 & 76.637 & 3.952 & 35.367 & 38.319 & 76.637 & 3.952 & 33.714 & 38.319 & & \\
\hline Week 3 & 81.234 & 4.378 & 42.080 & 40.617 & 81.234 & 4.378 & 38.851 & 40.617 & 74.726 & 3.766 \\
\hline Weok 4 & 82.294 & 10.945 & 39.016 & 41.147 & 82.294 & 10.945 & 33.742 & 41.147 & & \\
\hline Weok 5 & 78.911 & 5.974 & 39.874 & 39.4555 & 78.911 & 5.974 & 40.116 & 39.4555 & 78.497 & 5.43 \\
\hline
\end{tabular}

Sulfate Analytical Results

Limestone Limestone + Phosphate Rock

$\begin{array}{lllllll} & \text { Media } & \text { Treated } & \text { I/2 Raw } & \text { Raw } & \text { Media Treated } & \text { I/2 Raw } \\ \text { GroundWater Solution } & \text { Water } & \text { Groundwater GroundWater Solution Water } & \text { Groundwater DIW 1-2 Tank }\end{array}$

\begin{tabular}{|c|c|c|c|c|c|c|c|c|c|c|}
\hline Day 1 & 2322.502 & 293.939 & 1015.949 & 1161.251 & 2322.502 & 293.939 & 1066.359 & 1161.251 & 1924.835 & \\
\hline Day 2 & 2503.553 & 1256.959 & 946.068 & 1251.777 & 2503.553 & 1256.959 & 727.472 & 1251.777 & & \\
\hline Day 3 & 1153.072 & 31.340 & 203.653 & 576.536 & 1153.072 & 31.340 & 584.710 & 576.536 & & \\
\hline Week 2 & 1179.939 & 533.298 & 728.699 & 589.970 & 1179.939 & 533.298 & 473.115 & 589.970 & & \\
\hline Week 3 & 1367.021 & $<10.000$ & 888.541 & 683.511 & 1367.021 & $<10.000$ & 487.755 & 683.511 & 1211.604 & 27.080 \\
\hline Week 4 & 1281.514 & $<10.000$ & 749.688 & 640.757 & 1281.514 & $<10.000$ & 401.357 & 640.757 & & \\
\hline Week 5 & & & & & & & & & & \\
\hline
\end{tabular}

pH Field Results

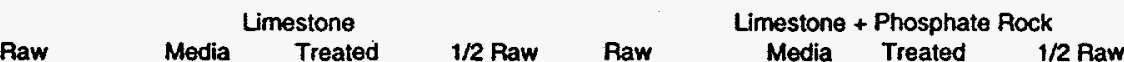

GroundWater Solution Water Groundwater GroundWater Solution Water Groundwater

\begin{tabular}{|l|l|l|l|l|l|l|l|l|}
\hline Day 1 & 2.79 & 8.69 & 5.64 & & 2.79 & 8.69 & 5.86 & \\
\hline Day 2 & 2.73 & 8.61 & 5.54 & & 2.73 & 8.61 & 5.65 & \\
\hline Day 3 & 5.77 & 8.57 & 7.16 & & 5.77 & 8.57 & 6.10 & \\
\hline Week 2 & 3.51 & 5.27 & 5.65 & & 3.51 & 5.27 & 6.32 & \\
\hline Week 3 & 3.32 & 8.17 & 5.23 & & 3.32 & 8.17 & 4.73 & \\
\hline Week 4 & 3.39 & 8.45 & 5.63 & & 3.39 & 8.45 & 6.06 & \\
\hline Week 5 & 3.65 & 8.34 & 5.96 & & 3.65 & 8.34 & 6.20 & \\
\hline
\end{tabular}

mV Fioid Results

Limestone Limestone + Phosphate Rock

Raw Media Treated 1/2 Raw Raw Media Treated 1/2 Raw

GroundWater Solution Water Groundwater GroundWater Solution Water Groundwater

\begin{tabular}{|c|c|c|c|c|c|c|}
\hline Day 1 & 185.1 & 19.5 & -88.8 & 185.1 & 19.5 & -130.6 \\
\hline Day 2 & 137.0 & -77.0 & -70.9 & 137.0 & -77.0 & -115.4 \\
\hline Day 3 & -48.4 & 12.8 & -123.6 & -48.4 & 12.8 & -73.4 \\
\hline Woek 2 & 115.6 & $\$ 18.6$ & 2.3 & 115.6 & 118.6 & -60.8 \\
\hline Week 3 & 122.4 & 28.8 & 34.5 & 122.4 & 28.8 & 48.6 \\
\hline Week 4 & 123.1 & 108.8 & 25.8 & 123.1 & 108.8 & -15.4 \\
\hline Week 5 & 119.8 & 153.9 & 8.2 & 119.8 & 153.9 & -16.2 \\
\hline
\end{tabular}


En Field Results $(\mathrm{mV}+197 \mathrm{mV})$

\begin{tabular}{|c|c|c|c|c|c|c|c|}
\hline \multicolumn{4}{|c|}{ Limestone } & \multicolumn{4}{|c|}{ Limestone + Phosphate Rock } \\
\hline $\begin{array}{l}\text { Raw } \\
\text { GroundWater }\end{array}$ & $\begin{array}{l}\text { Media } \\
\text { Solution }\end{array}$ & $\begin{array}{l}\text { Treated } \\
\text { Water }\end{array}$ & $\begin{array}{l}\text { 1/2 Raw } \\
\text { Groundwater }\end{array}$ & $\begin{array}{l}\text { Raw } \\
\text { GroundWater }\end{array}$ & $\begin{array}{l}\text { Media } \\
\text { Solution }\end{array}$ & $\begin{array}{l}\text { Treated } \\
\text { Water }\end{array}$ & $\begin{array}{l}\text { 1/2 Raw } \\
\text { Groundwater }\end{array}$ \\
\hline 382.1 & 216.5 & 108.2 & & 382.1 & 216.5 & 66.4 & \\
\hline 334.0 & 120.0 & 126.1 & & 334.0 & 120.0 & 81.6 & \\
\hline 148.6 & 209.8 & 73.4 & & 148.6 & 209.8 & 123.6 & \\
\hline 312.6 & 315.6 & 199.3 & & 312.6 & 315.6 & 136.2 & \\
\hline 319.4 & 225.8 & 231.5 & & 319.4 & 225.8 & 245.6 & \\
\hline 320.1 & 305.8 & 222.8 & & 320.1 & 305.8 & 181.6 & . \\
\hline 316.8 & 350.9 & 205.2 & & 316.8 & 350.9 & 180.8 & \\
\hline
\end{tabular}

Phosphate Analytical Results

\begin{tabular}{lllllll}
\multicolumn{2}{c}{ Limestone } & \multicolumn{4}{c}{ Limestone + Phosphate Rock } \\
Raw & Media & Treated & 1/2 Raw & Raw & Media Treated & 1/2 Raw \\
GroundWater Solution & Water & Groundwater GroundWater Solution Water & Groundwater DIW 1-2 Tank
\end{tabular}

\begin{tabular}{|c|c|c|c|c|c|c|c|c|c|c|}
\hline Day 1 & 110.516 & 754.566 & 199.113 & 55.258 & 110.516 & 754.566 & 226.798 & 55.258 & 11.455 & \\
\hline Day 2 & 53.980 & 217.975 & $\$ 89.053$ & 26.990 & 53.980 & 217.975 & 256.825 & 26.990 & & \\
\hline Day 3 & 202.073 & 834.343 & 577.557 & 101.037 & 202.073 & 834.343 & 298.303 & 101.037 & & \\
\hline Week 2 & 124.477 & 389.204 & 225.252 & 62.239 & 124.477 & 389.204 & 271.196 & 62.239 & & \\
\hline Week 3 & 124.053 & 677.955 & 166.802 & 62.027 & 124.053 & 677.955 & 210.222 & 62.027 & 113.427 & 781.106 \\
\hline Week 4 & 122.812 & 717.152 & 256.264 & 61.406 & 122.812 & 717.152 & 243.739 & 61.406 & & \\
\hline Week 5 & & & & & & & & & & \\
\hline
\end{tabular}




\section{PRELIMINARY LABORATORY}




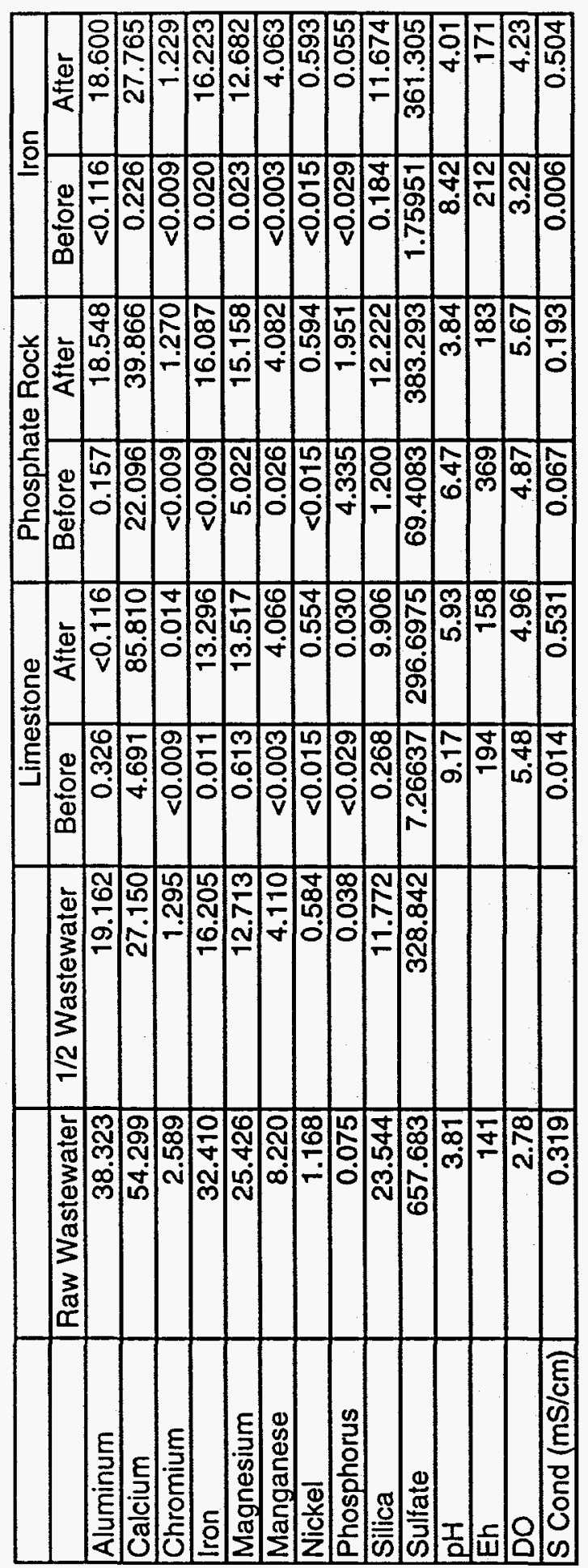




\section{LAB TEST \#1}




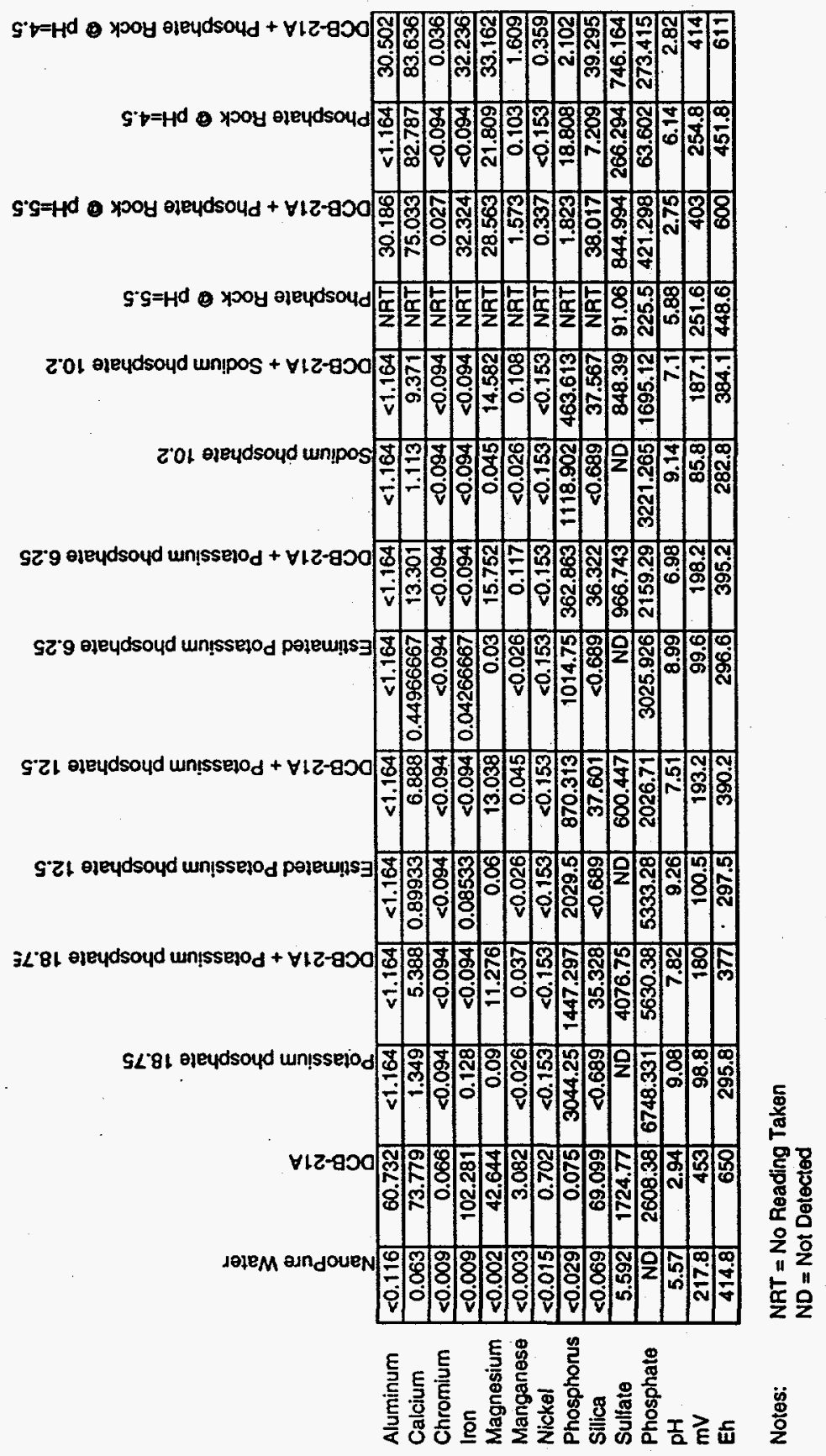




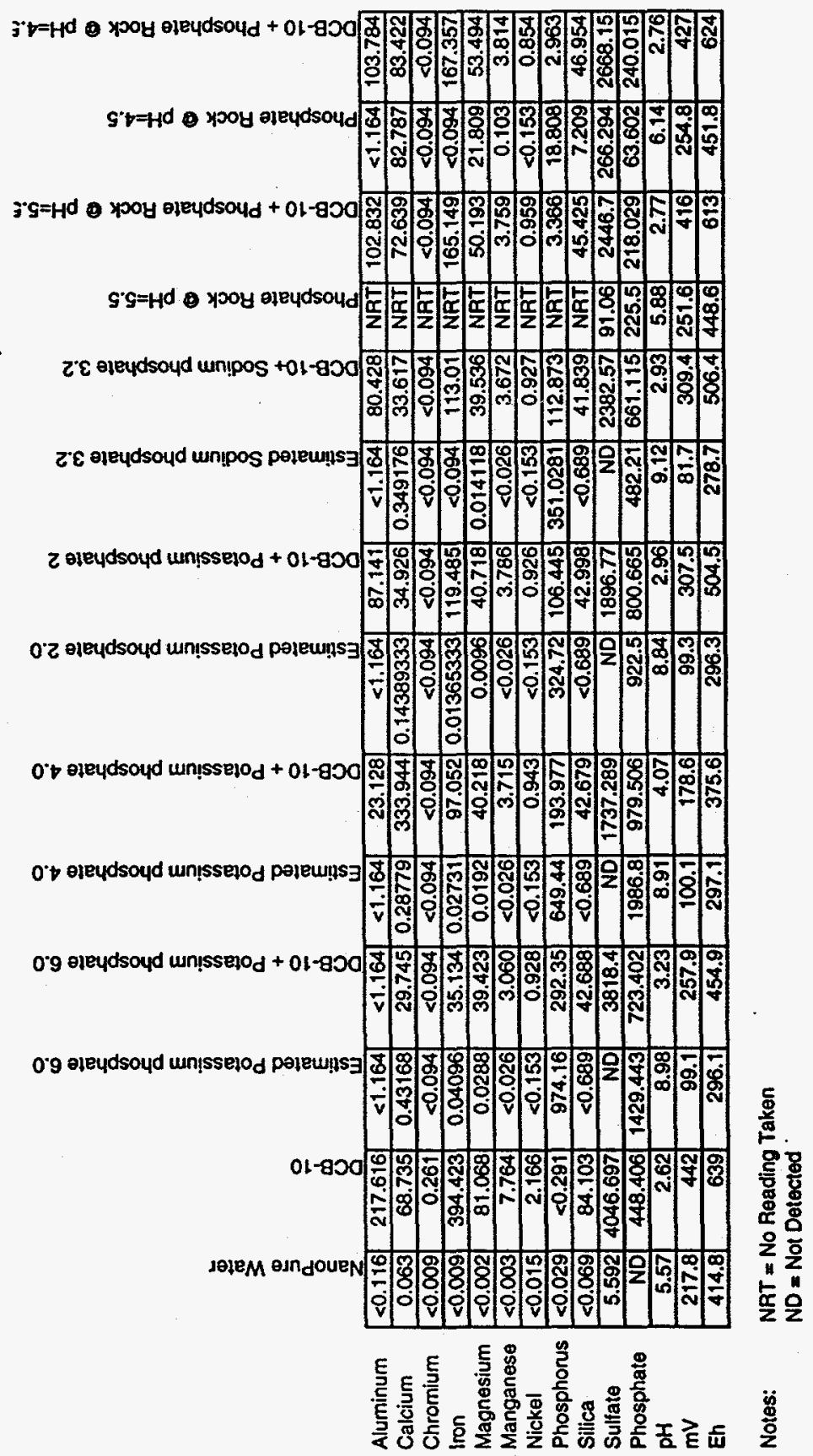




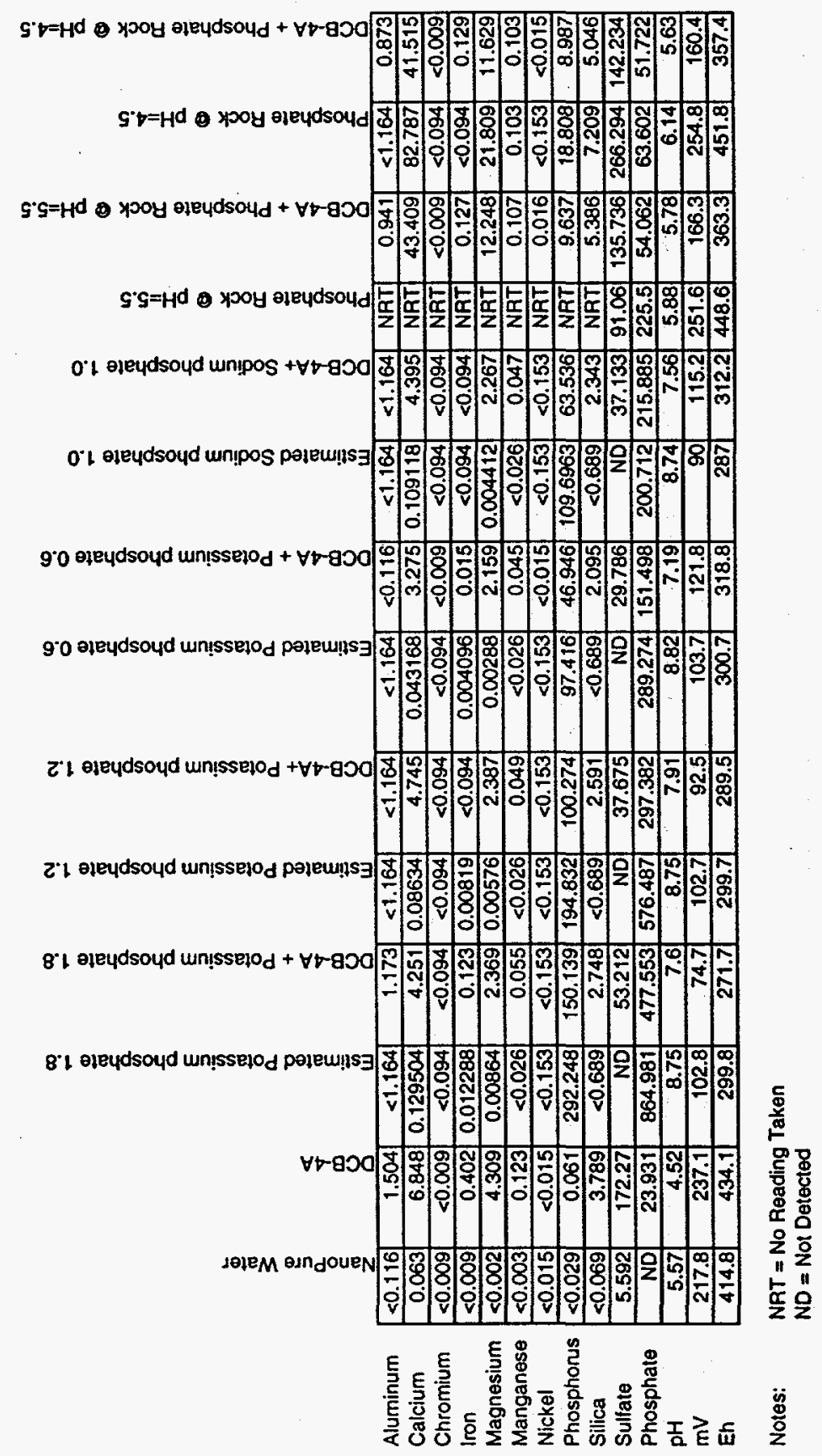


LAB TEST \#2 


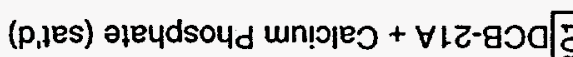

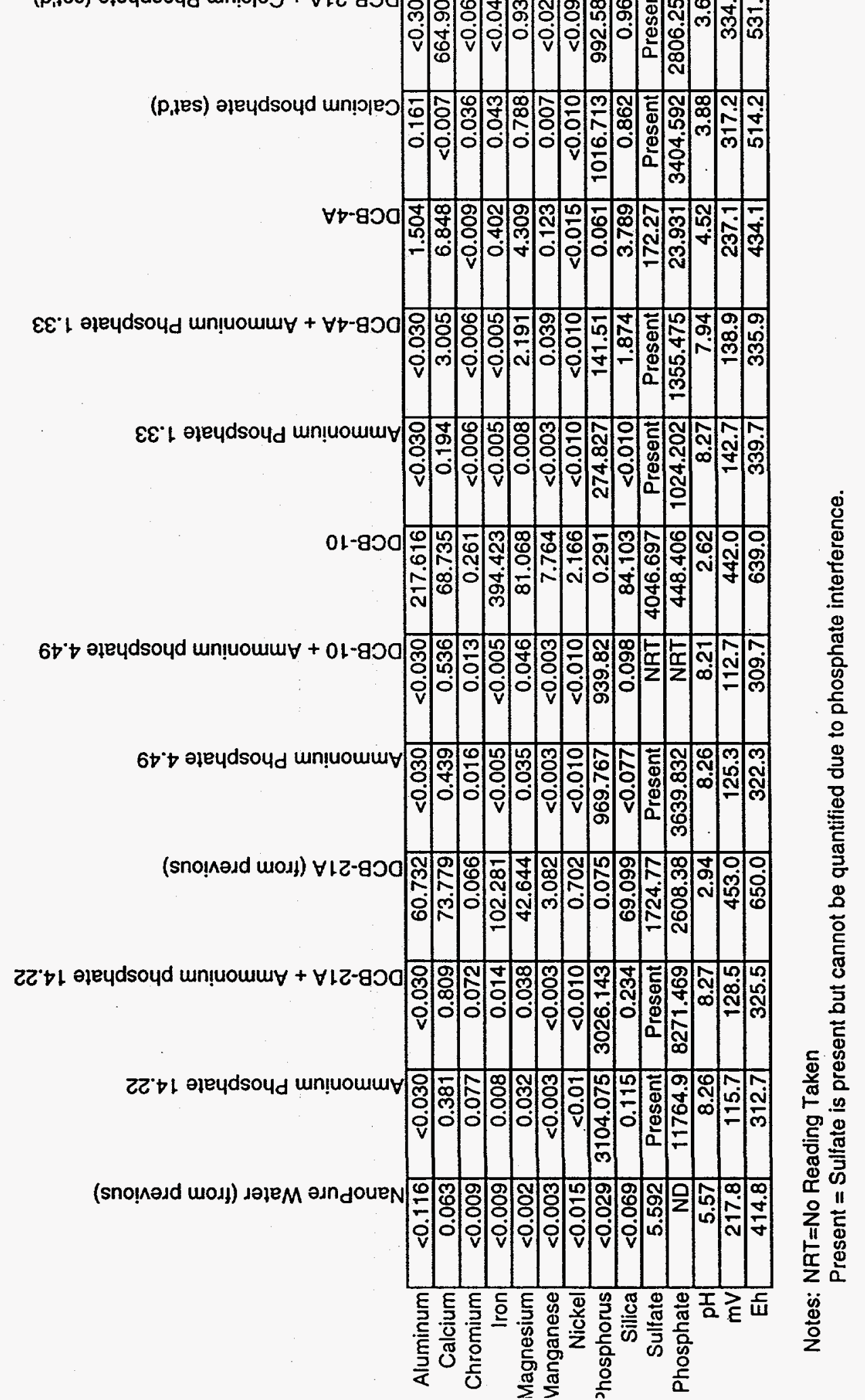




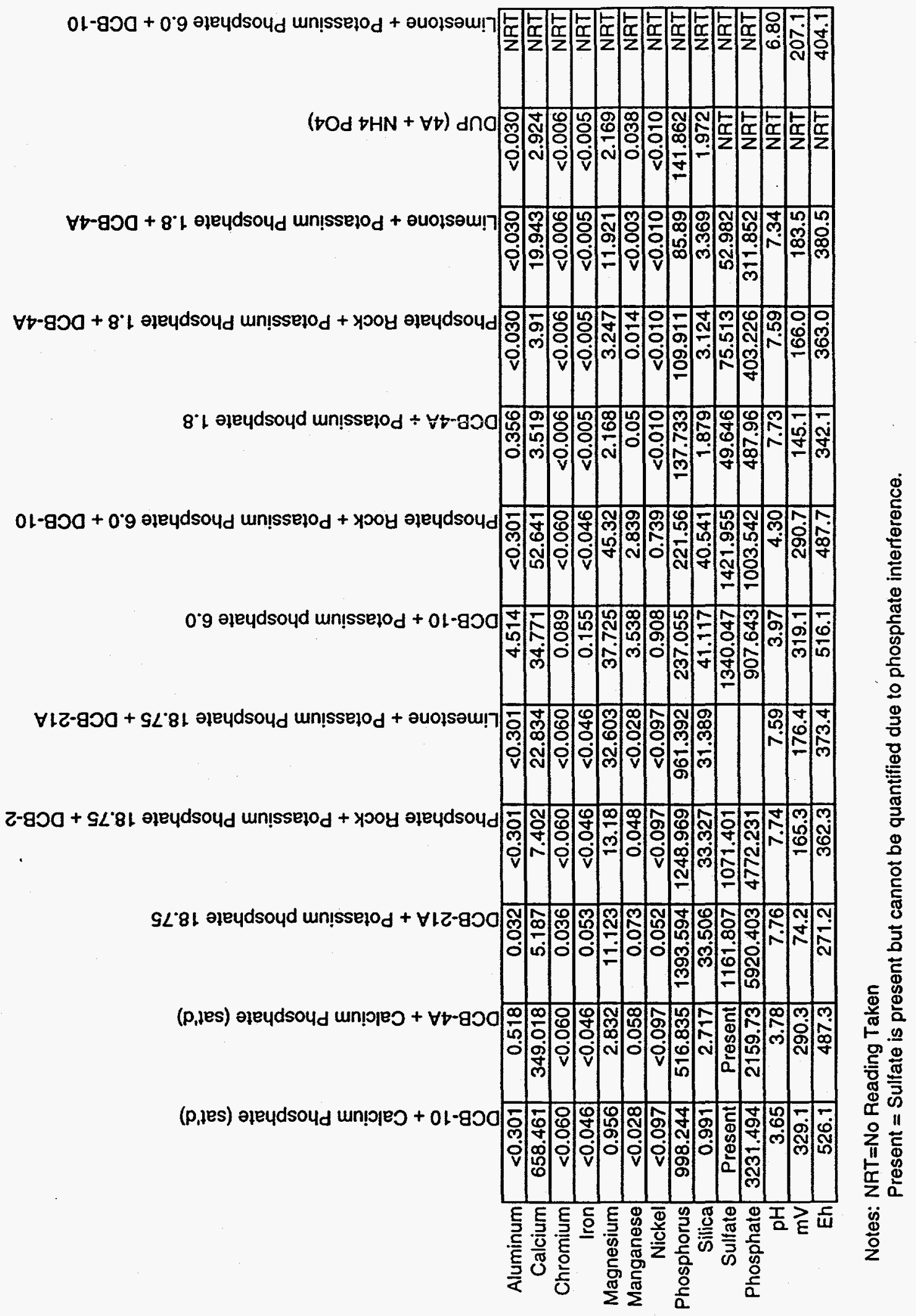


LAB TEST 3 \& 4 


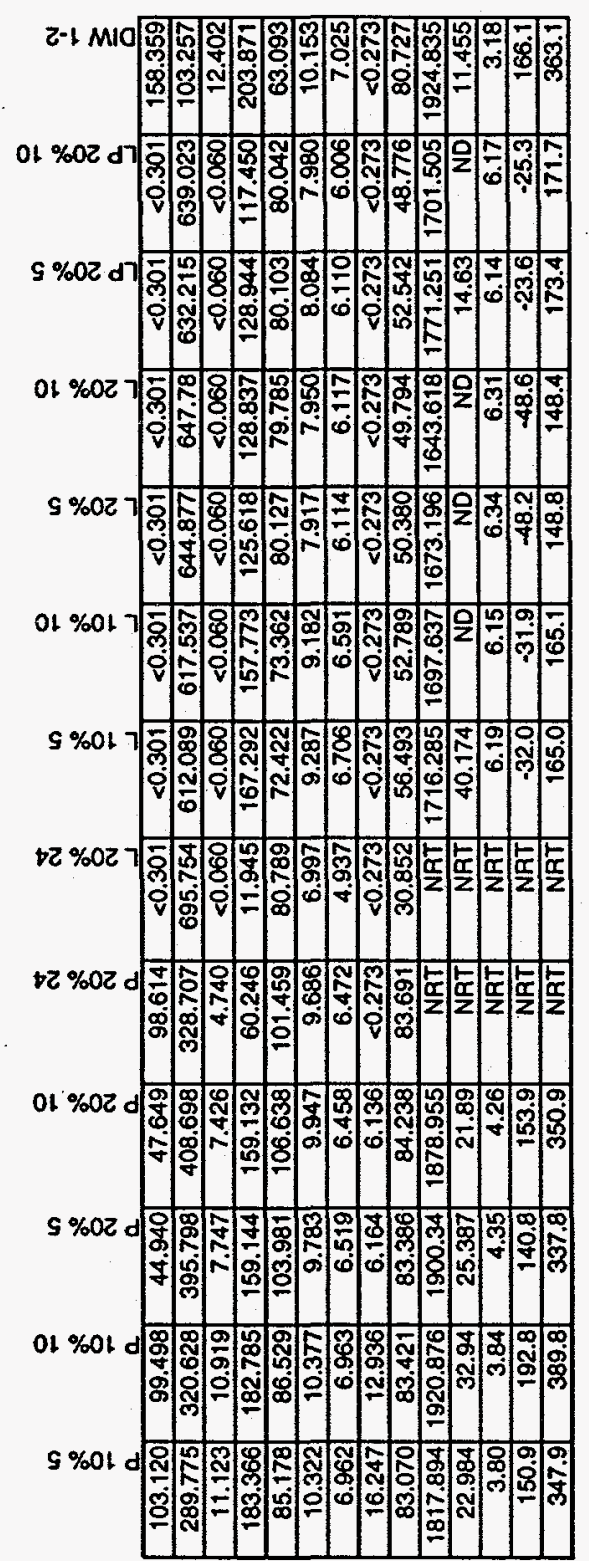

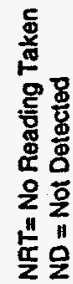

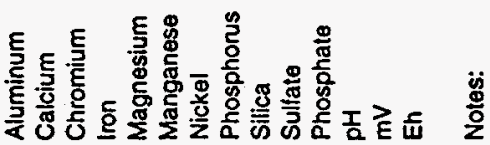
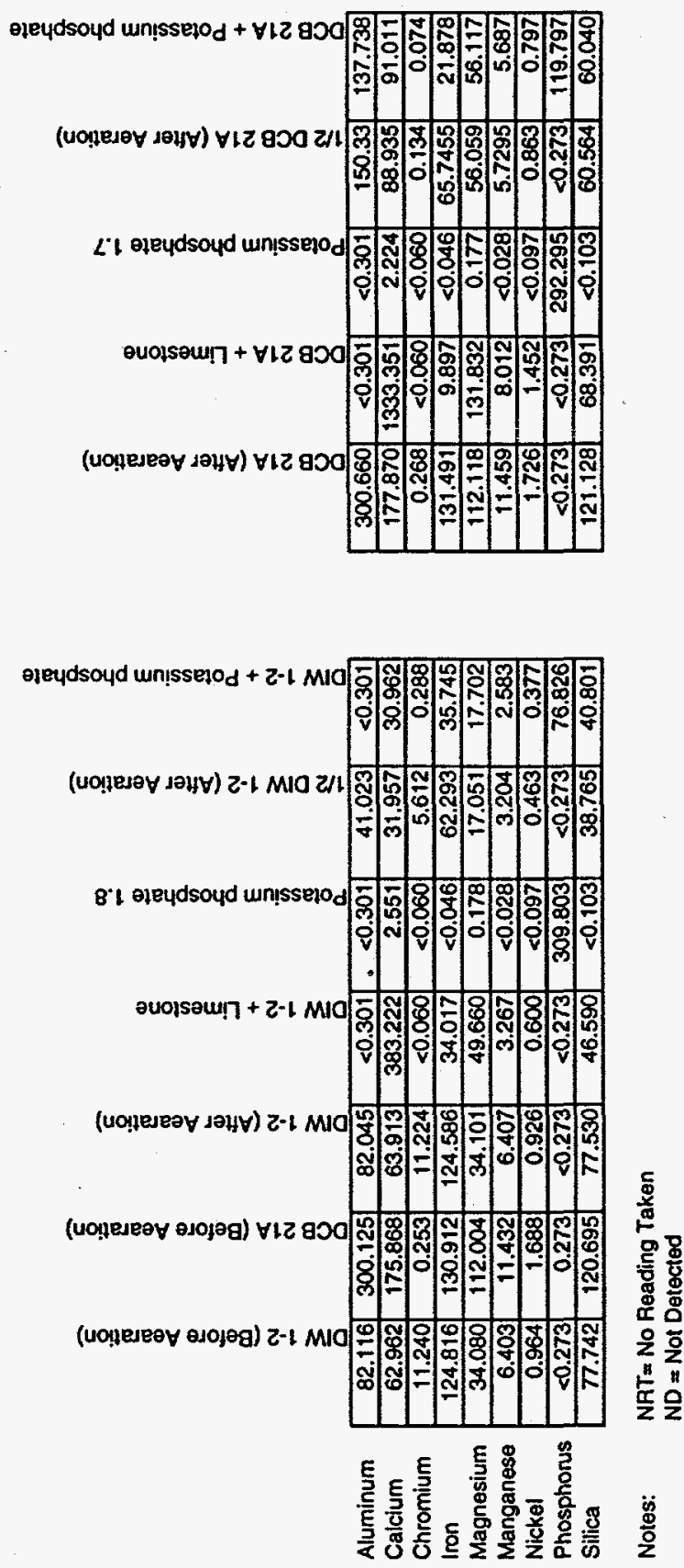


\section{PRESSURE MEASUREMENTS}




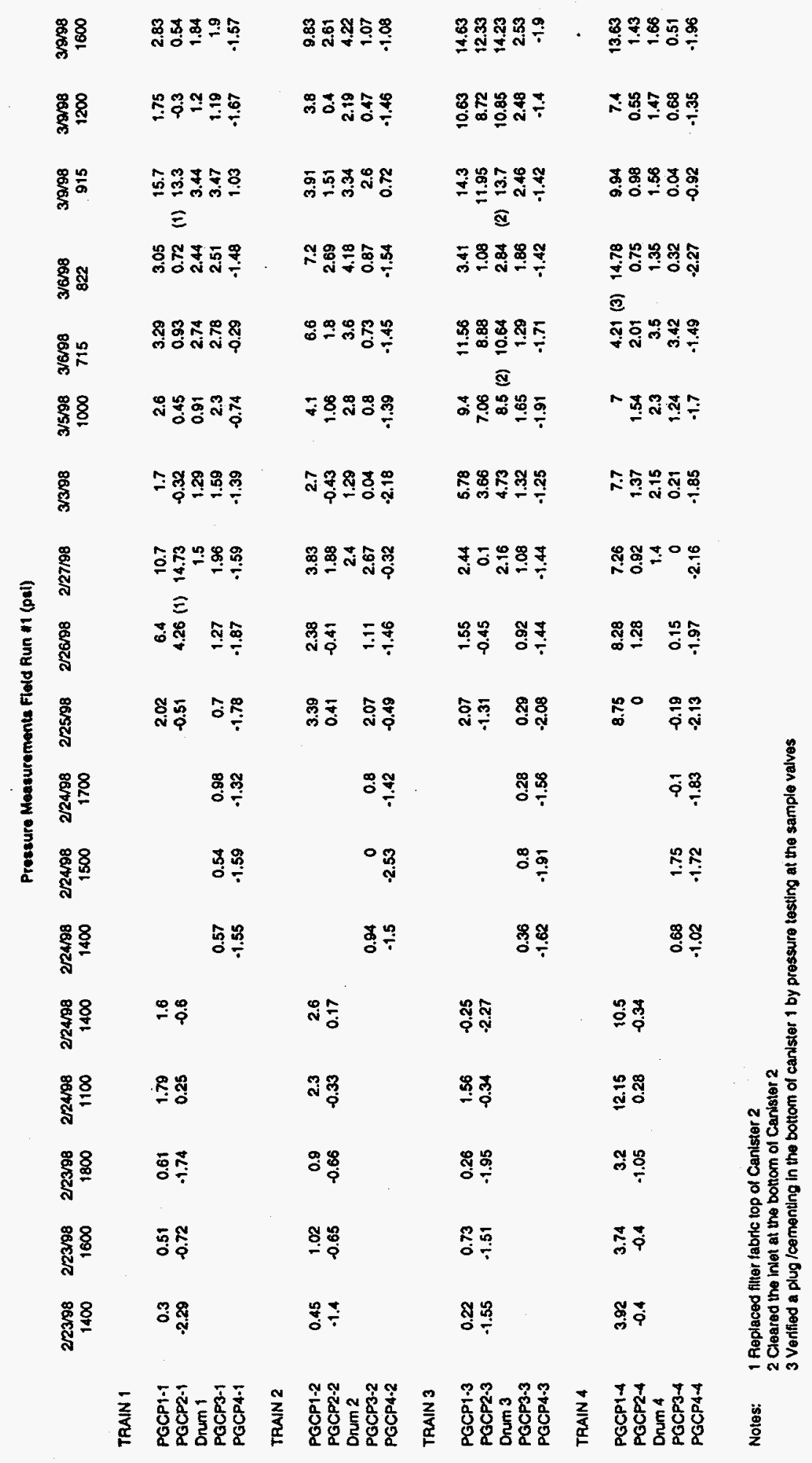




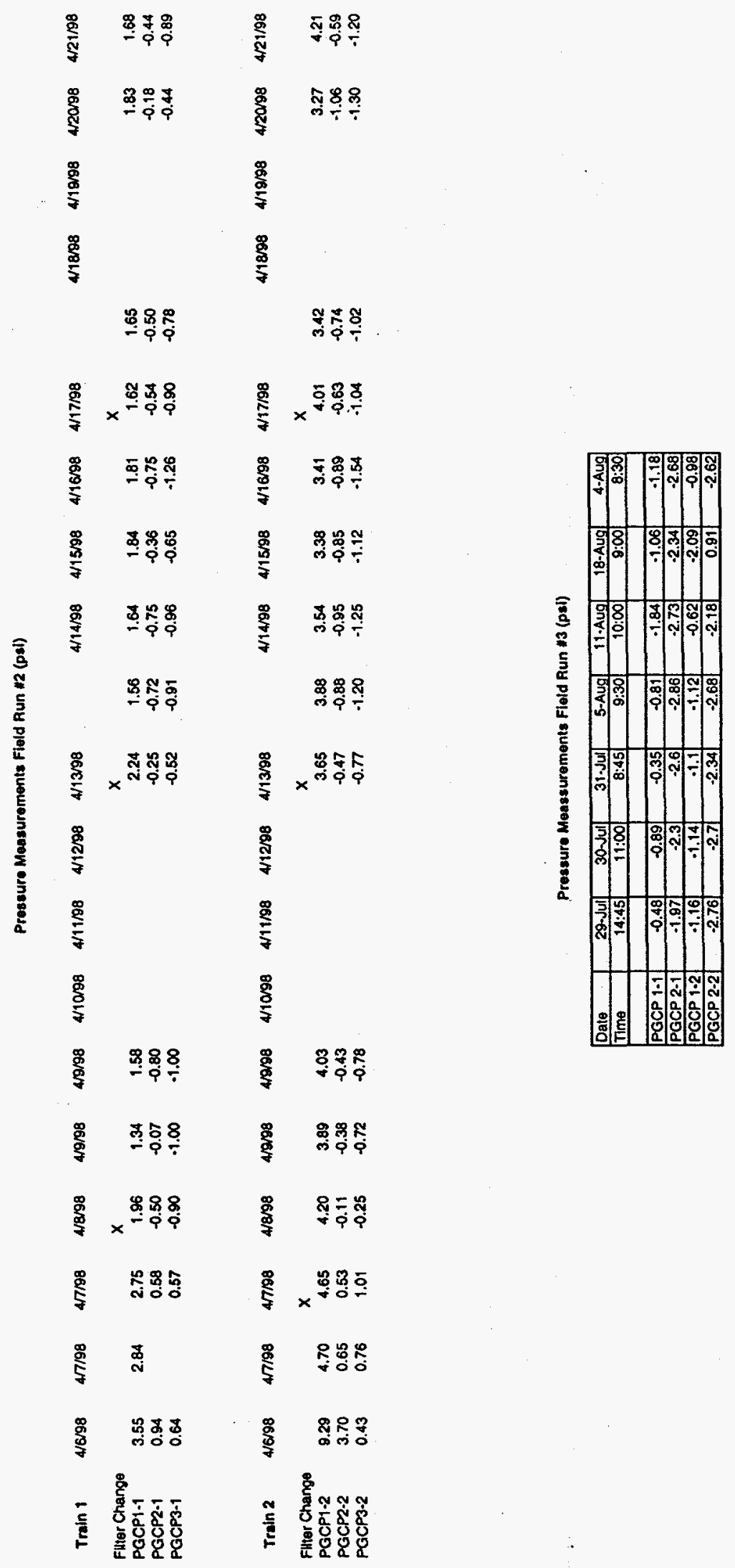

